\title{
Supporting Information for: Theoretical Investigations of (Oxidative) Dehydrogenation of Propane to Propylene Over Palladium Surfaces
}

\author{
Eduard Araujo-Lopez, ${ }^{\dagger}$ Lennart Joos, ${ }^{\S}$ Bart D. Vandegehuchte, ${ }^{\S}$ Dmitry I. Sharapa, ${ }^{\dagger}$ and Felix Studt ${ }^{*}, \dagger$, \\ ${ }^{\dagger}$ Institute of Catalysis Research and Technology, Karlsruhe Institute of Technology, Hermann-von-Helmholtz Platz 1, 76344 \\ Eggenstein-Leopoldshafen, Germany \\ $\S$ Total Research \& Technology Feluy, Zone Industrielle Feluy C, B-7181, Seneffe, Belgium \\ $\$$ Institute for Chemical Technology and Polymer Chemistry, Karlsruhe Institute of Technology, Engesserstrasse 18, 76131 \\ Karlsruhe, Germany
}

\section{Adsorptions over Pd(111) and Pd(211) surfaces}

(a) $\operatorname{Pd}(111)$

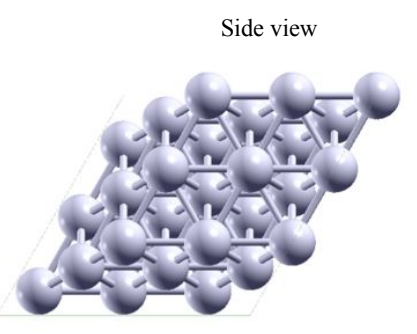

(b) Pd (211)

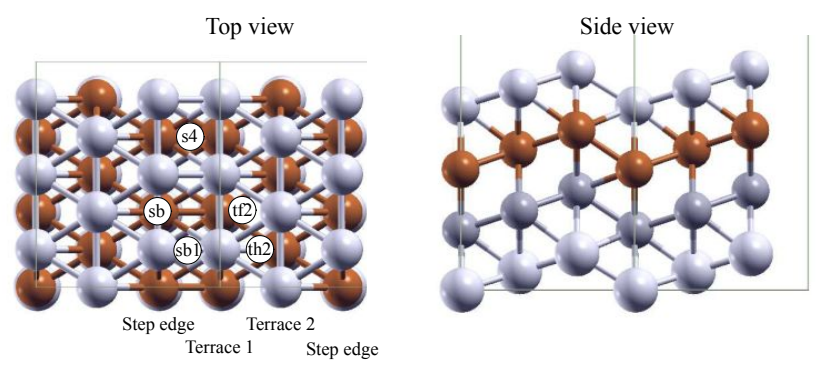

Figure S1. $\operatorname{Pd}(111)$ and $\operatorname{Pd}(211)$ surfaces. Adsorption sites are abbreviated as: tf2, fcc hollow site between terrace 2 and step edge; th2, hcp hollow site between terrace 2 and step edge; sb1, bridge site perpendicular to step edge; $\mathbf{s} \mathbf{4}$, fourfold hollow site in step; $\mathbf{s b}$, bridge site parallel to step edge. Based on Orita et al. ${ }^{1}$

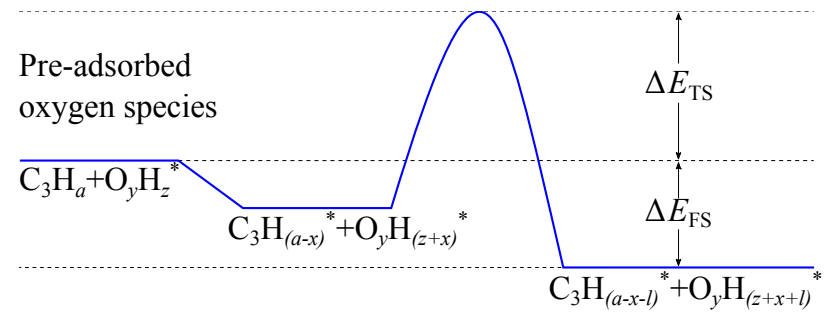

Figure S2. Potential energy diagram for propane dehydrogenation.

\section{Adsorption of $\mathrm{H}, \mathrm{O}, \mathrm{OH}$ species}

In the first part of this study, the adsorptions of hydrogen, oxygen, and hydroxyl species were studied, in order to identify how strong they are, and to prepare the pre-covered oxygen Pd surfaces. The adsorption energies of hydrogen over the $\operatorname{Pd}(111)$ and (211) surfaces are presented in Table S1. On the $\operatorname{Pd}(111)$ surface, atomic hydrogen prefers to adsorb on the fcc site; the most stable adsorptions over $\operatorname{Pd}(211)$ is the tf 2 site. These values are in good agreement with previous theoretical studies. ${ }^{2,3}$

Table S1. Adsorption energies of hydrogen over Pd surfaces

\begin{tabular}{llll}
\hline Site & $\operatorname{Pd}(111)$ & Site & $\operatorname{Pd}(211)$ \\
& $E_{a d s}[\mathrm{eV}]$ & & $E_{a d s}[\mathrm{eV}]$ \\
\hline fcc & -0.35 & tf2 & -0.28 \\
hcp & -0.31 & th2 & -0.27 \\
atop & +0.12 & sb1 & -0.16 \\
\hline
\end{tabular}

The adsorption energies of atomic oxygen and hydroxyl on $\operatorname{Pd}(111)$ are investigated and compared with those on $\operatorname{Pd}(211)$, the results are listed on Table S2. The adsorption of atomic oxygen is preferred on fcc sites over both surfaces, fcc on $\operatorname{Pd}(111)$ and tf 2 on $\operatorname{Pd}(211)$, respectively. In the case of hydroxyl, the bridges of the surfaces are preferred as adsorption sites. There is a good agreement between the results obtained here and the values reported previously. ${ }^{4-6}$

Table S2. Adsorption energies of atomic oxygen and hydroxyl

\begin{tabular}{lllllll}
\hline Site & \multicolumn{2}{c}{$\mathrm{Pd}(111) E_{\text {ads }}[\mathrm{eV}]$} & Site & & \multicolumn{2}{l}{$\mathrm{Pd}(211) E_{\text {ads }}[\mathrm{eV}]$} \\
\cline { 2 - 3 } \cline { 6 - 7 } & $\mathrm{O}$ & $\mathrm{OH}$ & & $\mathrm{O}$ & $\mathrm{OH}$ \\
\hline fcc & -1.75 & -2.25 & $\mathrm{tf} 2$ & -1.73 & -2.29 \\
hcp & -1.58 & -2.09 & $\mathrm{sb} / \mathrm{sb} 1$ & -1.55 & $-2.70 /-2.67$ \\
atop & -0.24 & - & $\mathrm{s} 4$ & -1.37 & - \\
bridge & - & -2.28 & $\mathrm{th} 2$ & -1.64 & - \\
\hline
\end{tabular}




\section{C3 species}

The possible adsorption configurations of propane, 1- and 2-propyl, and propylene were optimized by varying the location of the adsorbates over both surfaces, the adsorption energies are presented in Figure S2. The low $E_{a d s}$ for propane indicates a physisorption with an undistorted structure in both surfaces, in the case of the $\operatorname{Pd}(111)$ the adsorption energy is in good agreement with the experimental value of $-0.46 \mathrm{eV} .{ }^{7}$ For the propyl radicals 1 ,- and 2propyl, the $E_{a d s}$ over the $\operatorname{Pd}(211)$ are stronger as typical for chemisorbed species. The adsorption energies of propylene over $\mathrm{Pd}$ are similar to those over single atom alloys of Pt catalysts. Previous calculations done with different functionals for various surfaces for the adsorption/desorption of propene have shown that this variable can be used as a good descriptor of the selectivity of the catalysts, the stronger adsorption the less selective is the catalyst. ${ }^{8-12}$

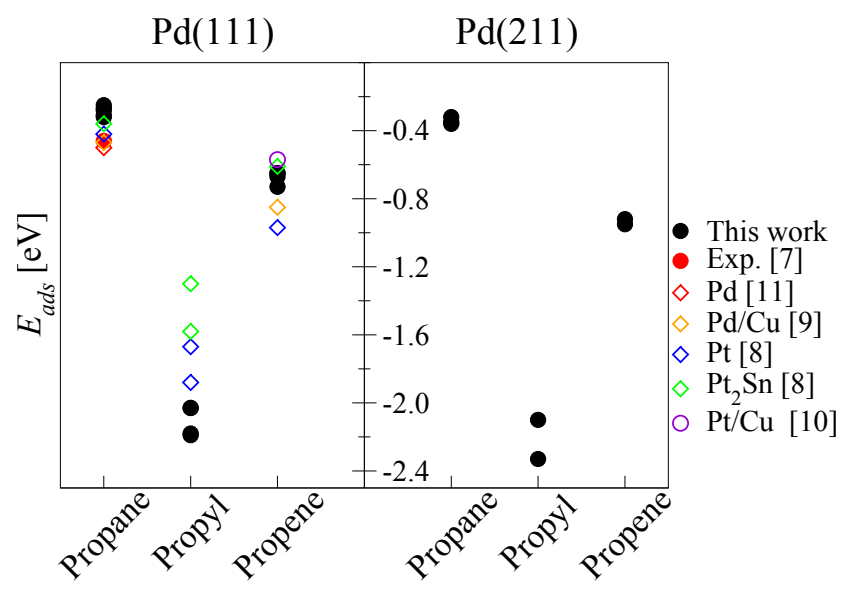

Figure S3. Adsorption energies of C3 species over Pd surfaces and comparison with literature.

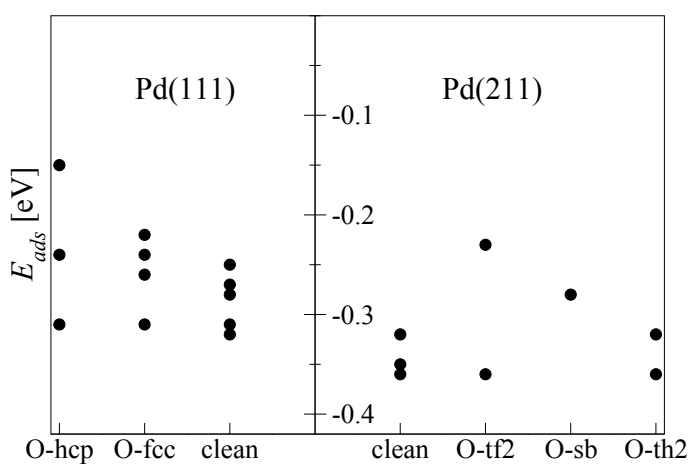

Figure S4. Adsorption energies of propane over O* pre-covered Pd surfaces. Adsorption sites are abbreviated as: tf2, fcc hollow site between terrace 2 and step edge; th2, hcp hollow site between terrace 2 and step edge; $\mathbf{s} 4$, fourfold hollow site in step; $\mathbf{s b}$, bridge site parallel to step edge.

Table S3. Difference in energy [eV] of initial, transition, and final states between $(3 \times 3) /(3 \times 3)$ and $(4 \times 4) /(6 \times 6)$ supercells of $\mathrm{Pd}(111) / \mathrm{Pd}(211)$ for the direct and $\mathrm{OH}$-assisted first dehydrogenation steps.

\begin{tabular}{lllll}
\hline & & Propane & TS1 & 1-Propyl \\
\hline \multirow{2}{*}{$\operatorname{Pd}(111)$} & Direct & 0.02 & 0.03 & 0.02 \\
& OH-assisted & -0.01 & - & -0.04 \\
\hline \multirow{2}{*}{$\operatorname{Pd}(211)$} & Direct & 0.00 & - & 0.00 \\
& OH-assisted & -0.02 & - & 0.00 \\
\hline
\end{tabular}

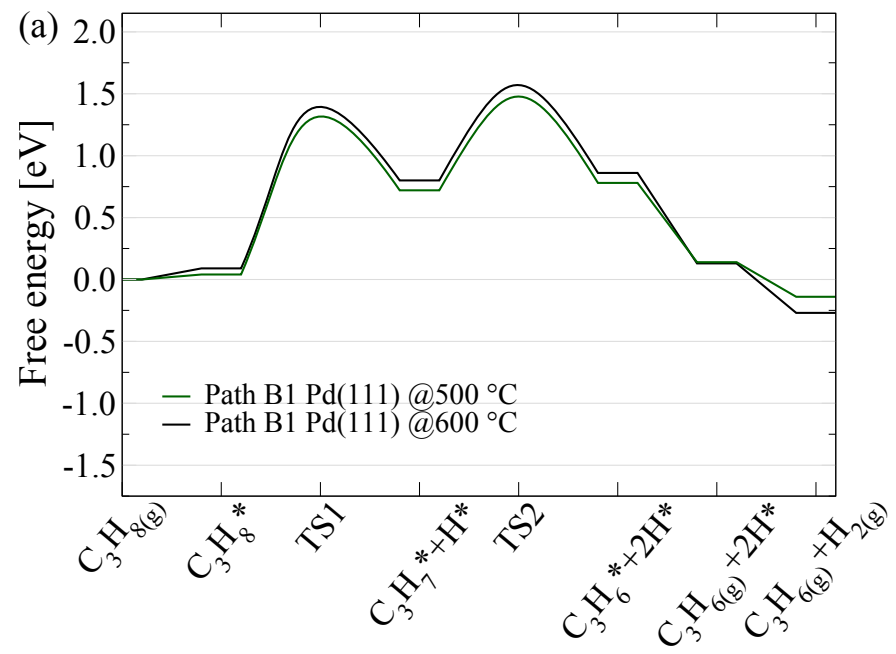

Reaction coordinate

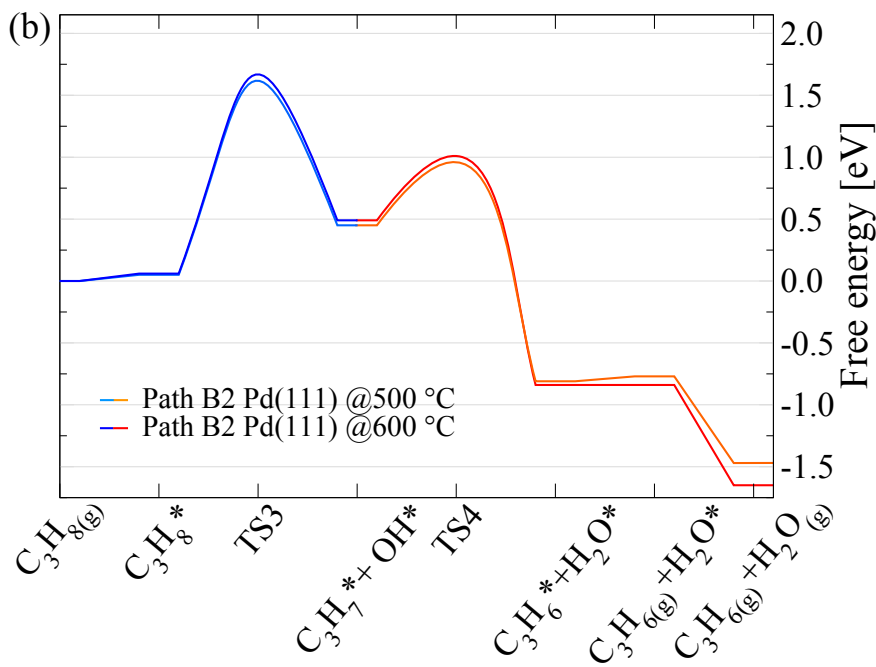

Reaction coordinate

Figure S5. Free energy profiles of (a) DHP and (b) ODHP trough 2-propyl at 500 and $600{ }^{\circ} \mathrm{C}$ 
Table S4. Activation energies of the dehydrogenation of propane over Pd surfaces

\begin{tabular}{|c|c|c|c|c|c|c|c|c|}
\hline \multirow[b]{3}{*}{$E_{a}[\mathrm{eV}]$} & \multicolumn{4}{|l|}{ DHP } & \multicolumn{4}{|l|}{ ODHP } \\
\hline & 111 & & 211 & & 111 & & 211 & \\
\hline & 1-Propyl & 2-Propyl & 1-Propyl & 2-Propyl & 1-Propyl & 2-Propyl & 1-Propyl & 2-Propyl \\
\hline 1st DH & 1.10 & 1.31 & 0.96 & 1.15 & 1.51 & 1.61 & 1.66 & 1.62 \\
\hline 2nd DH & 0.74 & 0.77 & 0.64 & 0.26 & 0.35 & 0.51 & 0.40 & 0.51 \\
\hline
\end{tabular}

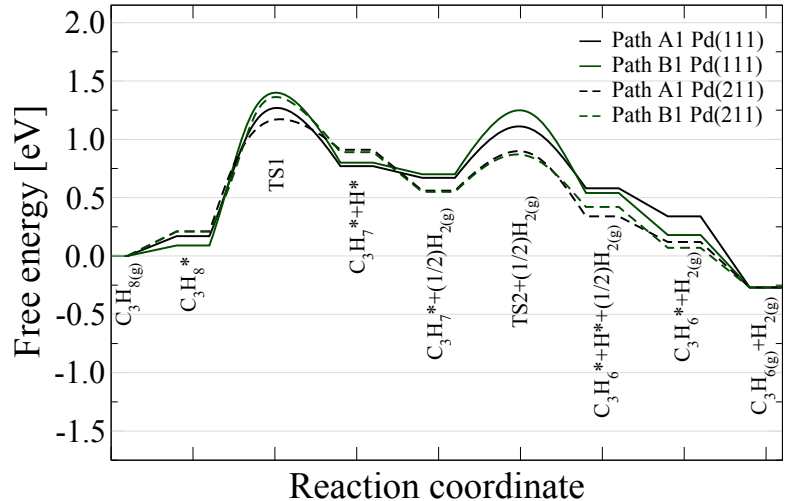

Figure S6. Free energy profile of DHP on Pd surfaces relative to gas-phase $\mathrm{H}_{2}$ at $600{ }^{\circ} \mathrm{C}$.

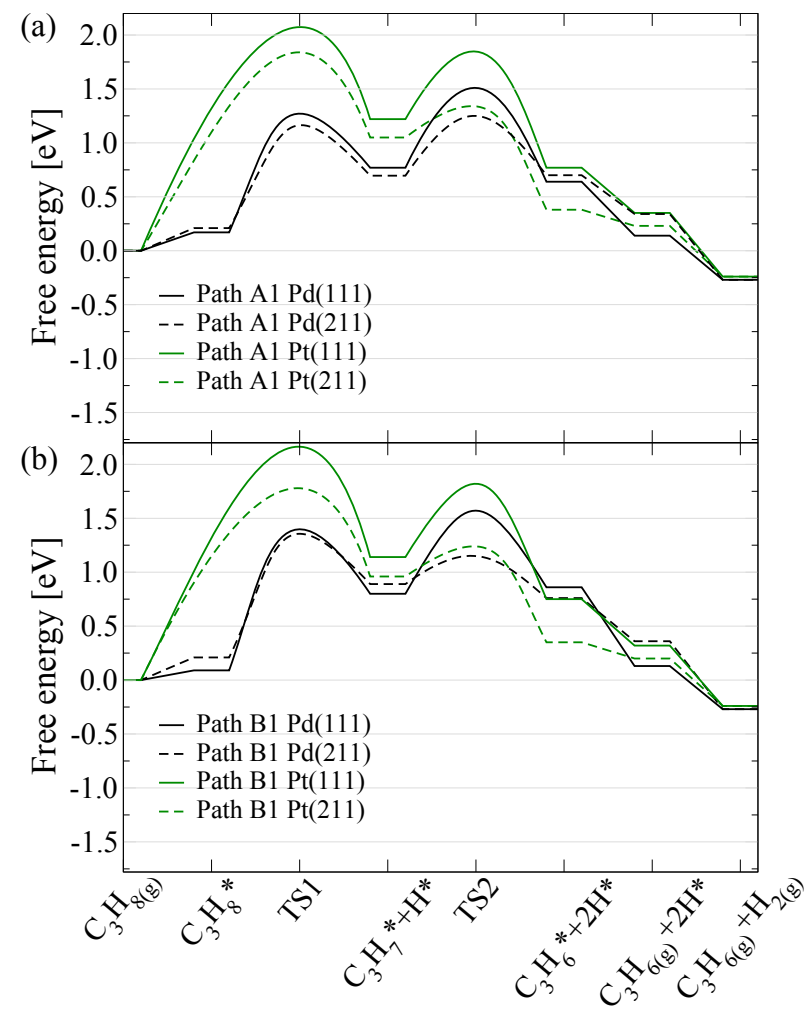

Reaction coordinate

Figure S7. Free energy profile of DHP trough (a) 1-propyl and (b) 2-propyl at $600{ }^{\circ} \mathrm{C}$ on Pd and Pt surfaces. Free energy profiles of the direct DHP taken from Zha et al. ${ }^{12}$
Table S5. D3 energies [eV] (relative to gas phase) of the PBE+D3 single points calculations

\begin{tabular}{llllll}
\hline \multirow{2}{*}{$\operatorname{Pd}(111)$} & & TS1 & Propyl & TS2 & Propene \\
& Path A1 & -0.50 & -0.50 & -0.63 & -0.65 \\
$\operatorname{Pd}(211)$ & Path B1 & -0.61 & -0.61 & -0.63 & -0.67 \\
& Path A1 & -0.46 & -0.48 & -0.54 & -0.59 \\
& Path B1 & -0.58 & -0.51 & -0.60 & -0.61 \\
\hline \multirow{2}{*}{$\operatorname{Pd}(111)$} & Path A2 & -0.53 & -0.55 & -0.53 & -0.59 \\
& Path B2 & -0.60 & -0.64 & -0.60 & -0.59 \\
$\operatorname{Pd}(211)$ & Path A2 & -0.51 & -0.51 & -0.52 & -0.56 \\
& Path B2 & -0.55 & -0.60 & -0.55 & -0.56 \\
\hline
\end{tabular}

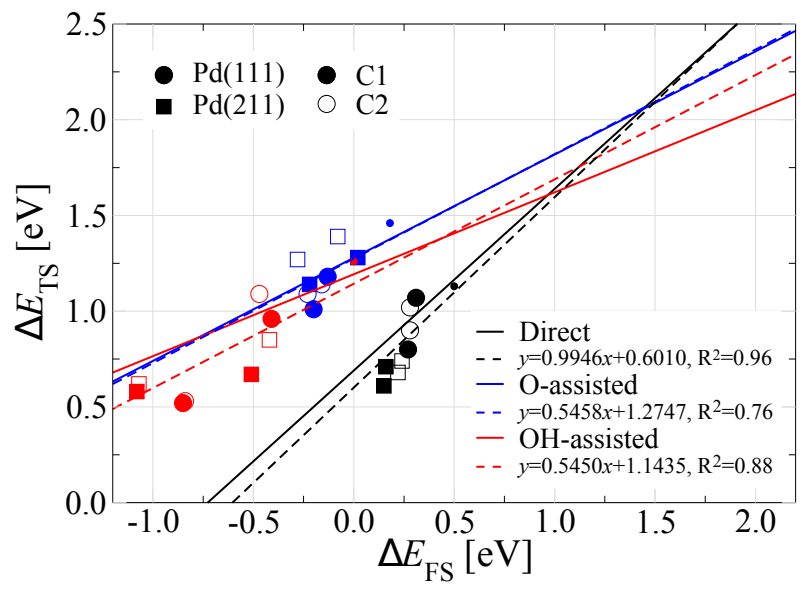

Figure S8. TSS relations for $\mathrm{C}_{3} \mathrm{H}_{8(\mathrm{~g})}$ dehydrogenation on clean, $\mathrm{O}^{*}$ -covered, and $\mathrm{OH}^{*}$-covered $\mathrm{Pd}(111)$ surfaces. Solid lines and small circles were taken from Yoo et al. ${ }^{4}$ Dashed lines represent the re-scaling relations using the results presented here and the data from Yoo et al. ${ }^{4}$ Legends description as in Figure 5. 


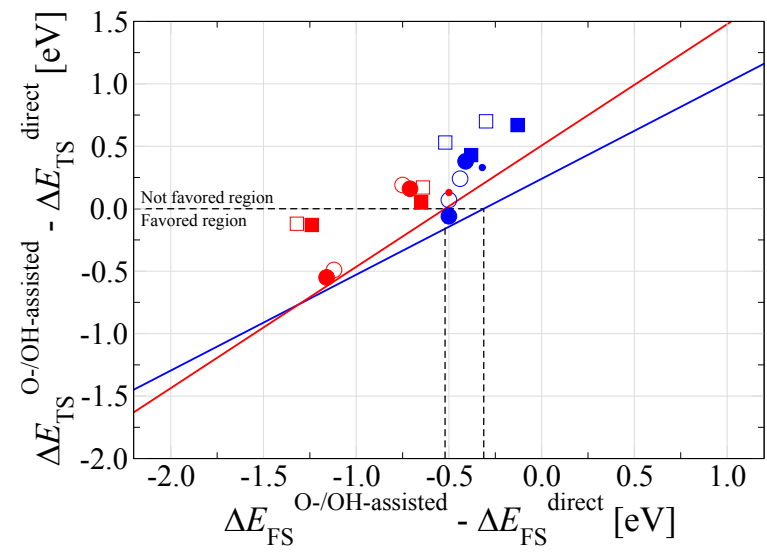

Figure S9. Difference in $\Delta E_{\mathrm{TS}}$ between the O-(blue) or OH-(green) assisted, and the direct $\mathrm{C}_{3} \mathrm{H}_{8}$ dehydrogenation reactions as a function of the difference in $\Delta E_{\mathrm{FS}}$. Legends descriptions as in Figure 5 Solid lines taken from Yoo et al. ${ }^{4}$ for $\mathrm{CH}_{4}$ activation on (111) surfaces.

Table S6. Final $\left(\Delta E_{\mathrm{FS}}\right)$ and transition state $\left(\Delta E_{\mathrm{TS}}\right)$ energies for dehydrogenation of propane reaction on clean, $\mathrm{O}^{*}$, and $\mathrm{OH}^{*}$ pre-covered Pd surfaces.

\begin{tabular}{|c|c|c|c|c|c|}
\hline & \multicolumn{2}{|c|}{$\operatorname{Pd}(111)$} & \multicolumn{2}{|c|}{$\operatorname{Pd}(211)$} \\
\hline & & $\Delta \boldsymbol{E}_{\mathbf{F S}}[\mathrm{eV}]$ & $\Delta \boldsymbol{E}_{\mathrm{TS}}[\mathrm{eV}]$ & $\Delta \boldsymbol{E}_{\mathbf{F S}}[\mathrm{eV}]$ & $\Delta \boldsymbol{E}_{\mathrm{TS}}[\mathrm{eV}]$ \\
\hline & $\mathrm{DH}$ & \multicolumn{4}{|c|}{ Direct } \\
\hline \multirow[t]{2}{*}{$\mathrm{C} 1$} & $1^{\text {st }}$ & 0.27 & 0.80 & 0.15 & 0.61 \\
\hline & $2^{\text {nd }}$ & 0.31 & 1.07 & 0.16 & 0.71 \\
\hline \multirow[t]{3}{*}{$\mathrm{C} 2$} & $1^{\text {st }}$ & 0.28 & 1.02 & 0.24 & 0.74 \\
\hline & $2^{\text {nd }}$ & 0.28 & 0.90 & 0.22 & 0.68 \\
\hline & $\mathrm{DH}$ & \multicolumn{4}{|c|}{ O-assisted } \\
\hline \multirow[t]{2}{*}{$\mathrm{C} 1$} & $1^{\text {st }}$ & -0.13 & 1.18 & 0.02 & 1.28 \\
\hline & $2^{\text {nd }}$ & -0.20 & 1.01 & -0.22 & 1.14 \\
\hline \multirow[t]{3}{*}{$\mathrm{C} 2$} & $1^{\text {st }}$ & -0.23 & 1.09 & -0.28 & 1.27 \\
\hline & $2^{\text {nd }}$ & -0.16 & 1.14 & -0.08 & 1.39 \\
\hline & DH & & $\mathrm{OH}-\mathrm{a}$ & isted & \\
\hline \multirow[t]{2}{*}{$\mathrm{C} 1$} & $1^{\text {st }}$ & -0.41 & 0.96 & -0.51 & 0.67 \\
\hline & $2^{\text {nd }}$ & -0.85 & 0.52 & -1.08 & 0.58 \\
\hline \multirow[t]{2}{*}{$\mathrm{C} 2$} & $1^{\text {st }}$ & -0.47 & 1.09 & -0.42 & 0.85 \\
\hline & $2^{\text {nd }}$ & -0.84 & 0.53 & -1.07 & 0.62 \\
\hline
\end{tabular}

\section{REFERENCES}

(1) Orita, H.; Itoh, N.; Inada, Y. A Comparison of CO Adsorption on $\mathrm{Pt}(211), \mathrm{Ni}(211)$, and Pd(211) Surfaces Using Density Functional Theory. Surf. Sci. 2004, 571 (1-3), 161-172. https://doi.org/10.1016/j.susc.2004.08.019.

(2) Lin, S.; Ma, J.; Zhou, L.; Huang, C.; Xie, D.; Guo, H. Influence of Step Defects on Methanol Decomposition: Periodic Density Functional Studies on $\operatorname{Pd}(211)$ and Kinetic Monte Carlo Simulations. J. Phys. Chem. C 2013, 117 (1), 451-459. https://doi.org/10.1021/jp310600q.

Pan, X.; Cai, Q.; Chen, W.; Zhuang, G.; Li, X.; Wang, J. A DFT Study of Gas Molecules Adsorption on the Anatase (001) Nanotube Arrays. Comput. Mater. Sci. 2013, 67, 174-181. https://doi.org/10.1016/j.commatsci.2012.09.006.

Yoo, J. S.; Khan, T. S.; Abild-Pedersen, F.; Nørskov, J. K.; Studt, F. On the Role of the Surface Oxygen Species during A-H (A = C, N, O) Bond Activation: A Density Functional Theory Study. Chem. Commun. 2015, 51 (13), 2621-2624. https://doi.org/10.1039/C4CC08658A.

(5) Tsai, C.; Latimer, A. A.; Yoo, J. S.; Studt, F.; Abild-Pedersen, F. Predicting Promoter-Induced Bond Activation on Solid Catalysts Using Elementary Bond Orders. J. Phys. Chem. Lett. 2015, 6 (18), 3670-3674. https://doi.org/10.1021/acs.jpclett.5b01792.

(6) Liu, L.; Fan, F.; Jiang, Z.; Gao, X.; Wei, J.; Fang, T. Mechanistic Study of $\mathrm{Pd}-\mathrm{Cu}$ Bimetallic Catalysts for Methanol Synthesis from CO2 Hydrogenation. J. Phys. Chem. C 2017, 121 (47), 26287-26299. https://doi.org/10.1021/acs.jpcc.7b06166.

Weaver, J. F.; Hakanoglu, C.; Hawkins, J. M.; Asthagiri, A. Molecular Adsorption of Small Alkanes on a PdO(101) Thin Film: Evidence of $\sigma$-Complex Formation. J. Chem. Phys. 2010, 132 (2), 024709. https://doi.org/10.1063/1.3277672.

Yang, M. L.; Zhu, Y. A.; Zhou, X. G.; Sui, Z. J.; Chen, D. FirstPrinciples Calculations of Propane Dehydrogenation over PtSn Catalysts. ACS Catal. 2012, 2 (6), 1247-1258. https://doi.org/10.1021/cs300031d.

Cao, X. Insight into Mechanism and Selectivity of Propane Dehydrogenation over the Pd-Doped Cu(111) Surface. RSC Adv. 2016, 6 (70), 65524-65532. https://doi.org/10.1039/C6RA15038A.

Sun, G.; Zhao, Z.-J.; Mu, R.; Zha, S.; Li, L.; Chen, S.; Zang, K.; Luo, J.; Li, Z.; Purdy, S. C.; et al. Breaking the Scaling Relationship via Thermally Stable $\mathrm{Pt} / \mathrm{Cu}$ Single Atom Alloys for Catalytic Dehydrogenation. Nat. Commun. 2018, 9 (1), 4454. https://doi.org/10.1038/s41467-018-06967-8.

e Silva, T. L. S.; Schmal, M. Adsorption and Desorption of Propane on Pd (111): A van Der Waals Density Functional Study. Energy Binding Sites and Geometries. Surf. Sci. 2017, 664, 8286. https://doi.org/10.1016/j.susc.2017.06.004.

Zha, S.; Sun, G.; Wu, T.; Zhao, J.; Zhao, Z.-J.; Gong, J. Identification of Pt-Based Catalysts for Propane Dehydrogenation via a Probability Analysis. Chem. Sci. 2018, 9 (16), 3925-3931. https://doi.org/10.1039/C8SC00802G. 


\section{Cartesian coordinates of optimized structures including frequencies}

Below optimized structures of the palladium surfaces and the gas phase molecules are provided in cif-format. Additionally, the electronic energies, the zero-point vibrational energy (both in $\mathrm{eV}$ ) and the list of frequencies $\left(\mathrm{cm}^{-1}\right)$ are provided. Some of the loosely bound olefins exhibit an imaginary harmonic frequency resulting from soft vibrational modes and small numerical inaccuracies of the finite difference scheme for force constants.

The notation used is the following: Pd1.A1.P, where the first part stand for the Pd surfaces (Pd1 or Pd2), the second part represent the type of the dehydrogenation (direct $(\mathbf{A})$ or oxidative $(\mathbf{B})$ ) and the route (1-propyl (1) or 2-propyl (2)) and the last part stands for the adsorbate (propane (P), 1-propyl (1p), 2-propyl (2p), and, propylene (y)) or the transition state (TS1 (T1), TS2 (T2), TS3 (T3) and, TS4 (T4)), this notation follows the same presented in the main text of this paper (see Scheme 1, Figures 1 and 2). For OH-assisted dehydrogenation $\mathbf{C}$ is used as label and for the transition states is used T5. The Pd surfaces and adsorption of oxygen species are label as Pd111/Pd211.Ad.O/OH.

\# Pd1.A1.P; $E=-114.38 ; \mathrm{ZPVE}=2.77 \mathrm{eV}$; frequencies=17.13i, 22.96i, 3056.00, 3053.57, 3045.15, 3028.49, 2985.05, 2979.65, 2968.54, 2954.77, 1493.36, 1489.58, 1478.70, 1469.43, 1464.87, 1402.26, 1381.61, 1342.50, 1296.41, 1192.38, 1155.07, 1043.72, 918.65, 898.82, $858.26,746.45,368.07,268.71,218.19,47.49,20.07,10.77,2.06 \mathrm{~cm}^{\wedge}-1$

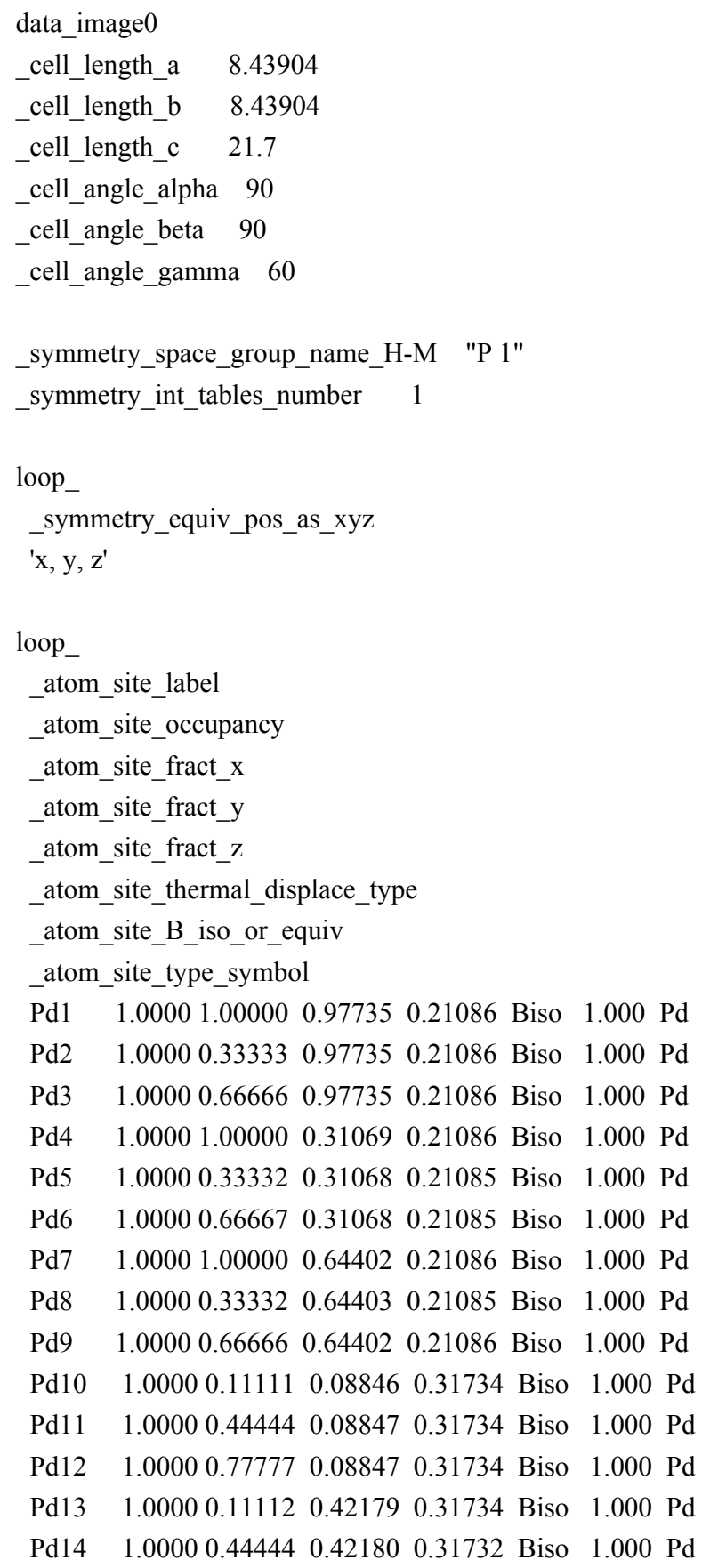




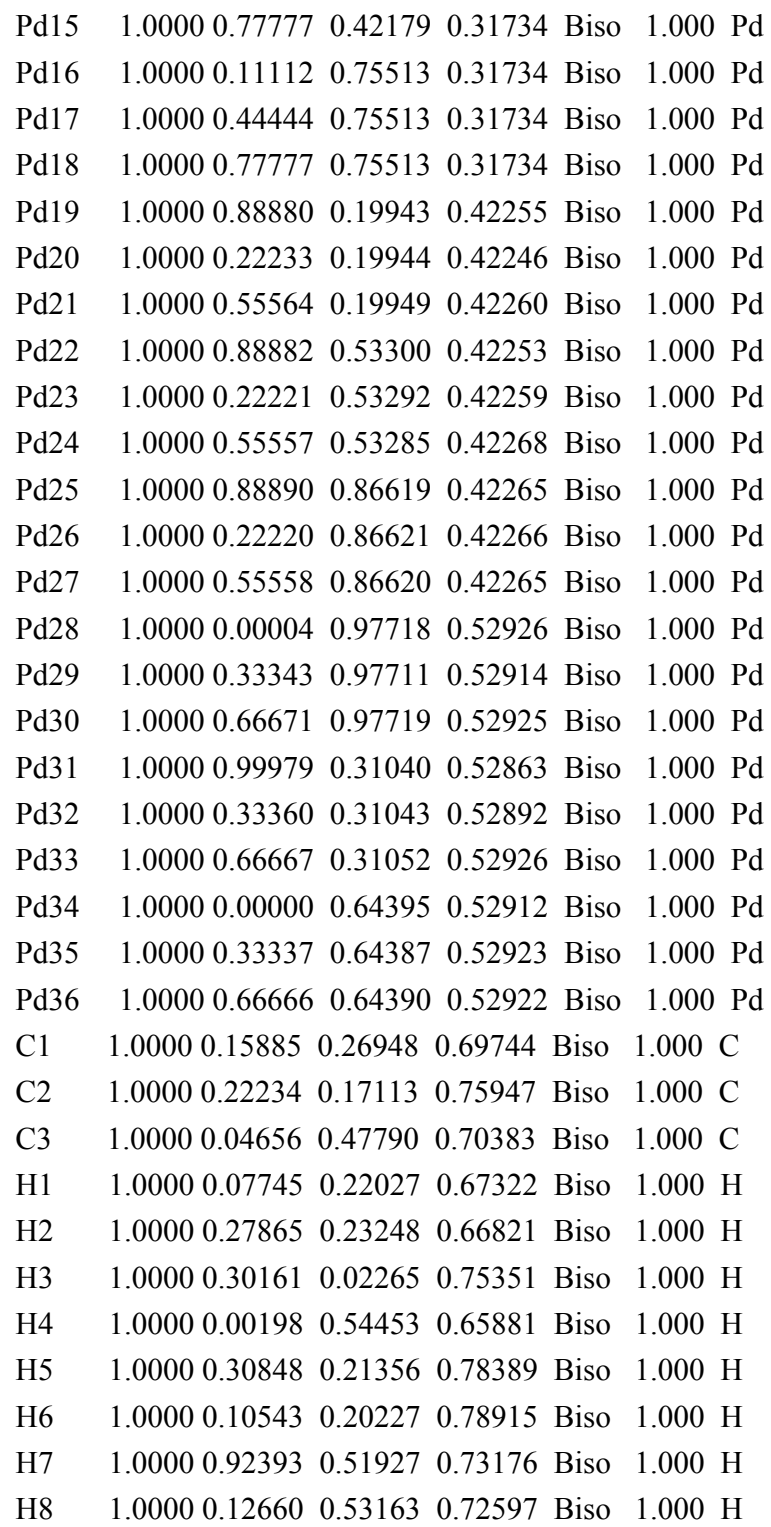

\# Pd1.A1.T1; E= -113.38; ZPVE=2.60 eV; frequencies=876.90i, 3076.91, 3064.87, 3063.17, 3022.03, 2995.37, 2989.58, 2984.10, 1489.77, $1479.87,1470.45,1452.95,1407.16,1388.82,1317.40,1290.43,1204.36,1124.37,1063.56,997.15,960.44,884.01,799.01,699.29,515.82$, $339.82,246.39,220.74,173.98,97.61,66.00,40.49,23.78 \mathrm{~cm}^{\wedge}-1$

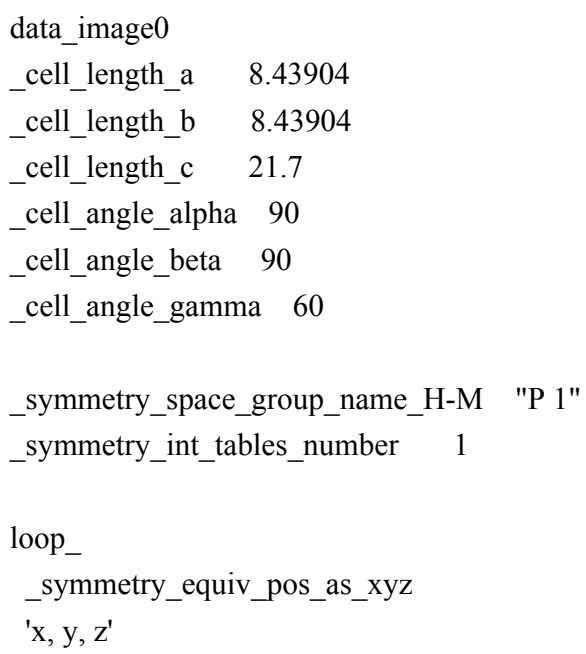




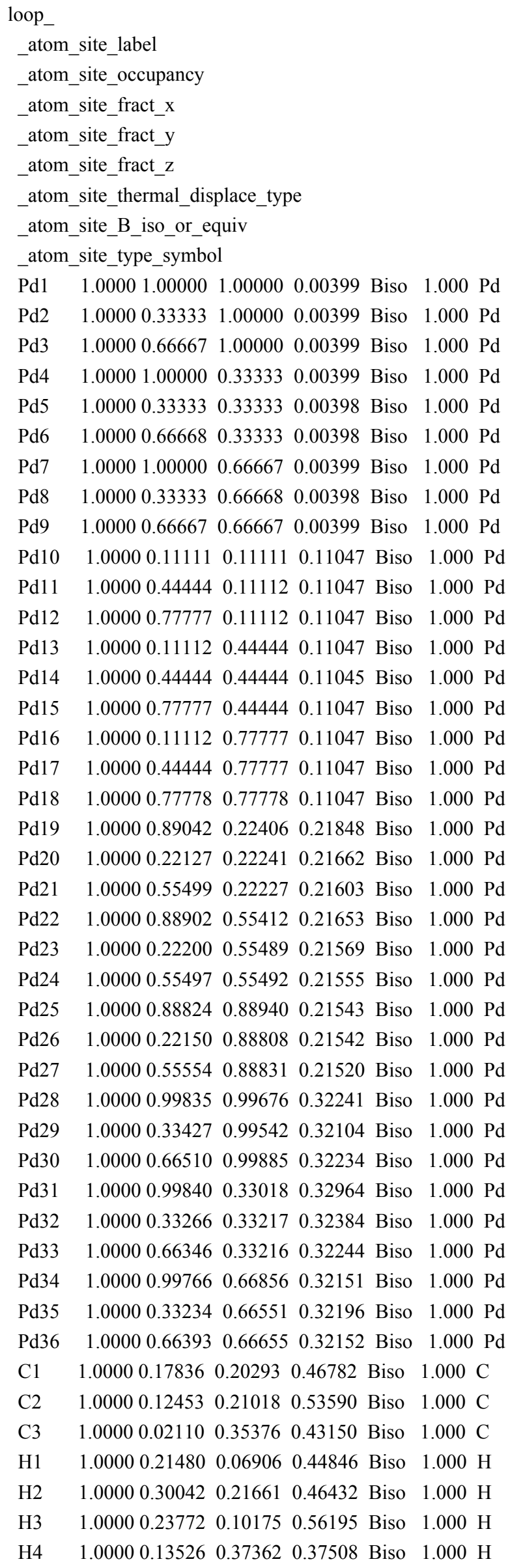


H5 1.00000 .090890 .341290 .55681 Biso $1.000 \mathrm{H}$

H6 $\quad 1.00000 .005710 .191860 .54088$ Biso $1.000 \mathrm{H}$

H7 $\quad \begin{array}{lllllll}1.0000 & 0.89790 & 0.34161 & 0.43297 & \text { Biso } & 1.000 \mathrm{H}\end{array}$

H8 1.00000 .986070 .490960 .44571 Biso $1.000 \mathrm{H}$

\# Pd1.A1.1p; $\mathrm{E}=-113.89 ; \mathrm{ZPVE}=2.64 \mathrm{eV}$; frequencies=3070.62, 3067.30, 3059.08, 3020.45, 2992.73, 2988.56, 2973.58, 1489.09, 1476.82, $1467.57,1434.45,1382.30,1304.48,1289.62,1202.53,1109.61,1025.08,992.08,975.91,953.93,939.37,884.40,776.75,671.60,643.44$, $526.45,238.39,234.64,159.58,114.84,52.48,39.04,23.66 \mathrm{~cm}^{\wedge}-1$

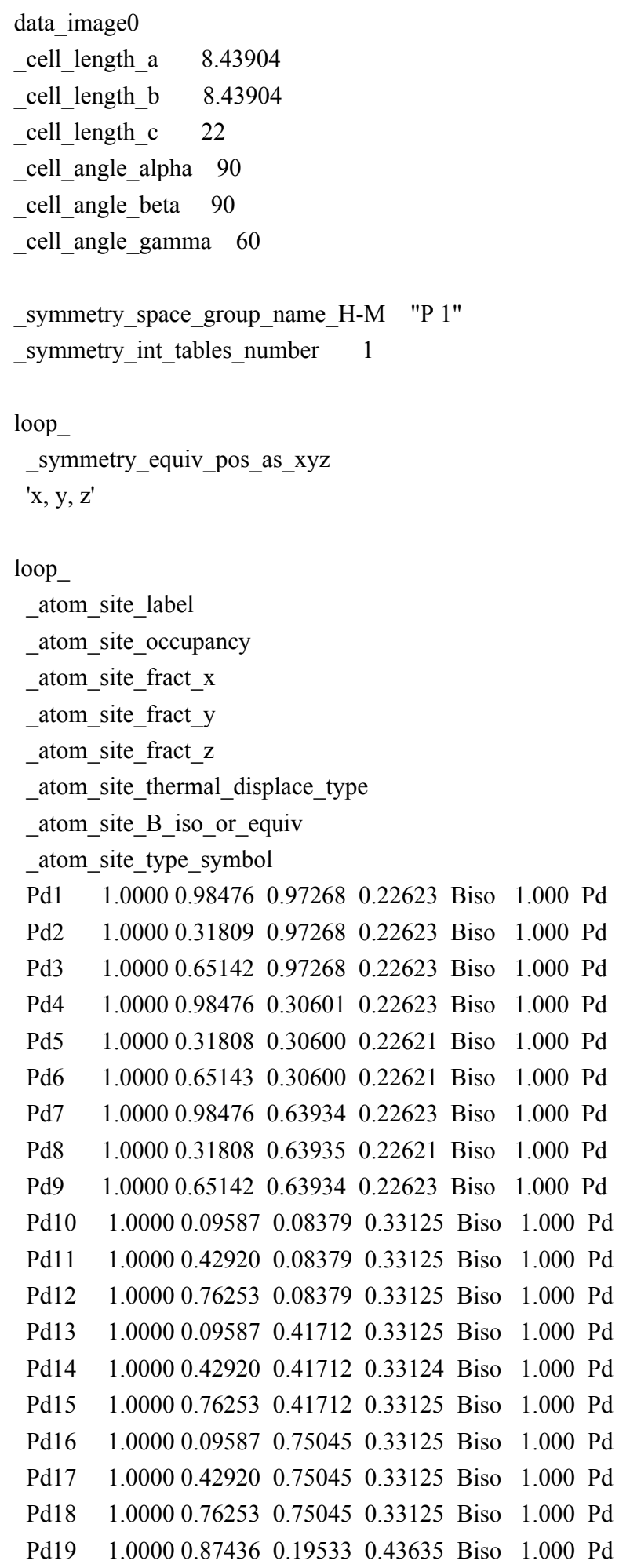




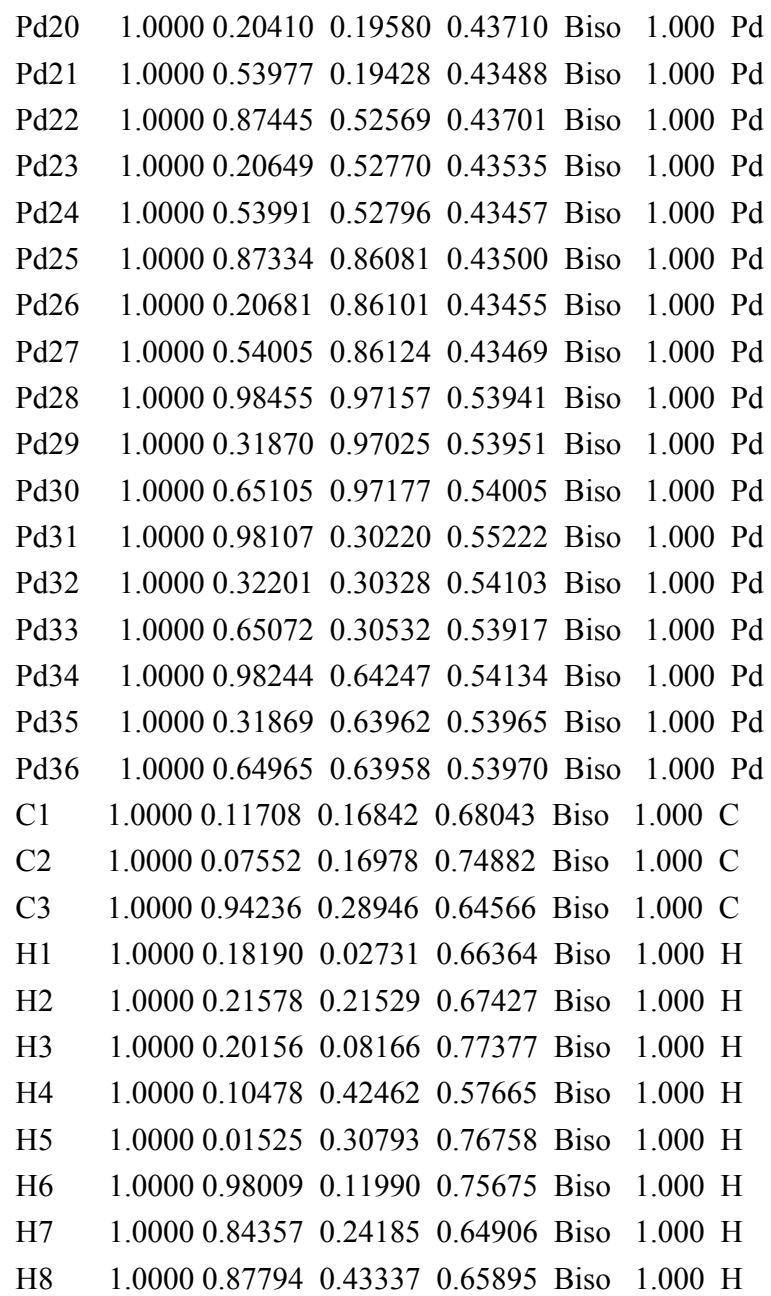

\# Pd1.A1.T2; $\mathrm{E}=-113.14 ; \mathrm{ZPVE}=2.45 \mathrm{eV}$; frequencies=895.71i, 3161.10, 3092.51, 3075.39, 3068.67, 3058.91, 2988.63, 1483.93, 1475.54, 1459.61, 1393.00, 1369.38, 1219.13, 1192.06, 1132.30, 1081.24, 1018.32, 1003.67, 957.68, 940.13, 905.81, 888.76, 720.18, 604.95, 548.15, $413.24,376.67,301.45,198.75,148.02,94.42,70.73,51.34 \mathrm{~cm}^{\wedge}-1$

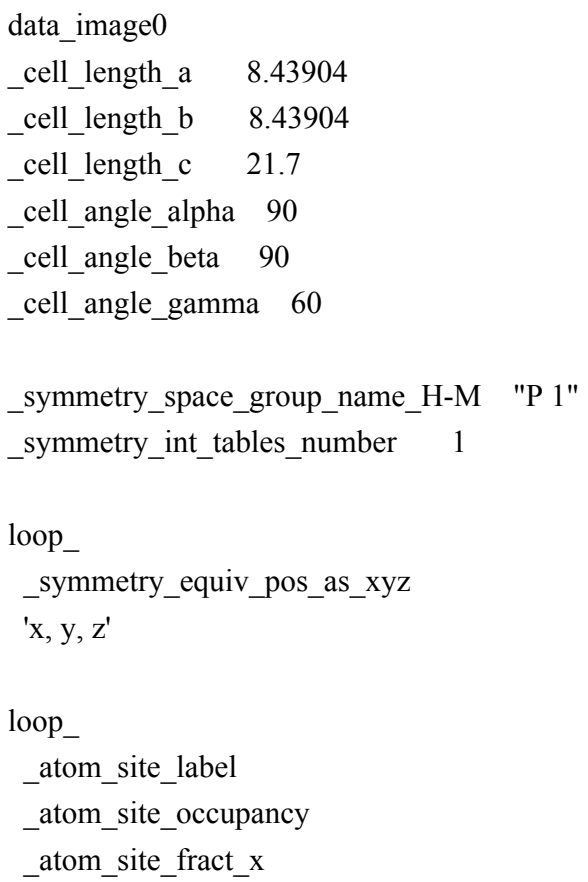




\begin{tabular}{|c|c|c|c|}
\hline \multicolumn{4}{|c|}{$\begin{array}{l}\text { atom_site_fract_y } \\
\text {-atom_site_fract_z } \\
\text { atom_site_thermal_displace_type } \\
\text { atom_site_B_iso_or_equiv } \\
\text { atom_site_type_symbol }\end{array}$} \\
\hline Pd1 & 1.00001 .000001 .00000 & 0.00399 Biso & $1.000 \mathrm{Pd}$ \\
\hline $\mathrm{Pd} 2$ & 1.00000 .333331 .00000 & 0.00399 Biso & $1.000 \mathrm{Pd}$ \\
\hline $\mathrm{Pd} 3$ & $1.00000 .66667 \quad 1.00000$ & 0.00399 Biso & $1.000 \mathrm{Pd}$ \\
\hline $\mathrm{Pd} 4$ & $1.00001 .00000 \quad 0.33333$ & 0.00399 Biso & $1.000 \mathrm{Pd}$ \\
\hline Pd5 & 1.00000 .333330 .33333 & 0.00398 Biso & $1.000 \mathrm{Pd}$ \\
\hline Pd6 & $1.0000 \quad 0.666680 .33333$ & 0.00398 Biso & $1.000 \mathrm{Pd}$ \\
\hline $\operatorname{Pd} 7$ & $1.00001 .00000 \quad 0.66667$ & 0.00399 Biso & $1.000 \mathrm{Pd}$ \\
\hline $\operatorname{Pd} 8$ & $\begin{array}{lll}1.0000 & 0.33333 & 0.66668\end{array}$ & 0.00398 Biso & $1.000 \mathrm{Pd}$ \\
\hline Pd9 & 1.00000 .666670 .66667 & 0.00399 Biso & $1.000 \mathrm{Pd}$ \\
\hline $\operatorname{Pd} 10$ & 1.00000 .111110 .11111 & 0.11047 Biso & $1.000 \mathrm{Pd}$ \\
\hline Pd11 & 1.00000 .444440 .11112 & 0.11047 Biso & $1.000 \mathrm{Pd}$ \\
\hline $\operatorname{Pd} 12$ & 1.00000 .777770 .11112 & 0.11047 Biso & $1.000 \mathrm{Pd}$ \\
\hline $\operatorname{Pd} 13$ & 1.00000 .111120 .44444 & 0.11047 Biso & $1.000 \mathrm{Pd}$ \\
\hline Pd14 & $1.0000 \quad 0.44444 \quad 0.44444$ & 0.11045 Biso & $1.000 \mathrm{Pd}$ \\
\hline $\operatorname{Pd} 15$ & $\begin{array}{lll}1.0000 & 0.77777 & 0.44444\end{array}$ & 0.11047 Biso & $1.000 \mathrm{Pd}$ \\
\hline Pd16 & 1.00000 .111120 .77777 & 0.11047 Biso & $1.000 \mathrm{Pd}$ \\
\hline $\operatorname{Pd} 17$ & $\begin{array}{lll}1.0000 & 0.44444 & 0.77777\end{array}$ & 0.11047 Biso & $1.000 \mathrm{Pd}$ \\
\hline $\operatorname{Pd} 18$ & $\begin{array}{lll}1.0000 & 0.77778 & 0.77778\end{array}$ & 0.11047 Biso & $1.000 \mathrm{Pd}$ \\
\hline Pd19 & $\begin{array}{lll}1.0000 & 0.88906 & 0.22398\end{array}$ & 0.21670 Biso & $1.000 \mathrm{Pd}$ \\
\hline $\operatorname{Pd} 20$ & $\begin{array}{lll}1.0000 & 0.21690 & 0.22423\end{array}$ & 0.21856 Biso & $1.000 \mathrm{Pd}$ \\
\hline $\operatorname{Pd} 21$ & $\begin{array}{lll}1.0000 & 0.55408 & 0.22223\end{array}$ & 0.21521 Biso & $1.000 \mathrm{Pd}$ \\
\hline $\operatorname{Pd} 22$ & $\begin{array}{lll}1.0000 & 0.88961 & 0.55253\end{array}$ & 0.21835 Biso & $1.000 \mathrm{Pd}$ \\
\hline $\operatorname{Pd} 23$ & $\begin{array}{lll}1.0000 & 0.22096 & 0.55608\end{array}$ & 0.21573 Biso & $1.000 \mathrm{Pd}$ \\
\hline $\operatorname{Pd} 24$ & $\begin{array}{lll}1.0000 & 0.55469 & 0.55622\end{array}$ & 0.21497 Biso & $1.000 \mathrm{Pd}$ \\
\hline $\operatorname{Pd} 25$ & $\begin{array}{lll}1.0000 & 0.88740 & 0.88897\end{array}$ & 0.21518 Biso & $1.000 \mathrm{Pd}$ \\
\hline $\operatorname{Pd} 26$ & $\begin{array}{lll}1.0000 & 0.22182 & 0.88925\end{array}$ & 0.21513 Biso & $1.000 \mathrm{Pd}$ \\
\hline $\operatorname{Pd} 27$ & $\begin{array}{lll}1.0000 & 0.55406 & 0.88931\end{array}$ & 0.21604 Biso & $1.000 \mathrm{Pd}$ \\
\hline $\operatorname{Pd} 28$ & $1.00000 .99750 \quad 0.99913$ & 0.32004 Biso & $1.000 \mathrm{Pd}$ \\
\hline $\operatorname{Pd} 29$ & $1.0000 \quad 0.332640 .99865$ & 0.32361 Biso & $1.000 \mathrm{Pd}$ \\
\hline $\operatorname{Pd} 30$ & $\begin{array}{lll}1.0000 & 0.66461 & 0.00131\end{array}$ & 0.32225 Biso & $1.000 \mathrm{Pd}$ \\
\hline Pd31 & $\begin{array}{lll}1.0000 & 0.99074 & 0.33911\end{array}$ & 0.33971 Biso & $1.000 \mathrm{Pd}$ \\
\hline $\operatorname{Pd} 32$ & $\begin{array}{lll}1.0000 & 0.33684 & 0.33419\end{array}$ & 0.32225 Biso & $1.000 \mathrm{Pd}$ \\
\hline Pd33 & $\begin{array}{lll}1.0000 & 0.66368 & 0.33447\end{array}$ & 0.32034 Biso & $1.000 \mathrm{Pd}$ \\
\hline Pd34 & $\begin{array}{lll}1.0000 & 0.99578 & 0.67425\end{array}$ & 0.32314 Biso & $1.000 \mathrm{Pd}$ \\
\hline Pd35 & 1.00000 .333390 .66833 & 0.32122 Biso & $1.000 \mathrm{Pd}$ \\
\hline $\operatorname{Pd} 36$ & 1.00000 .663600 .66893 & 0.32191 Biso & $1.000 \mathrm{Pd}$ \\
\hline $\mathrm{C} 1$ & 1.00000 .073960 .19795 & 0.44395 Biso & $1.000 \mathrm{C}$ \\
\hline $\mathrm{C} 2$ & $1.0000 \quad 0.233160 .18889$ & 0.47934 Biso & $1.000 \mathrm{C}$ \\
\hline $\mathrm{C} 3$ & $\begin{array}{lll}1.0000 & 0.90884 & 0.36908\end{array}$ & 0.43578 Biso & $1.000 \mathrm{C}$ \\
\hline H1 & $\begin{array}{lll}1.0000 & 0.05552 & 0.07989\end{array}$ & 0.44951 Biso & $1.000 \mathrm{H}$ \\
\hline $\mathrm{H} 2$ & $\begin{array}{lll}1.0000 & 0.16450 & 0.12869\end{array}$ & 0.38186 Biso & $1.000 \mathrm{H}$ \\
\hline H3 & $\begin{array}{lll}1.0000 & 0.35942 & 0.05977\end{array}$ & 0.47149 Biso & $1.000 \mathrm{H}$ \\
\hline H4 & $1.0000 \quad 0.12474 \quad 0.46354$ & 0.35963 Biso & $1.000 \mathrm{H}$ \\
\hline H5 & $\begin{array}{lll}1.0000 & 0.25620 & 0.30092\end{array}$ & 0.46662 Biso & $1.000 \mathrm{H}$ \\
\hline H6 & $\begin{array}{lll}1.0000 & 0.20119 & 0.20027\end{array}$ & 0.52864 Biso & $1.000 \mathrm{H}$ \\
\hline H7 & $\begin{array}{lll}1.0000 & 0.77895 & 0.37151\end{array}$ & 0.43681 Biso & $1.000 \mathrm{H}$ \\
\hline $\mathrm{H} 8$ & 1.00000 .904470 .49335 & 0.45084 Biso & $1.000 \mathrm{H}$ \\
\hline
\end{tabular}


\# Pd1.A1.y; $\mathrm{E}=-113.88 ; \mathrm{ZPVE}=2.49 \mathrm{eV}$; frequencies=3164.35, 3093.95, 3075.90, 3069.26, 3047.28, 2972.97, 1529.93, 1471.42, 1455.27, 1406.52, 1384.91, 1259.31, 1170.50, 1026.99, 1010.53, 967.76, 955.47, 947.26, 918.32, 911.17, 885.87, 825.80, 780.07, 699.54, 673.85, $405.82,326.26,220.08,199.67,131.31,78.61,60.22,14.64 \mathrm{~cm}^{\wedge}-1$

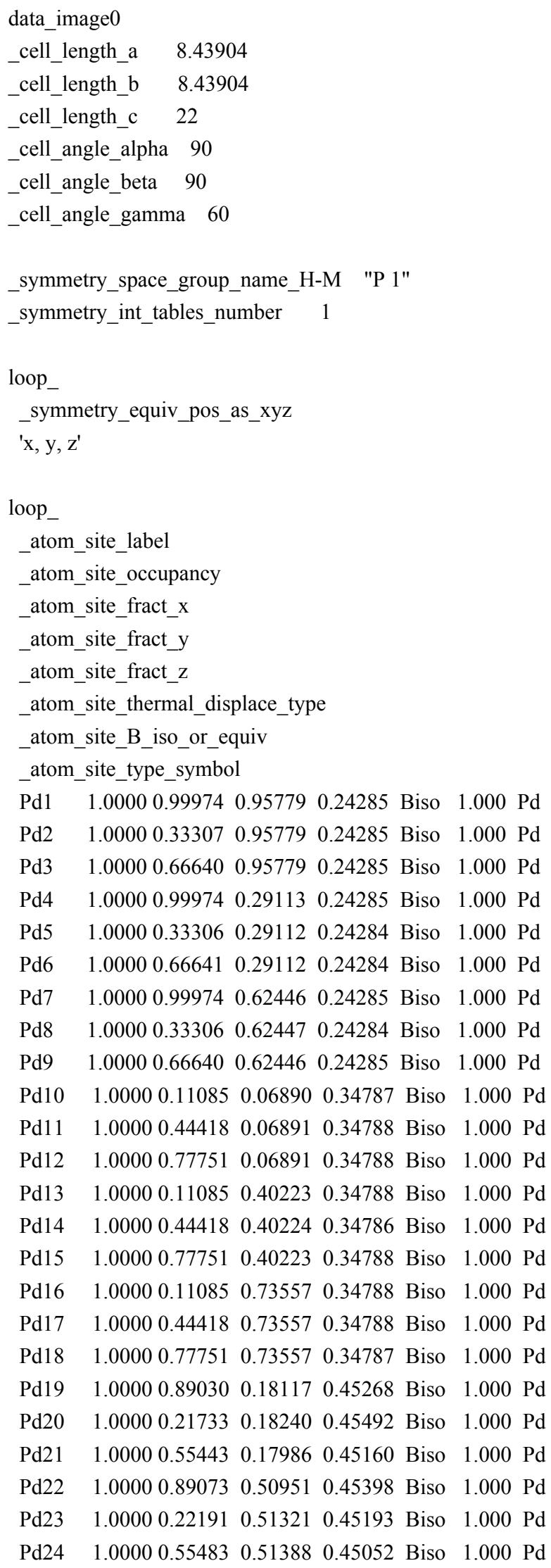




$\begin{array}{llllllll}\mathrm{Pd} 25 & 1.0000 & 0.88850 & 0.84646 & 0.45151 & \text { Biso } & 1.000 \mathrm{Pd} \\ \mathrm{Pd} 26 & 1.0000 & 0.22224 & 0.84685 & 0.45117 & \text { Biso } & 1.000 \mathrm{Pd} \\ \mathrm{Pd} 27 & 1.0000 & 0.55515 & 0.84671 & 0.45171 & \text { Biso } & 1.000 \mathrm{Pd} \\ \mathrm{Pd} 28 & 1.0000 & 0.00028 & 0.95669 & 0.55530 & \text { Biso } & 1.000 \mathrm{Pd} \\ \mathrm{Pd} 29 & 1.0000 & 0.33355 & 0.95464 & 0.55709 & \text { Biso } & 1.000 \mathrm{Pd} \\ \mathrm{Pd} 30 & 1.0000 & 0.67035 & 0.95632 & 0.55773 & \text { Biso } & 1.000 \mathrm{Pd} \\ \mathrm{Pd} 31 & 1.0000 & 0.99396 & 0.28950 & 0.57499 & \text { Biso } & 1.000 \mathrm{Pd} \\ \mathrm{Pd} 32 & 1.0000 & 0.33626 & 0.29267 & 0.55857 & \text { Biso } & 1.000 \mathrm{Pd} \\ \mathrm{Pd} 33 & 1.0000 & 0.66599 & 0.29156 & 0.55423 & \text { Biso } & 1.000 \mathrm{Pd} \\ \mathrm{Pd} 34 & 1.0000 & 0.99914 & 0.62857 & 0.55725 & \text { Biso } & 1.000 \mathrm{Pd} \\ \mathrm{Pd} 35 & 1.0000 & 0.33449 & 0.62550 & 0.55567 & \text { Biso } & 1.000 \mathrm{Pd} \\ \mathrm{Pd} 36 & 1.0000 & 0.66500 & 0.62512 & 0.55541 & \text { Biso } & 1.000 \mathrm{Pd} \\ \mathrm{C} 1 & 1.0000 & 0.01150 & 0.19601 & 0.67715 & \text { Biso } & 1.000 \mathrm{C} \\ \mathrm{C} 2 & 1.0000 & 0.18311 & 0.16284 & 0.70882 & \text { Biso } & 1.000 \mathrm{C} \\ \mathrm{C} 3 & 1.0000 & 0.85932 & 0.36776 & 0.66928 & \text { Biso } & 1.000 \mathrm{C} \\ \mathrm{H} 1 & 1.0000 & 0.99234 & 0.07801 & 0.67333 & \text { Biso } & 1.000 \mathrm{H} \\ \mathrm{H} 2 & 1.0000 & 0.44777 & 0.06412 & 0.59252 & \text { Biso } & 1.000 \mathrm{H} \\ \mathrm{H} 3 & 1.0000 & 0.30429 & 0.04219 & 0.69127 & \text { Biso } & 1.000 \mathrm{H} \\ \mathrm{H} 4 & 1.0000 & 0.11784 & 0.41340 & 0.59394 & \text { Biso } & 1.000 \mathrm{H} \\ \mathrm{H} 5 & 1.0000 & 0.20391 & 0.28019 & 0.70543 & \text { Biso } & 1.000 \mathrm{H} \\ \mathrm{H} 6 & 1.0000 & 0.17118 & 0.13780 & 0.75714 & \text { Biso } & 1.000 \mathrm{H} \\ \mathrm{H} 7 & 1.0000 & 0.72463 & 0.38279 & 0.66357 & \text { Biso } & 1.000 \mathrm{H} \\ \mathrm{H} 8 & 1.0000 & 0.86263 & 0.48954 & 0.68242 & \text { Biso } & 1.000 \mathrm{H}\end{array}$

\# Pd1.B1.P; $E=-114.46 ; Z P V E=2.77$ eV; frequencies=16.16i, 3051.41, 3048.32, 3044.69, 3036.85, 2994.17, 2978.29, 2974.90, 2894.23, $1490.65,1488.52,1477.41,1470.82,1455.27,1401.44,1382.87,1339.40,1289.45,1188.27,1155.76,1042.59,919.82,897.65,857.87$, $736.14,368.14,274.13,221.63,59.27,51.74,44.79,17.31,6.10 \mathrm{~cm}^{\wedge}-1$

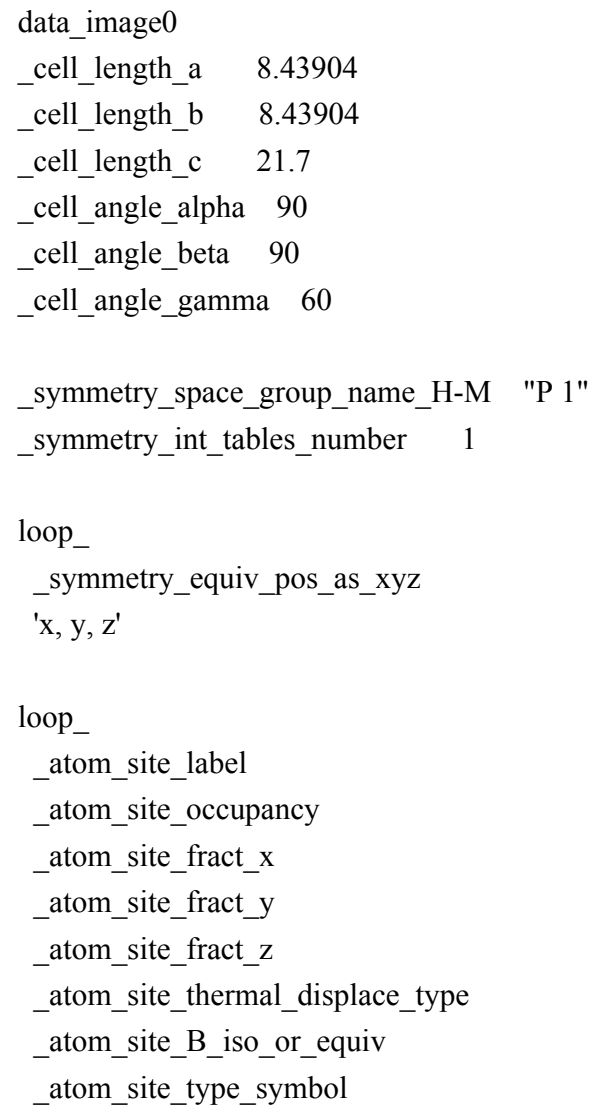




\begin{tabular}{|c|c|c|c|c|c|}
\hline Pd1 & 1.00000 .99951 & 0.97765 & & Biso & 1.000 \\
\hline $\mathrm{Pd} 2$ & 1.00000 .33284 & 0.97765 & 0.22921 & Biso & 1.000 \\
\hline $\mathrm{Pd} 3$ & 1.00000 .66617 & 0.97765 & 0.22921 & Biso & 1.000 \\
\hline $\mathrm{Pd} 4$ & 1.00000 .99951 & 0.31099 & 0.22921 & Biso & 1.000 \\
\hline $\mathrm{Pd} 5$ & 1.00000 .33283 & 0.31098 & 0.22919 & Biso & 1.000 \\
\hline Pd6 & 1.00000 .66618 & 0.31098 & 0.22919 & Biso & 1.000 \\
\hline d7 & 1.00000 .99951 & 0.64432 & 0.22921 & Biso & 1.000 \\
\hline Pd8 & 1.00000 .33283 & 0.64433 & 0.22919 & Biso & 1.000 \\
\hline Pd9 & 1.00000 .66617 & 0.64432 & 0.22921 & Biso & 1.000 \\
\hline Pd10 & 1.00000 .11062 & 0.08876 & 0.33568 & Biso & 1.000 \\
\hline Pd11 & 1.00000 .44395 & 0.08877 & 0.33569 & Biso & 1.000 \\
\hline $\operatorname{Pd} 12$ & 1.00000 .77728 & 0.08877 & 0.33569 & Biso & 1.000 \\
\hline d13 & 1.00000 . & 0.42209 & 0.33569 & Biso & 1.000 \\
\hline Pd14 & 1.00000 .44395 & 0.42210 & 0.33567 & 7 Biso & 1.000 \\
\hline d15 & 1.00000 .77728 & 0.42209 & 0.33569 & Biso & 1.000 \\
\hline Pd16 & 1.00000 .11062 & 0.75543 & 0.33569 & Biso & 1.000 \\
\hline $\operatorname{Pd} 17$ & 1.00000 .44395 & 0.75543 & 0.33569 & Biso & 1.000 \\
\hline Pd18 & 1.00000 .77728 & 0.75543 & 0.33568 & Biso & 1.000 \\
\hline d19 & 1.00000 .88918 & 0.19960 & 0.44092 & 2 Biso & 1.000 \\
\hline $\operatorname{Pd} 20$ & 1.00000 .22223 & 0.19980 & 0.44113 & 3 Biso & 1.000 \\
\hline $\operatorname{Pd} 21$ & 1.00000 .55529 & 0.19973 & 0.44088 & Biso & 1.00 \\
\hline $\mathrm{Pd} 22$ & 1.00000 .88881 & 0.53309 & 0.44113 & 3 Biso & 1.00 \\
\hline $\operatorname{Pd} 23$ & 1.00000 .22215 & 0.53301 & 0.44104 & 4 Biso & 1.000 \\
\hline $\mathrm{Pd} 24$ & 1.00000 .55543 & 0.53334 & 0.44086 & 5 Biso & 1.000 \\
\hline $\mathrm{Pd} 25$ & 1.00000 .88896 & 0.86637 & 0.44106 & 5 Biso & 1.000 \\
\hline $\operatorname{Pd} 26$ & 1.00000 .22209 & 0.86661 & 0.44104 & 4 Biso & 1.000 \\
\hline $\operatorname{Pd} 27$ & 1.00000 .55540 & 0.86639 & 0.44108 & Biso & 1.00 \\
\hline $\operatorname{Pd} 28$ & 1.00000 .00049 & 0.97742 & 0.54778 & Biso & 1.00 \\
\hline $\operatorname{Pd} 29$ & 1.00000 .33378 & 0.97745 & 0.54779 & Biso & 1.00 \\
\hline $\mathrm{Pd} 30$ & 1.00000 .66730 & 0.97706 & 0.54742 & 2 Biso & 1.00 \\
\hline $\mathrm{Pd} 31$ & 1.00000 .00058 & 0.31072 & 0.54776 & 5 Biso & 1.000 \\
\hline $\mathrm{Pd} 32$ & 1.00000 .33391 & 0.31072 & 0.54777 & 7 Biso & 1.000 \\
\hline $\mathrm{Pd} 33$ & 1.00000 .66761 & 0.31072 & 0.54680 & Biso & 1.000 \\
\hline $\mathrm{Pd} 34$ & 1.00000 .00051 & 0.64403 & 0.54775 & 5 Biso & 1.000 \\
\hline $\mathrm{Pd} 35$ & 1.00000 .33359 & 0.64450 & 0.54734 & 4 Biso & 1.000 \\
\hline $\mathrm{Pd} 36$ & 1.00000 .66713 & 0.64407 & $7 \quad 0.54774$ & 4 Biso & 1.00 \\
\hline $\mathrm{C} 1$ & 1.00000 .47956 & 0.31934 & 0.71226 & Biso & 1.000 \\
\hline $\mathrm{C} 2$ & 1.00000 .48659 & 0.49540 & 0.72189 & Biso & 1.000 \\
\hline $\mathrm{C} 3$ & 1.00000 .66379 & 0.14582 & 0.72335 & Biso & 1.000 \\
\hline H1 & 1.00000 .43386 & 0.31714 & 0.66487 & Biso & 1.000 \\
\hline $\mathrm{H} 2$ & 1.00000 .37554 & 0.31952 & 0.74258 & Biso & 1.000 \\
\hline H3 & 1.00000 .35419 & 0.61693 & 0.71229 & Biso & 1.000 \\
\hline H4 & 1.00000 .65531 & 0.02233 & 0.71508 & Biso & 1.000 \\
\hline H5 & 1.00000 .52431 & 0.50558 & 0.76939 & Biso & 1.000 \\
\hline H6 & 1.00000 .58813 & 0.49947 & 0.69148 & Biso & 1.000 \\
\hline $\mathrm{H} 7$ & 1.00000 .76939 & 0.14091 & 0.69272 & Biso & 1.000 \\
\hline UP & 1.00000 .71016 & 0.14038 & 0.77012 & Biso & 1.000 \\
\hline
\end{tabular}

\# Pd1.B1.T1; $E=-113.26 ; Z P V E=2.60 \mathrm{eV}$; frequencies= 892.22i, 3073.82, 3054.94, 3036.98, 3021.02, 3008.09, 2962.37, 2918.87, 1533.77, $1479.04,1463.08,1455.93,1450.03,1392.60,1374.28,1323.84,1193.80,1126.27,1099.06,1006.09,930.97,922.38,852.75,470.16$, $396.28,351.73,277.30,229.87,185.44,116.82,86.28,54.52,20.72 \mathrm{~cm}^{\wedge}-1$ 


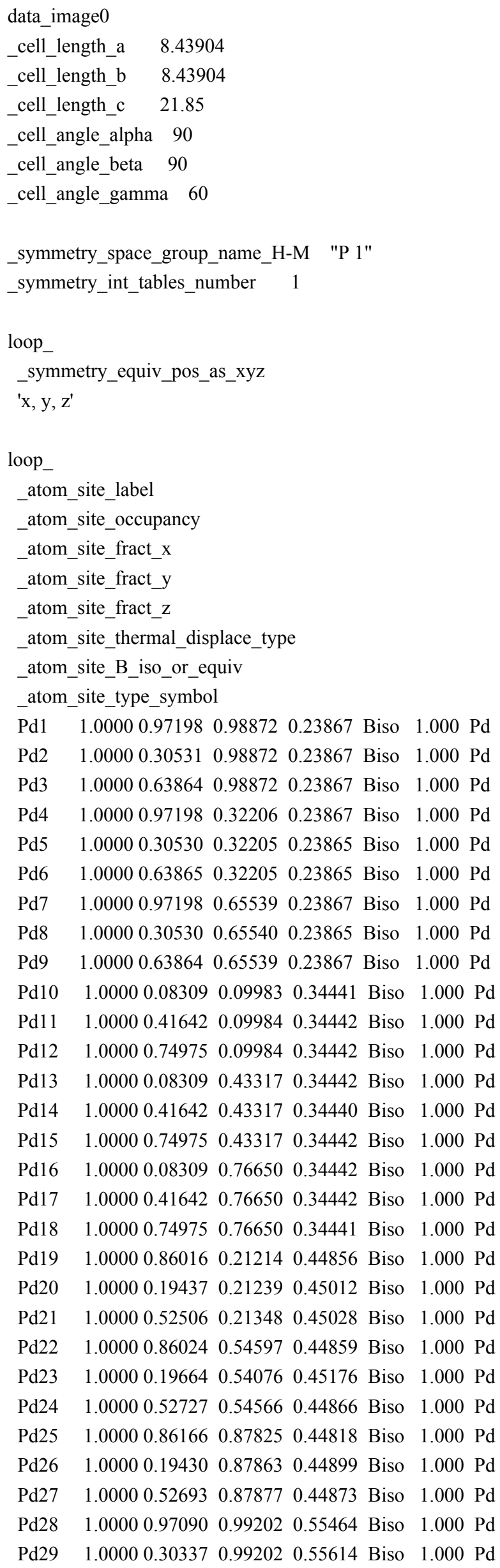




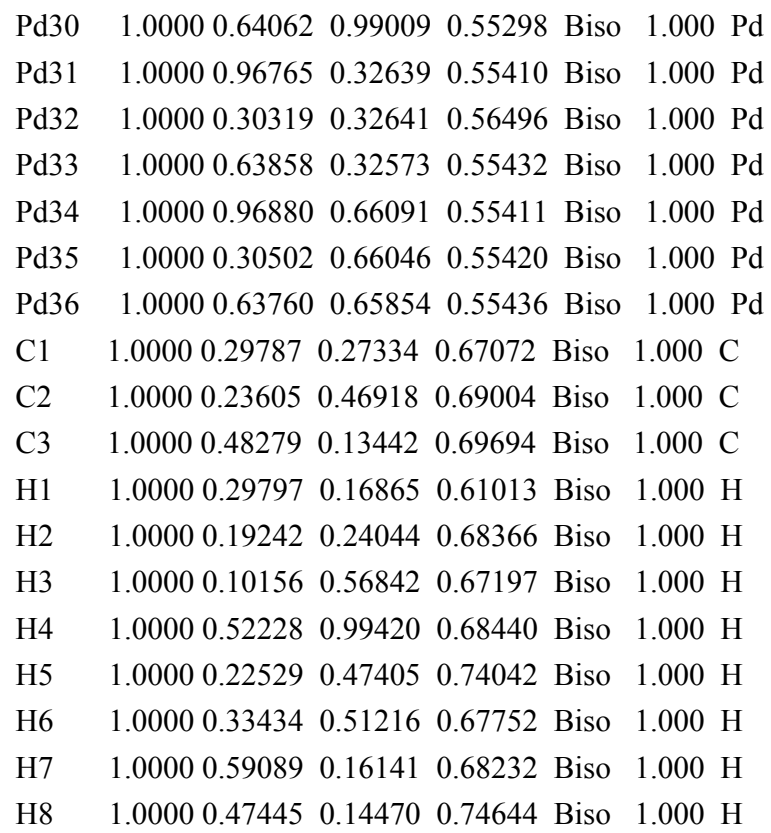

\# Pd1.B1.2p; E= -113.88; ZPVE= 2.62 eV; frequencies= 3070.71, 3048.17, 3023.42, 3012.73, 2999.72, 2941.62, 2926.45, 1475.37, 1457.20, 1451.94, 1444.42, 1381.47, 1366.88, 1329.71, 1175.60, 1092.90, 1090.90, 1043.20, 1003.18, 918.97, 908.47, 899.99, 857.87, 537.83, 423.63, $387.69,245.06,223.41,207.73,197.23,70.81,56.99,17.61 \mathrm{~cm}^{\wedge}-1$

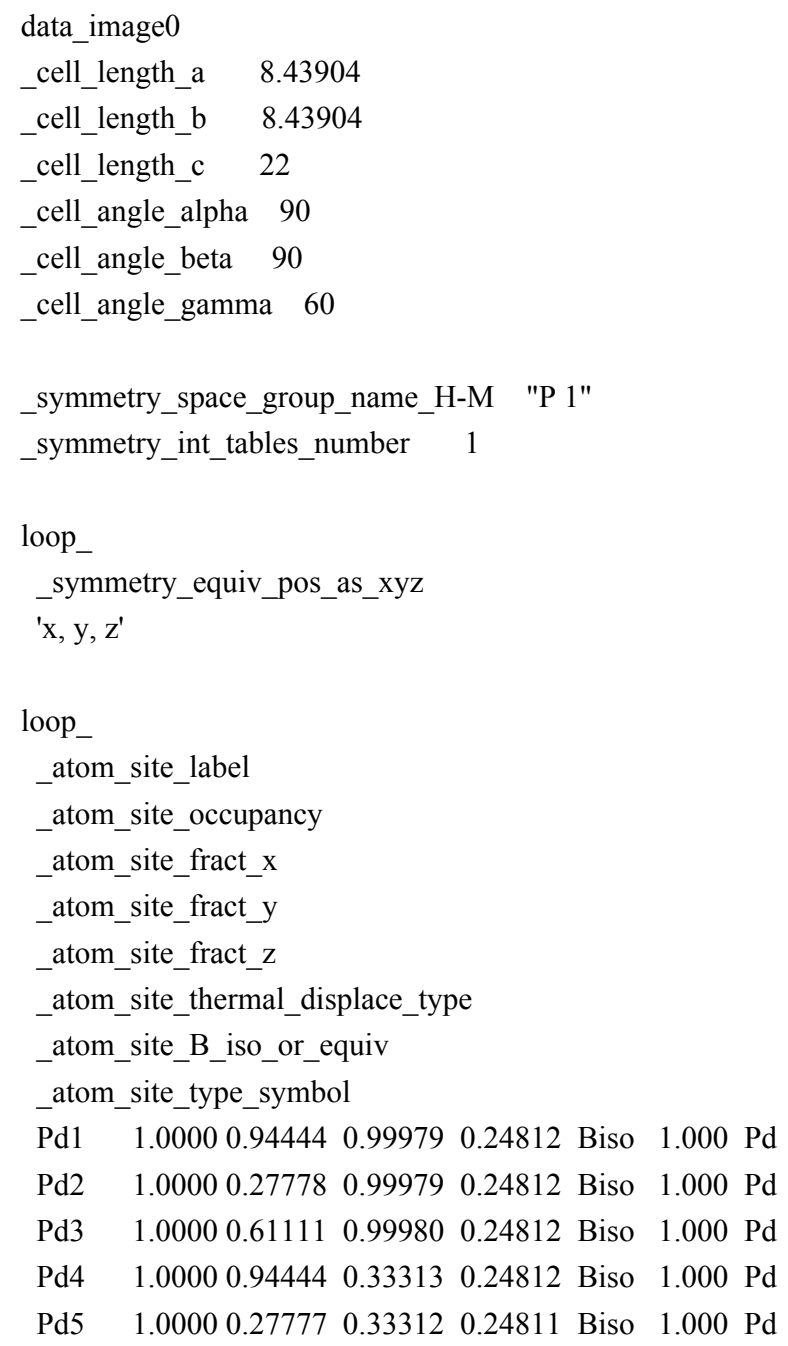




\begin{tabular}{|c|c|c|c|}
\hline Pd6 & 1.00000 .611120 .33312 & 0.24811 Biso & \\
\hline $\operatorname{Pd} 7$ & 1.00000 .944440 .66646 & 0.24812 Biso & 1.000 \\
\hline $\mathrm{Pd} 8$ & $1.0000 \quad 0.277770 .66647$ & 0.24811 Biso & 1.000 \\
\hline Pd9 & 1.00000 .611110 .66646 & 0.24812 Biso & 1.000 \\
\hline $\mathrm{d} 10$ & 1.00000 .055560 .11091 & 0.35314 Biso & 1.000 \\
\hline $\operatorname{Pd} 11$ & $\begin{array}{lll}1.0000 & 0.38889 & 0.11091\end{array}$ & 0.35315 Biso & 1.000 \\
\hline Pd12 & 1.00000 .722220 .11091 & 0.35315 Biso & 1.00 \\
\hline Pd13 & $\begin{array}{lll}1.0000 & 0.05556 & 0.44424\end{array}$ & 0.35315 Biso & 1.000 \\
\hline Pd14 & $\begin{array}{lll}1.0000 & 0.38889 & 0.44424\end{array}$ & 0.35313 Biso & 1.0 \\
\hline Pd15 & $\begin{array}{lll}1.0000 & 0.72222 & 0.44424\end{array}$ & 0.35315 Biso & 1.000 \\
\hline Pd16 & $\begin{array}{lll}1.0000 & 0.05556 & 0.77757\end{array}$ & 0.35315 Biso & 1.00 \\
\hline d17 & $\begin{array}{lll}1.0000 & 0.38889 & 0.77757\end{array}$ & 0.35315 Biso & 1.00 \\
\hline d18 & 1.00000 .722220 .77757 & 0.35314 Biso & 1.00 \\
\hline Pd19 & $\begin{array}{lll}1.0000 & 0.83295 & 0.22251\end{array}$ & 0.45675 Biso & 1.00 \\
\hline $\operatorname{Pd} 20$ & $\begin{array}{lll}1.0000 & 0.16730 & 0.22337\end{array}$ & 0.45943 Biso & 1.00 \\
\hline $\operatorname{Pd} 21$ & $\begin{array}{lll}1.0000 & 0.49638 & 0.22394\end{array}$ & 0.45900 Biso & 1.00 \\
\hline $\operatorname{Pd} 22$ & $\begin{array}{lll}1.0000 & 0.83301 & 0.55557\end{array}$ & 0.45676 Biso & 1.00 \\
\hline $\operatorname{Pd} 23$ & 1.00000 .167590 .55262 & 0.45866 Biso & 1.000 \\
\hline $\mathrm{Pd} 24$ & $\begin{array}{lll}1.0000 & 0.49991 & 0.55552\end{array}$ & 0.45634 Biso & 1.000 \\
\hline $\operatorname{Pd} 25$ & 1.00000 .833120 .88901 & 0.45638 Biso & 1.00 \\
\hline $\operatorname{Pd} 26$ & 1.00000 .166490 .88903 & 0.45650 Biso & 1.00 \\
\hline $\operatorname{Pd} 27$ & 1.00000 .499910 .88868 & 0.45663 Biso & 1.00 \\
\hline $\operatorname{Pd} 28$ & $\begin{array}{lll}1.0000 & 0.94331 & 0.00023\end{array}$ & 0.56141 Biso & 1.000 \\
\hline $\operatorname{Pd} 29$ & $1.0000 \quad 0.280100 .99736$ & 0.56285 Biso & 1.000 \\
\hline $\operatorname{Pd} 30$ & $\begin{array}{lll}1.0000 & 0.61184 & 0.00021\end{array}$ & 0.56121 Biso & 1.000 \\
\hline $\mathrm{Pd} 31$ & $1.00000 .94090 \quad 0.33644$ & 0.56337 Biso & 1.000 \\
\hline $\operatorname{Pd} 32$ & $\begin{array}{lll}1.0000 & 0.28012 & 0.33920\end{array}$ & 0.57883 Biso & 1.00 \\
\hline $\operatorname{Pd} 33$ & $\begin{array}{lll}1.0000 & 0.61097 & 0.33381\end{array}$ & 0.56137 Biso & 1.00 \\
\hline $\mathrm{Pd} 34$ & 1.00000 .943120 .66860 & 0.56151 Biso & 1.00 \\
\hline $\mathrm{Pd} 35$ & $\begin{array}{lll}1.0000 & 0.27726 & 0.66742\end{array}$ & 0.56048 Biso & 1.000 \\
\hline $\operatorname{Pd} 36$ & 1.00000 .611120 .66723 & 0.56130 Biso & 1.000 \\
\hline $\mathrm{C} 1$ & $\begin{array}{lll}1.0000 & 0.28007 & 0.36412\end{array}$ & 0.67487 Biso & 1.000 \\
\hline $\mathrm{C} 2$ & $1.00000 .30294 \quad 0.52324$ & 0.69492 Biso & 1.000 \\
\hline $\mathrm{C} 3$ & 1.00000 .420990 .18244 & 0.70199 Biso & 1.000 \\
\hline H1 & $\begin{array}{lll}1.0000 & 0.15521 & 0.21009\end{array}$ & 0.59912 Biso & 1.000 \\
\hline $\mathrm{H} 2$ & $\begin{array}{lll}1.0000 & 0.14053 & 0.39251\end{array}$ & 0.68338 Biso & 1.000 \\
\hline $\mathrm{H} 3$ & 1.00000 .199750 .65371 & 0.67529 Biso & 1.000 \\
\hline $\mathrm{H} 4$ & 1.00000 .399740 .06990 & 0.68863 Biso & 1.000 \\
\hline H5 & $1.0000 \quad 0.28540 \quad 0.53700$ & 0.74466 Biso & 1.000 \\
\hline H6 & $\begin{array}{lll}1.0000 & 0.43921 & 0.50167\end{array}$ & 0.68474 Biso & 1.000 \\
\hline H7 & $\begin{array}{lll}1.0000 & 0.56080 & 0.14713\end{array}$ & 0.69015 Biso & 1.000 \\
\hline H8 & $\begin{array}{lll}1.0000 & 0.40980 & 0.19411\end{array}$ & 0.75120 Biso & 1.000 \\
\hline
\end{tabular}

\# Pd1.B1.T2; E= -113.09; ZPVE= 2.44 eV; frequencies=1037.14i, 3101.52, 3080.05, 3065.44, 3044.01, 3012.26, 2991.57, 1497.19, $1464.09,1449.73,1400.53,1371.79,1338.61,1163.84,1104.36,1053.20,1051.80,1013.63,963.97,903.42,899.98,878.29,726.64,568.49$, $539.85,422.07,380.09,292.97,187.47,142.35,104.03,82.86,61.47 \mathrm{~cm}^{\wedge}-1$

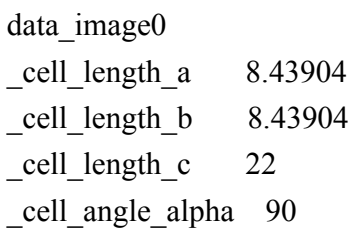




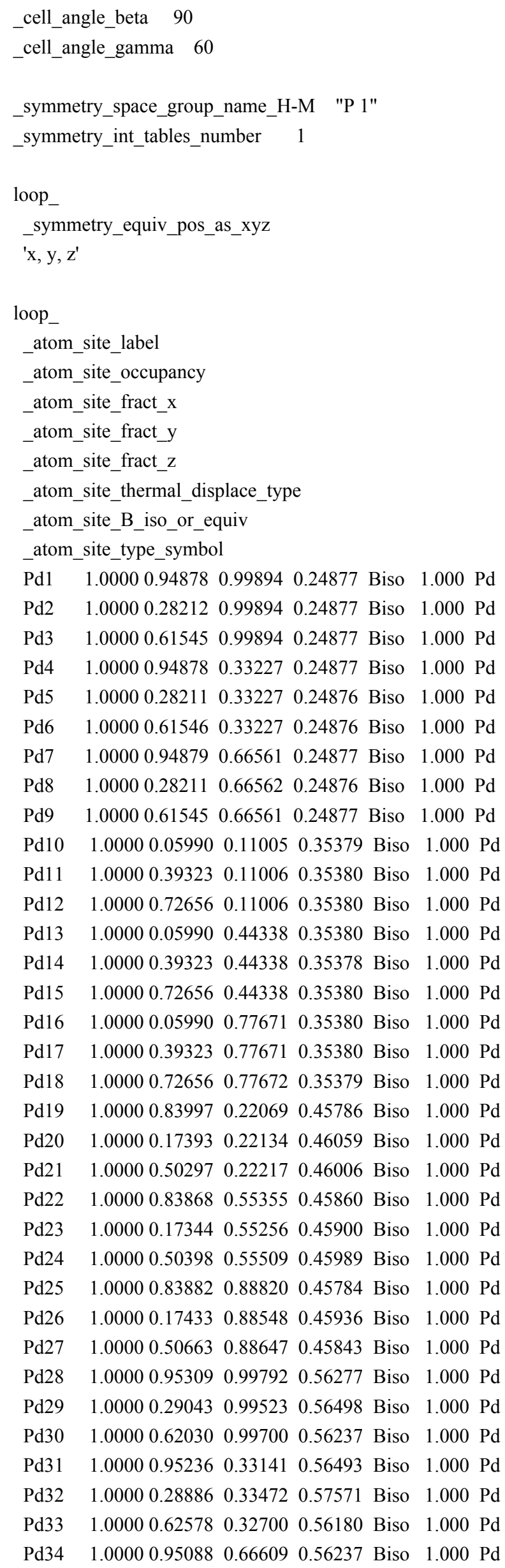




$\begin{array}{llllllll}\text { Pd35 } & 1.0000 & 0.28959 & 0.65920 & 0.56711 & \text { Biso } & 1.000 \mathrm{Pd} \\ \mathrm{Pd} 36 & 1.0000 & 0.62145 & 0.66382 & 0.56327 & \text { Biso } & 1.000 \mathrm{Pd} \\ \mathrm{C} 1 & 1.0000 & 0.27575 & 0.41767 & 0.66829 & \text { Biso } & 1.000 \mathrm{C} \\ \mathrm{C} 2 & 1.0000 & 0.24208 & 0.60695 & 0.66615 & \text { Biso } & 1.000 \mathrm{C} \\ \mathrm{C} 3 & 1.0000 & 0.44847 & 0.27826 & 0.70076 & \text { Biso } & 1.000 \mathrm{C} \\ \mathrm{H} 1 & 1.0000 & 0.16760 & 0.20682 & 0.60261 & \text { Biso } & 1.000 \mathrm{H} \\ \mathrm{H} 2 & 1.0000 & 0.15491 & 0.41188 & 0.68260 & \text { Biso } & 1.000 \mathrm{H} \\ \mathrm{H} 3 & 1.0000 & 0.10999 & 0.71530 & 0.61440 & \text { Biso } & 1.000 \mathrm{H} \\ \mathrm{H} 4 & 1.0000 & 0.47175 & 0.13947 & 0.69582 & \text { Biso } & 1.000 \mathrm{H} \\ \mathrm{H} 5 & 1.0000 & 0.12433 & 0.70548 & 0.69269 & \text { Biso } & 1.000 \mathrm{H} \\ \mathrm{H} 6 & 1.0000 & 0.36032 & 0.62344 & 0.67524 & \text { Biso } & 1.000 \mathrm{H} \\ \mathrm{H} 7 & 1.0000 & 0.56987 & 0.28045 & 0.68444 & \text { Biso } & 1.000 \mathrm{H} \\ \mathrm{H} 8 & 1.0000 & 0.43420 & 0.31082 & 0.74823 & \text { Biso } & 1.000 \mathrm{H}\end{array}$

\# Pd1.B1.y; $E=-113.85 ; Z P V E=2.49$ eV; frequencies=3157.00, 3094.20, 3076.04, 3066.77, 3043.24, 2973.79, 1518.14, 1471.38, 1454.54, 1403.63, 1383.26, 1253.94, 1168.59, 1049.68, 1032.39, 974.94, 962.92, 950.38, 918.68, 897.16, 881.80, 835.81, 750.76, 687.64, 663.16, $411.42,336.43,212.74,206.70,143.20,68.88,53.42,34.81 \mathrm{~cm}^{\wedge}-1$

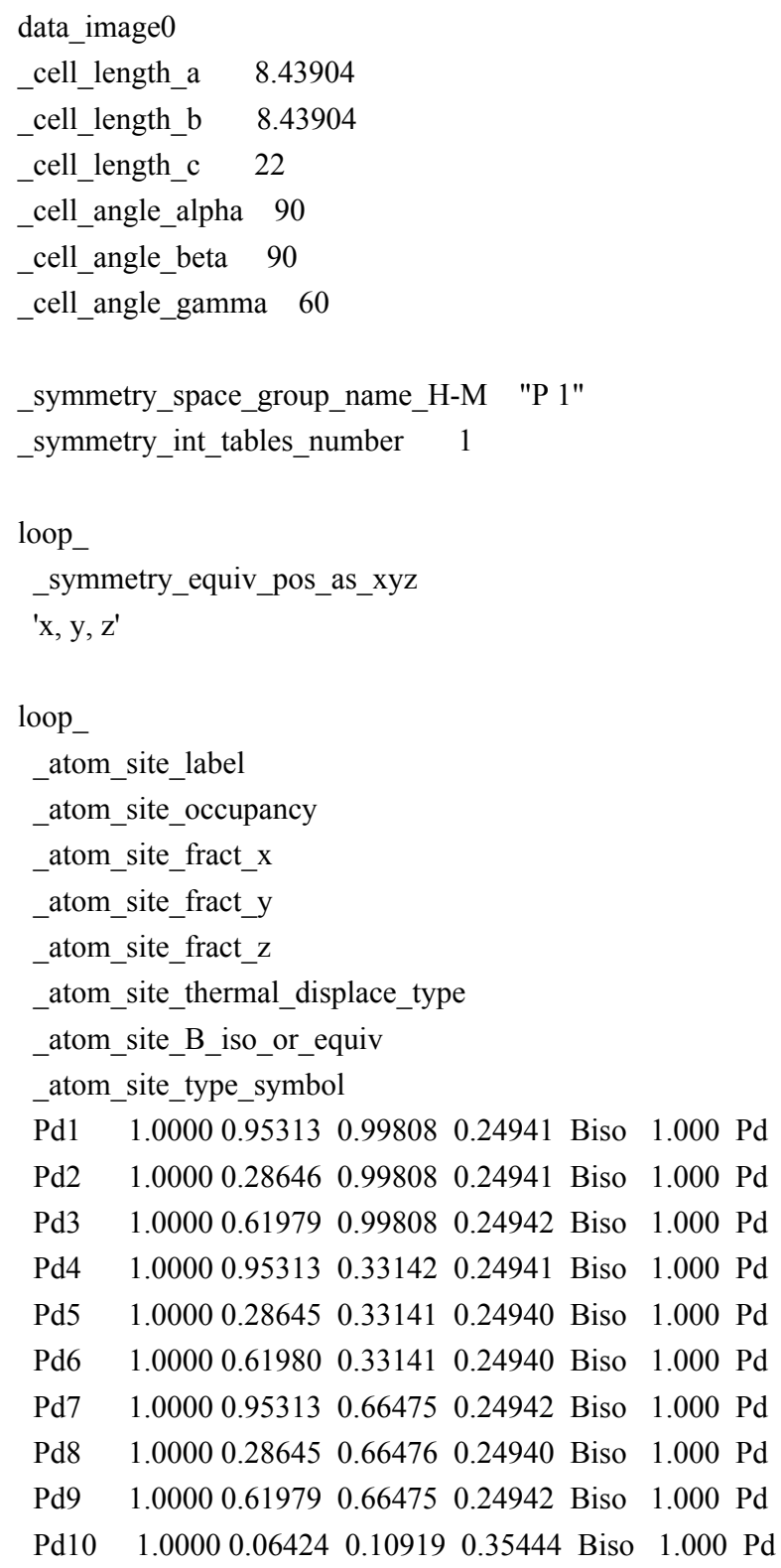




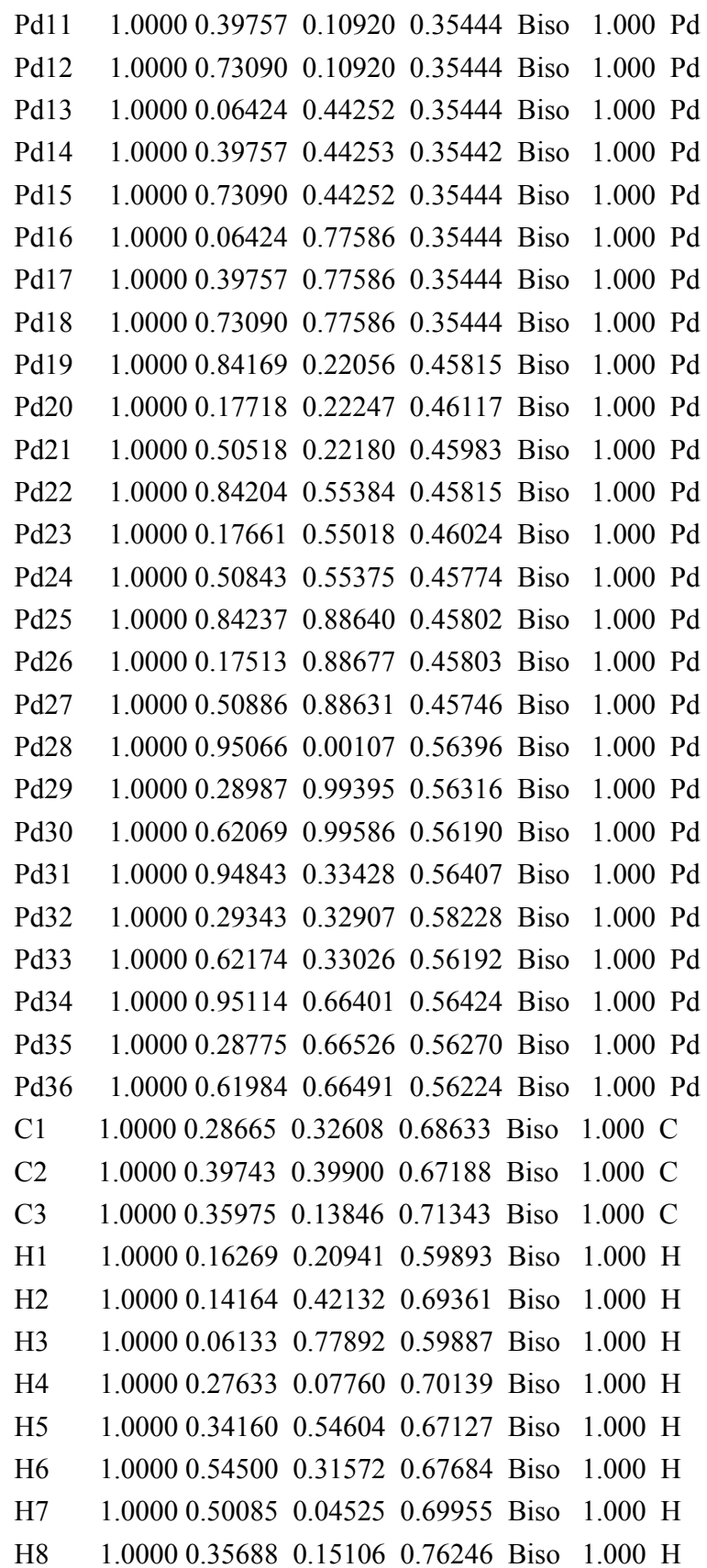

\# Pd2.A1.P; $E=-113.96 ; Z P V E=2.77 \mathrm{eV}$; frequencies $=15.23 \mathrm{i}, 3053.21,3047.70,3039.75,3033.72,2984.40,2975.14,2969.36,2942.02$, 1491.94, 1486.93, 1477.94, 1468.50, 1463.92, 1401.87, 1381.96, 1340.73, 1295.55, 1192.29, 1155.98, 1043.75, 919.51, 900.28, 857.08, $743.88,369.21,271.63,223.08,55.96,53.10,40.10,38.46,23.74 \mathrm{~cm}^{\wedge}-1$

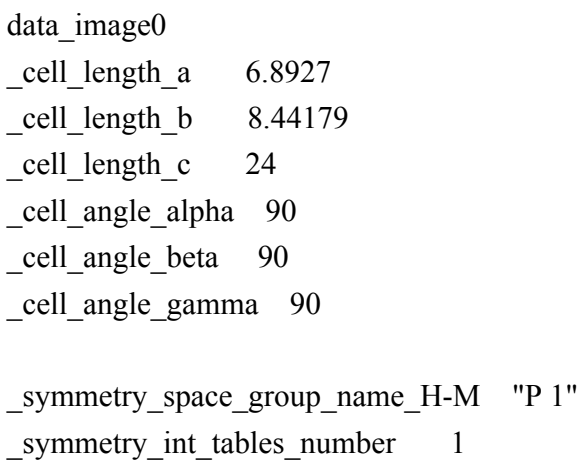




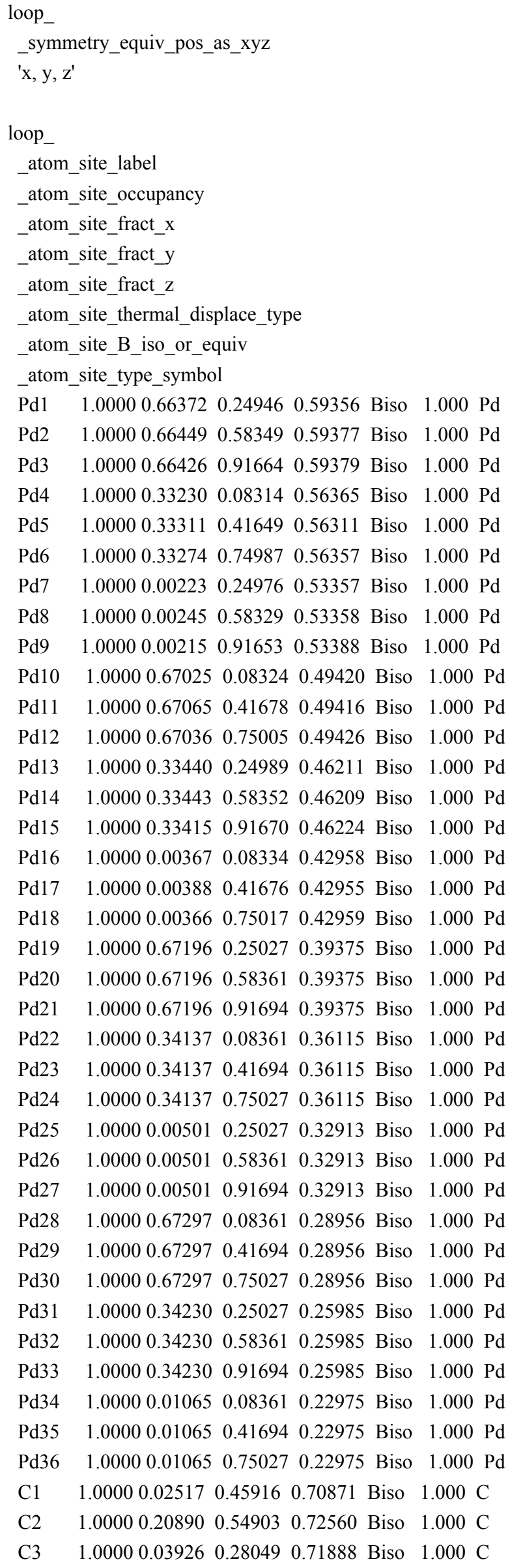




$\begin{array}{llllllll}\text { H1 } & 1.0000 & 0.99616 & 0.48053 & 0.66434 & \text { Biso } & 1.000 \mathrm{H} \\ \text { H2 } & 1.0000 & 0.90031 & 0.50722 & 0.73152 & \text { Biso } & 1.000 \mathrm{H} \\ \text { H3 } & 1.0000 & 0.19638 & 0.67561 & 0.71643 & \text { Biso } & 1.000 \mathrm{H} \\ \text { H4 } & 1.0000 & 0.90644 & 0.21941 & 0.70598 & \text { Biso } & 1.000 \mathrm{H} \\ \text { H5 } & 1.0000 & 0.23772 & 0.53615 & 0.77022 & \text { Biso } & 1.000 \mathrm{H} \\ \text { H6 } & 1.0000 & 0.33564 & 0.50390 & 0.70305 & \text { Biso } & 1.000 \mathrm{H} \\ \text { H7 } & 1.0000 & 0.15988 & 0.22871 & 0.69537 & \text { Biso } & 1.000 \mathrm{H} \\ \text { H8 } & 1.0000 & 0.06287 & 0.25465 & 0.76245 & \text { Biso } & 1.000 \mathrm{H}\end{array}$

\# Pd2.A1.T1; $E=-113.01 ; Z P V E=2.61 \mathrm{eV}$; frequencies $=674.28 \mathrm{i}, 3066.27,3059.92,3054.94,3025.89,2993.98,2987.99,2970.12,1490.11$, 1480.53, 1472.08, 1470.30, 1411.65, 1386.21, 1323.11, 1297.54, 1198.04, 1130.36, 1071.12, 996.61, 944.32, 883.09, 799.20, 704.81, 542.60, $415.87,255.81,239.34,181.32,130.26,53.37,45.19,39.58 \mathrm{~cm}^{\wedge}-1$

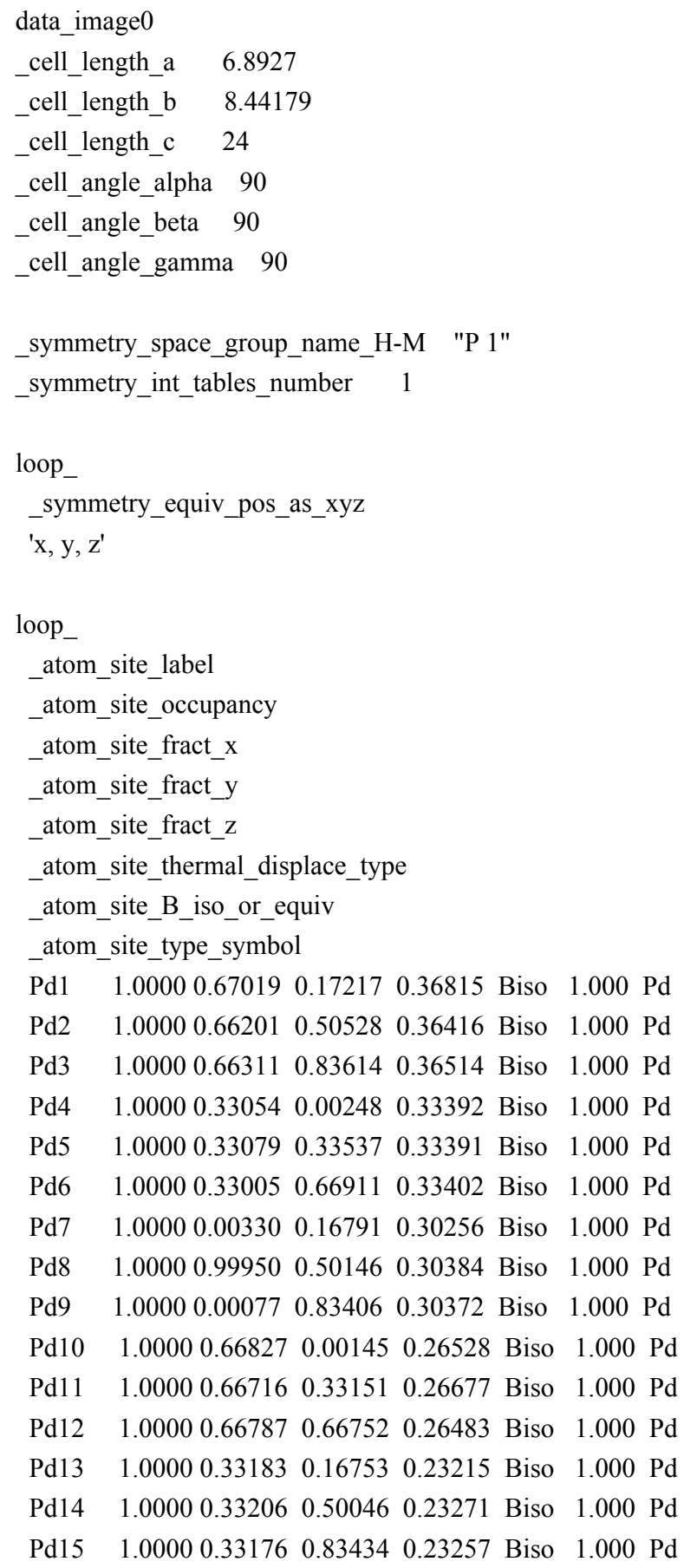




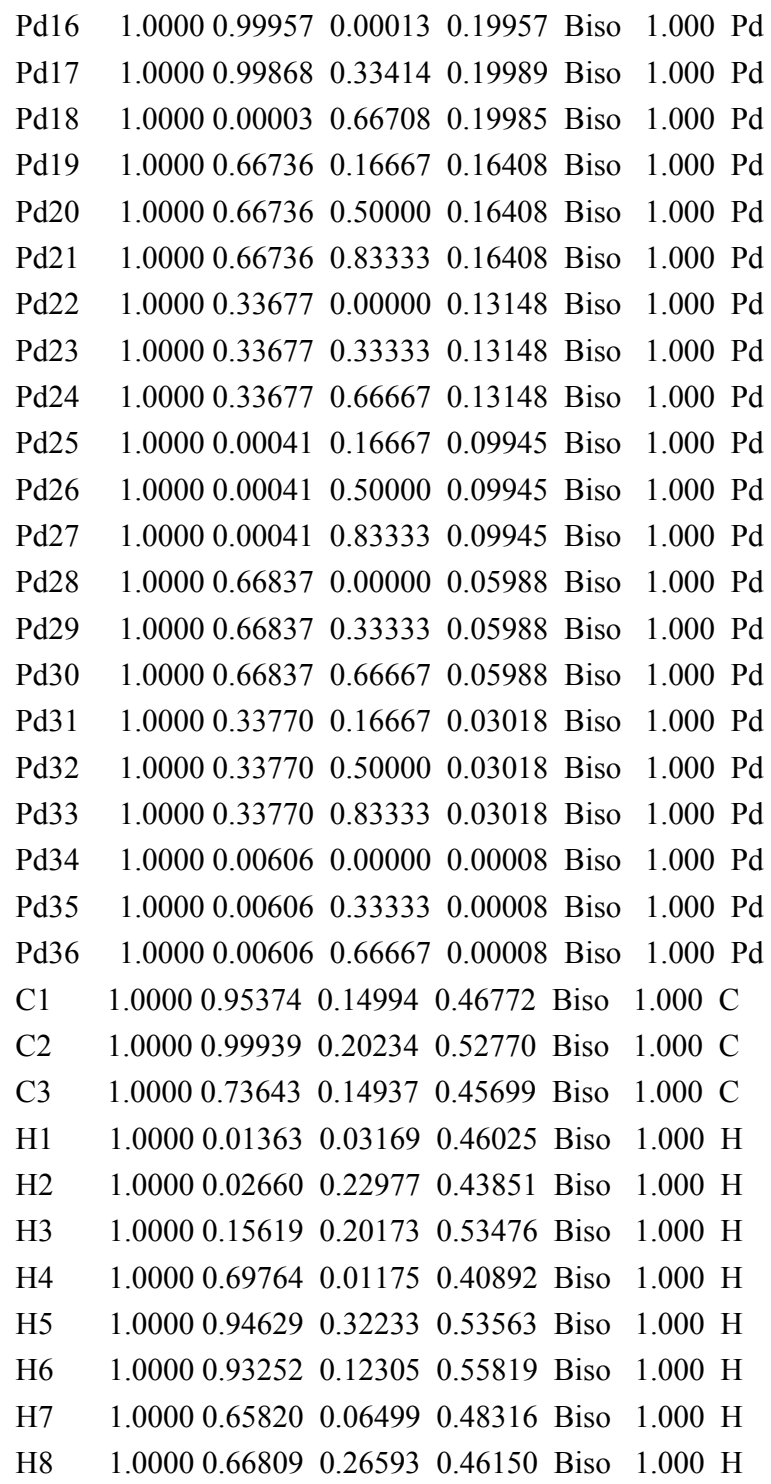

\# Pd2.A1.1p; $E=-113.47 ; \mathrm{ZPVE}=2.62 \mathrm{eV}$; frequencies=3065.62, 3060.77, 3035.30, 3013.12, 2987.66, 2982.37, 2965.49, 1489.54, 1479.19, $1468.75,1432.31,1382.09,1310.21,1295.52,1196.15,1102.88,1018.20,974.59,925.84,901.67,881.71,778.86,763.68,759.31,622.21$, $535.31,248.32,245.51,144.90,87.04,53.02,38.17,27.54 \mathrm{~cm}^{\wedge}-1$

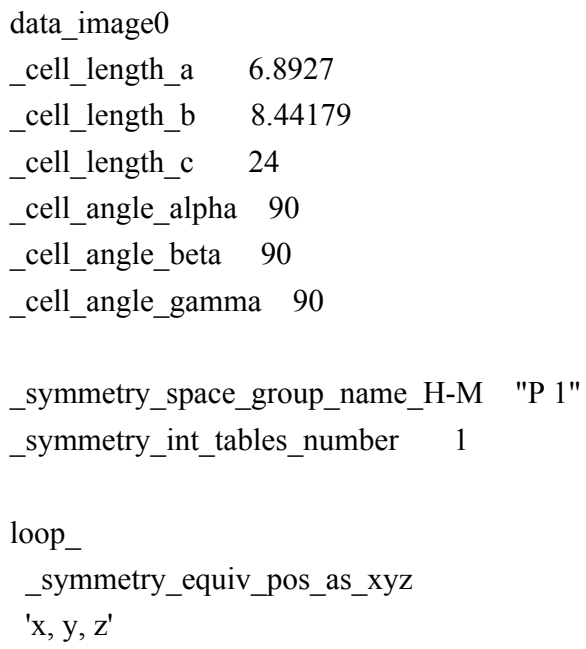




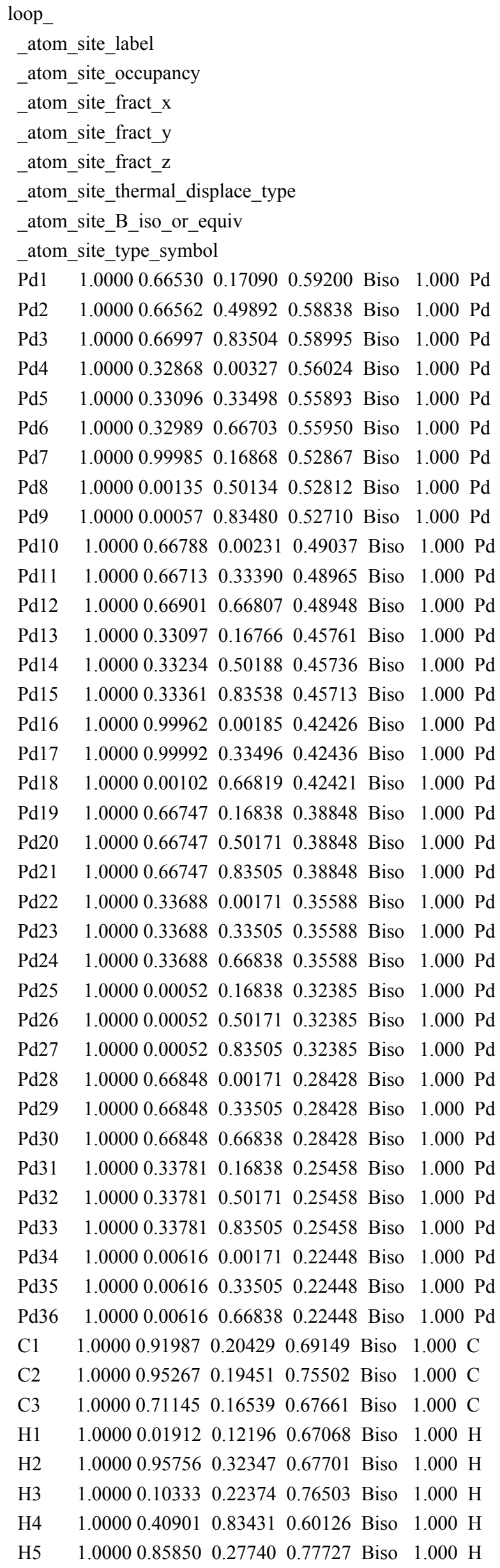


H6 $\quad 1.00000 .922760 .075220 .77058$ Biso $1.000 \mathrm{H}$

H7 1.00000 .668910 .045140 .68878 Biso $1.000 \mathrm{H}$

H8 1.00000 .606200 .251250 .69279 Biso $1.000 \mathrm{H}$

\# Pd2.A1.T2; E= -112.88; ZPVE=2.47 eV; frequencies=699.16i, 3163.60, 3090.78, 3071.06, 3067.35, 3061.21, 2993.08, 1486.28, 1479.41, 1464.28, 1395.06, 1377.24, 1279.37, 1207.44, 1152.81, 1092.82, 1029.31, 976.21, 951.42, 931.71, 902.49, 890.35, 731.69, 665.56, 630.48, $428.70,402.66,324.10,212.86,151.95,108.05,70.91,31.75 \mathrm{~cm}^{\wedge}-1$

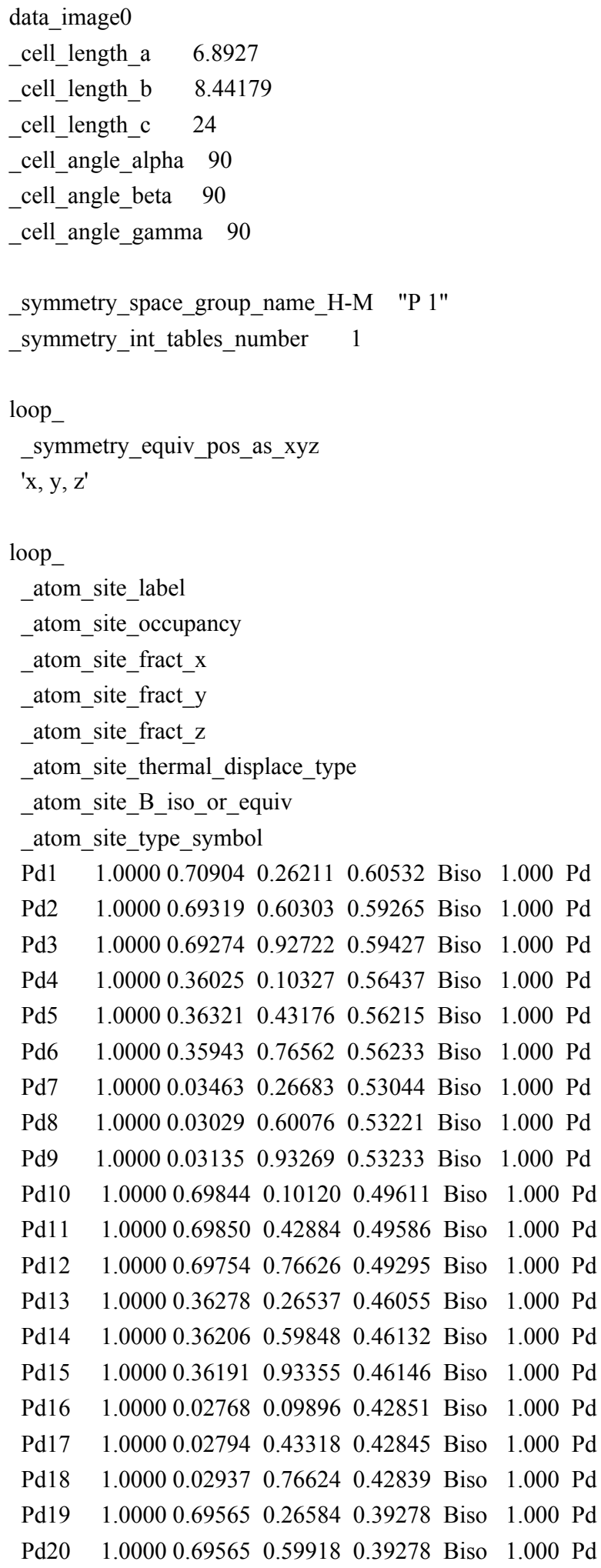




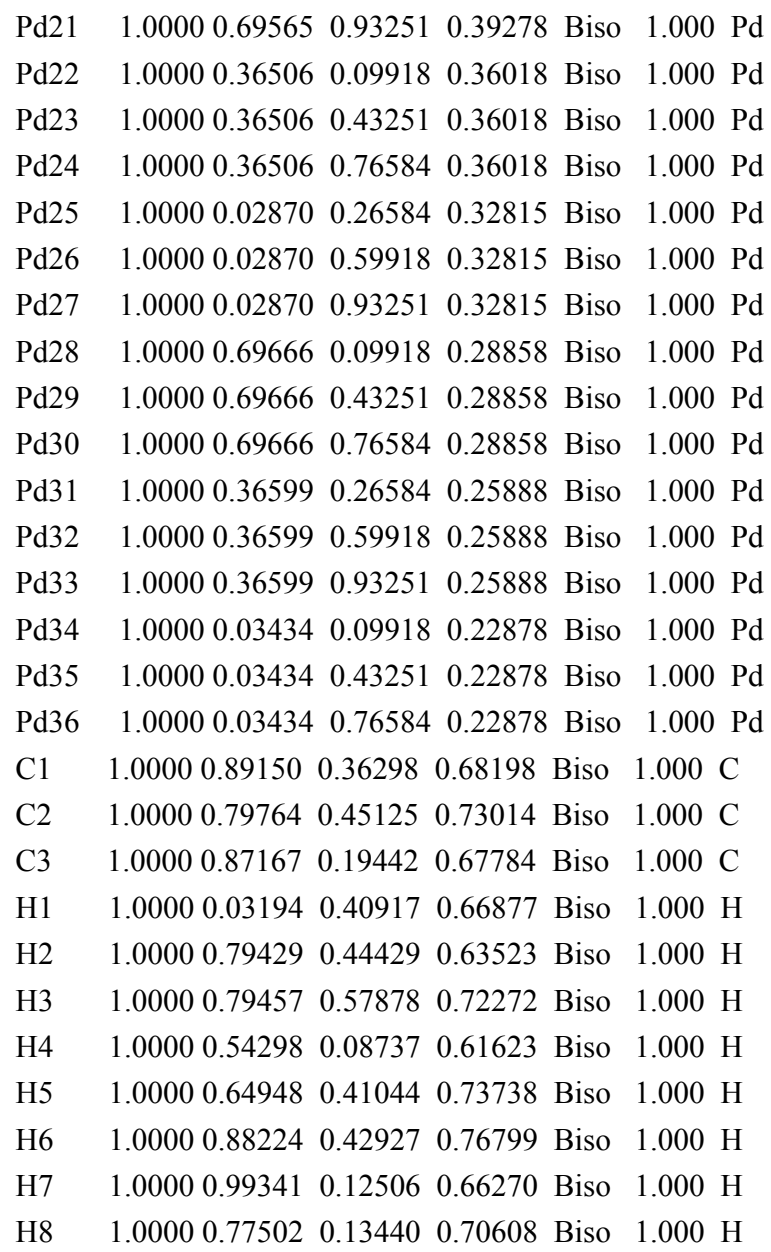

\# Pd2.A1.y; $E=-113.38 ; Z P V E=2.41 \mathrm{eV}$; frequencies=35.28i, 3161.43, 3089.26, 3072.77, 3065.76, 3025.10, 2960.04, 1536.19, 1471.98, $1456.93,1408.84,1382.45,1264.26,1167.47,1120.64,1025.72,969.45,918.15,913.58,884.36,832.50,719.40,681.38,628.46,535.51$, $404.02,335.25,234.32,210.03,193.46,140.05,69.79,54.74 \mathrm{~cm}^{\wedge}-1$

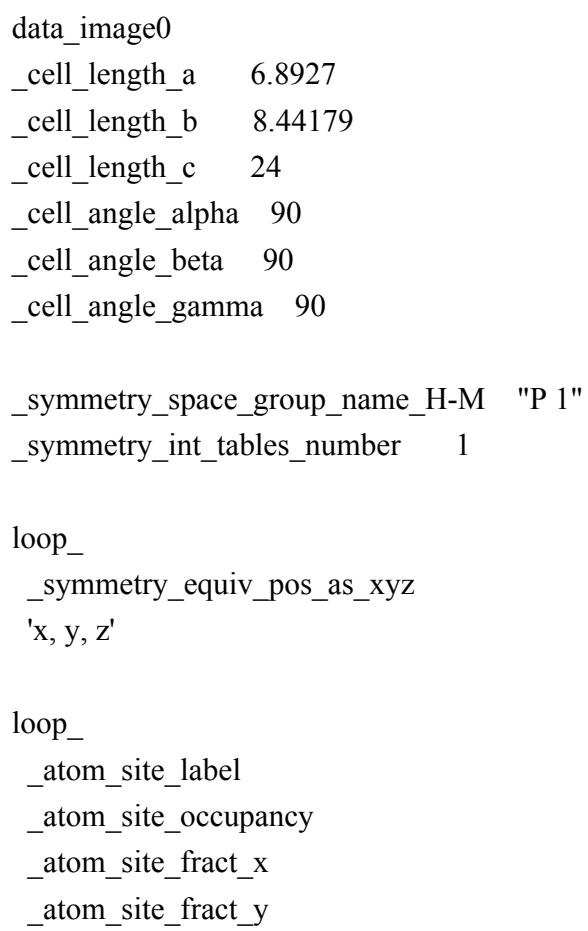




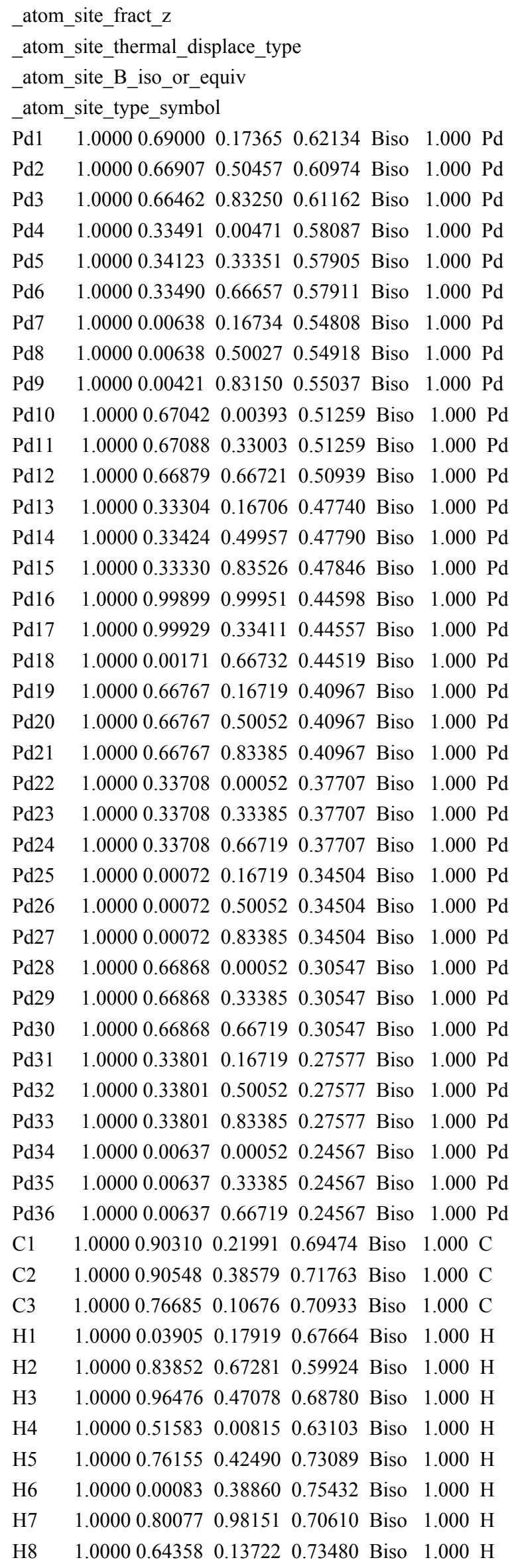


\# Pd2.B1.P; $E=-113.96 ; Z P V E=2.77$ eV; frequencies=41.23i, 3052.47, 3048.29, 3041.76, 3037.18, 2985.36, 2978.65, 2973.70, 2941.57, 1490.98, 1486.88, 1476.95, 1468.64, 1462.41, 1401.34, 1381.57, 1340.01, 1294.78, 1191.38, 1155.59, 1044.07, 918.61, 898.63, 857.30, $740.87,369.23,269.99,219.50,54.82,50.84,33.43,15.19,5.14 \mathrm{~cm}^{\wedge}-1$

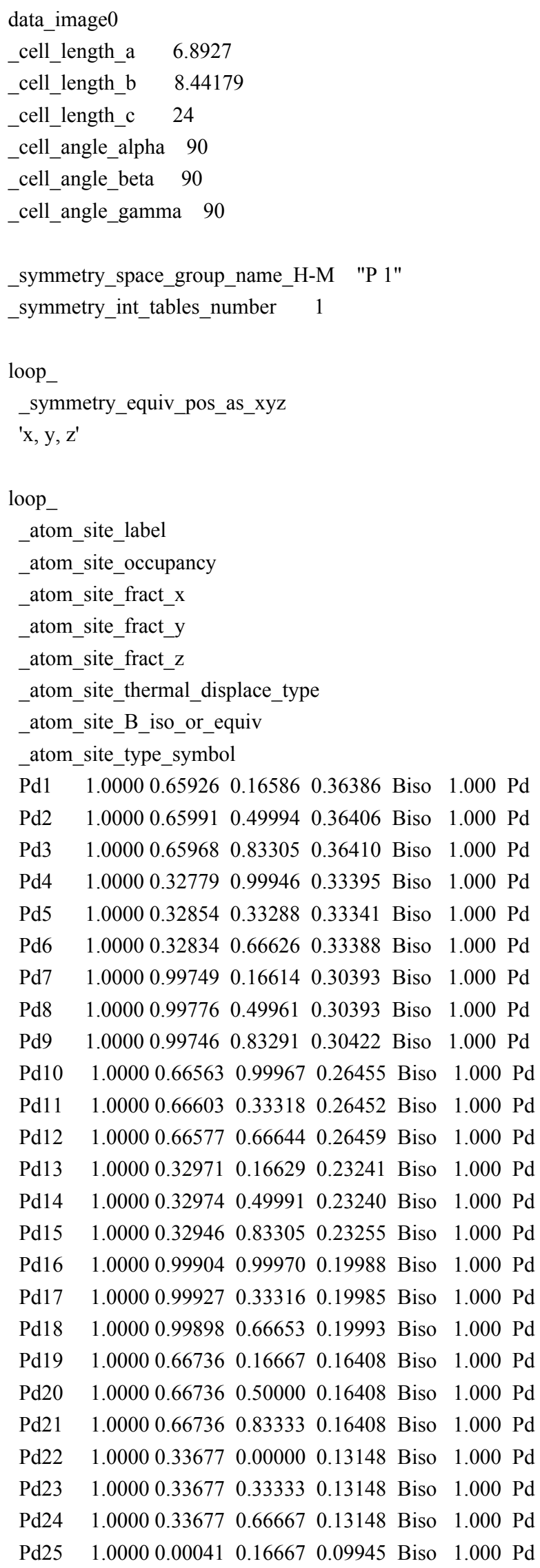




$\begin{array}{lllllll}\mathrm{Pd} 26 & 1.0000 & 0.00041 & 0.50000 & 0.09945 & \text { Biso } & 1.000 \mathrm{Pd} \\ \mathrm{Pd} 27 & 1.0000 & 0.00041 & 0.83333 & 0.09945 & \text { Biso } & 1.000 \mathrm{Pd} \\ \mathrm{Pd} 28 & 1.0000 & 0.66837 & 0.00000 & 0.05988 & \text { Biso } & 1.000 \mathrm{Pd} \\ \mathrm{Pd} 29 & 1.0000 & 0.66837 & 0.33333 & 0.05988 & \text { Biso } & 1.000 \mathrm{Pd} \\ \mathrm{Pd} 30 & 1.0000 & 0.66837 & 0.66667 & 0.05988 & \text { Biso } & 1.000 \mathrm{Pd} \\ \mathrm{Pd} 31 & 1.0000 & 0.33770 & 0.16667 & 0.03018 & \text { Biso } & 1.000 \mathrm{Pd} \\ \mathrm{Pd} 32 & 1.0000 & 0.33770 & 0.50000 & 0.03018 & \text { Biso } & 1.000 \mathrm{Pd} \\ \mathrm{Pd} 33 & 1.0000 & 0.33770 & 0.83333 & 0.03018 & \text { Biso } & 1.000 \mathrm{Pd} \\ \mathrm{Pd} 34 & 1.0000 & 0.00606 & 0.00000 & 0.00008 & \text { Biso } & 1.000 \mathrm{Pd} \\ \mathrm{Pd} 35 & 1.0000 & 0.00606 & 0.33333 & 0.00008 & \text { Biso } & 1.000 \mathrm{Pd} \\ \mathrm{Pd} 36 & 1.0000 & 0.00606 & 0.66667 & 0.00008 & \text { Biso } & 1.000 \mathrm{Pd} \\ \mathrm{C} 1 & 1.0000 & 0.02057 & 0.37553 & 0.47902 & \text { Biso } & 1.000 \mathrm{C} \\ \mathrm{C} 2 & 1.0000 & 0.20427 & 0.46545 & 0.49594 & \text { Biso } & 1.000 \mathrm{C} \\ \mathrm{C} 3 & 1.0000 & 0.03459 & 0.19695 & 0.48921 & \text { Biso } & 1.000 \mathrm{C} \\ \mathrm{H} 1 & 1.0000 & 0.99153 & 0.39685 & 0.43464 & \text { Biso } & 1.000 \mathrm{H} \\ \mathrm{H} 2 & 1.0000 & 0.89574 & 0.42348 & 0.50184 & \text { Biso } & 1.000 \mathrm{H} \\ \mathrm{H} 3 & 1.0000 & 0.19182 & 0.59204 & 0.48674 & \text { Biso } & 1.000 \mathrm{H} \\ \mathrm{H} 4 & 1.0000 & 0.90190 & 0.13585 & 0.47630 & \text { Biso } & 1.000 \mathrm{H} \\ \mathrm{H} 5 & 1.0000 & 0.23307 & 0.45253 & 0.54058 & \text { Biso } & 1.000 \mathrm{H} \\ \mathrm{H} 6 & 1.0000 & 0.33097 & 0.42024 & 0.47340 & \text { Biso } & 1.000 \mathrm{H} \\ \mathrm{H} 7 & 1.0000 & 0.15519 & 0.14517 & 0.46575 & \text { Biso } & 1.000 \mathrm{H} \\ \mathrm{H} 8 & 1.0000 & 0.05814 & 0.17099 & 0.53276 & \text { Biso } & 1.000 \mathrm{H}\end{array}$

\# Pd2.B1.T1; E= -112.94; ZPVE= 2.58 eV; frequencies=44.56i, 812.92i, 3122.05, 3043.99, 3038.76, 3013.50, 2974.35, 2952.29, 2934.47, $1485.96,1468.14,1455.39,1450.37,1442.55,1388.45,1368.64,1295.44,1170.58,1101.87,1093.25,927.45,911.67,905.96,852.73$, $606.98,417.95,386.39,226.88,215.06,162.41,103.81,65.07,40.23 \mathrm{~cm}^{\wedge}-1$

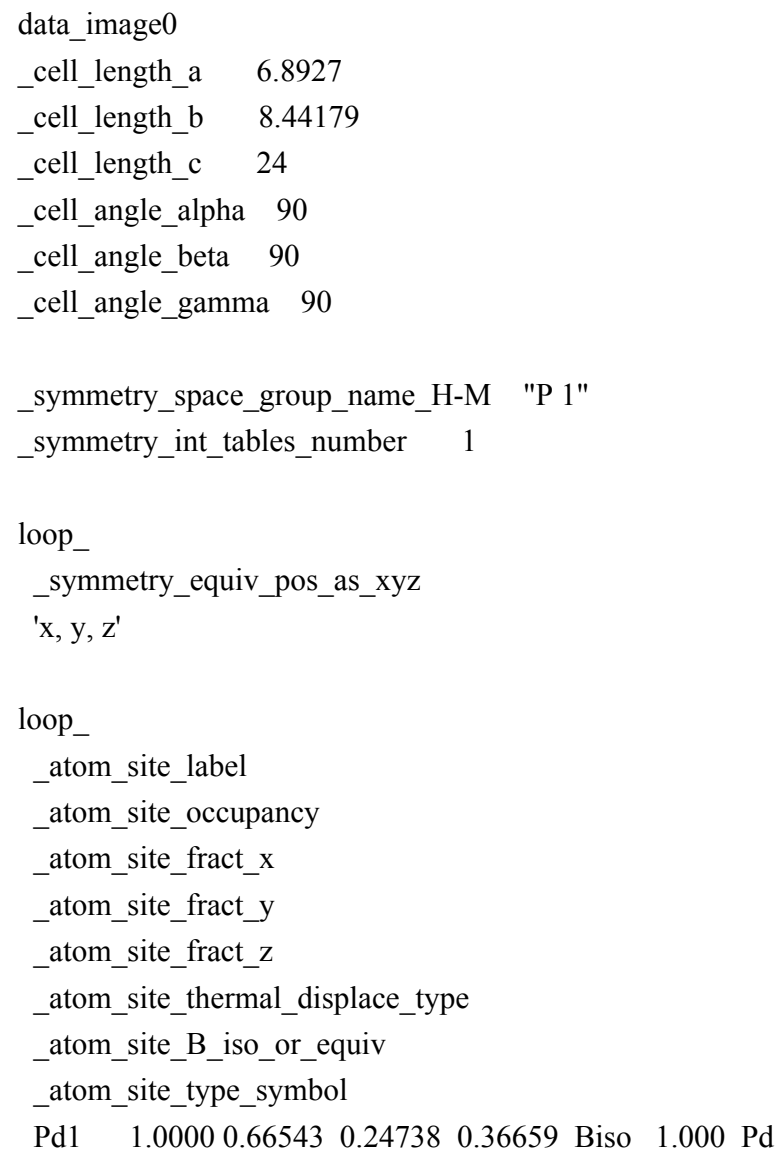




\begin{tabular}{|c|c|c|c|c|}
\hline $\mathrm{Pd} 2$ & 1.00000 .67412 & 0.58309 & 0.37433 Biso & 1.000 \\
\hline $\mathrm{Pd} 3$ & 1.00000 .66547 & 0.91860 & 0.36659 Biso & 1.000 \\
\hline $\mathrm{d} 4$ & 1.00000 .33341 & 0.08299 & 0.33609 Biso & 1.000 \\
\hline $\mathrm{d} 5$ & 1.00000 .33870 & 0.41791 & 0.33627 Biso & 1.000 \\
\hline$d 6$ & 1.00000 .33877 & 0.74805 & 0.33624 Biso & 1.000 \\
\hline $\operatorname{Pd} 7$ & 1.00000 .00238 & 0.24858 & 0.30549 Biso & 1.000 \\
\hline 18 & 1.00000 .00535 & 0.58295 & 0.30791 Biso & 1.000 \\
\hline Pd9 & 1.00000. & 0.91739 & 0.30549 Biso & 1.000 \\
\hline $\mathrm{d} 10$ & 1.00000 .67039 & 0.08301 & 0.26673 Biso & 1.00 \\
\hline Pd11 & 1.00000 .67045 & 0.41827 & 0.26854 Biso & 1.000 \\
\hline d12 & 1.00000 .67050 & 0.74774 & 0.26853 Biso & 1.000 \\
\hline$d 13$ & 1.00000 .33545 & 0.25026 & 0.23498 Biso & 1.00 \\
\hline $\mathrm{d} 14$ & 1.00000 .33336 & 0.58299 & 0.23488 Biso & 1.00 \\
\hline Pd15 & 1.00000 .33546 & 0.91575 & 0.23498 Biso & 1.000 \\
\hline Pd16 & 1.00000 .00201 & 0.08300 & 0.20214 Biso & 1.00 \\
\hline $\operatorname{Pd} 17$ & 1.00000 .99983 & 0.41654 & 0.20260 Biso & 1.00 \\
\hline $\operatorname{Pd} 18$ & 1.00000 .99985 & 0.74947 & 0.20260 Biso & 1.000 \\
\hline$d 19$ & 1.00000 .66740 & 0.24968 & 0.16583 Biso & 1.00 \\
\hline $\mathrm{d} 20$ & 1.00000 .66740 & 0.58302 & 0.16583 Biso & 1.000 \\
\hline $\mathrm{d} 21$ & 1.00000 .66740 & 0.91635 & 0.16583 Biso & 1.000 \\
\hline $\operatorname{Pd} 22$ & 1.00000 .33681 & 0.08302 & 0.13323 Biso & 1.00 \\
\hline $\mathrm{Pd} 23$ & 1.00000 .33681 & 0.41635 & 0.13323 Biso & 1.000 \\
\hline $\operatorname{Pd} 24$ & 1.00000 .33681 & 0.74968 & 0.13323 Biso & 1.000 \\
\hline $\operatorname{Pd} 25$ & 1.00000 .00045 & 0.24968 & 0.10120 Biso & 1.000 \\
\hline $\operatorname{Pd} 26$ & 1.00000 .00045 & 0.58302 & 0.10120 Biso & 1.000 \\
\hline $\operatorname{Pd} 27$ & 1.00000 .00045 & 0.91635 & 0.10120 Biso & 1.000 \\
\hline $\mathrm{d} 28$ & 1.00000 .66841 & 0.08302 & 0.06163 Biso & 1.00 \\
\hline d29 & 1.00000 .66841 & 0.41635 & 0.06163 Biso & 1.00 \\
\hline $\operatorname{Pd} 30$ & 1.00000 .66841 & 0.74968 & 0.06163 Biso & 1.000 \\
\hline $\mathrm{d} 31$ & 1.00000 .33774 & 0.24968 & 0.03193 Biso & 1.000 \\
\hline $\operatorname{Pd} 32$ & 1.00000 .33774 & 0.58302 & 0.03193 Biso & 1.000 \\
\hline $\operatorname{Pd} 33$ & 1.00000 .33774 & 0.91635 & 0.03193 Biso & 1.000 \\
\hline $\operatorname{Pd} 34$ & 1.00000 .00609 & 0.08302 & 0.00183 Biso & 1.00 \\
\hline Pd35 & 1.00000 .00609 & 0.41635 & 0.00183 Biso & 1.00 \\
\hline $\operatorname{Pd} 36$ & 1.00000 .00609 & 0.74968 & 0.00183 Biso & 1.00 \\
\hline $\mathrm{C} 1$ & 1.00000 .86012 & & 0.44991 Biso & 1.000 \\
\hline $\mathrm{C} 2$ & 1.00000 .97121 & 0.73281 & 0.46463 Biso & 1.000 \\
\hline $\mathrm{C} 3$ & 1.00000 .96542 & 0.43085 & 0.46506 Biso & 1.000 \\
\hline H1 & 1.00000 .91542 & 0.58256 & 0.38229 Biso & 1.000 \\
\hline $\mathrm{H} 2$ & 1.00000 .71495 & 0.58520 & 0.46904 Biso & 1.000 \\
\hline H3 & 1.00000 .89264 & 0.84018 & 0.45260 Biso & 1.000 \\
\hline $\mathrm{H} 4$ & 1.00000 .88222 & 0.32523 & 0.45365 Biso & 1.000 \\
\hline H5 & 1.00000 .99295 & 0.73661 & 0.50997 Biso & 1.000 \\
\hline H6 & 1.00000 .11382 & 0.73624 & 0.44467 Biso & 1.000 \\
\hline H7 & 1.00000 .10733 & 0.42285 & 0.44487 Biso & 1.000 \\
\hline H8 & 1.00000 .98800 & 0.42793 & 0.50914 Biso & 1.000 \\
\hline
\end{tabular}

\# Pd2.B1.2p; E= -113.40; ZPVE= 2.60 eV; frequencies=16.47i, 3054.77, 3048.01, 2989.09, 2986.11, 2945.95, 2926.34, 2880.96, 1461.70, $1457.45,1446.50,1435.08,1380.28,1365.68,1321.29,1232.59,1166.29,1102.30,1089.52,1076.41,912.02,908.01,875.27,854.23$, $438.14,386.41,254.83,253.44,230.42,195.62,187.27,60.92,28.93 \mathrm{~cm}^{\wedge}-1$

data_image0 


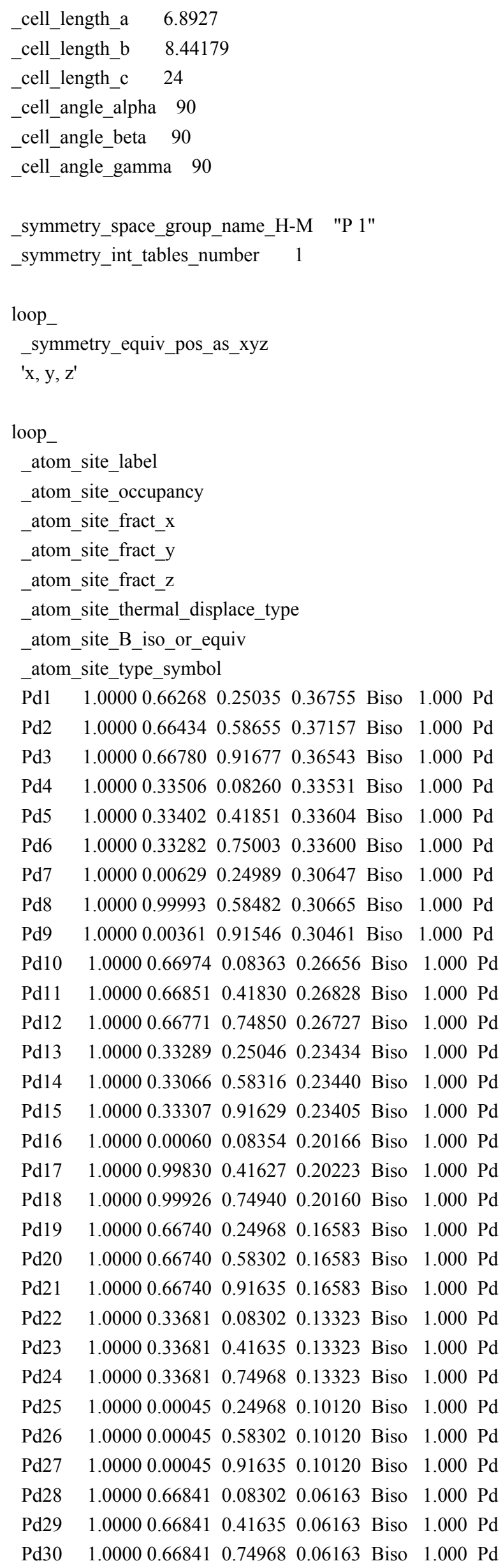




$\begin{array}{llllllll}\text { Pd31 } & 1.0000 & 0.33774 & 0.24968 & 0.03193 & \text { Biso } & 1.000 \mathrm{Pd} \\ \text { Pd32 } & 1.0000 & 0.33774 & 0.58302 & 0.03193 & \text { Biso } & 1.000 \mathrm{Pd} \\ \text { Pd33 } & 1.0000 & 0.33774 & 0.91635 & 0.03193 & \text { Biso } & 1.000 \mathrm{Pd} \\ \text { Pd34 } & 1.0000 & 0.00609 & 0.08302 & 0.00183 & \text { Biso } & 1.000 \mathrm{Pd} \\ \text { Pd35 } & 1.0000 & 0.00609 & 0.41635 & 0.00183 & \text { Biso } & 1.000 \mathrm{Pd} \\ \text { Pd36 } & 1.0000 & 0.00609 & 0.74968 & 0.00183 & \text { Biso } & 1.000 \mathrm{Pd} \\ \mathrm{C} 1 & 1.0000 & 0.67007 & 0.60303 & 0.45791 & \text { Biso } & 1.000 \mathrm{C} \\ \mathrm{C} 2 & 1.0000 & 0.77348 & 0.75333 & 0.47557 & \text { Biso } & 1.000 \mathrm{C} \\ \mathrm{C} 3 & 1.0000 & 0.76092 & 0.45422 & 0.48177 & \text { Biso } & 1.000 \mathrm{C} \\ \mathrm{H} 1 & 1.0000 & 0.91111 & 0.23233 & 0.37119 & \text { Biso } & 1.000 \mathrm{H} \\ \mathrm{H} 2 & 1.0000 & 0.51387 & 0.60903 & 0.46639 & \text { Biso } & 1.000 \mathrm{H} \\ \mathrm{H} 3 & 1.0000 & 0.70526 & 0.86112 & 0.45852 & \text { Biso } & 1.000 \mathrm{H} \\ \mathrm{H} 4 & 1.0000 & 0.68309 & 0.34663 & 0.46937 & \text { Biso } & 1.000 \mathrm{H} \\ \mathrm{H} 5 & 1.0000 & 0.76599 & 0.76563 & 0.52124 & \text { Biso } & 1.000 \mathrm{H} \\ \mathrm{H} 6 & 1.0000 & 0.92675 & 0.75269 & 0.46384 & \text { Biso } & 1.000 \mathrm{H} \\ \mathrm{H} 7 & 1.0000 & 0.91319 & 0.44191 & 0.46978 & \text { Biso } & 1.000 \mathrm{H} \\ \mathrm{H} 8 & 1.0000 & 0.75554 & 0.45976 & 0.52701 & \text { Biso } & 1.000 \mathrm{H}\end{array}$

\# Pd2.B1.T2; $E=-112.91 ; \mathrm{ZPVE}=2.45 \mathrm{eV}$; frequencies= 752.39i, 3152.89, 3076.33, 3060.84, 3057.61, 3034.49, 2954.37, 1476.69, 1466.43, $1450.49,1391.63,1375.91,1313.74,1211.04,1208.62,1160.75,1085.13,1045.43,1034.73,1014.48,905.97,876.25,834.22,448.64$, $404.07,394.75,308.86,272.30,189.13,178.56,108.87,55.53,37.23 \mathrm{~cm}^{\wedge}-1$

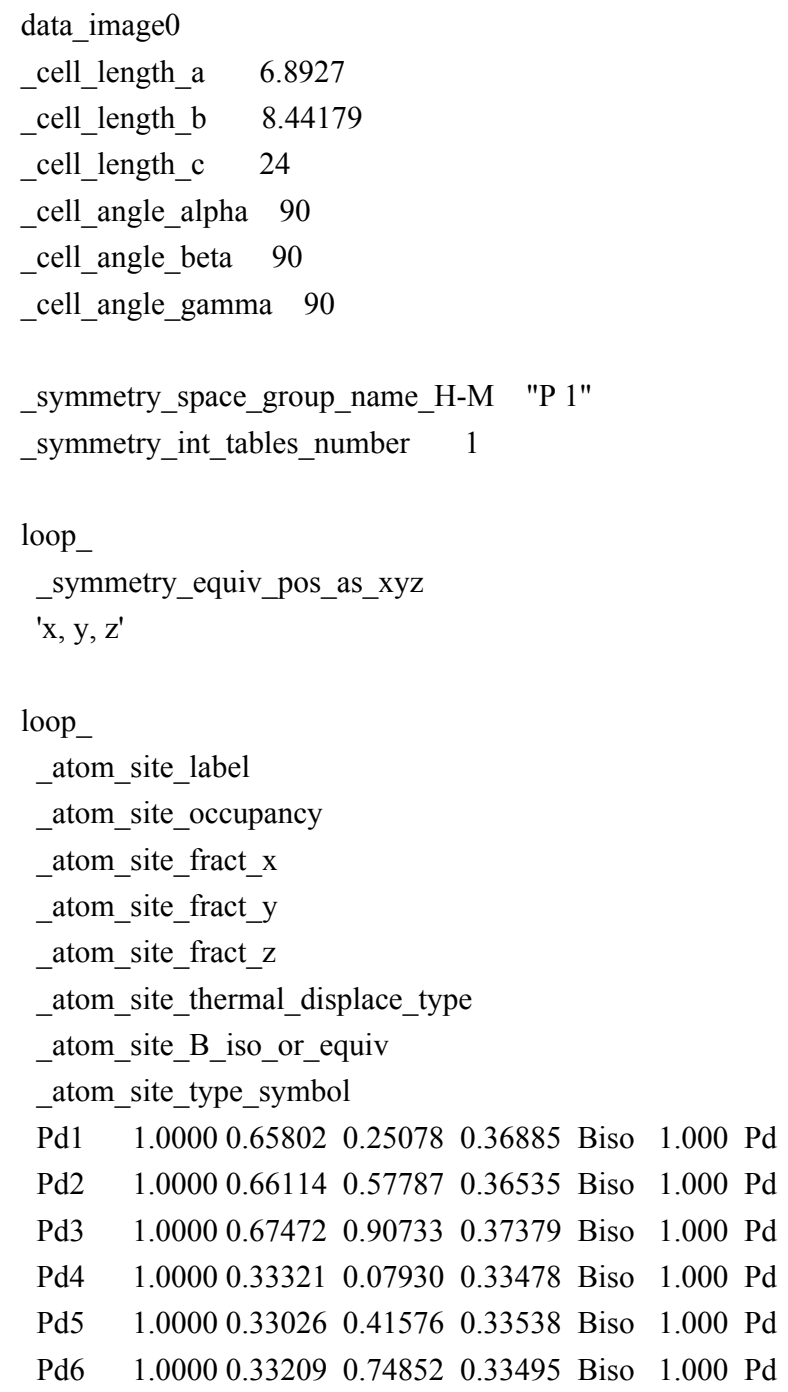




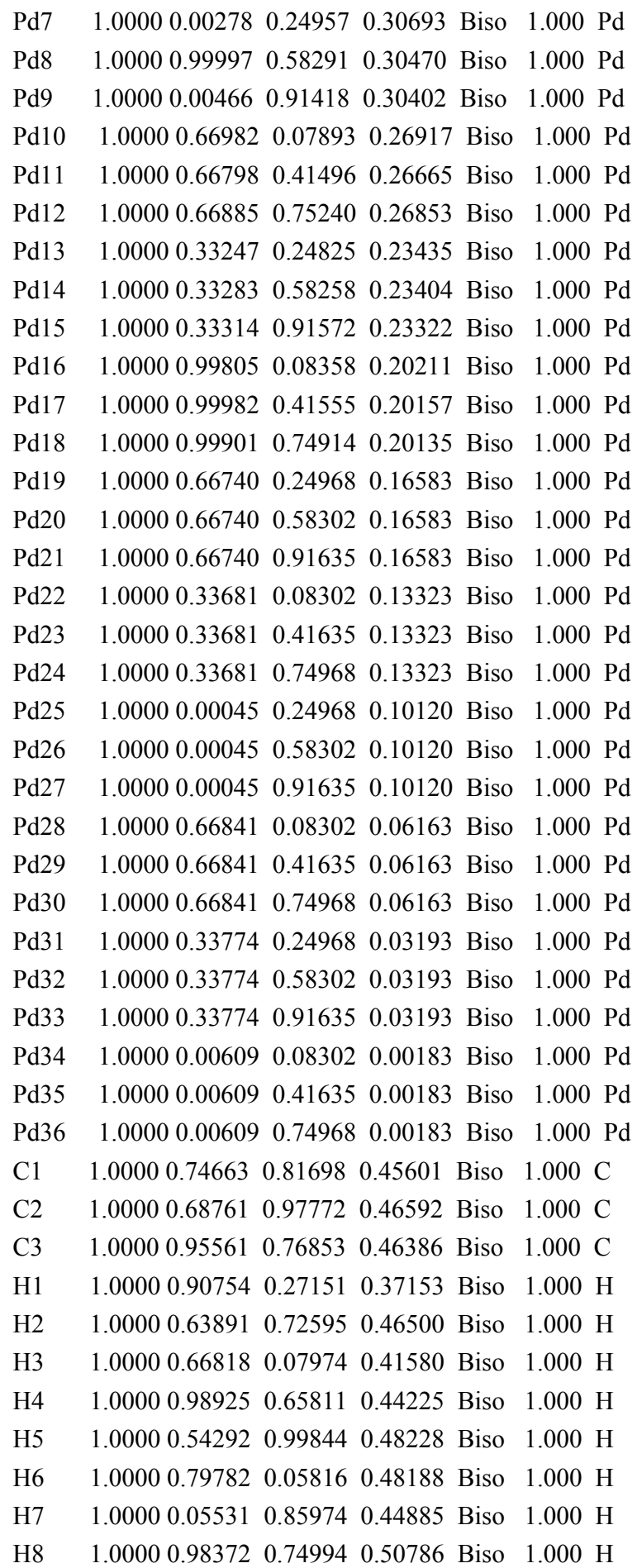

\# Pd2.B1.y; $\mathrm{E}=-113.46 ; \mathrm{ZPVE}=2.48 \mathrm{eV}$; frequencies= 20.57i, 3178.63, 3088.38, 3073.56, 3023.53, 3014.75, 2960.99, 1530.71, 1468.97, $1452.85,1404.35,1385.07,1246.61,1234.21,1169.62,1096.42,1044.86,1030.98,962.12,922.02,907.53,888.74,827.52,687.73,678.41$, $402.73,345.68,250.38,224.61,185.19,146.30,79.29,62.21 \mathrm{~cm}^{\wedge}-1$

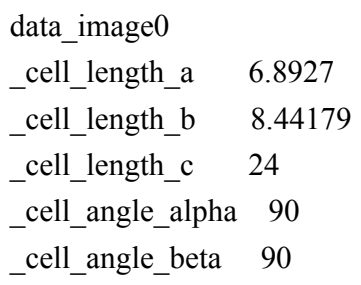




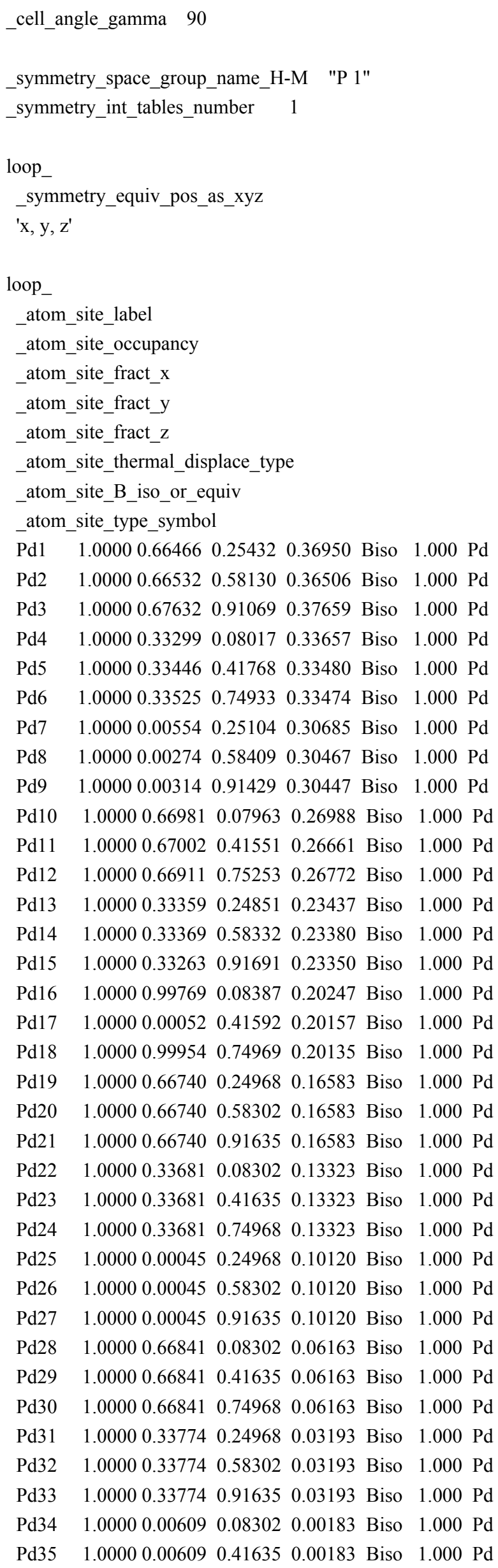




$\begin{array}{lllllll}\text { Pd36 } & 1.0000 & 0.00609 & 0.74968 & 0.00183 & \text { Biso } & 1.000 \mathrm{Pd} \\ \mathrm{C} 1 & 1.0000 & 0.80768 & 0.80786 & 0.45771 & \text { Biso } & 1.000 \mathrm{C} \\ \mathrm{C} 2 & 1.0000 & 0.65113 & 0.90592 & 0.46913 & \text { Biso } & 1.000 \mathrm{C} \\ \mathrm{C} 3 & 1.0000 & 0.01649 & 0.85550 & 0.46466 & \text { Biso } & 1.000 \mathrm{C} \\ \mathrm{H} 1 & 1.0000 & 0.91575 & 0.26833 & 0.37215 & \text { Biso } & 1.000 \mathrm{H} \\ \mathrm{H} 2 & 1.0000 & 0.78131 & 0.68034 & 0.45605 & \text { Biso } & 1.000 \mathrm{H} \\ \mathrm{H} 3 & 1.0000 & 0.51499 & 0.08413 & 0.38712 & \text { Biso } & 1.000 \mathrm{H} \\ \mathrm{H} 4 & 1.0000 & 0.10866 & 0.80162 & 0.43276 & \text { Biso } & 1.000 \mathrm{H} \\ \mathrm{H} 5 & 1.0000 & 0.50775 & 0.85678 & 0.47624 & \text { Biso } & 1.000 \mathrm{H} \\ \mathrm{H} 6 & 1.0000 & 0.67464 & 0.02624 & 0.48356 & \text { Biso } & 1.000 \mathrm{H} \\ \mathrm{H} 7 & 1.0000 & 0.03526 & 0.98388 & 0.46292 & \text { Biso } & 1.000 \mathrm{H} \\ \mathrm{H} 8 & 1.0000 & 0.06880 & 0.81338 & 0.50466 & \text { Biso } & 1.000 \mathrm{H}\end{array}$

\# Pd1.A2.P; $E=-118.82 ; Z P V E=2.84 \mathrm{eV}$; frequencies=22.93i, 3056.72, 3050.71, 3046.31, 3037.67, 2992.43, 2979.90, 2976.81, 2883.41, $1491.75,1489.91,1478.04,1471.99,1454.04,1403.58,1384.10,1338.29,1287.40,1188.86,1156.38,1042.52,920.78,897.62,857.70$, $737.04,398.24,369.70,324.32,320.09,280.06,229.28,64.35,54.61,46.57,27.16,21.21 \mathrm{~cm}^{\wedge}-1$

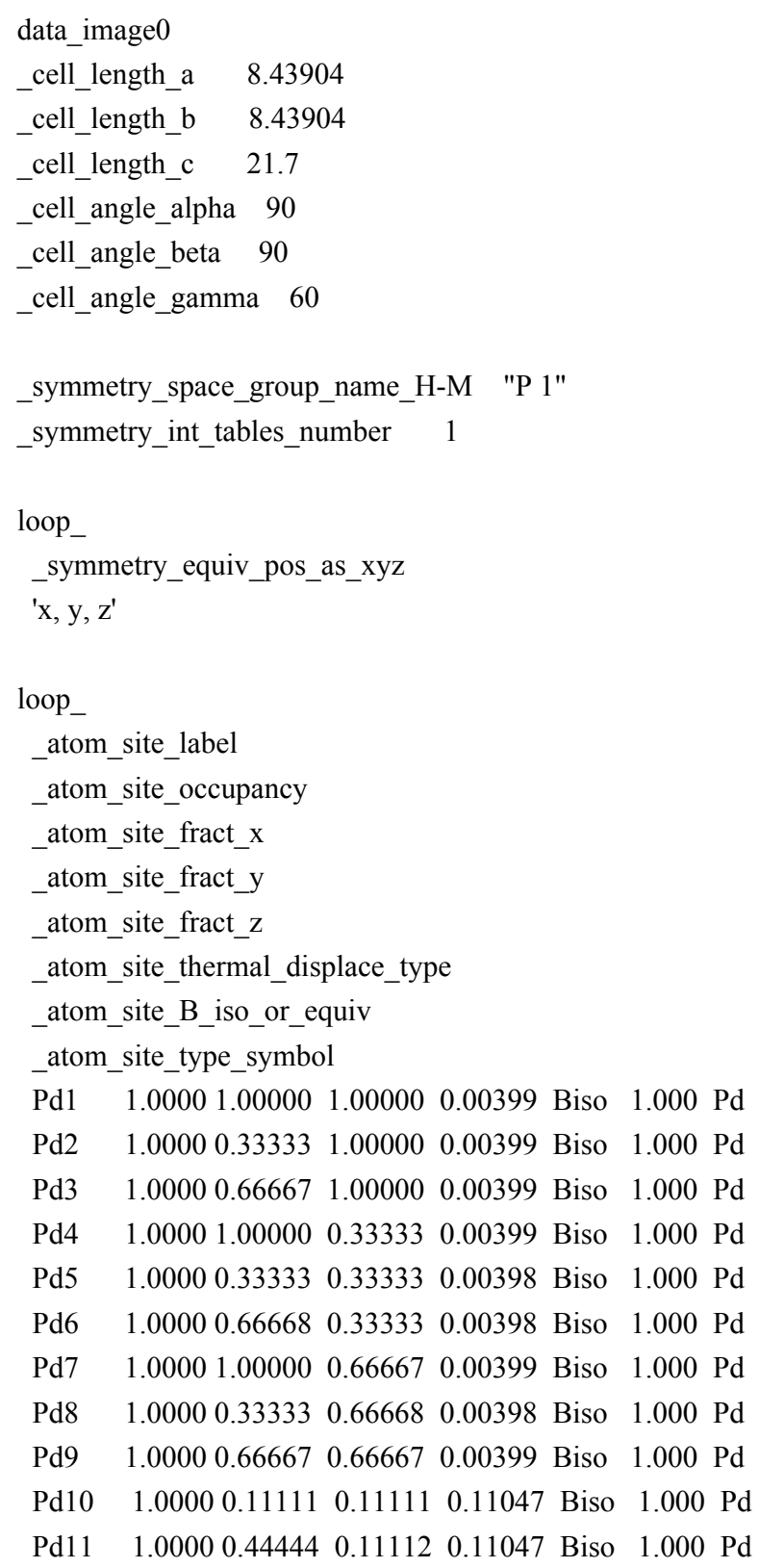




\begin{tabular}{|c|c|c|c|c|}
\hline Pd12 & 1.00000 .777770 .11112 & 0.11047 & Biso & 1.000 \\
\hline Pd13 & 1.00000 .111120 .44444 & 0.11047 & Biso & 1.00 \\
\hline Pd14 & 1.00000 .444440 .44444 & 0.11045 & Biso & 1.000 \\
\hline Pd15 & $\begin{array}{lll}1.0000 & 0.77777 & 0.44444\end{array}$ & 0.11047 & Biso & 1.000 \\
\hline Pd16 & $\begin{array}{lll}1.0000 & 0.11112 & 0.77777\end{array}$ & 0.11047 & Biso & 1.000 \\
\hline Pd17 & 1.00000 .444440 .77777 & 0.11047 & Biso & 1.000 \\
\hline Pd18 & $\begin{array}{lll}1.0000 & 0.77778 & 0.77778\end{array}$ & 0.11047 & Biso & 1.000 \\
\hline Pd19 & $\begin{array}{lll}1.0000 & 0.88914 & 0.22237\end{array}$ & 0.21456 & Biso & 1.000 \\
\hline $\operatorname{Pd} 20$ & $\begin{array}{lll}1.0000 & 0.22333 & 0.22232\end{array}$ & 0.21625 & Biso & 1.000 \\
\hline $\operatorname{Pd} 21$ & $1.0000 \quad 0.555340 .22209$ & 0.21653 & Biso & 1.000 \\
\hline $\operatorname{Pd} 22$ & $\begin{array}{lll}1.0000 & 0.88869 & 0.55692\end{array}$ & 0.21644 & Biso & 1.000 \\
\hline $\operatorname{Pd} 23$ & $\begin{array}{lll}1.0000 & 0.22237 & 0.55584\end{array}$ & 0.21573 & Biso & 1.000 \\
\hline $\operatorname{Pd} 24$ & $\begin{array}{lll}1.0000 & 0.55546 & 0.55672\end{array}$ & 0.21647 & Biso & 1.000 \\
\hline $\mathrm{Pd} 25$ & $\begin{array}{lll}1.0000 & 0.88885 & 0.88873\end{array}$ & 0.21658 & Biso & 1.000 \\
\hline $\operatorname{Pd} 26$ & $\begin{array}{lll}1.0000 & 0.22323 & 0.88867\end{array}$ & 0.21629 & Biso & 1.000 \\
\hline $\operatorname{Pd} 27$ & $\begin{array}{lll}1.0000 & 0.55605 & 0.88911\end{array}$ & 0.21538 & Biso & 1.000 \\
\hline $\operatorname{Pd} 28$ & $\begin{array}{lll}1.0000 & 0.99881 & 0.00455\end{array}$ & 0.32187 & Biso & 1.000 \\
\hline $\operatorname{Pd} 29$ & $\begin{array}{lll}1.0000 & 0.33545 & 0.99998\end{array}$ & 0.32173 & Biso & 1.000 \\
\hline $\operatorname{Pd} 30$ & 1.00000 .666780 .00006 & 0.32256 & Biso & 1.000 \\
\hline $\operatorname{Pd} 31$ & $\begin{array}{lll}1.0000 & 0.99850 & 0.33232\end{array}$ & 0.32134 & Biso & 1.000 \\
\hline $\operatorname{Pd} 32$ & $\begin{array}{lll}1.0000 & 0.33711 & 0.32788\end{array}$ & 0.32531 & Biso & 1.000 \\
\hline Pd33 & $\begin{array}{lll}1.0000 & 0.67128 & 0.33190\end{array}$ & 0.32178 & Biso & 1.000 \\
\hline $\operatorname{Pd} 34$ & 1.00000 .994140 .67049 & 0.32556 & Biso & 1.000 \\
\hline $\operatorname{Pd} 35$ & $\begin{array}{lll}1.0000 & 0.33729 & 0.66981\end{array}$ & 0.32497 & Biso & 1.000 \\
\hline $\operatorname{Pd} 36$ & $\begin{array}{lll}1.0000 & 0.66676 & 0.66832\end{array}$ & 0.32257 & Biso & 1.000 \\
\hline $\mathrm{O} 1$ & 1.00000 .222590 .55658 & 0.37708 & Biso & 1.000 \\
\hline $\mathrm{C} 1$ & 1.00000 .120160 .05272 & 0.48655 & Biso & 1.000 \\
\hline $\mathrm{C} 2$ & 1.00000 .280390 .86289 & 0.50210 & Biso & 1.000 \\
\hline $\mathrm{C} 3$ & 1.00000 .163180 .20668 & 0.49711 & Biso & 1.000 \\
\hline $\mathrm{H} 1$ & 1.00000 .999920 .07893 & 0.51376 & Biso & 1.000 \\
\hline $\mathrm{H} 2$ & $1.00000 .08106 \quad 0.05451$ & 0.43797 & Biso & 1.000 \\
\hline H3 & $\begin{array}{llll}1.0000 & 0.24671 & 0.75560\end{array}$ & 0.49303 & Biso & 1.000 \\
\hline H4 & 1.00000 .047440 .34029 & 0.48462 & Biso & 1.000 \\
\hline H5 & $1.0000 \quad 0.401350 .83225$ & 0.47459 & Biso & 1.000 \\
\hline H6 & $\begin{array}{lll}1.0000 & 0.31837 & 0.85469\end{array}$ & 0.55072 & Biso & 1.000 \\
\hline H7 & $\begin{array}{lll}1.0000 & 0.19600 & 0.21280\end{array}$ & 0.54554 & Biso & 1.000 \\
\hline H8 & $\begin{array}{lll}1.0000 & 0.28100 & 0.18550\end{array}$ & 0.46887 & Biso & 1.000 \\
\hline
\end{tabular}

\# Pd1.A2.T3; $E=-117.32 ; \mathrm{ZPVE}=2.67 \mathrm{eV}$; frequencies= 1731.54i, 3061.59, 3043.50, 3018.99, 3005.66, 2964.40, 2925.08, 2923.28, 1486.62, 1479.54, 1459.60, 1445.08, 1386.89, 1331.35, 1293.93, 1253.74, 1221.06, 1168.30, 1096.94, 1016.69, 985.66, 911.83, 874.93, $754.39,559.57,526.71,401.97,335.40,299.70,279.43,194.95,152.81,102.25,79.41,41.25,35.86 \mathrm{~cm}^{\wedge}-1$

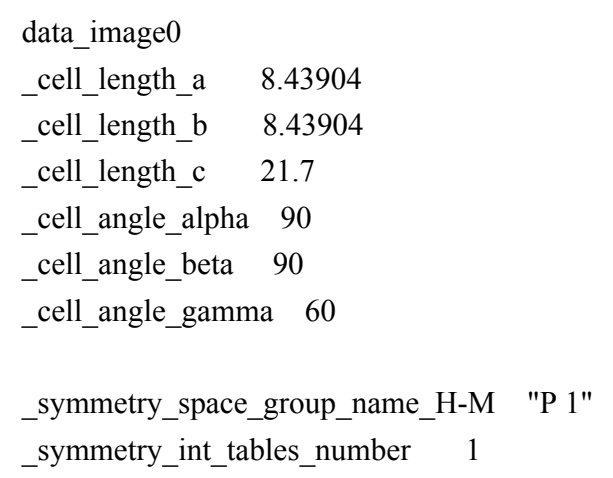




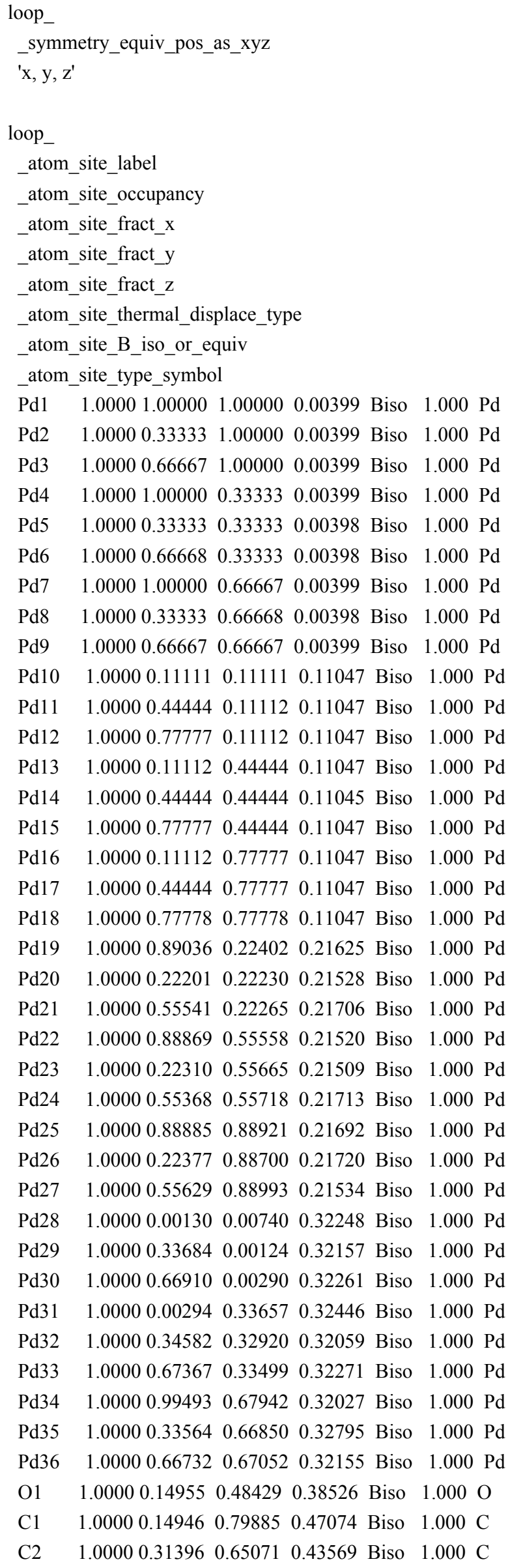


C3 $\quad 1.00000 .207610 .86914 \quad 0.52668$ Biso $1.000 \mathrm{C}$

H1 $1.00000 .06038 \quad 0.74598 \quad 0.48530$ Biso $1.000 \mathrm{H}$

$\mathrm{H} 2 \quad 1.00000 .067170 .914530 .43977$ Biso $1.000 \mathrm{H}$

H3 $1.0000 \quad 0.234260 .567840 .40227$ Biso $1.000 \mathrm{H}$

H4 $1.00000 .08750 \quad 0.973590 .55129$ Biso $1.000 \mathrm{H}$

H5 $1.00000 .41190 \quad 0.697170 .42533$ Biso $1.000 \mathrm{H}$

H6 1.00000 .392150 .522770 .46154 Biso $1.000 \mathrm{H}$

H7 $\quad \begin{array}{lllllll}1.0000 & 0.28909 & 0.75820 & 0.55896 & \text { Biso } 1.000 \mathrm{H}\end{array}$

H8 $\quad \begin{array}{llllll}1.0000 & 0.28972 & 0.93008 & 0.51098 & \text { Biso } 1.000 \mathrm{H}\end{array}$

\# Pd1.A2.1p; $\mathrm{E}=-118.63 ; \mathrm{ZPVE}=2.82 \mathrm{eV}$; frequencies=9.04i, 3665.21, 3075.10, 3067.51, 3064.04, 3005.47, 2989.94, 2982.40, 2956.85, $1487.04,1477.14,1459.69,1422.40,1379.18,1294.89,1287.55,1204.02,1109.35,1021.49,974.99,941.77,884.21,776.44,649.87,626.46$, $605.68,526.61,316.18,245.82,236.07,191.05,171.08,148.45,116.28,49.58,43.95 \mathrm{~cm}^{\wedge}-1$

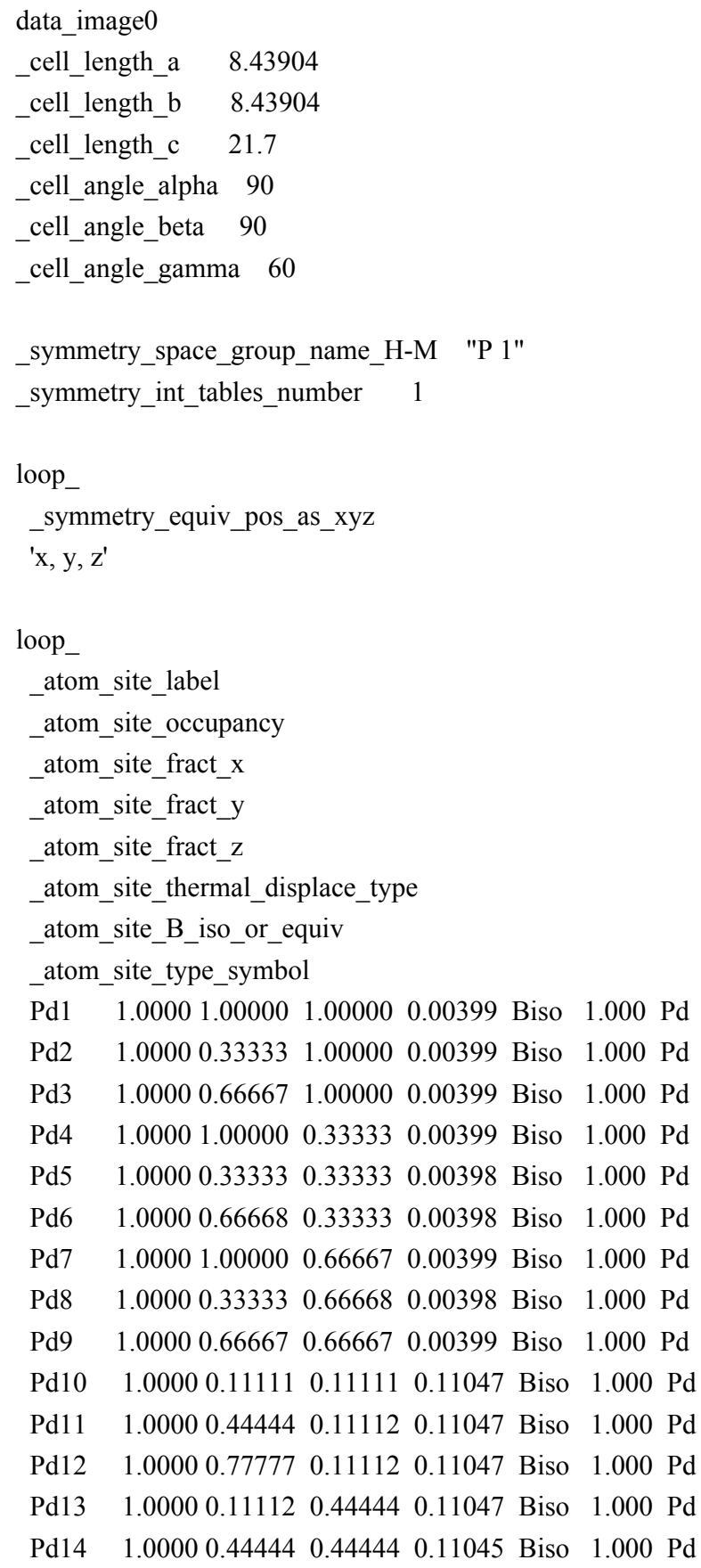




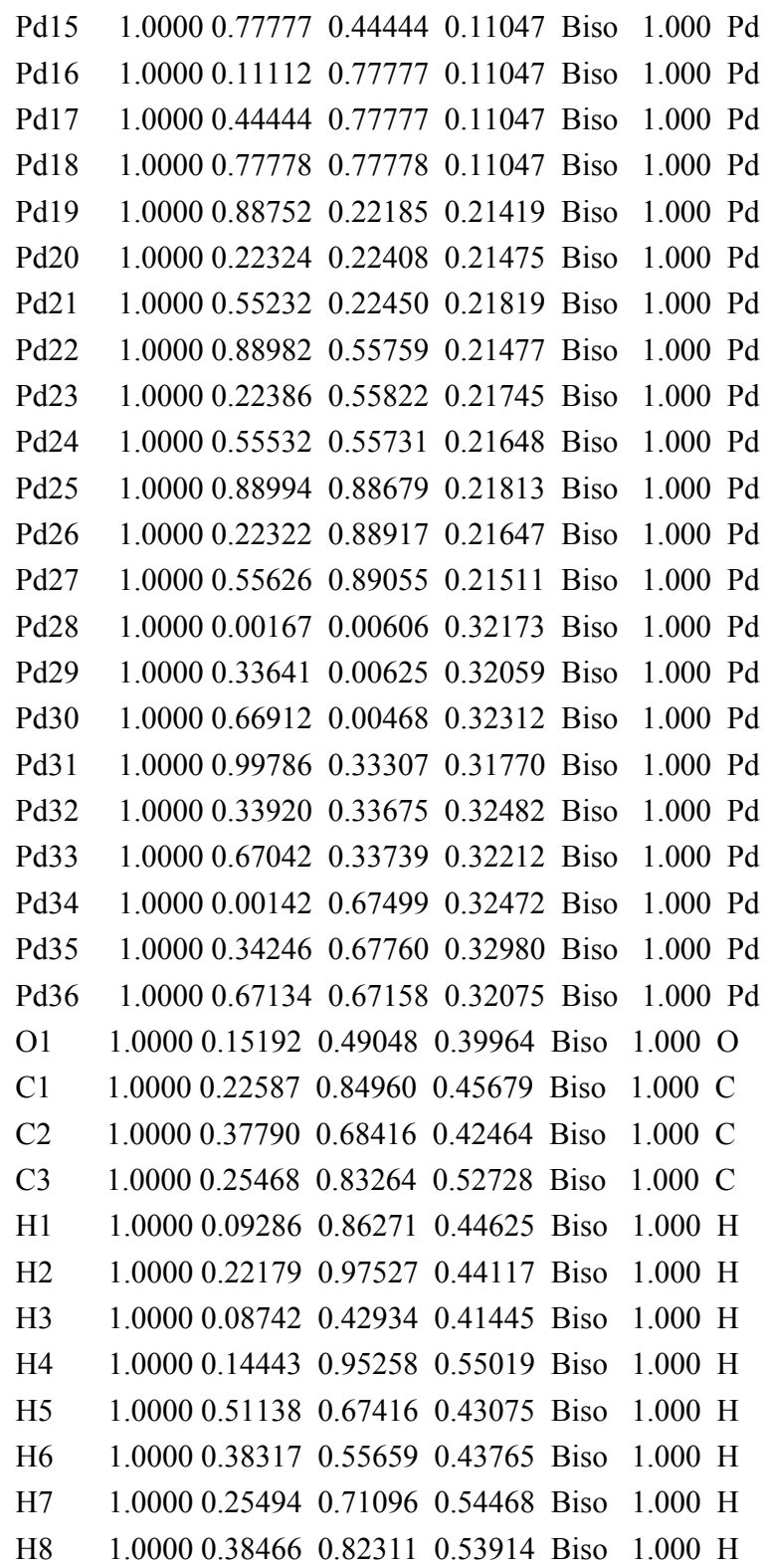

\# Pd1.A2.T4; $E=-117.97 ; \mathrm{ZPVE}=2.66 \mathrm{eV}$; frequencies= 1234.67i, 3703.43, 3075.69, 3070.30, 3038.00, 2995.35, 2983.95, 2975.49, 1482.12 , 1476.81, 1436.71, 1424.02, 1388.42, 1357.38, 1224.18, 1189.94, 1145.04, 1055.14, 970.22, 950.44, 916.56, 878.03, 760.27, 654.00, $581.39,431.60,395.24,321.88,316.78,204.24,166.80,143.71,82.68,76.41,56.35,27.28 \mathrm{~cm}^{\wedge}-1$

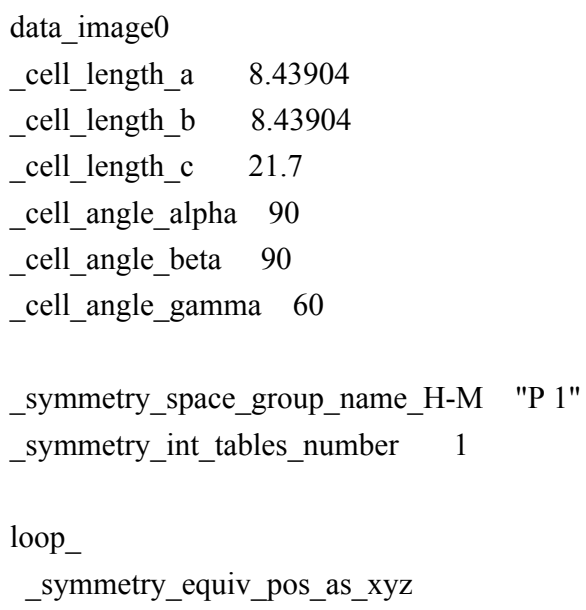




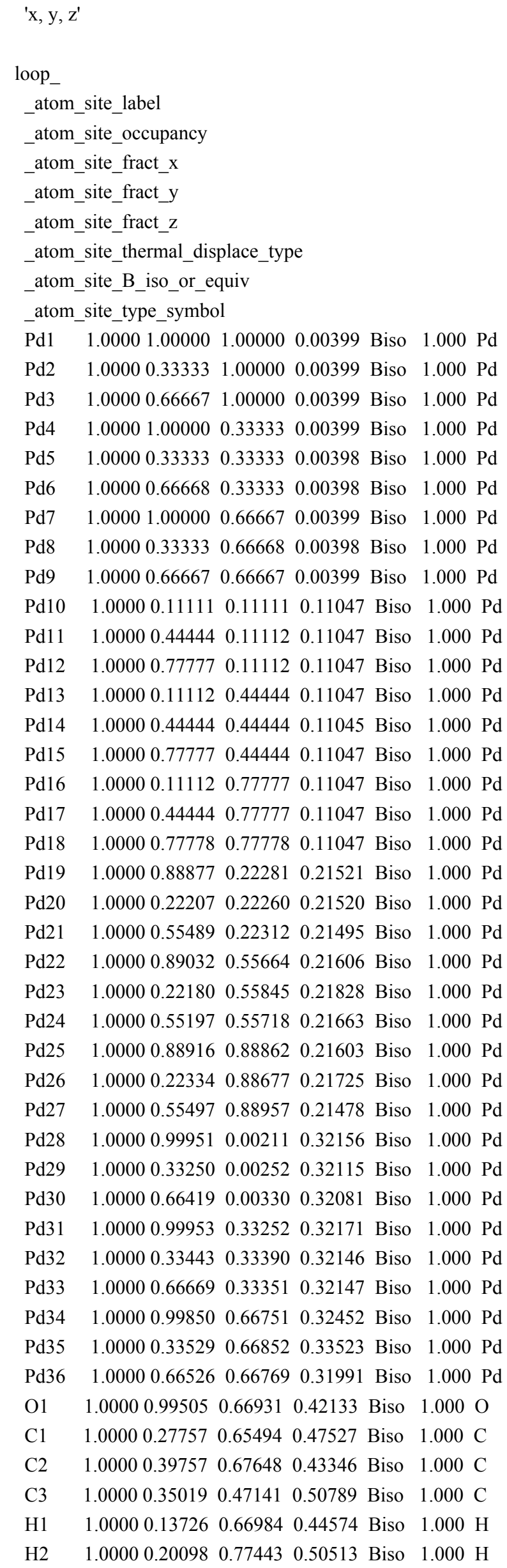




$\begin{array}{llllllll}\text { H3 } & 1.0000 & 0.88894 & 0.78261 & 0.43483 & \text { Biso } & 1.000 \mathrm{H} \\ \text { H4 } & 1.0000 & 0.24058 & 0.46364 & 0.53192 & \text { Biso } & 1.000 \mathrm{H} \\ \text { H5 } & 1.0000 & 0.38729 & 0.81052 & 0.43205 & \text { Biso } & 1.000 \mathrm{H} \\ \text { H6 } & 1.0000 & 0.53743 & 0.56169 & 0.42939 & \text { Biso } & 1.000 \mathrm{H} \\ \text { H7 } & 1.0000 & 0.41092 & 0.35867 & 0.47472 & \text { Biso } & 1.000 \mathrm{H} \\ \text { H8 } & 1.0000 & 0.45553 & 0.44975 & 0.54106 & \text { Biso } & 1.000 \mathrm{H}\end{array}$

\# Pd1.A2.y; $\mathrm{E}=-119.34 ; \mathrm{ZPVE}=2.77 \mathrm{eV}$; frequencies=13.55i, 3826.80, 3601.64, 3151.76, 3110.71, 3076.05, 3061.08, 3019.16, 2964.12, $1602.16,1517.37,1468.57,1450.86,1403.49,1380.28,1254.87,1172.51,1024.84,970.98,916.56,910.35,883.39,700.17,408.34,343.50$, $277.36,242.29,194.91,152.65,144.31,104.70,85.84,73.65,63.88,46.14,11.52 \mathrm{~cm}^{\wedge}-1$

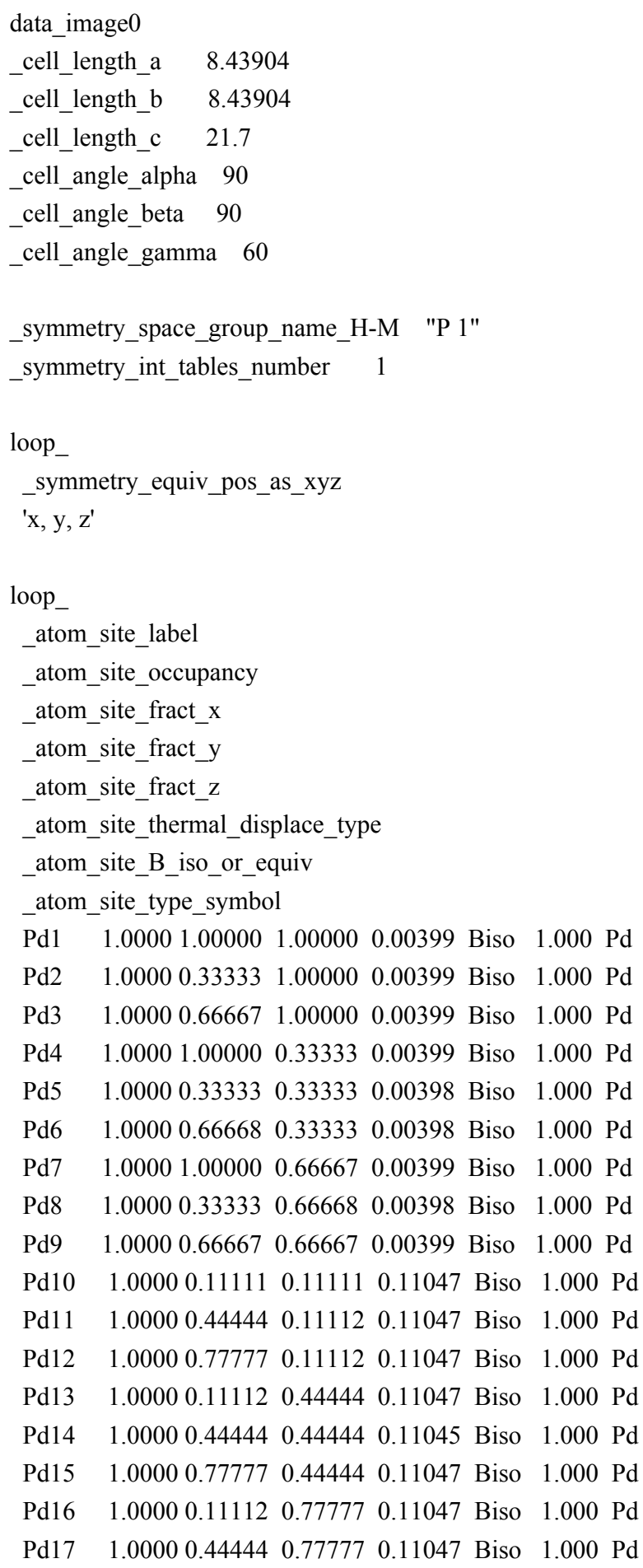




$\begin{array}{lllllll}\mathrm{Pd} 18 & 1.0000 & 0.77778 & 0.77778 & 0.11047 & \text { Biso } & 1.000 \mathrm{Pd} \\ \mathrm{Pd} 19 & 1.0000 & 0.88820 & 0.22218 & 0.21528 & \text { Biso } & 1.000 \mathrm{Pd} \\ \mathrm{Pd} 20 & 1.0000 & 0.22173 & 0.22203 & 0.21516 & \text { Biso } & 1.000 \mathrm{Pd} \\ \mathrm{Pd} 21 & 1.0000 & 0.55516 & 0.22199 & 0.21504 & \text { Biso } & 1.000 \mathrm{Pd} \\ \mathrm{Pd} 22 & 1.0000 & 0.88866 & 0.55537 & 0.21543 & \text { Biso } & 1.000 \mathrm{Pd} \\ \mathrm{Pd} 23 & 1.0000 & 0.22384 & 0.55759 & 0.21819 & \text { Biso } & 1.000 \mathrm{Pd} \\ \mathrm{Pd} 24 & 1.0000 & 0.55198 & 0.55672 & 0.21765 & \text { Biso } & 1.000 \mathrm{Pd} \\ \mathrm{Pd} 25 & 1.0000 & 0.88848 & 0.88849 & 0.21512 & \text { Biso } & 1.000 \mathrm{Pd} \\ \mathrm{Pd} 26 & 1.0000 & 0.22293 & 0.88595 & 0.21715 & \text { Biso } & 1.000 \mathrm{Pd} \\ \mathrm{Pd} 27 & 1.0000 & 0.55517 & 0.88834 & 0.21492 & \text { Biso } & 1.000 \mathrm{Pd} \\ \mathrm{Pd} 28 & 1.0000 & 0.99749 & 0.00052 & 0.32125 & \text { Biso } & 1.000 \mathrm{Pd} \\ \mathrm{Pd} 29 & 1.0000 & 0.33158 & 0.00117 & 0.32077 & \text { Biso } & 1.000 \mathrm{Pd} \\ \mathrm{Pd} 30 & 1.0000 & 0.66533 & 0.99920 & 0.32067 & \text { Biso } & 1.000 \mathrm{Pd} \\ \mathrm{Pd} 31 & 1.0000 & 0.99882 & 0.33232 & 0.32224 & \text { Biso } & 1.000 \mathrm{Pd} \\ \mathrm{Pd} 32 & 1.0000 & 0.33223 & 0.33048 & 0.32089 & \text { Biso } & 1.000 \mathrm{Pd} \\ \mathrm{Pd} 33 & 1.0000 & 0.66720 & 0.33069 & 0.32165 & \text { Biso } & 1.000 \mathrm{Pd} \\ \mathrm{Pd} 34 & 1.0000 & 0.99696 & 0.66590 & 0.32153 & \text { Biso } & 1.000 \mathrm{Pd} \\ \mathrm{Pd} 35 & 1.0000 & 0.33221 & 0.66473 & 0.33541 & \text { Biso } & 1.000 \mathrm{Pd} \\ \mathrm{Pd} 36 & 1.0000 & 0.66788 & 0.66510 & 0.32118 & \text { Biso } & 1.000 \mathrm{Pd} \\ \mathrm{O} 1 & 1.0000 & 0.78587 & 0.97183 & 0.47821 & \text { Biso } & 1.000 \mathrm{O} \\ \mathrm{C} 1 & 1.0000 & 0.27327 & 0.65902 & 0.43833 & \text { Biso } & 1.000 \mathrm{C} \\ \mathrm{C} 2 & 1.0000 & 0.39802 & 0.71991 & 0.43062 & \text { Biso } & 1.000 \mathrm{C} \\ \mathrm{C} 3 & 1.0000 & 0.32502 & 0.47399 & 0.46408 & \text { Biso } & 1.000 \mathrm{C} \\ \mathrm{H} 1 & 1.0000 & 0.75454 & 0.98485 & 0.43459 & \text { Biso } & 1.000 \mathrm{H} \\ \mathrm{H} 2 & 1.0000 & 0.12907 & 0.76158 & 0.44185 & \text { Biso } & 1.000 \mathrm{H} \\ \mathrm{H} 3 & 1.0000 & 0.24391 & 0.41872 & 0.44389 & \text { Biso } & 1.000 \mathrm{H} \\ \mathrm{H} 4 & 1.0000 & 0.71050 & 0.92814 & 0.49658 & \text { Biso } & 1.000 \mathrm{H} \\ \mathrm{H} 5 & 1.0000 & 0.35127 & 0.86547 & 0.43156 & \text { Biso } & 1.000 \mathrm{H} \\ \mathrm{H} 6 & 1.0000 & 0.54224 & 0.62884 & 0.44028 & \text { Biso } & 1.000 \mathrm{H} \\ \mathrm{H} 7 & 1.0000 & 0.47021 & 0.37486 & 0.45840 & \text { Biso } & 1.000 \mathrm{H} \\ & 1.0000 & 0.29429 & 0.49018 & 0.51296 & \text { Biso } & 1.000 \mathrm{H}\end{array}$

\# Pd1.B2.P; $E=-119.02 ; \mathrm{ZPVE}=2.84 \mathrm{eV}$; frequencies= 7.97i, 19.27i, 3058.02, 3054.55, 3042.56, 3025.29, 3010.55, 2987.60, 2972.52, $2966.45,1496.52,1490.48,1480.38,1471.66,1469.28,1402.50,1381.52,1350.00,1302.31,1193.03,1153.76,1044.20,918.57,900.91$, $858.38,747.40,399.24,367.27,327.54,323.59,270.37,220.44,45.63,32.49,24.58,12.56 \mathrm{~cm}^{\wedge}-1$

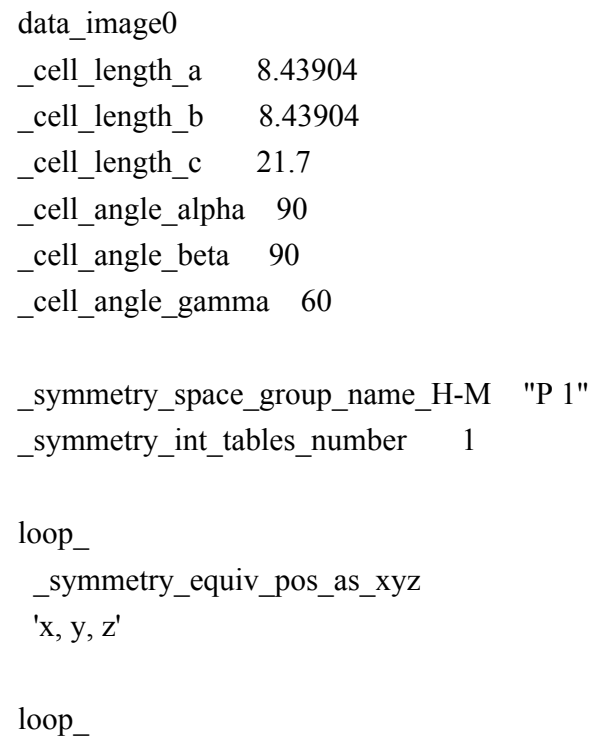




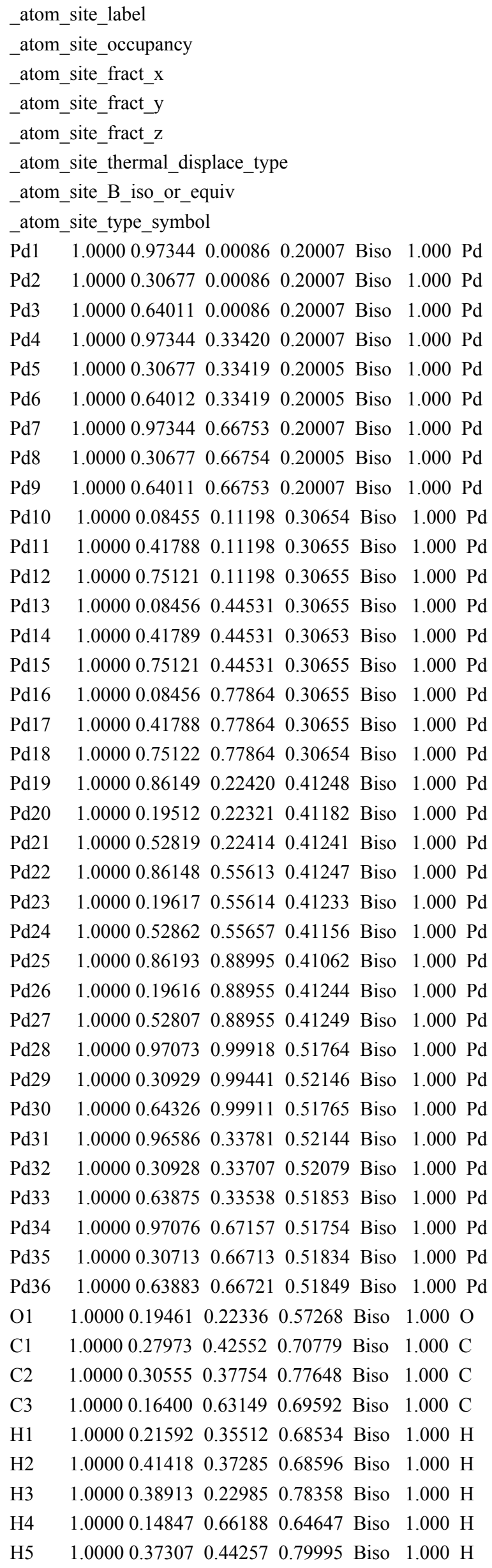


H6 $\quad 1.00000 .173730 .42380 \quad 0.79918$ Biso $1.000 \mathrm{H}$

H7 $1.00000 .02657 \quad 0.68757 \quad 0.71547$ Biso $1.000 \mathrm{H}$

H8 $\quad 1.00000 .22660 \quad 0.70555 \quad 0.71580$ Biso $1.000 \mathrm{H}$

\# Pd1.B2.T3; $\mathrm{E}=-117.36 ; \mathrm{ZPVE}=2.66 \mathrm{eV} ;$ frequencies $=1723.67 \mathrm{i}, 3061.38,3057.67,3022.78,3020.19,2957.49,2952.72,2908.74$, $1477.57,1462.26,1461.21,1450.76,1387.44,1369.09,1285.25,1234.02,1188.92,1180.57,1097.25,1075.65,932.52,912.87,895.31$, $826.99,473.79,417.49,364.54,304.49,238.50,203.11,178.40,175.76,129.47,65.87,57.70,53.85 \mathrm{~cm}^{\wedge}-1$

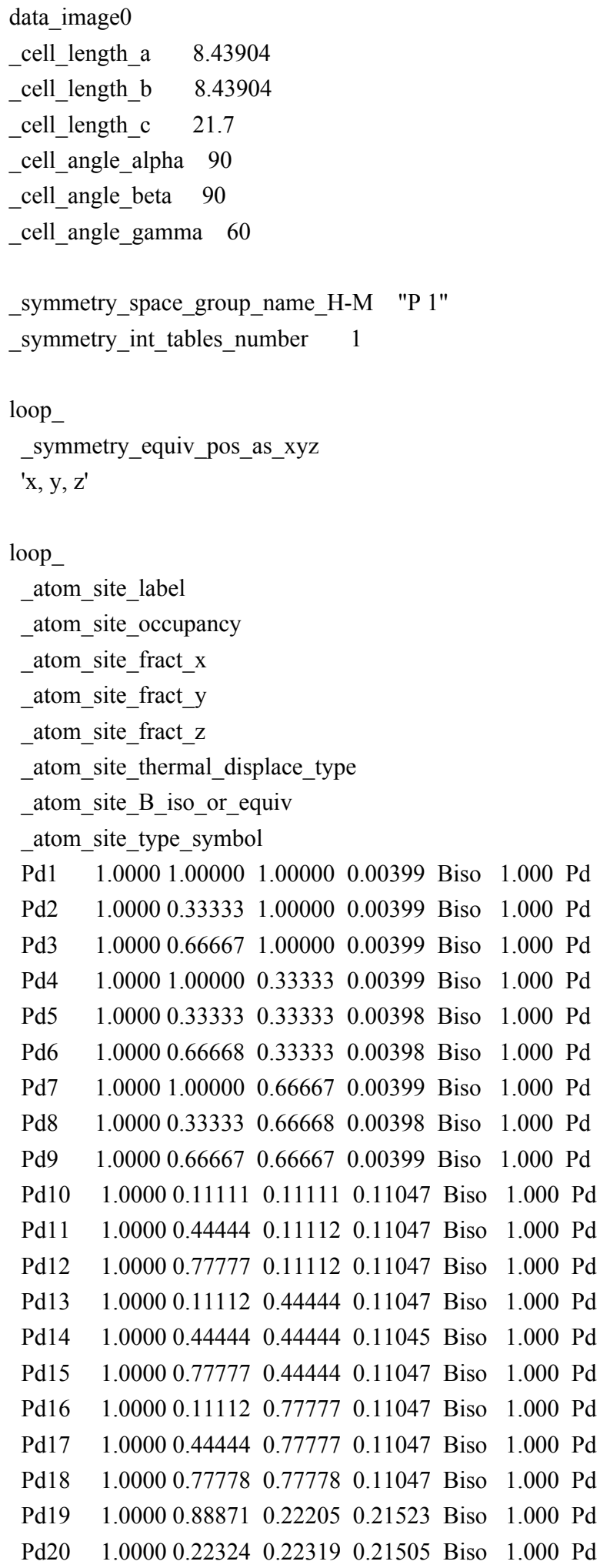




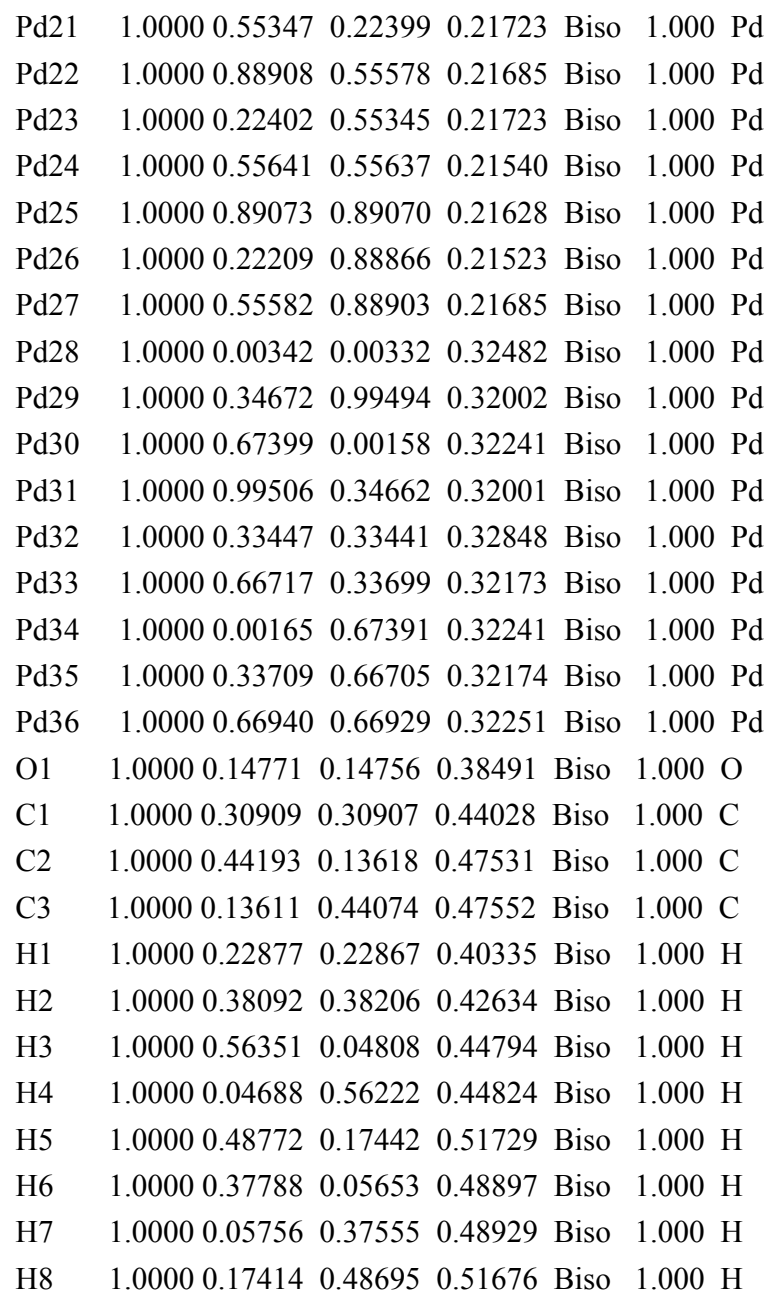

\# Pd1.B2.2p; $E=-118.66 ; Z P V E=2.80 \mathrm{eV}$; frequencies=3651.26, 3082.99, 3079.53, 3002.00, 2990.41, 2959.79, 2934.89, 2928.10, 1474.53, $1454.00,1453.62,1439.44,1377.27,1362.84,1321.01,1175.41,1086.72,1083.96,913.94,908.15,882.32,847.86,621.79,593.89,418.23$, $387.40,316.68,236.31,212.73,202.31,197.50,176.11,173.19,80.37,77.53,56.17 \mathrm{~cm}^{\wedge}-1$

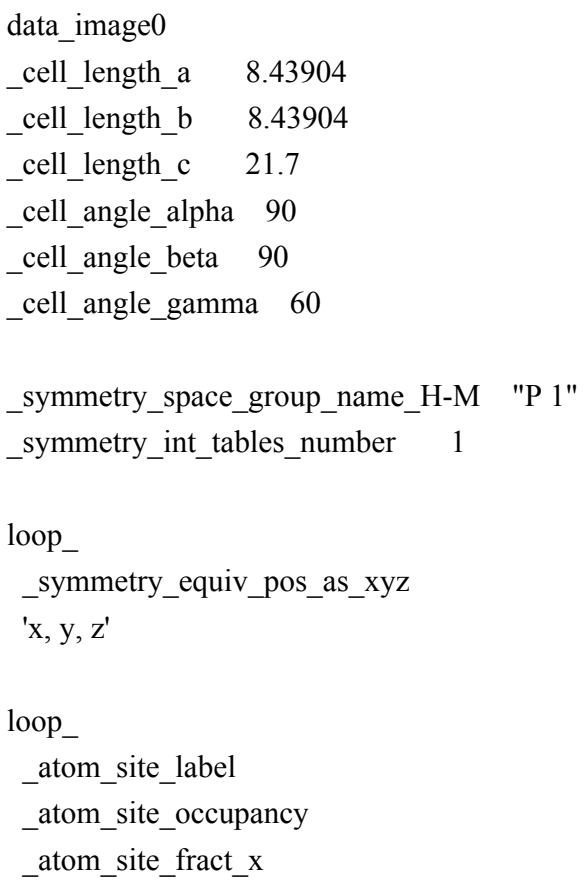




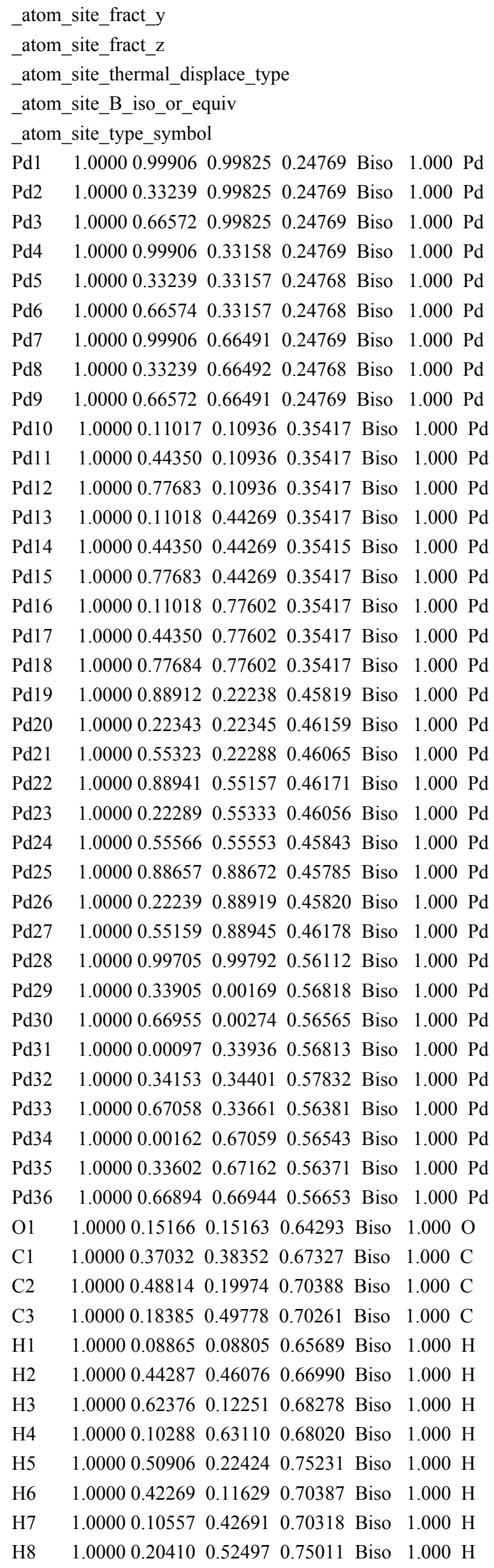


\# Pd1.B2.T4; E= -117.98; ZPVE= $2.65 \mathrm{eV}$; frequencies=1289.92i, 3703.14, 3106.93, 3056.14, 3003.53, 2988.88, 2948.01, 2941.19, 1480.26, 1453.54, 1445.61, 1410.14, 1378.44, 1357.69, 1309.98, 1190.30, 1150.62, 1013.36, 983.02, 940.29, 888.08, 815.29, 786.23, 571.89, $563.03,428.33,402.53,337.44,314.84,185.51,169.06,143.93,95.82,74.23,54.93,52.34 \mathrm{~cm}^{\wedge}-1$

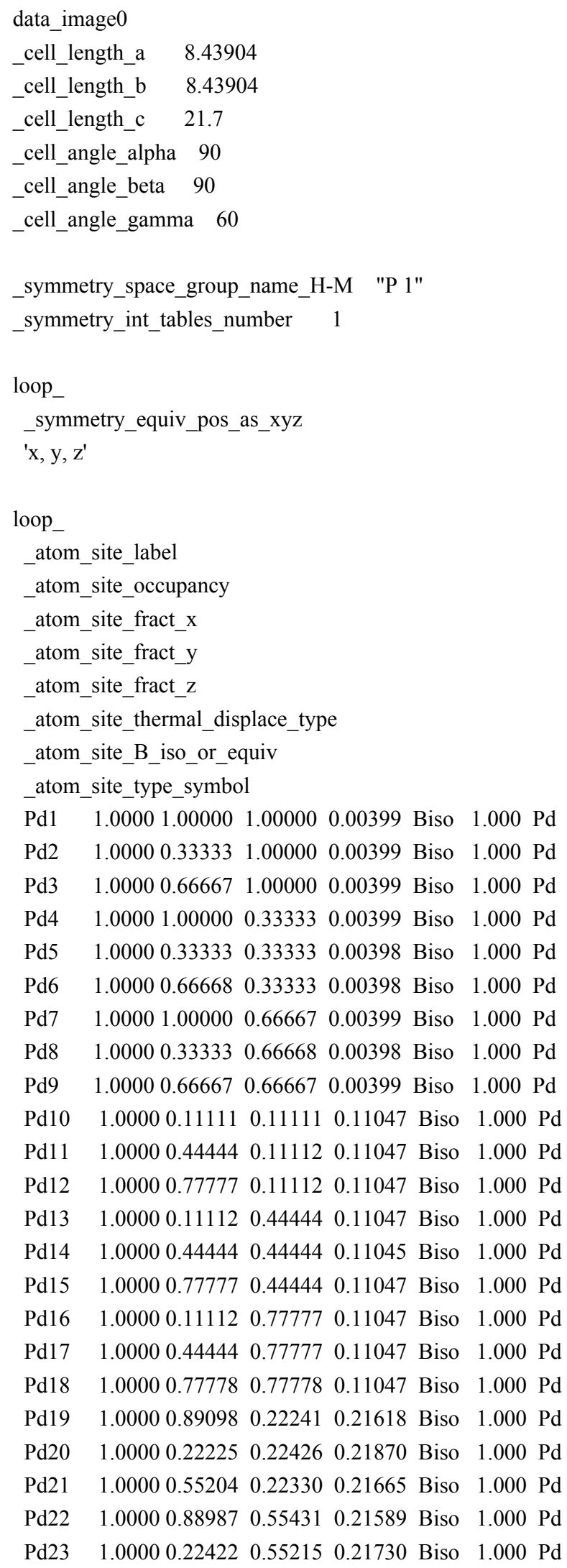




$\begin{array}{llllllll}\mathrm{Pd} 24 & 1.0000 & 0.55547 & 0.55529 & 0.21471 & \text { Biso } & 1.000 \mathrm{Pd} \\ \mathrm{Pd} 25 & 1.0000 & 0.88948 & 0.88857 & 0.21495 & \text { Biso } & 1.000 \mathrm{Pd} \\ \mathrm{Pd} 26 & 1.0000 & 0.22265 & 0.88833 & 0.21520 & \text { Biso } & 1.000 \mathrm{Pd} \\ \mathrm{Pd} 27 & 1.0000 & 0.55521 & 0.88888 & 0.21478 & \text { Biso } & 1.000 \mathrm{Pd} \\ \mathrm{Pd} 28 & 1.0000 & 0.00089 & 0.99717 & 0.32170 & \text { Biso } & 1.000 \mathrm{Pd} \\ \mathrm{Pd} 29 & 1.0000 & 0.33534 & 0.99851 & 0.32163 & \text { Biso } & 1.000 \mathrm{Pd} \\ \mathrm{Pd} 30 & 1.0000 & 0.66809 & 0.99818 & 0.32058 & \text { Biso } & 1.000 \mathrm{Pd} \\ \mathrm{Pd} 31 & 1.0000 & 0.00000 & 0.33153 & 0.32491 & \text { Biso } & 1.000 \mathrm{Pd} \\ \mathrm{Pd} 32 & 1.0000 & 0.33756 & 0.33129 & 0.33665 & \text { Biso } & 1.000 \mathrm{Pd} \\ \mathrm{Pd} 33 & 1.0000 & 0.66615 & 0.33285 & 0.32006 & \text { Biso } & 1.000 \mathrm{Pd} \\ \mathrm{Pd} 34 & 1.0000 & 0.00117 & 0.66666 & 0.32117 & \text { Biso } & 1.000 \mathrm{Pd} \\ \mathrm{Pd} 35 & 1.0000 & 0.33410 & 0.66676 & 0.32125 & \text { Biso } & 1.000 \mathrm{Pd} \\ \mathrm{Pd} 36 & 1.0000 & 0.66572 & 0.66770 & 0.32050 & \text { Biso } & 1.000 \mathrm{Pd} \\ \mathrm{O} 1 & 1.0000 & 0.98963 & 0.34853 & 0.42205 & \text { Biso } & 1.000 \mathrm{O} \\ \mathrm{C} 1 & 1.0000 & 0.39214 & 0.33962 & 0.43972 & \text { Biso } & 1.000 \mathrm{C} \\ \mathrm{C} 2 & 1.0000 & 0.52332 & 0.14856 & 0.46182 & \text { Biso } & 1.000 \mathrm{C} \\ \mathrm{C} 3 & 1.0000 & 0.21770 & 0.43883 & 0.46919 & \text { Biso } & 1.000 \mathrm{C} \\ \mathrm{H} 1 & 1.0000 & 0.86761 & 0.44491 & 0.43344 & \text { Biso } & 1.000 \mathrm{H} \\ \mathrm{H} 2 & 1.0000 & 0.45546 & 0.41813 & 0.42340 & \text { Biso } & 1.000 \mathrm{H} \\ \mathrm{H} 3 & 1.0000 & 0.64081 & 0.07415 & 0.43106 & \text { Biso } & 1.000 \mathrm{H} \\ \mathrm{H} 4 & 1.0000 & 0.15196 & 0.58741 & 0.46719 & \text { Biso } & 1.000 \mathrm{H} \\ \mathrm{H} 5 & 1.0000 & 0.57813 & 0.15737 & 0.50690 & \text { Biso } & 1.000 \mathrm{H} \\ \mathrm{H} 6 & 1.0000 & 0.45502 & 0.06852 & 0.46791 & \text { Biso } & 1.000 \mathrm{H} \\ \mathrm{H} 7 & 1.0000 & 0.10201 & 0.39823 & 0.44372 & \text { Biso } & 1.000 \mathrm{H} \\ \mathrm{H} 8 & 1.0000 & 0.21089 & 0.38647 & 0.51399 & \text { Biso } & 1.000 \mathrm{H}\end{array}$

\# Pd1.B2.y; $E=-119.35 ; Z P V E=2.77 \mathrm{eV}$; frequencies= 3829.49, 3620.05, 3166.80, 3094.42, 3076.59, 3066.14, 3010.50, 2958.89, 1605.89, $1516.96,1466.77,1451.87,1401.80,1380.62,1253.17,1172.84,1024.43,973.89,929.12,911.45,881.95,697.88,411.21,345.54,244.41$, $238.79,193.43,166.47,146.88,111.32,87.45,79.93,70.02,53.17,42.83,8.62 \mathrm{~cm}^{\wedge}-1$

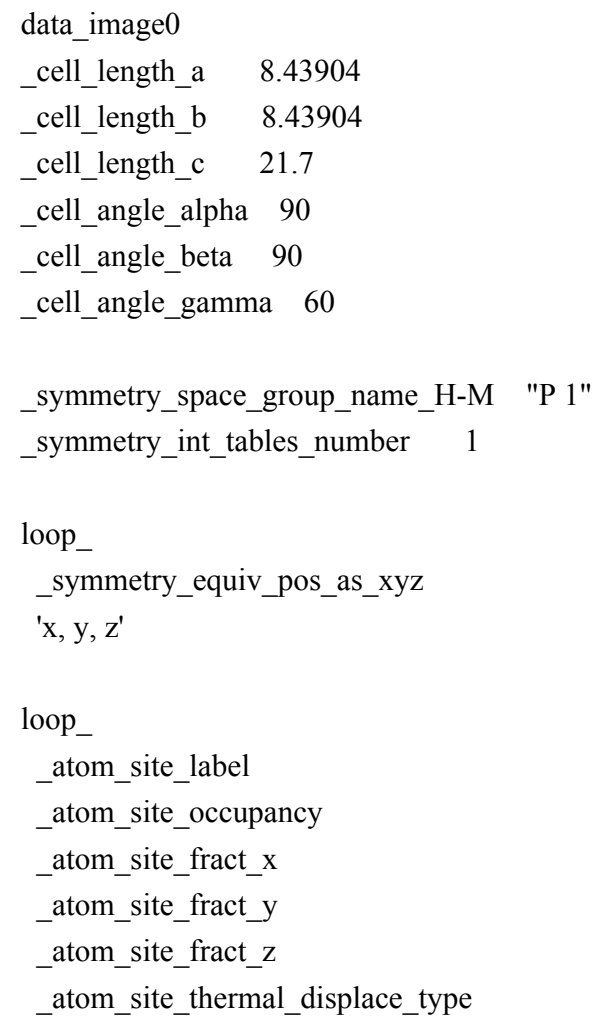




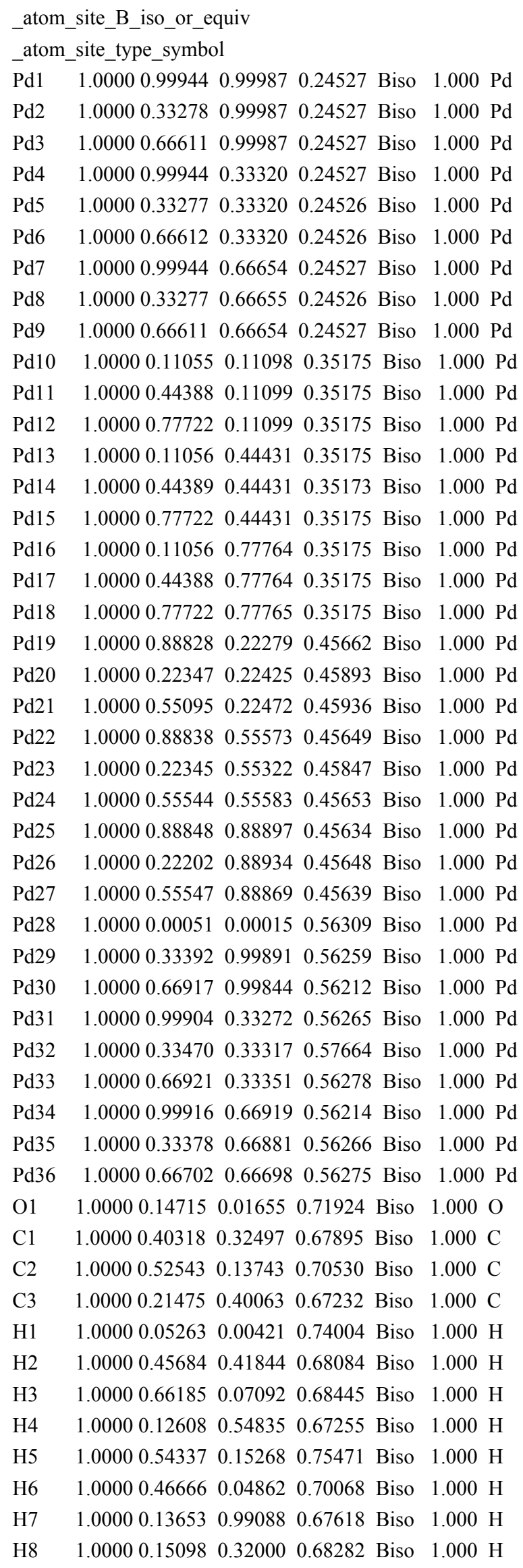


\# Pd2.A2.P; $\mathrm{E}=-118.45 ; \mathrm{ZPVE}=2.83 \mathrm{eV}$; frequencies= 3060.06, 3054.45, 3048.72, 3043.43, 2996.67, 2981.31, 2978.25, 2744.13, 1490.53, $1488.57,1478.23,1470.91,1423.68,1402.91,1385.33,1331.38,1258.14,1184.47,1158.61,1041.74,923.15,892.95,859.79,730.75$, $404.74,371.46,344.04,340.39,279.33,230.23,95.33,70.99,56.50,38.37,20.82,8.93 \mathrm{~cm}^{\wedge}-1$

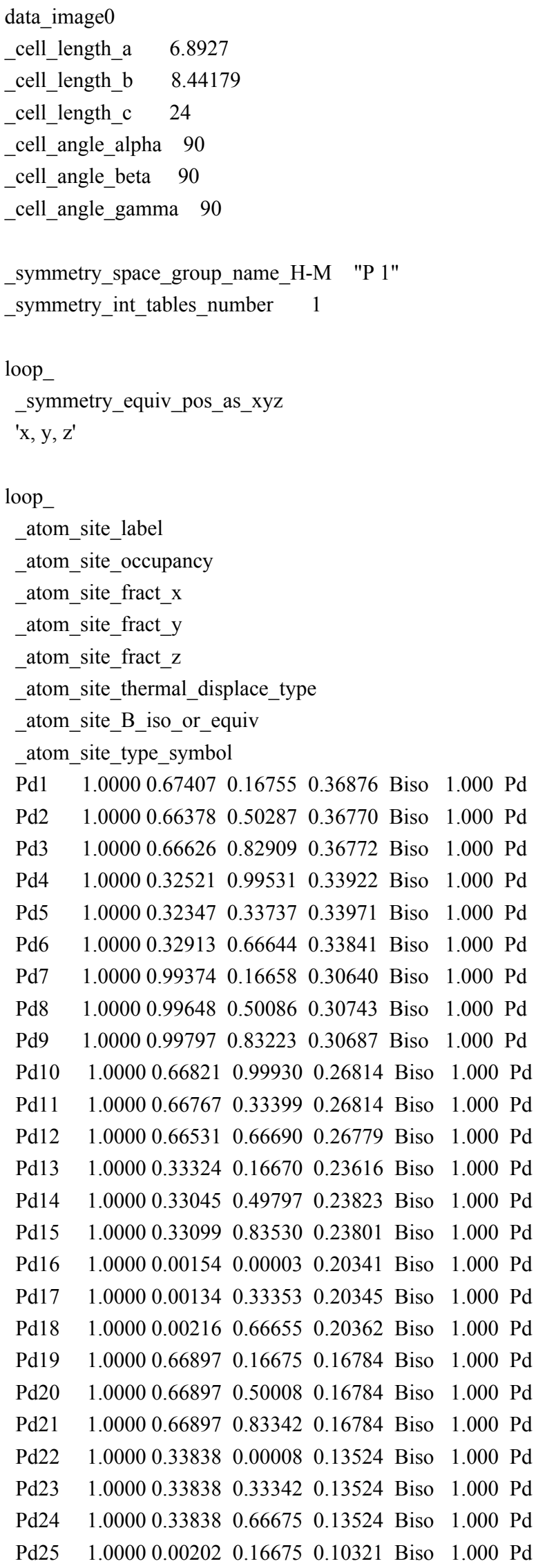




$\begin{array}{lllllll}\mathrm{Pd} 26 & 1.0000 & 0.00202 & 0.50008 & 0.10321 & \text { Biso } & 1.000 \mathrm{Pd} \\ \mathrm{Pd} 27 & 1.0000 & 0.00202 & 0.83342 & 0.10321 & \text { Biso } & 1.000 \mathrm{Pd} \\ \mathrm{Pd} 28 & 1.0000 & 0.66998 & 0.00008 & 0.06364 & \text { Biso } & 1.000 \mathrm{Pd} \\ \mathrm{Pd} 29 & 1.0000 & 0.66998 & 0.33342 & 0.06364 & \text { Biso } & 1.000 \mathrm{Pd} \\ \mathrm{Pd} 30 & 1.0000 & 0.66998 & 0.66675 & 0.06364 & \text { Biso } & 1.000 \mathrm{Pd} \\ \mathrm{Pd} 31 & 1.0000 & 0.33931 & 0.16675 & 0.03394 & \text { Biso } & 1.000 \mathrm{Pd} \\ \mathrm{Pd} 32 & 1.0000 & 0.33931 & 0.50008 & 0.03394 & \text { Biso } & 1.000 \mathrm{Pd} \\ \mathrm{Pd} 33 & 1.0000 & 0.33931 & 0.83342 & 0.03394 & \text { Biso } & 1.000 \mathrm{Pd} \\ \mathrm{Pd} 34 & 1.0000 & 0.00766 & 0.00008 & 0.00384 & \text { Biso } & 1.000 \mathrm{Pd} \\ \mathrm{Pd} 35 & 1.0000 & 0.00766 & 0.33342 & 0.00384 & \text { Biso } & 1.000 \mathrm{Pd} \\ \mathrm{Pd} 36 & 1.0000 & 0.00766 & 0.66675 & 0.00384 & \text { Biso } & 1.000 \mathrm{Pd} \\ \mathrm{O} 1 & 1.0000 & 0.39464 & 0.16594 & 0.39449 & \text { Biso } & 1.000 \mathrm{O} \\ \mathrm{C} 1 & 1.0000 & 0.84069 & 0.86403 & 0.49551 & \text { Biso } & 1.000 \mathrm{C} \\ \mathrm{C} 2 & 1.0000 & 0.76555 & 0.02297 & 0.51715 & \text { Biso } & 1.000 \mathrm{C} \\ \mathrm{C} 3 & 1.0000 & 0.06198 & 0.85054 & 0.49573 & \text { Biso } & 1.000 \mathrm{C} \\ \mathrm{H} 1 & 1.0000 & 0.78840 & 0.84688 & 0.45202 & \text { Biso } & 1.000 \mathrm{H} \\ \mathrm{H} 2 & 1.0000 & 0.77644 & 0.76672 & 0.51949 & \text { Biso } & 1.000 \mathrm{H} \\ \mathrm{H} 3 & 1.0000 & 0.60734 & 0.03011 & 0.51476 & \text { Biso } & 1.000 \mathrm{H} \\ \mathrm{H} 4 & 1.0000 & 0.11010 & 0.73711 & 0.47850 & \text { Biso } & 1.000 \mathrm{H} \\ \mathrm{H} 5 & 1.0000 & 0.80809 & 0.04089 & 0.56064 & \text { Biso } & 1.000 \mathrm{H} \\ \mathrm{H} 6 & 1.0000 & 0.82555 & 0.12132 & 0.49287 & \text { Biso } & 1.000 \mathrm{H} \\ \mathrm{H} 7 & 1.0000 & 0.12817 & 0.94522 & 0.47101 & \text { Biso } & 1.000 \mathrm{H} \\ \mathrm{H} 8 & 1.0000 & 0.11812 & 0.85985 & 0.53767 & \text { Biso } & 1.000 \mathrm{H}\end{array}$

\# Pd2.A2.T3; $E=-116.81 ; Z P V E=2.67 \mathrm{eV}$; frequencies= 1626.12i, 3061.89, 3061.86, 3027.74, 3008.43, 2987.80, 2972.84, 2814.73, $1484.77,1482.30,1474.42,1439.56,1389.20,1384.45,1313.91,1276.08,1205.11,1134.42,1088.92,1016.72,973.30,905.28,872.52$, $756.67,589.47,526.19,376.01,282.35,267.75,255.94,187.24,146.49,106.76,80.46,36.22,16.38 \mathrm{~cm}^{\wedge}-1$

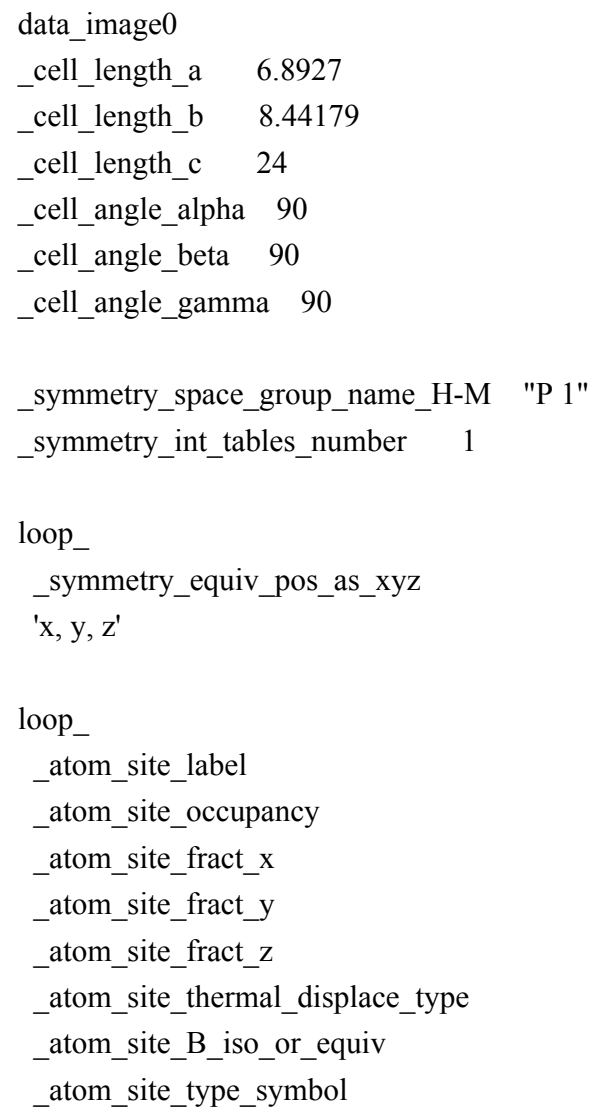




\begin{tabular}{|c|c|c|c|c|c|}
\hline Pd1 & 1.00000 .68941 & 0.16947 & 0.36817 & Biso & 1.000 \\
\hline $\mathrm{Pd} 2$ & 1.00000 .66658 & 0.50525 & 0.36681 & Biso & 1.000 \\
\hline $\mathrm{Pd} 3$ & 1.00000 .66751 & 0.82861 & 0.36666 & Biso & 1.000 \\
\hline $\mathrm{Pd} 4$ & 1.00000 .32824 & 0.99388 & 0.33974 & Biso & 1.000 \\
\hline $\mathrm{Pd} 5$ & 1.00000 .32818 & 0.33974 & 0.33998 & Biso & 1.000 \\
\hline Pd6 & 1.00000 .32904 & 0.66659 & 0.33835 & Biso & 1.000 \\
\hline d7 & 1.00000 .00336 & 0.16687 & 0.30651 & Biso & 1.000 \\
\hline Pd8 & 1.00000 .99882 & 0.50133 & 0.30691 & Biso & 1.000 \\
\hline Pd9 & 1.00000 .99958 & 0.83200 & 0.30664 & Biso & 1.000 \\
\hline $\operatorname{Pd} 10$ & 1.00000 .66993 & 0.00112 & 0.26849 & Biso & 1.000 \\
\hline Pd11 & 1.00000 .66964 & 0.33233 & 0.26864 & Biso & 1.000 \\
\hline Pd12 & 1.00000 .66643 & 0.66707 & 0.26717 & Biso & 1.000 \\
\hline d13 & 1.00000 .33406 & 0.16679 & 0.23670 & Biso & 1.000 \\
\hline Pd14 & 1.00000 .33183 & 0.49768 & 0.23817 & Biso & 1.000 \\
\hline Pd15 & 1.00000 .33213 & 0.83586 & 0.23815 & Biso & 1.000 \\
\hline Pd16 & 1.00000 .00189 & 0.00014 & 0.20329 & Biso & 1.000 \\
\hline Pd17 & 1.00000 .00178 & 0.33340 & 0.20327 & Biso & 1.000 \\
\hline Pd18 & 1.00000 .00333 & 0.66666 & 0.20350 & Biso & 1.000 \\
\hline Pd19 & 1.00000 .66897 & 0.16675 & 0.16784 & Biso & 1.000 \\
\hline $\operatorname{Pd} 20$ & 1.00000 .66897 & 0.50008 & 0.16784 & Biso & 1.000 \\
\hline $\operatorname{Pd} 21$ & 1.00000 .66897 & 0.83342 & 0.16784 & Biso & 1.00 \\
\hline $\mathrm{Pd} 22$ & 1.00000 .33838 & 0.00008 & 0.13524 & Biso & 1.000 \\
\hline $\operatorname{Pd} 23$ & 1.00000 .33838 & 0.33342 & 0.13524 & Biso & 1.000 \\
\hline $\operatorname{Pd} 24$ & 1.00000 .33838 & 0.66675 & 0.13524 & Biso & 1.000 \\
\hline $\operatorname{Pd} 25$ & 1.00000 .00202 & 0.16675 & 0.10321 & Biso & 1.000 \\
\hline $\operatorname{Pd} 26$ & 1.00000 .00202 & 0.50008 & 0.10321 & 1 Biso & 1.000 \\
\hline $\mathrm{Pd} 27$ & 1.00000 .00202 & 0.83342 & 0.10321 & Biso & 1.00 \\
\hline $\operatorname{Pd} 28$ & 1.00000 .66998 & 0.00008 & 0.06364 & 4 Biso & 1.00 \\
\hline $\operatorname{Pd} 29$ & 1.00000 .66998 & 0.33342 & 0.06364 & 4 Biso & 1.00 \\
\hline $\operatorname{Pd} 30$ & 1.00000 .66998 & 0.66675 & 0.06364 & 4 Biso & 1.000 \\
\hline $\mathrm{Pd} 31$ & 1.00000 .33931 & 0.16675 & 0.03394 & 4 Biso & 1.000 \\
\hline $\mathrm{Pd} 32$ & 1.00000 .33931 & 0.50008 & 0.03394 & 4 Biso & 1.000 \\
\hline Pd33 & 1.00000 .33931 & 0.83342 & 0.03394 & 4 Biso & 1.00 \\
\hline $\operatorname{Pd} 34$ & 1.00000 .00766 & 0.00008 & 0.00384 & 4 Biso & 1.00 \\
\hline $\operatorname{Pd} 35$ & 1.00000 .00766 & 0.33342 & 0.00384 & 4 Biso & 1.00 \\
\hline $\mathrm{Pd} 36$ & 1.00000 .00766 & 0.66675 & 0.00384 & 4 Biso & 1.00 \\
\hline $\mathrm{O} 1$ & 1.00000 .38623 & 0.16477 & 0.40135 & Biso & 1.000 \\
\hline $\mathrm{C} 1$ & 1.00000 .68410 & 0.98532 & 0.49190 & Biso & 1.000 \\
\hline $\mathrm{C} 2$ & 1.00000 .69944 & 0.14888 & 0.46544 & Biso & 1.000 \\
\hline $\mathrm{C} 3$ & 1.00000 .82926 & 0.96180 & 0.53975 & Biso & 1.000 \\
\hline H1 & 1.00000 .71269 & 0.89322 & 0.46008 & Biso & 1.000 \\
\hline $\mathrm{H} 2$ & 1.00000 .53577 & 0.96438 & 0.50654 & Biso & 1.000 \\
\hline H3 & 1.00000 .52435 & 0.15967 & 0.43345 & Biso & 1.000 \\
\hline H4 & 1.00000 .81473 & 0.84346 & 0.55780 & Biso & 1.000 \\
\hline H5 & 1.00000 .64687 & 0.24504 & 0.49217 & Biso & 1.000 \\
\hline H6 & 1.00000 .84995 & 0.17764 & 0.45436 & Biso & 1.000 \\
\hline H7 & 1.00000 .97830 & 0.97557 & 0.52483 & Biso & 1.000 \\
\hline H8 & 1.00000 .80508 & 0.04868 & 0.57220 & Biso & 1.000 \\
\hline
\end{tabular}

\# Pd2.A2.1p; $E=-118.08 ; \mathrm{ZPVE}=2.83 \mathrm{eV}$; frequencies=3738.12, 3070.65, 3052.66, 3043.16, 3021.92, 2986.51, 2985.83, 2967.13, 1496.71, 1478.21, 1475.67, 1442.71, 1385.10, 1309.51, 1303.32, 1207.95, 1119.36, 1046.61, 983.27, 951.83, 890.33, 788.26, 651.66, 593.71, 544.31, $449.84,329.94,248.91,239.75,227.38,178.60,168.72,103.76,79.59,62.63,27.19 \mathrm{~cm}^{\wedge}-1$ 


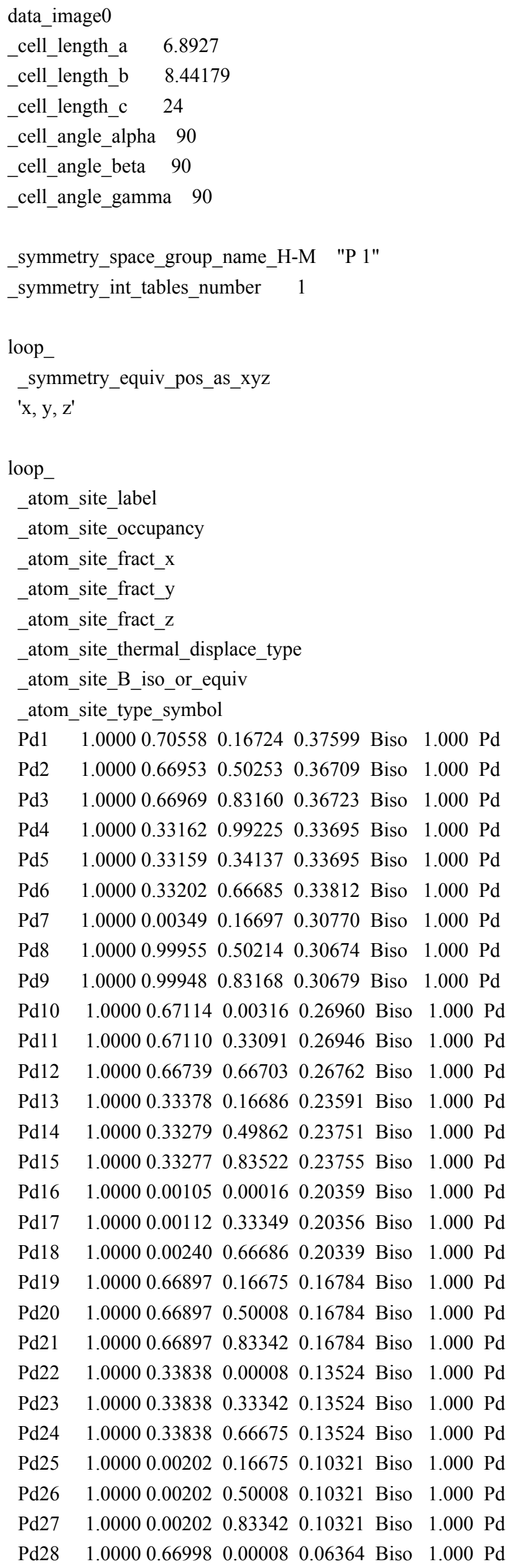




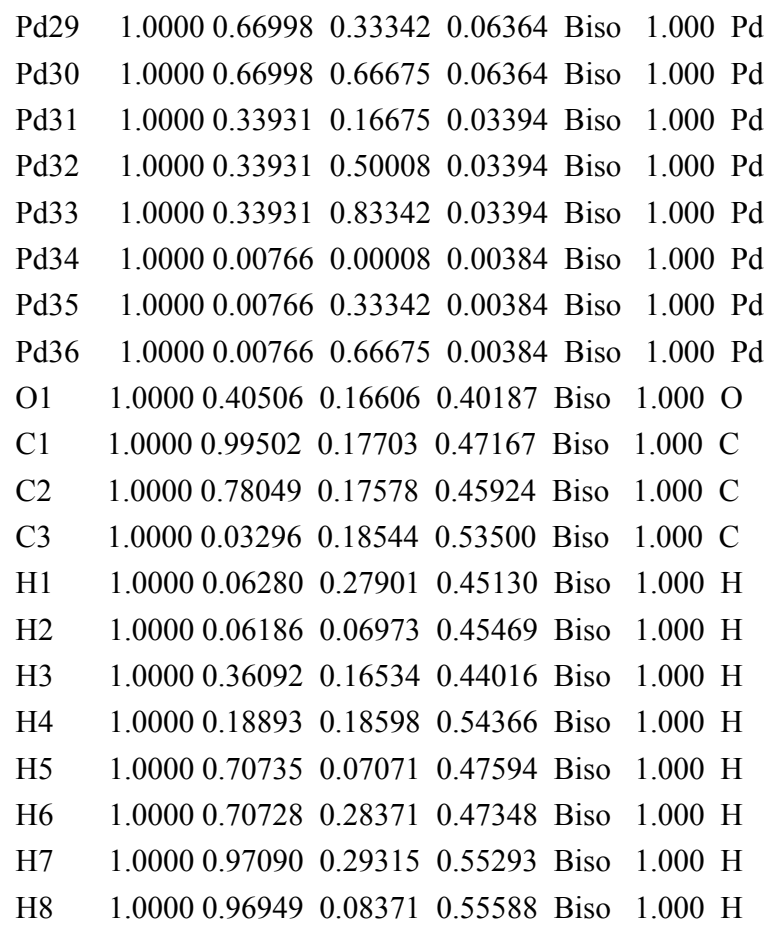

\# Pd2.A2.T4; $E=-117.48 ; \mathrm{ZPVE}=2.65 \mathrm{eV}$; frequencies=1420.65i, 3714.65, 3058.13, 3055.28, 3022.46, 2995.58, 2967.72, 2963.13, $1484.84,1473.00,1453.13,1431.09,1386.48,1348.10,1276.57,1185.15,1145.35,1047.28,969.27,957.78,897.06,876.50,784.22,640.81$, $536.96,409.18,365.20,344.63,259.82,199.05,176.86,136.37,73.90,67.87,52.21,32.14 \mathrm{~cm}^{\wedge}-1$

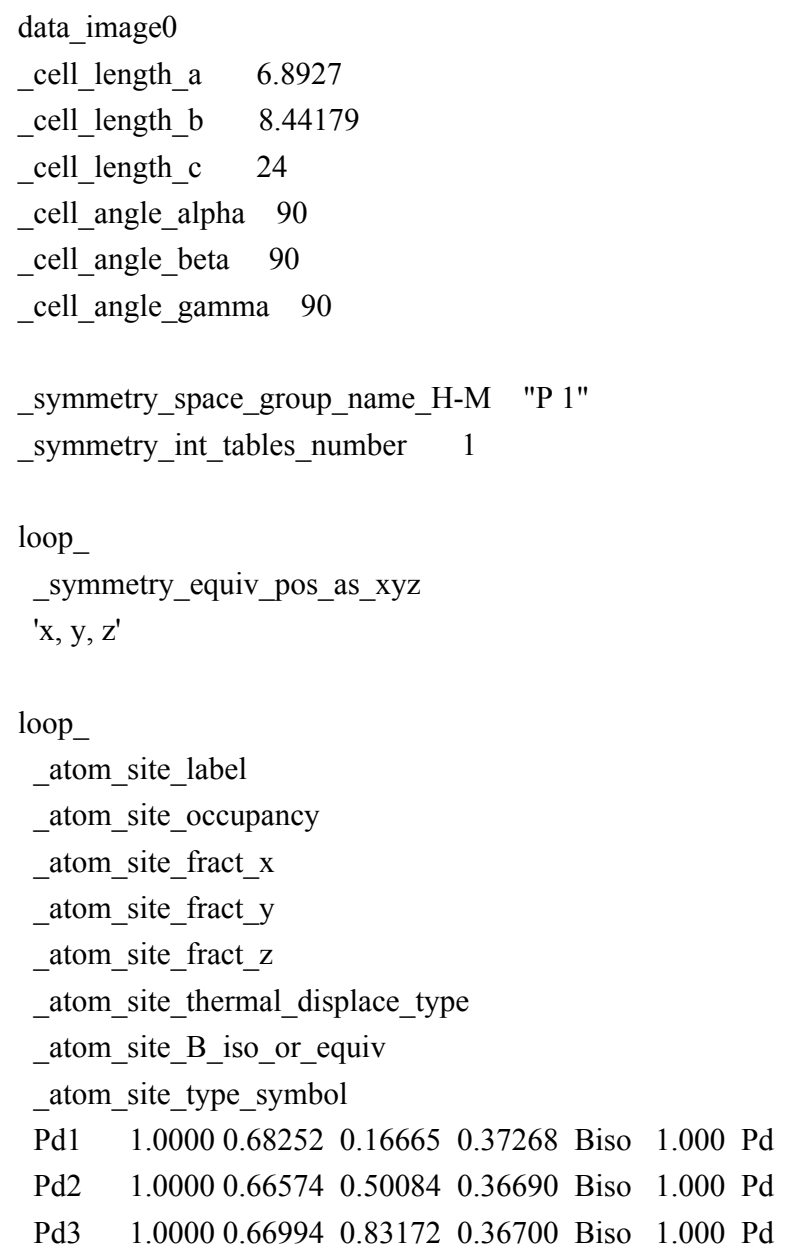




\begin{tabular}{|c|c|c|c|c|c|}
\hline $\operatorname{Pd} 4$ & 1.00000 .33842 & 0.99908 & 0.33740 & Biso & 1.000 \\
\hline Pd5 & 1.00000 .32820 & 0.32983 & 0.34204 & Biso & 1.000 \\
\hline Pd6 & 1.00000 .33550 & 0.66739 & 0.33694 & Biso & 1.000 \\
\hline $\mathrm{Pd} 7$ & 1.00000 .00276 & 0.16505 & 0.30667 & Biso & 1.000 \\
\hline $\operatorname{Pd} 8$ & 1.00000 .00127 & 0.50081 & 0.30661 & Biso & 1.000 \\
\hline Pd9 & 1.00000 .00537 & 0.83271 & 0.30725 & Biso & 1.000 \\
\hline Pd10 & 1.00000 .67047 & 0.00143 & 0.26903 & 3 Biso & 1.00 \\
\hline Pd11 & 1.00000 .66405 & 0.33155 & 0.27004 & 4 Biso & 1.000 \\
\hline Pd12 & 1.00000 .67070 & 0.66674 & 0.26764 & 4 Biso & 1.000 \\
\hline Pd13 & 1.00000 .33134 & 0.16785 & 0.23689 & 7 Biso & 1.000 \\
\hline Pd14 & 1.00000 .33244 & 0.49788 & 0.237 & Biso & \\
\hline Pd15 & 1.00000 .33490 & 0.83385 & 0.23595 & Biso & 1.000 \\
\hline d16 & 1.00000 .00144 & 0.99973 & 0.20339 & Biso & 1.00 \\
\hline Pd17 & 1.00000 .99859 & 0.33328 & 0.20354 & 4 Biso & 1.000 \\
\hline Pd18 & 1.00000 .00306 & 0.66662 & 0.20329 & Biso & 1.000 \\
\hline Pd19 & 1.00000 .66897 & 0.16675 & 0.16784 & 4 Biso & 1.000 \\
\hline $\operatorname{Pd} 20$ & 1.00000 .66897 & 0.50008 & 0.16784 & 4 Biso & 1.000 \\
\hline d21 & 1.00000 .66897 & 0.83342 & 0.16784 & 4 Biso & 1.000 \\
\hline d22 & 1.00000 .33838 & 0.00008 & 0.13524 & 4 Biso & 1.00 \\
\hline $\operatorname{Pd} 23$ & 1.00000 .33838 & 0.33342 & 0.13524 & 4 Biso & 1.00 \\
\hline $\operatorname{Pd} 24$ & 1.00000 .33838 & 0.66675 & 0.13524 & 4 Biso & 1.000 \\
\hline $\operatorname{Pd} 25$ & 1.00000 .00202 & 0.16675 & 0.10321 & Biso & 1.000 \\
\hline $\operatorname{Pd} 26$ & 1.00000 .00202 & 0.50008 & 0.10321 & Biso & 1.000 \\
\hline $\operatorname{Pd} 27$ & 1.00000 .00202 & 0.83342 & 0.10321 & Biso & 1.000 \\
\hline $\mathrm{Pd} 28$ & 1.00000 .66998 & 0.00008 & 0.06364 & 4 Biso & 1.000 \\
\hline $\operatorname{Pd} 29$ & 1.00000 .66998 & 0.33342 & 0.06364 & 4 Biso & 1.00 \\
\hline $\operatorname{Pd} 30$ & 1.00000 .66998 & 0.66675 & 0.06364 & 4 Biso & 1.00 \\
\hline $\mathrm{Pd} 31$ & 1.00000 .33931 & 0.16675 & 0.03394 & 4 Biso & 1.00 \\
\hline $\operatorname{Pd} 32$ & 1.00000 .33931 & 0.50008 & 0.03394 & 4 Biso & 1.000 \\
\hline Pd33 & 1.00000 .33931 & 0.83342 & 0.03394 & 4 Biso & 1.000 \\
\hline $\operatorname{Pd} 34$ & 1.00000 .00766 & 0.00008 & 0.00384 & 4 Biso & 1.00 \\
\hline Pd35 & 1.00000 .00766 & 0.33342 & 0.00384 & 4 Biso & 1.00 \\
\hline Pd36 & 1.00000 .00766 & 0.66675 & 0.00384 & 4 Biso & 1.000 \\
\hline $\mathrm{O} 1$ & 1.00000 .19647 & 0.32786 & 0.42333 & Biso & 1.000 \\
\hline $\mathrm{C} 1$ & 1.00000 .89977 & 0.23690 & 0.48199 & Biso & 1.000 \\
\hline $\mathrm{C} 2$ & 1.00000 .73607 & 0.15124 & 0.45985 & Biso & 1.000 \\
\hline $\mathrm{C} 3$ & 1.00000 .85835 & 0.39462 & 0.51067 & Biso & 1.000 \\
\hline H1 & 1.00000 .03559 & 0.27449 & 0.44500 & Biso & 1.000 \\
\hline $\mathrm{H} 2$ & 1.00000 .99947 & 0.16006 & 0.50517 & Biso & 1.000 \\
\hline H3 & 1.00000 .19304 & 0.43933 & 0.43343 & Biso & 1.000 \\
\hline H4 & 1.00000 .99233 & 0.45455 & 0.52288 & Biso & 1.000 \\
\hline H5 & 1.00000 .74403 & 0.02219 & 0.46269 & Biso & 1.000 \\
\hline H6 & 1.00000 .59123 & 0.19993 & 0.46803 & Biso & 1.000 \\
\hline H7 & 1.00000 .77781 & 0.47437 & 0.48303 & Biso & 1.000 \\
\hline H8 & 1.00000 .77091 & 0.37778 & 0.54773 & Biso & 1.000 \\
\hline
\end{tabular}

\# Pd2.A2.y; $E=-119.17 ; \mathrm{ZPVE}=2.77 \mathrm{eV}$; frequencies=18.53i, 3825.57, 3649.63, 3166.27, 3082.86, 3072.54, 3052.37, 3024.57, 2965.24, 1607.39, 1520.30, 1471.99, 1459.14, 1401.06, 1386.79, 1242.92, 1171.14, 1033.32, 964.90, 914.61, 910.80, 883.07, 689.74, 407.72, 351.69, $270.78,266.66,196.20,160.88,150.42,94.93,75.94,54.31,49.75,43.62,17.78 \mathrm{~cm}^{\wedge}-1$

data_image 0

_cell_length_a 6.8927 


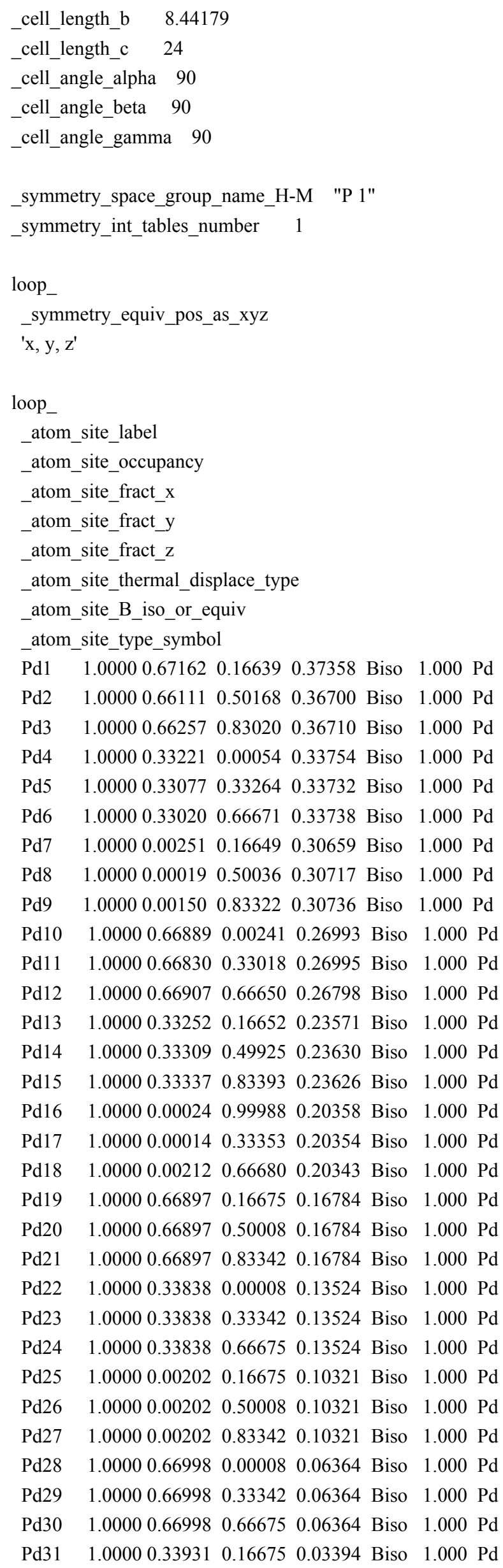




$\begin{array}{llllllll}\text { Pd32 } & 1.0000 & 0.33931 & 0.50008 & 0.03394 & \text { Biso } & 1.000 \mathrm{Pd} \\ \text { Pd33 } & 1.0000 & 0.33931 & 0.83342 & 0.03394 & \text { Biso } & 1.000 \mathrm{Pd} \\ \text { Pd34 } & 1.0000 & 0.00766 & 0.00008 & 0.00384 & \text { Biso } & 1.000 \mathrm{Pd} \\ \text { Pd35 } & 1.0000 & 0.00766 & 0.33342 & 0.00384 & \text { Biso } & 1.000 \mathrm{Pd} \\ \text { Pd36 } & 1.0000 & 0.00766 & 0.66675 & 0.00384 & \text { Biso } & 1.000 \mathrm{Pd} \\ \mathrm{O} 1 & 1.0000 & 0.03292 & 0.60333 & 0.46512 & \text { Biso } & 1.000 & \mathrm{O} \\ \mathrm{C} 1 & 1.0000 & 0.76600 & 0.10134 & 0.46080 & \text { Biso } & 1.000 \mathrm{C} \\ \mathrm{C} 2 & 1.0000 & 0.62418 & 0.21878 & 0.46283 & \text { Biso } & 1.000 \mathrm{C} \\ \mathrm{C} 3 & 1.0000 & 0.97884 & 0.13157 & 0.46980 & \text { Biso } & 1.000 \mathrm{C} \\ \mathrm{H} 1 & 1.0000 & 0.15259 & 0.64209 & 0.48109 & \text { Biso } & 1.000 \mathrm{H} \\ \mathrm{H} 2 & 1.0000 & 0.72011 & 0.97852 & 0.46585 & \text { Biso } & 1.000 \mathrm{H} \\ \mathrm{H} 3 & 1.0000 & 0.05415 & 0.60960 & 0.42512 & \text { Biso } & 1.000 \mathrm{H} \\ \mathrm{H} 4 & 1.0000 & 0.06817 & 0.05730 & 0.44266 & \text { Biso } & 1.000 \mathrm{H} \\ \mathrm{H} 5 & 1.0000 & 0.47300 & 0.18855 & 0.46966 & \text { Biso } & 1.000 \mathrm{H} \\ \mathrm{H} 6 & 1.0000 & 0.66501 & 0.34077 & 0.47139 & \text { Biso } & 1.000 \mathrm{H} \\ \mathrm{H} 7 & 1.0000 & 0.01659 & 0.25573 & 0.46304 & \text { Biso } & 1.000 \mathrm{H} \\ \mathrm{H} 8 & 1.0000 & 0.01595 & 0.10048 & 0.51235 & \text { Biso } & 1.000 \mathrm{H}\end{array}$

\# Pd2.B2.P; $E=-118.44 ; \mathrm{ZPVE}=2.84 \mathrm{eV}$; frequencies= 8.87i, 3066.17, 3051.70, 3047.44, 3030.43, 2989.54, 2984.41, 2966.46, 2848.76, $1492.80,1487.29,1478.52,1466.92,1449.08,1401.99,1381.30,1335.72,1283.80,1192.70,1156.85,1044.42,919.20,900.70,857.13$, $746.39,408.77,369.16,348.33,344.18,268.37,219.64,79.34,64.95,47.12,26.21,21.64 \mathrm{~cm}^{\wedge}-1$

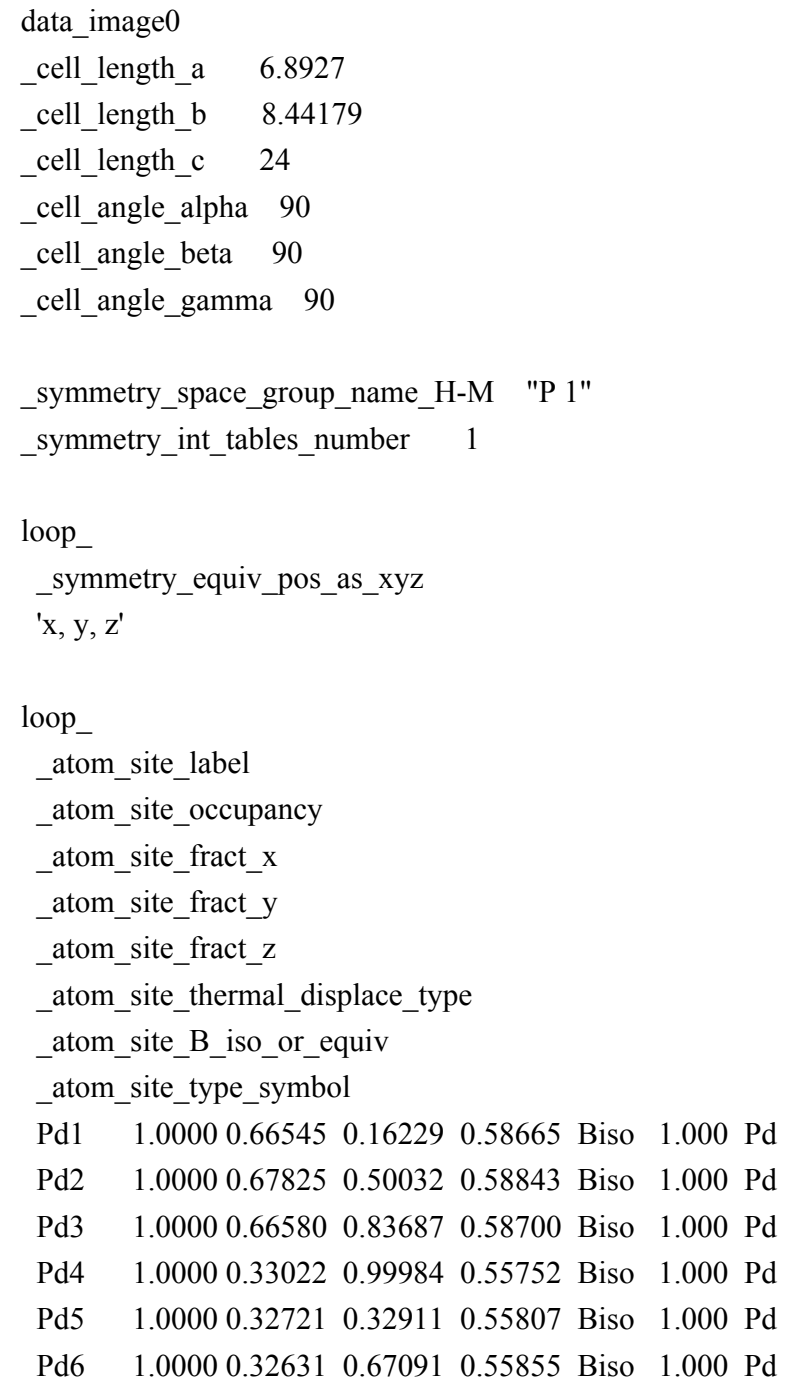




\begin{tabular}{|c|c|c|c|}
\hline $\operatorname{Pd} 7$ & 1.00000 .998430 .16566 & 0.52625 Biso & 1.000 \\
\hline Pd8 & 1.00000 .996190 .50007 & 0.52522 Biso & 1.000 \\
\hline d9 & $\begin{array}{lll}1.0000 & 0.99811 & 0.83422\end{array}$ & 0.52658 Biso & 1.000 \\
\hline $\operatorname{Pd} 10$ & $\begin{array}{lll}1.0000 & 0.66637 & 0.99979\end{array}$ & 0.48684 Biso & 1.000 \\
\hline d11 & $\begin{array}{lll}1.0000 & 0.66950 & 0.33292\end{array}$ & 20.48729 Biso & 1.000 \\
\hline Pd12 & $\begin{array}{lll}1.0000 & 0.66900 & 0.66711\end{array}$ & 0.48742 Biso & 1.000 \\
\hline $\operatorname{Pd} 13$ & $1.0000 \quad 0.33158 \quad 0.16855$ & 50.45712 Biso & \\
\hline Pd14 & $1.0000 \quad 0.334250 .50008$ & 0.45509 Biso & 1.000 \\
\hline Pd15 & 331210.83127 & 70.45724 Biso & 1.0 \\
\hline Pd16 & 1.00000 .002470 .99993 & 0.42272 Biso & 1.000 \\
\hline Pd17 & $\begin{array}{lll}1.0000 & 0.00198 & 0.33323\end{array}$ & 30.42246 Biso & 1.00 \\
\hline $\operatorname{Pd} 18$ & 1.00000 .001690 .66689 & 9.42251 Biso & 1.00 \\
\hline d19 & 1.00000 .668970 .16673 & 30.38692 Biso & 1.00 \\
\hline $\operatorname{Pd} 20$ & 1.00000 .668970 .50006 & 60.38692 Biso & 1.00 \\
\hline $\operatorname{Pd} 21$ & 1.00000 .668970 .83340 & 0.38692 Biso & 1.00 \\
\hline $\operatorname{Pd} 22$ & 1.00000 .338380 .00006 & 60.35432 Biso & 1.00 \\
\hline $\operatorname{Pd} 23$ & $\begin{array}{lll}1.0000 & 0.33838 & 0.33340\end{array}$ & 0.35432 Biso & 1.00 \\
\hline $\mathrm{Pd} 24$ & $1.0000 \quad 0.33838 \quad 0.66673$ & 30.35432 Biso & 1.000 \\
\hline $\operatorname{Pd} 25$ & 1.00000 .002020 .16673 & 3 0.32230 Biso & 1.000 \\
\hline Pd26 & 1.00000 .002020 .50006 & 60.32230 Biso & 1.00 \\
\hline $\operatorname{Pd} 27$ & 1.00000 .002020 .83340 & 0.32230 Biso & 1.00 \\
\hline $\operatorname{Pd} 28$ & 1.00000 .669980 .00006 & 60.28273 Biso & 1.00 \\
\hline $\operatorname{Pd} 29$ & $\begin{array}{lll}1.0000 & 0.66998 & 0.33340\end{array}$ & 0.28273 Biso & 1.000 \\
\hline $\operatorname{Pd} 30$ & $1.00000 .66998 \quad 0.66673$ & 30.28273 Biso & 1.000 \\
\hline $\mathrm{Pd} 31$ & $\begin{array}{lll}1.0000 & 0.33931 & 0.16673\end{array}$ & 0.25303 Biso & 1.000 \\
\hline $\mathrm{Pd} 32$ & 1.00000 .339310 .50006 & 60.25303 Biso & 1.000 \\
\hline $\operatorname{Pd} 33$ & $\begin{array}{lll}1.0000 & 0.33931 & 0.83340\end{array}$ & 0.25303 Biso & 1.00 \\
\hline $\mathrm{Pd} 34$ & 1.00000 .007660 .00006 & 60.22292 Biso & 1.00 \\
\hline $\mathrm{Pd} 35$ & $\begin{array}{lll}1.0000 & 0.00766 & 0.33340\end{array}$ & 0.22292 Biso & 1.00 \\
\hline Pd36 & 1.00000 .007660 .66673 & 30.22292 Biso & 1.000 \\
\hline $\mathrm{O} 1$ & $\begin{array}{lll}1.0000 & 0.39732 & 0.49967\end{array}$ & 0.61318 Biso & 1.000 \\
\hline $\mathrm{C} 1$ & $\begin{array}{lll}1.0000 & 0.94710 & 0.41482\end{array}$ & 0.70717 Biso & 1.000 \\
\hline $\mathrm{C} 2$ & 1.00000 .084820 .53480 & 0.73396 Biso & 1.000 \\
\hline $\mathrm{C} 3$ & $\begin{array}{lll}1.0000 & 0.02951 & 0.24655\end{array}$ & 0.70539 Biso & 1.000 \\
\hline H1 & 1.00000 .915070 .45360 & 0.66415 Biso & 1.000 \\
\hline $\mathrm{H} 2$ & $1.0000 \quad 0.807870 .41456$ & 0.72930 Biso & 1.000 \\
\hline H3 & $\begin{array}{lll}1.0000 & 0.02145 & 0.65363\end{array}$ & 0.73442 Biso & 1.000 \\
\hline $\mathrm{H} 4$ & $\begin{array}{lll}1.0000 & 0.92902 & 0.16399\end{array}$ & 0.68514 Biso & 1.000 \\
\hline H5 & 1.00000 .116970 .50171 & 0.77709 Biso & 1.000 \\
\hline H6 & $\begin{array}{lll}1.0000 & 0.22193 & 0.54061\end{array}$ & 0.71113 Biso & 1.000 \\
\hline H7 & $\begin{array}{lll}1.0000 & 0.16625 & 0.24299\end{array}$ & 0.68223 Biso & 1.000 \\
\hline H8 & $\begin{array}{lll}1.0000 & 0.05755 & 0.20217\end{array}$ & 0.74687 Biso & 1.000 \\
\hline
\end{tabular}

\# Pd2.B2.T3; $E=-116.71 ; Z P V E=2.66 \mathrm{eV}$; frequencies=1701.48i, 3056.81, 3055.24, 2990.75, 2982.16, 2939.71, 2933.46, 2911.06, 1513.63, 1472.07, 1468.40, 1465.22, 1449.98, 1393.09, 1376.60, 1311.14, 1167.88, 1126.59, 1092.96, 1000.08, 926.31, 921.04, 901.26, $855.69,467.34,372.67,302.93,268.70,259.05,242.08,231.41,163.19,142.46,63.61,45.35,21.23 \mathrm{~cm}^{\wedge}-1$

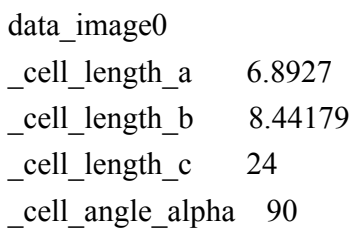




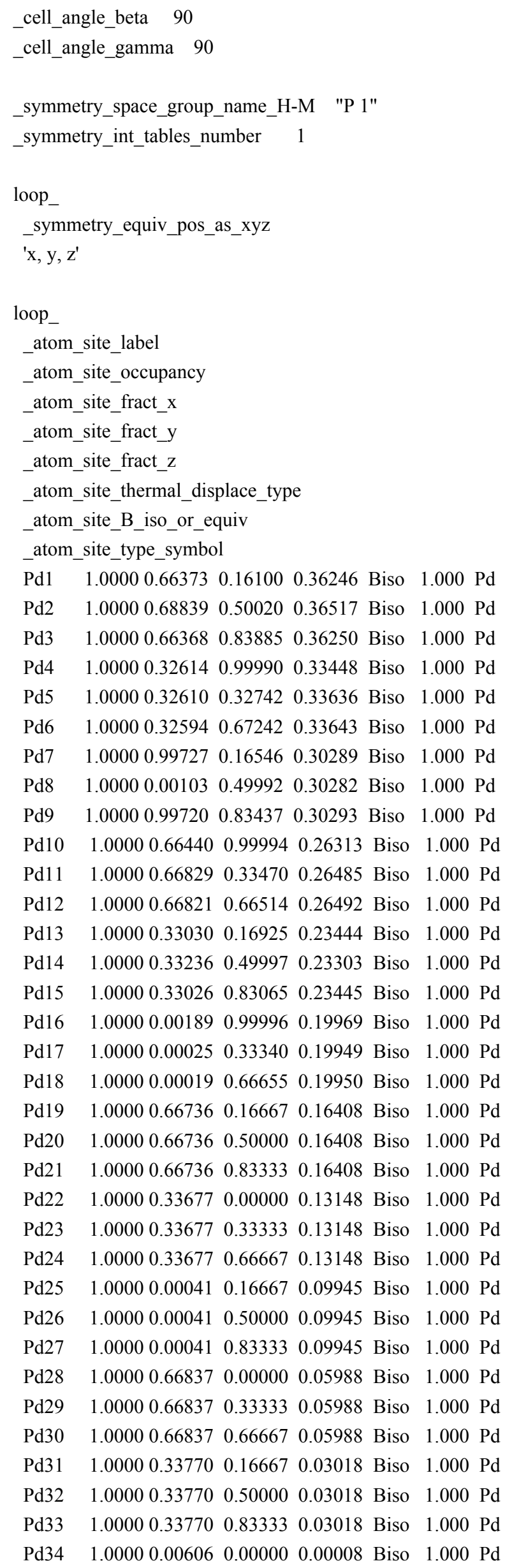




$\begin{array}{llllllll}\text { Pd35 } & 1.0000 & 0.00606 & 0.33333 & 0.00008 & \text { Biso } & 1.000 \mathrm{Pd} \\ \mathrm{Pd} 36 & 1.0000 & 0.00606 & 0.66667 & 0.00008 & \text { Biso } & 1.000 \mathrm{Pd} \\ \mathrm{O} 1 & 1.0000 & 0.39001 & 0.49966 & 0.39754 & \text { Biso } & 1.000 \mathrm{O} \\ \mathrm{C} 1 & 1.0000 & 0.69970 & 0.49741 & 0.46663 & \text { Biso } & 1.000 \mathrm{C} \\ \mathrm{C} 2 & 1.0000 & 0.81193 & 0.64987 & 0.47735 & \text { Biso } & 1.000 \mathrm{C} \\ \mathrm{C} 3 & 1.0000 & 0.81724 & 0.34682 & 0.47583 & \text { Biso } & 1.000 \mathrm{C} \\ \mathrm{H} 1 & 1.0000 & 0.53559 & 0.49842 & 0.42991 & \text { Biso } & 1.000 \mathrm{H} \\ \mathrm{H} 2 & 1.0000 & 0.57305 & 0.49465 & 0.49483 & \text { Biso } & 1.000 \mathrm{H} \\ \mathrm{H} 3 & 1.0000 & 0.72453 & 0.75547 & 0.46851 & \text { Biso } & 1.000 \mathrm{H} \\ \mathrm{H} 4 & 1.0000 & 0.73375 & 0.23986 & 0.46579 & \text { Biso } & 1.000 \mathrm{H} \\ \mathrm{H} 5 & 1.0000 & 0.85353 & 0.65636 & 0.52157 & \text { Biso } & 1.000 \mathrm{H} \\ \mathrm{H} 6 & 1.0000 & 0.94514 & 0.65612 & 0.45276 & \text { Biso } & 1.000 \mathrm{H} \\ \mathrm{H} 7 & 1.0000 & 0.95083 & 0.34575 & 0.45132 & \text { Biso } & 1.000 \mathrm{H} \\ \mathrm{H} 8 & 1.0000 & 0.85854 & 0.33741 & 0.51938 & \text { Biso } & 1.000 \mathrm{H}\end{array}$

\# Pd2.B2.2p; $E=-118.18 ; \mathrm{ZPVE}=2.79 \mathrm{eV}$; frequencies=3724.42, 3081.95, 3078.01, 2966.65, 2951.53, 2932.42, 2918.64, 2907.29, 1466.42, 1452.92, 1451.03, 1433.48, 1379.72, 1364.83, 1306.47, 1183.10, 1092.98, 1079.59, 912.60, 908.39, 882.95, 854.64, 597.38, 584.80, 424.62, $382.69,321.98,236.94,216.38,194.60,187.57,181.73,173.28,75.18,59.40,43.43 \mathrm{~cm}^{\wedge}-1$

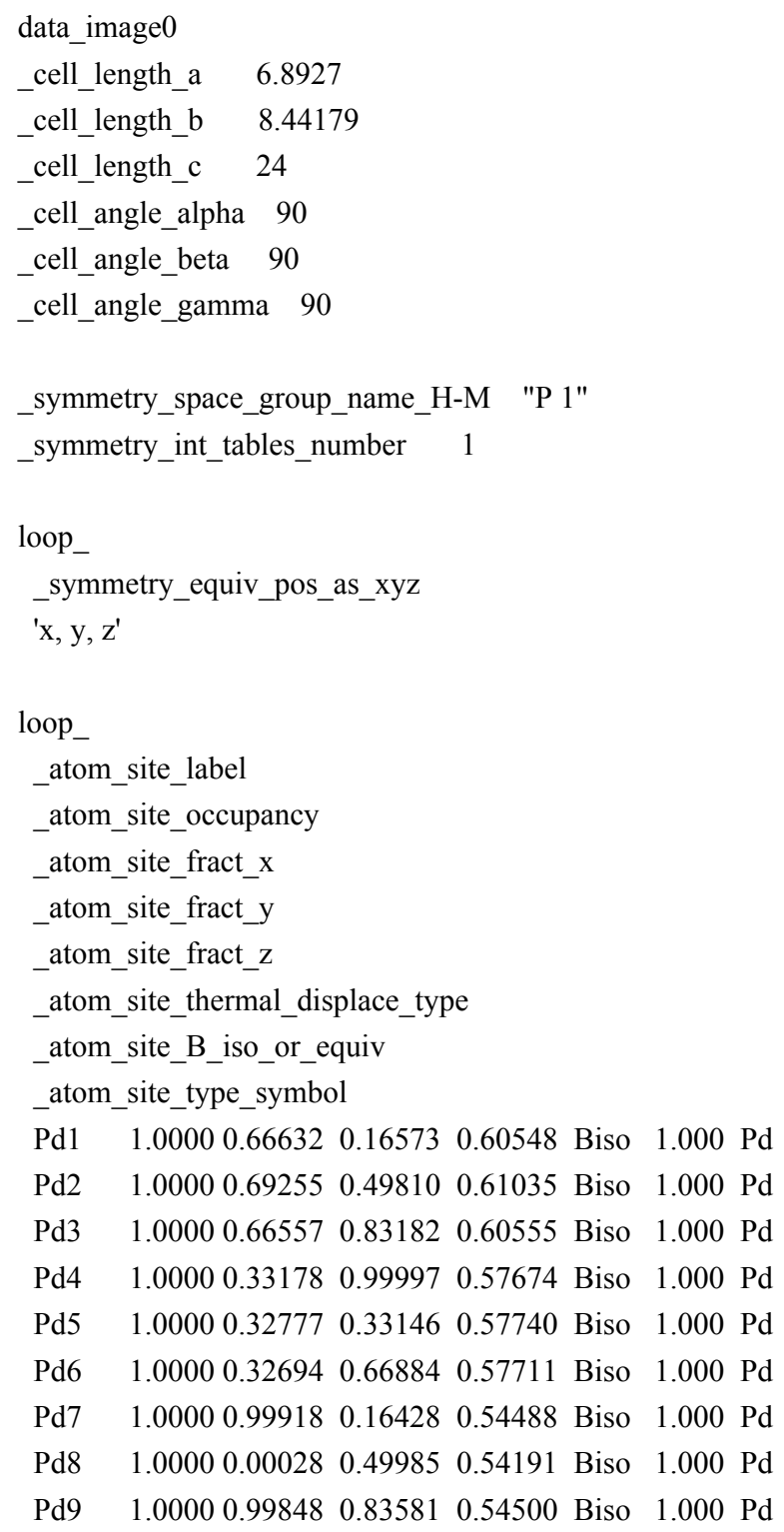




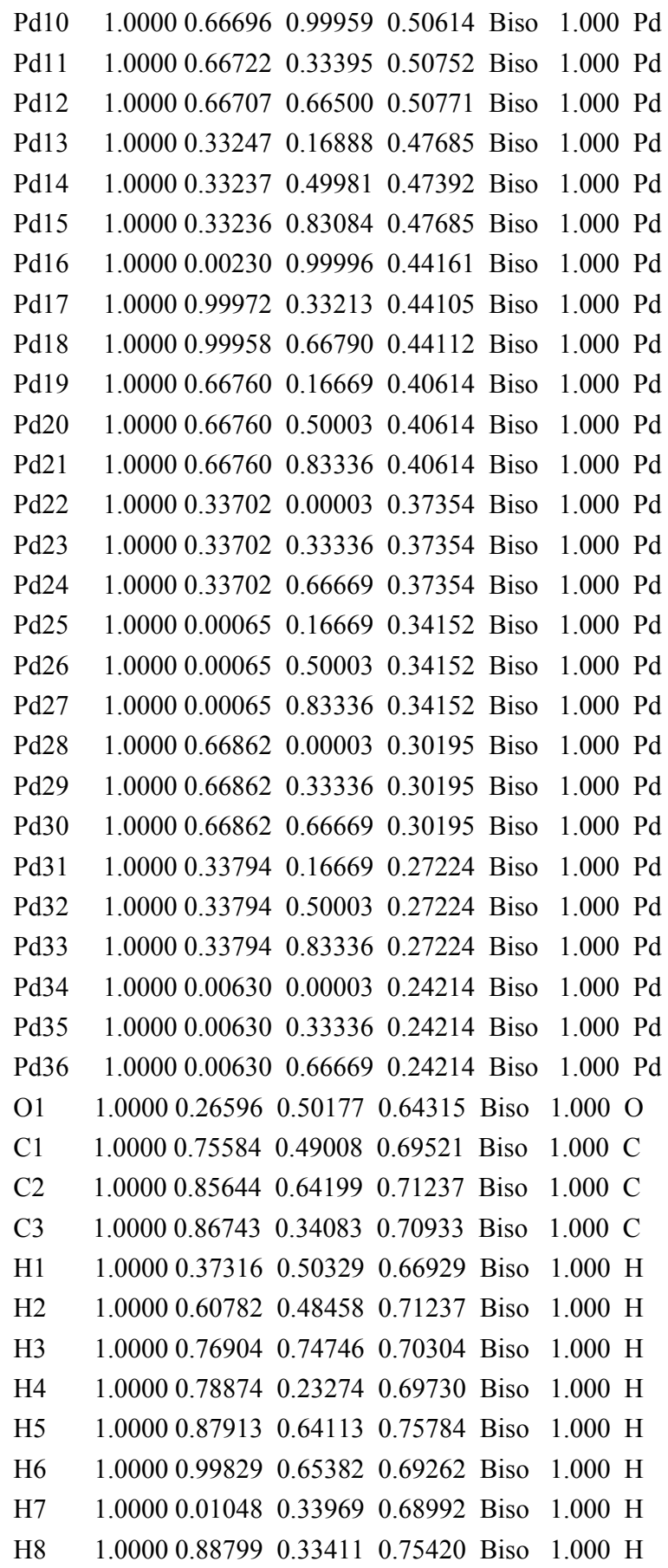

\# Pd2.B2.T4; $\mathrm{E}=-117.52 ; \mathrm{ZPVE}=2.64 \mathrm{eV}$; frequencies $=1474.91 \mathrm{i}, 3708.10,3089.24,3052.88,2993.06,2969.32,2953.91,2919.97$, $1503.18,1460.95,1447.78,1430.68,1374.09,1349.99,1301.00,1176.66,1146.80,1019.88,989.53,951.05,892.12,851.27,829.68,556.24$, $517.30,409.86,368.48,335.35,224.67,189.19,181.77,166.94,89.92,76.09,49.69,46.10 \mathrm{~cm}^{\wedge}-1$

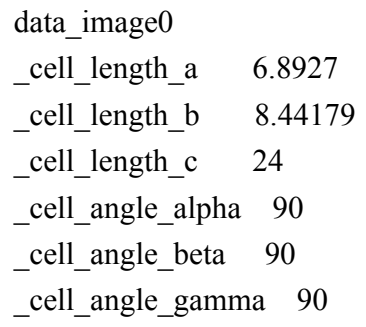




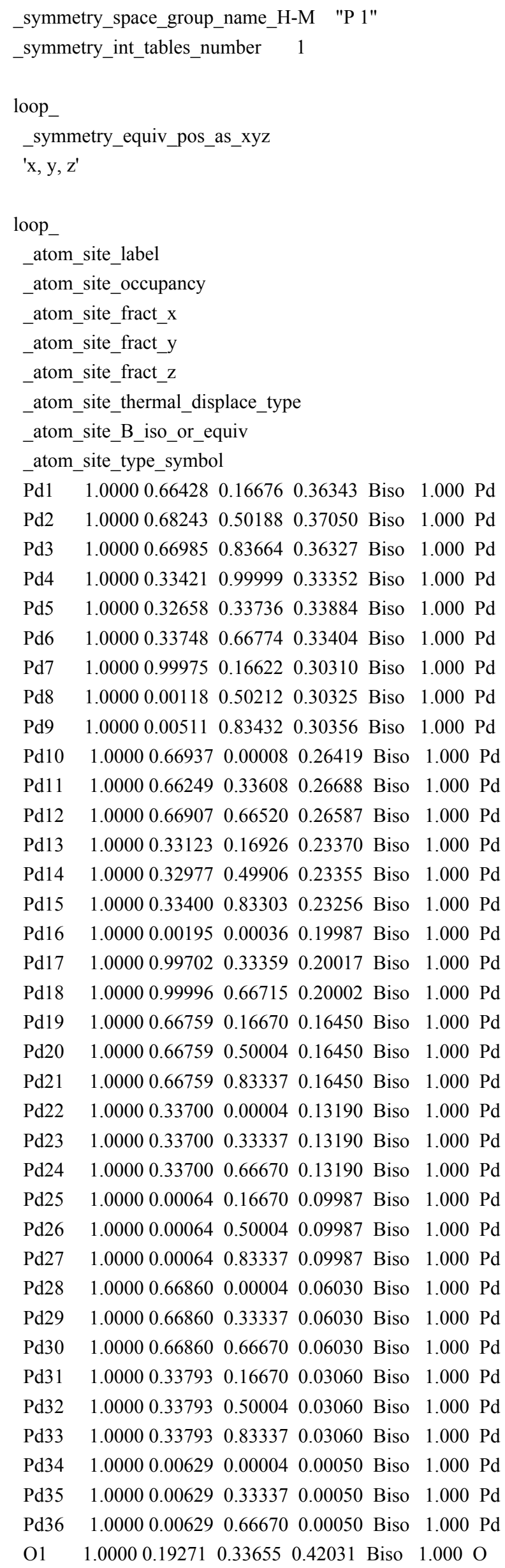


C1 $\quad 1.00000 .72541 \quad 0.50670 \quad 0.45943$ Biso $1.000 \mathrm{C}$

$\begin{array}{lllllllll}\mathrm{C} 2 & 1.0000 & 0.75603 & 0.67551 & 0.47837 & \text { Biso } & 1.000 \mathrm{C}\end{array}$

$\begin{array}{llllllll}\mathrm{C} 3 & 1.0000 & 0.87171 & 0.39203 & 0.47386 & \text { Biso } & 1.000 \mathrm{C}\end{array}$

H1 $1.0000 \quad 0.20438 \quad 0.22656 \quad 0.43187$ Biso $1.000 \mathrm{H}$

$\begin{array}{llllllll}\mathrm{H} 2 & 1.0000 & 0.57385 & 0.46580 & 0.46052 & \text { Biso } & 1.000 \mathrm{H}\end{array}$

H3 1.00000 .652960 .758660 .45922 Biso $1.000 \mathrm{H}$

H4 $1.00000 .81890 \quad 0.271200 .47915$ Biso $1.000 \mathrm{H}$

H5 1.00000 .732480 .683210 .52363 Biso $1.000 \mathrm{H}$

H6 $1.00000 .90398 \quad 0.715550 .46989$ Biso $1.000 \mathrm{H}$

H7 $1.0000 \quad 0.02303 \quad 0.372740 .43901$ Biso $1.000 \mathrm{H}$

H8 1.00000 .967140 .429750 .50723 Biso $1.000 \mathrm{H}$

\# Pd2.B2.y; $E=-119.18 ; \mathrm{ZPVE}=2.78 \mathrm{eV}$; frequencies= 3820.76, 3617.25, 3167.97, 3096.16, 3072.60, 3059.13, 3031.81, 2968.14, 1608.71, 1520.26, 1472.62, 1461.82, 1404.21, 1388.81, 1249.10, 1172.91, 1034.31, 970.06, 933.59, 913.36, 883.51, 706.79, 412.52, 358.00, 267.11, $250.38,181.83,161.15,149.92,113.79,87.17,74.28,62.44,48.43,37.77,24.72 \mathrm{~cm}^{\wedge}-1$

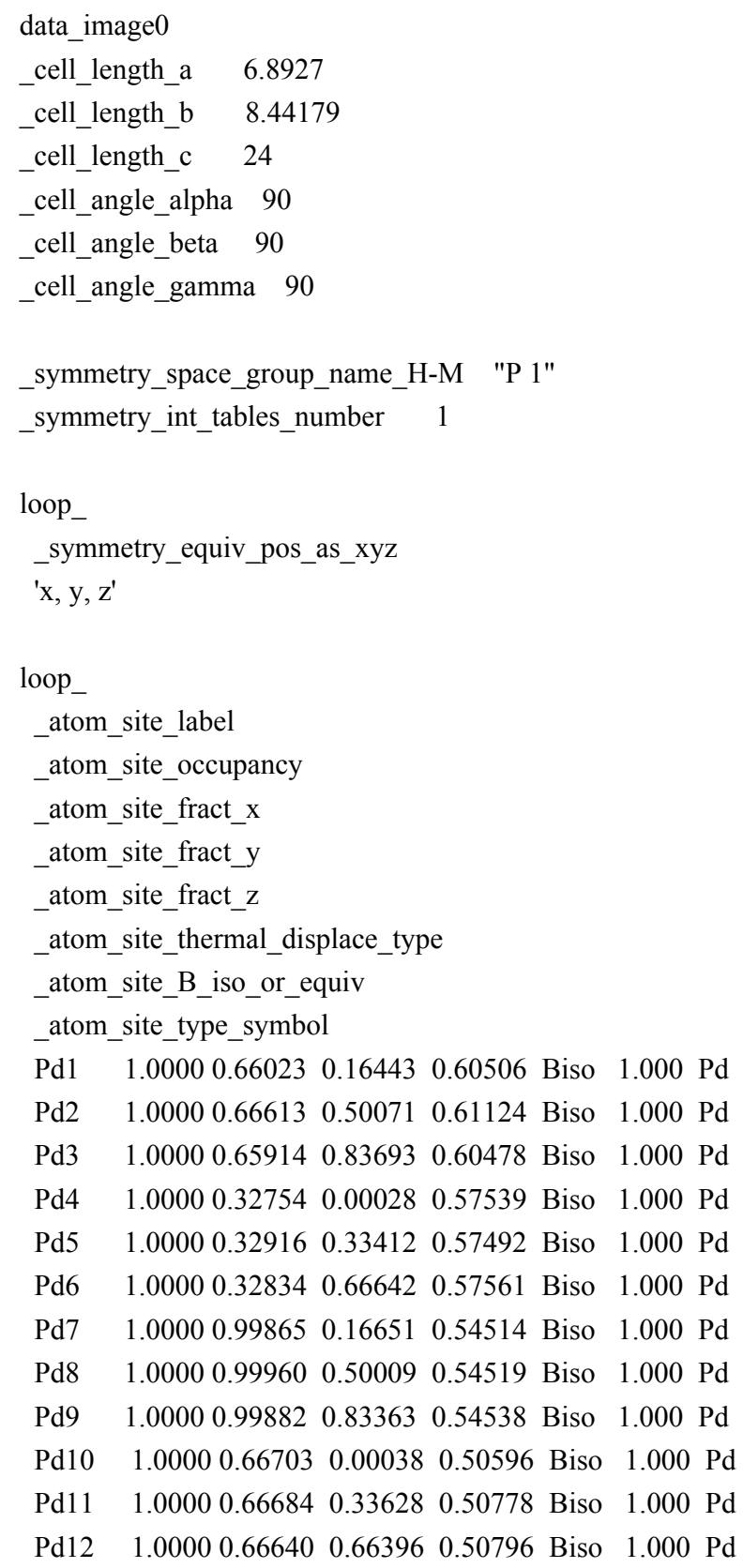




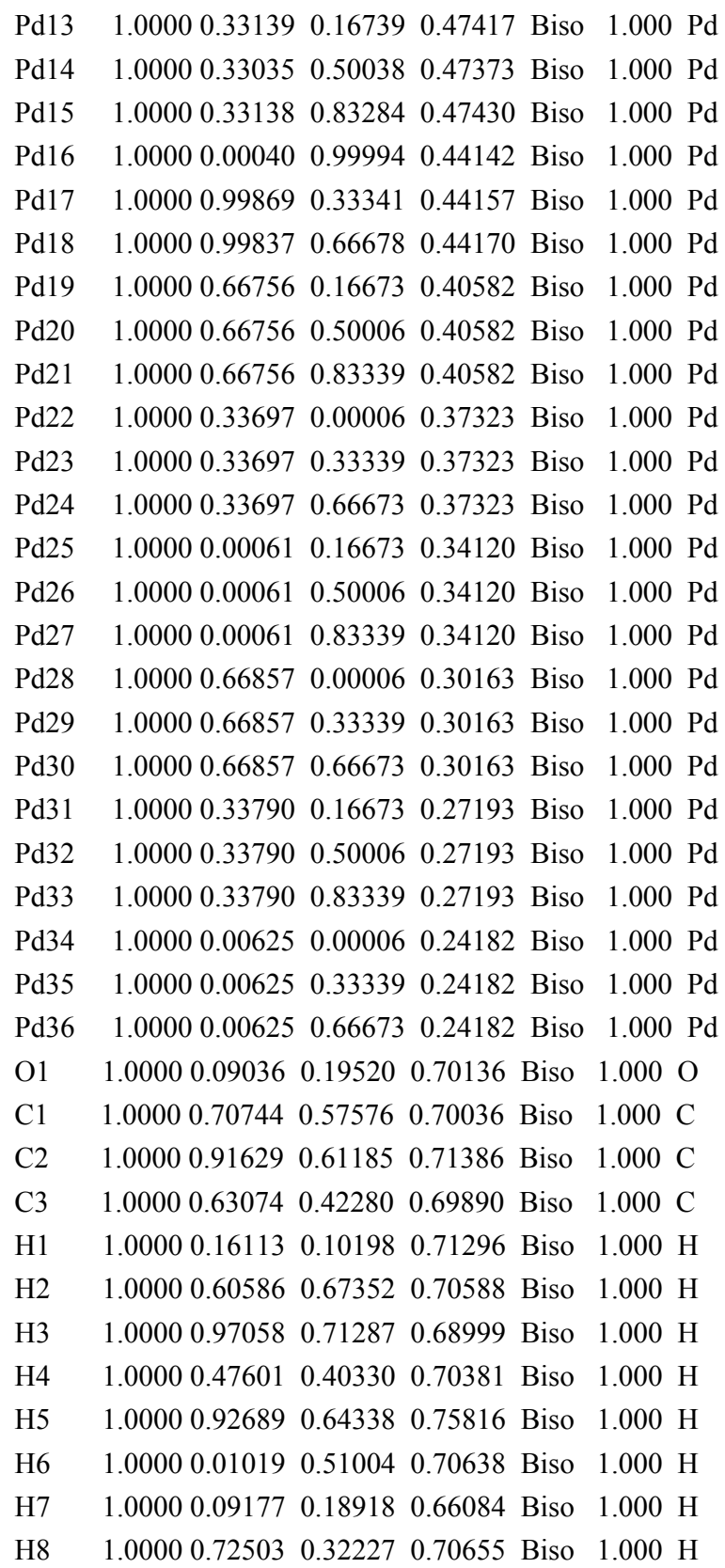

\# Pd1.C1.P; E= -123.09; ZPVE= 3.12 eV; frequencies= 16.16i, 3698.64, 3056.69, 3052.73, 3050.09, 3045.44, 2984.43, 2980.66, 2980.48, 2884.63, 1493.67, 1489.97, 1478.87, 1471.11, 1466.13, 1404.78, 1384.21, 1333.75, 1287.39, 1194.11, 1158.52, 1043.24, 919.47, 897.22, $858.64,750.43,633.21,607.67,367.15,341.39,273.76,221.14,200.61,130.81,72.62,58.84,49.43,24.53,6.38 \mathrm{~cm}^{\wedge}-1$

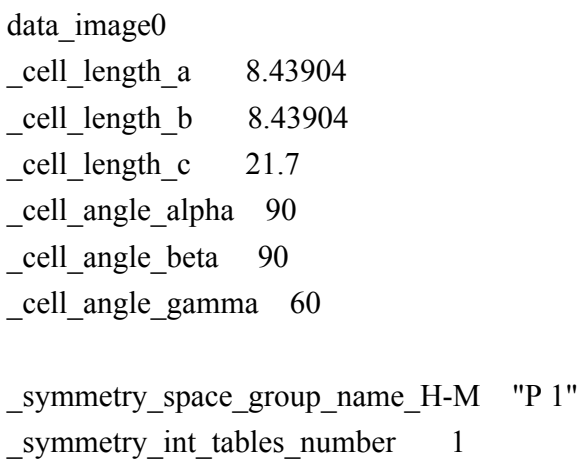




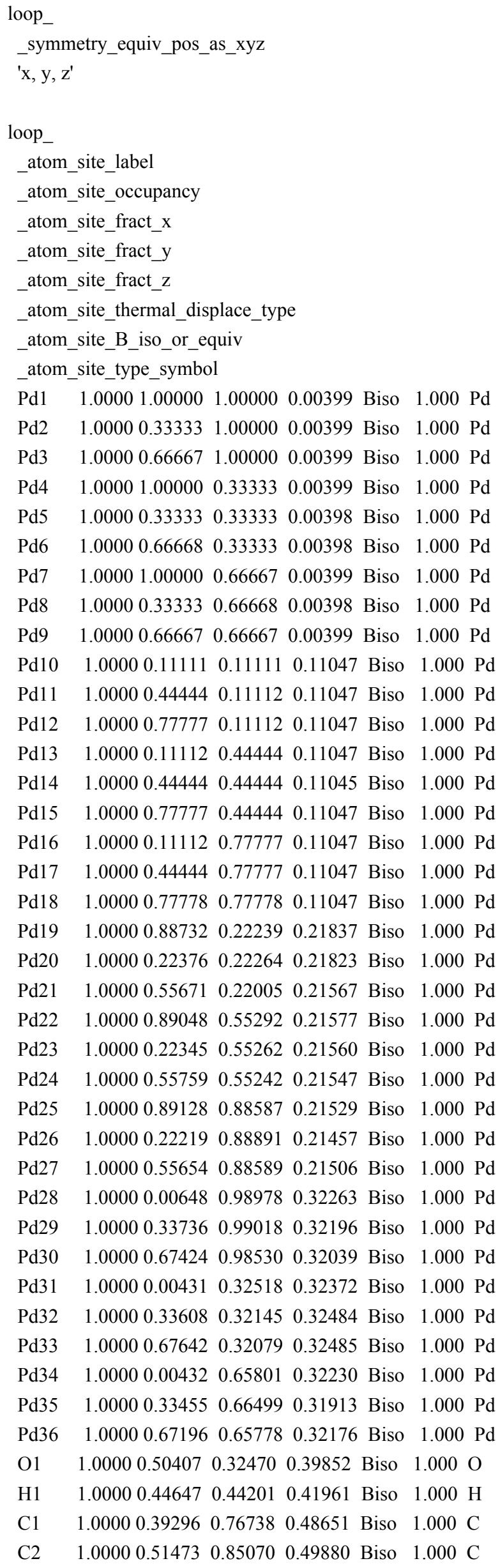


C3 $\quad \begin{array}{lllllll}1.0000 & 0.19220 & 0.89440 & 0.50367 & \text { Biso } & 1.000 \mathrm{C}\end{array}$

H2 $1.00000 .44658 \quad 0.637840 .51174$ Biso $1.000 \mathrm{H}$

H3 $1.00000 .40130 \quad 0.733430 .43713$ Biso $1.000 \mathrm{H}$

H4 1.00000 .656880 .756550 .48575 Biso $1.000 \mathrm{H}$

H5 1.00000 .108630 .831810 .49351 Biso $1.000 \mathrm{H}$

H6 $1.0000 \quad 0.46788 \quad 0.977370 .47261$ Biso $1.000 \mathrm{H}$

H7 $1.0000 \quad 0.512840 .88345 \quad 0.54768$ Biso $1.000 \mathrm{H}$

H8 $1.0000 \quad 0.17806 \quad 0.927640 .55283$ Biso $1.000 \mathrm{H}$

H9 1.00000 .134700 .023140 .47799 Biso $1.000 \mathrm{H}$

\# Pd1.C1.T5; $\mathrm{E}=-121.79 ; \mathrm{ZPVE}=2.97 \mathrm{eV}$; frequencies= 1597.36i, 3716.65, 3054.92, 3052.11, 3017.96, 2984.68, 2981.52, 2943.65, 2863.21, 1494.48, 1484.73, 1476.88, 1463.56, 1387.61, 1342.16, 1312.35, 1283.13, 1243.09, 1184.60, 1117.70, 1029.92, 992.35, 938.23, $870.56,763.27,718.90,589.81,541.31,447.61,370.07,318.66,254.24,191.67,162.03,103.98,87.09,52.51,37.33,26.73 \mathrm{~cm}^{\wedge}-1$

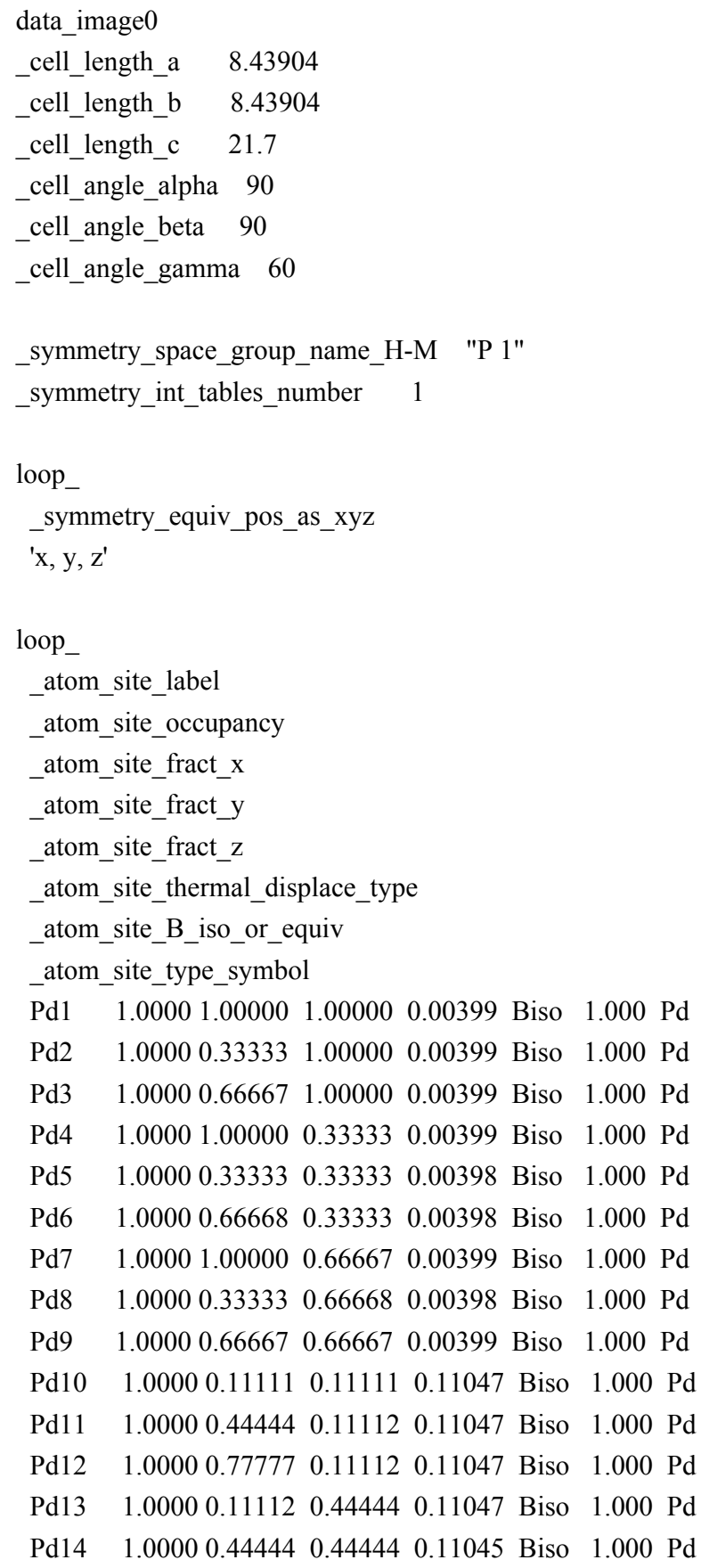




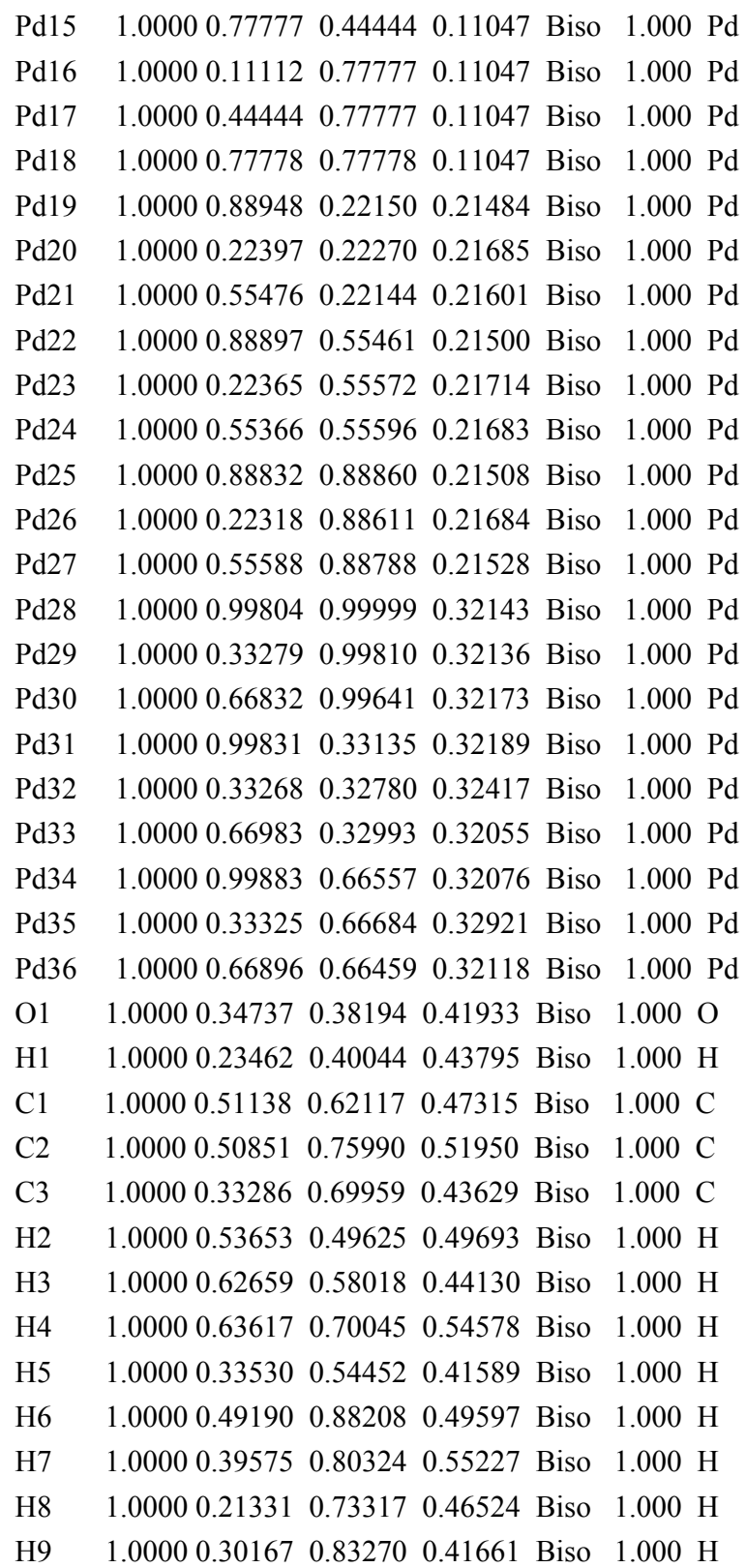

\# Pd1.C1.1p; $\mathrm{E}=-123.17$; ZPVE= 3.07 eV; frequencies=24.70i, 3833.50, 3680.65, 3075.27, 3069.58, 3063.54, 3003.35, 2991.40, 2986.28, 2964.84, 1607.48, 1487.76, 1475.48, 1461.90, 1436.82, 1380.49, 1292.23, 1282.75, 1204.62, 1112.22, 1025.00, 971.84, 939.31, 886.86, $775.94,641.45,527.77,241.52,232.31,223.92,162.68,136.77,108.92,90.06,52.28,44.75,28.67,25.44,14.18 \mathrm{~cm}^{\wedge}-1$

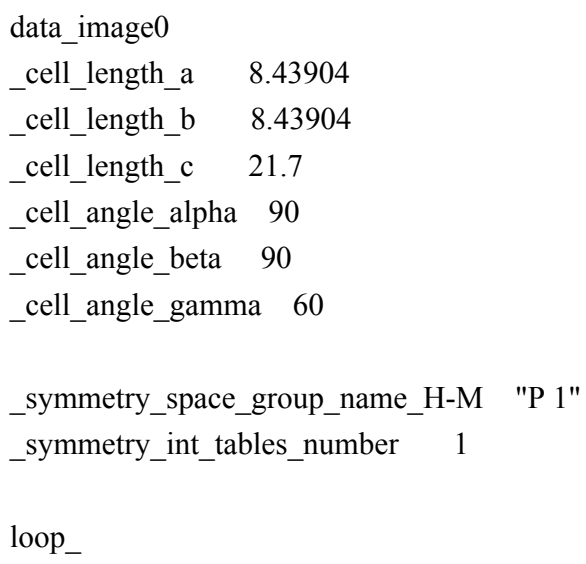




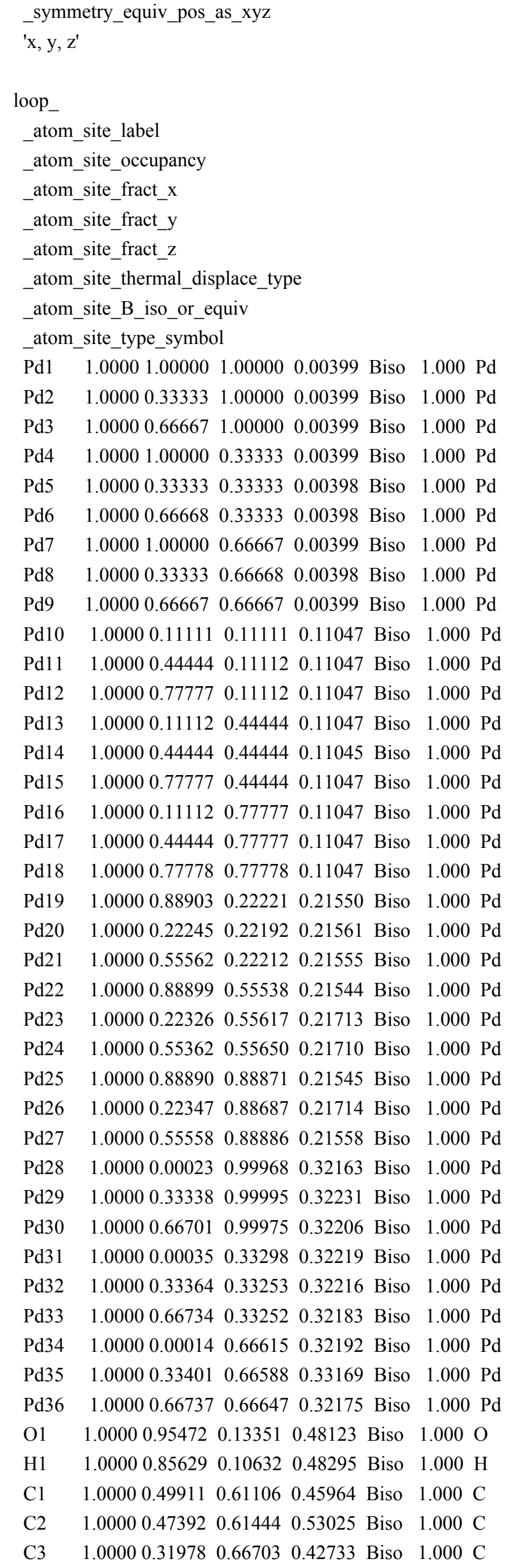




$\begin{array}{llllllll}\text { H2 } & 1.0000 & 0.60420 & 0.47309 & 0.44580 & \text { Biso } & 1.000 \mathrm{H} \\ \text { H3 } & 1.0000 & 0.54995 & 0.70392 & 0.44751 & \text { Biso } & 1.000 \mathrm{H} \\ \text { H4 } & 1.0000 & 0.60452 & 0.57129 & 0.55324 & \text { Biso } & 1.000 \mathrm{H} \\ \text { H5 } & 1.0000 & 0.94541 & 0.18344 & 0.44023 & \text { Biso } & 1.000 \mathrm{H} \\ \text { H6 } & 1.0000 & 0.37449 & 0.75190 & 0.54607 & \text { Biso } & 1.000 \mathrm{H} \\ \text { H7 } & 1.0000 & 0.42561 & 0.52123 & 0.54411 & \text { Biso } & 1.000 \mathrm{H} \\ \text { H8 } & 1.0000 & 0.26903 & 0.57249 & 0.43631 & \text { Biso } & 1.000 \mathrm{H} \\ \text { H9 } & 1.0000 & 0.21424 & 0.80745 & 0.43778 & \text { Biso } & 1.000 \mathrm{H}\end{array}$

\# Pd2.C1.P; $E=-122.61 ; Z P V E=3.12 \mathrm{eV}$; frequencies= 23.14i, 3684.13, 3052.29, 3045.09, 3039.11, 3022.34, 2998.94, 2977.83, 2973.16, 2954.60, 1492.16, 1488.69, 1476.89, 1469.43, 1466.41, 1397.40, 1376.43, 1345.23, 1296.47, 1188.27, 1153.77, 1042.85, 918.96, 896.63, $857.74,739.79,676.22,638.19,368.15,340.31,273.38,236.68,223.38,100.09,54.66,48.25,42.93,31.10,12.93 \mathrm{~cm}^{\wedge}-1$

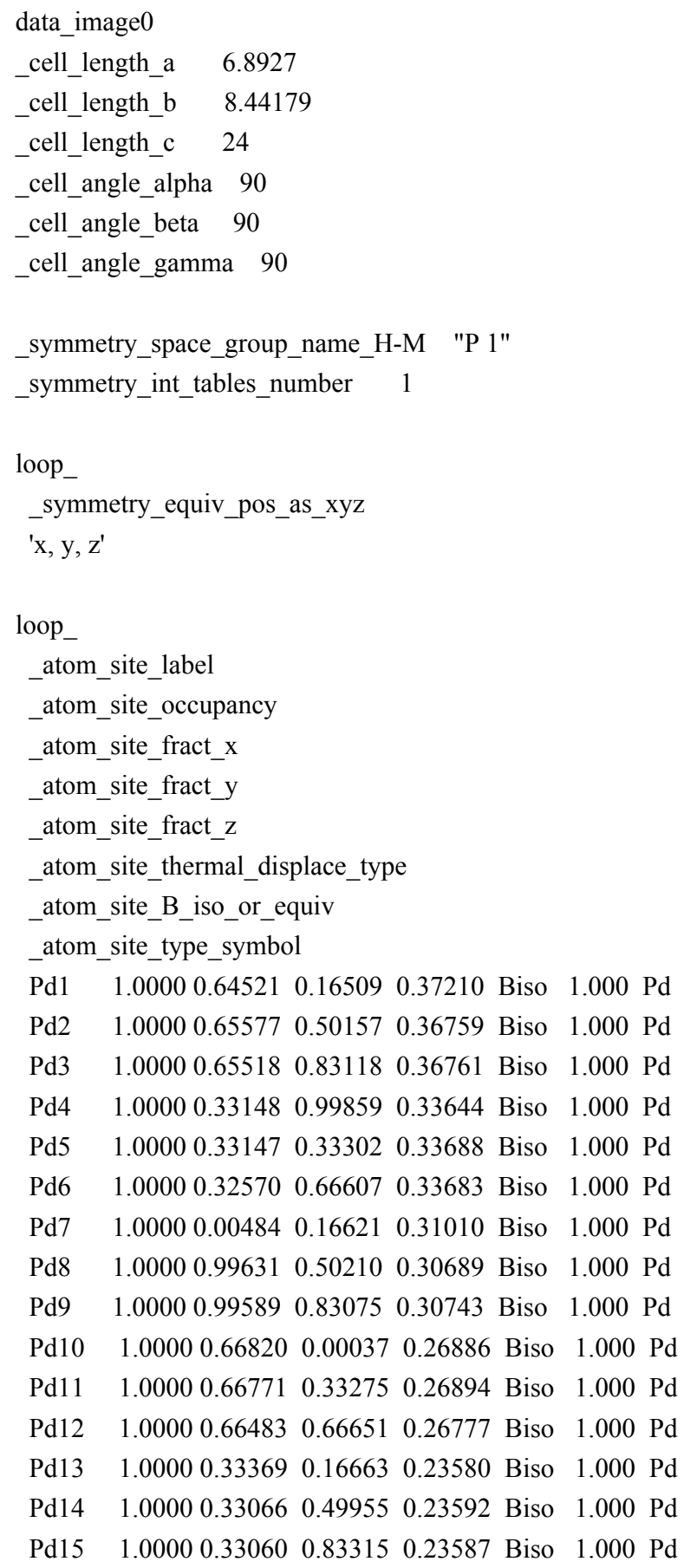




\begin{tabular}{|c|c|c|c|}
\hline Pd16 & 1.00000 .000620 .00141 & 0.20460 Biso & 1.000 \\
\hline Pd17 & 1.00000 .000330 .33162 & 20.20461 Biso & 1.00 \\
\hline Pd18 & 1.00000 .999990 .66667 & 70.20322 Biso & 1.000 \\
\hline Pd19 & $\begin{array}{lll}1.0000 & 0.66897 & 0.16675\end{array}$ & 50.16784 Biso & 1.000 \\
\hline $\operatorname{Pd} 20$ & $\begin{array}{lll}1.0000 & 0.66897 & 0.50008\end{array}$ & 0.16784 Biso & 1.000 \\
\hline $\operatorname{Pd} 21$ & $\begin{array}{lll}1.0000 & 0.66897 & 0.83342\end{array}$ & 20.16784 Biso & 1.000 \\
\hline $\operatorname{Pd} 22$ & $\begin{array}{lll}1.0000 & 0.33838 & 0.00008\end{array}$ & 0.13524 Biso & 1.000 \\
\hline $\operatorname{Pd} 23$ & $\begin{array}{lll}1.0000 & 0.33838 & 0.33342\end{array}$ & 20.13524 Biso & 1.000 \\
\hline $\operatorname{Pd} 24$ & 1.00000 .338380 .66675 & 50.13524 Biso & 1.000 \\
\hline $\operatorname{Pd} 25$ & 1.00000 .002020 .16675 & 50.10321 Biso & 1.000 \\
\hline $\operatorname{Pd} 26$ & 1.00000 .002020 .50008 & 0.10321 Biso & 1.000 \\
\hline $\operatorname{Pd} 27$ & 1.00000 .002020 .83342 & 0.10321 Biso & 1.000 \\
\hline $\operatorname{Pd} 28$ & $\begin{array}{lll}1.0000 & 0.66998 & 0.00008\end{array}$ & 80.06364 Biso & 1.000 \\
\hline $\operatorname{Pd} 29$ & $\begin{array}{lll}1.0000 & 0.66998 & 0.33342\end{array}$ & 20.06364 Biso & 1.000 \\
\hline $\operatorname{Pd} 30$ & 1.00000 .669980 .66675 & 50.06364 Biso & 1.000 \\
\hline $\operatorname{Pd} 31$ & 1.00000 .339310 .16675 & 0.03394 Biso & 1.000 \\
\hline $\operatorname{Pd} 32$ & $\begin{array}{lll}1.0000 & 0.33931 & 0.50008\end{array}$ & 0.03394 Biso & 1.000 \\
\hline $\operatorname{Pd} 33$ & $\begin{array}{lll}1.0000 & 0.33931 & 0.83342\end{array}$ & 20.03394 Biso & 1.000 \\
\hline $\operatorname{Pd} 34$ & $\begin{array}{lll}1.0000 & 0.00766 & 0.00008\end{array}$ & 80.00384 Biso & 1.000 \\
\hline Pd35 & $\begin{array}{lll}1.0000 & 0.00766 & 0.33342\end{array}$ & 20.00384 Biso & 1.000 \\
\hline $\operatorname{Pd} 36$ & $1.00000 .00766 \quad 0.66675$ & 0.00384 Biso & 1.000 \\
\hline $\mathrm{O} 1$ & 1.00000 .936630 .17240 & 0.39817 Biso & 1.000 \\
\hline $\mathrm{H} 1$ & 1.00000 .972680 .06870 & 0.41251 Biso & 1.000 \\
\hline $\mathrm{C} 1$ & $\begin{array}{lll}1.0000 & 0.09082 & 0.50137\end{array}$ & 0.48993 Biso & 1.000 \\
\hline $\mathrm{C} 2$ & 1.00000 .227370 .64117 & 0.50056 Biso & 1.000 \\
\hline $\mathrm{C} 3$ & 1.00000 .877930 .54109 & 0.50159 Biso & 1.000 \\
\hline $\mathrm{H} 2$ & 1.00000 .134550 .40059 & 0.51592 Biso & 1.000 \\
\hline H3 & $1.0000 \quad 0.105650 .46156$ & 0.44667 Biso & 1.000 \\
\hline $\mathrm{H} 4$ & 1.00000 .378530 .60926 & 0.49225 Biso & 1.000 \\
\hline H5 & $\begin{array}{lll}1.0000 & 0.78383 & 0.43919\end{array}$ & 0.49349 Biso & 1.000 \\
\hline H6 & $1.0000 \quad 0.190830 .74188$ & 0.47369 Biso & 1.000 \\
\hline H7 & $1.0000 \quad 0.21806 \quad 0.68154$ & 0.54384 Biso & 1.000 \\
\hline H8 & $\begin{array}{lll}1.0000 & 0.85611 & 0.57697\end{array}$ & 0.54500 Biso & 1.000 \\
\hline H9 & 1.00000 .828750 .63959 & 0.47525 Biso & 1.000 \\
\hline
\end{tabular}

\# Pd2.C1.T5; $E=-121.58 ; \mathrm{ZPVE}=2.97 \mathrm{eV}$; frequencies= 1563.53i, 3729.21, 3055.83, 3046.31, 3018.02, 2983.62, 2978.98, 2974.85, $2773.95,1486.76,1479.75,1470.40,1466.55,1387.73,1348.80,1333.26,1293.25,1252.15,1178.58,1112.74,1033.10,996.58,925.54$, $871.88,754.60,722.46,566.19,542.50,482.87,366.82,317.87,262.27,209.50,166.75,104.76,94.07,58.26,42.08,24.54 \mathrm{~cm}^{\wedge}-1$

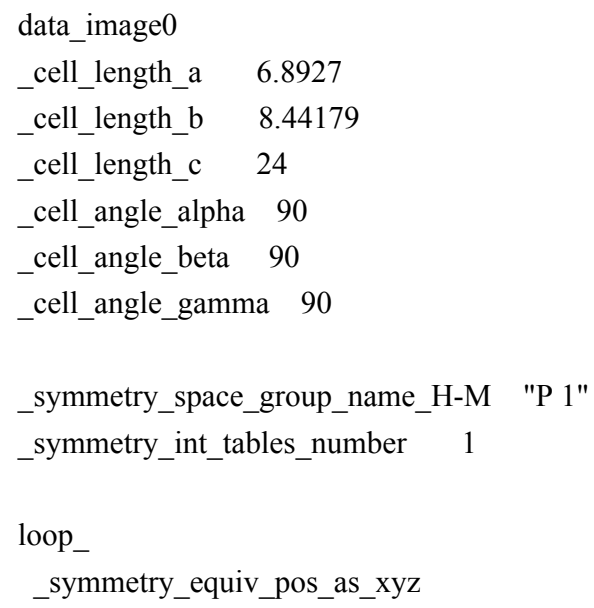




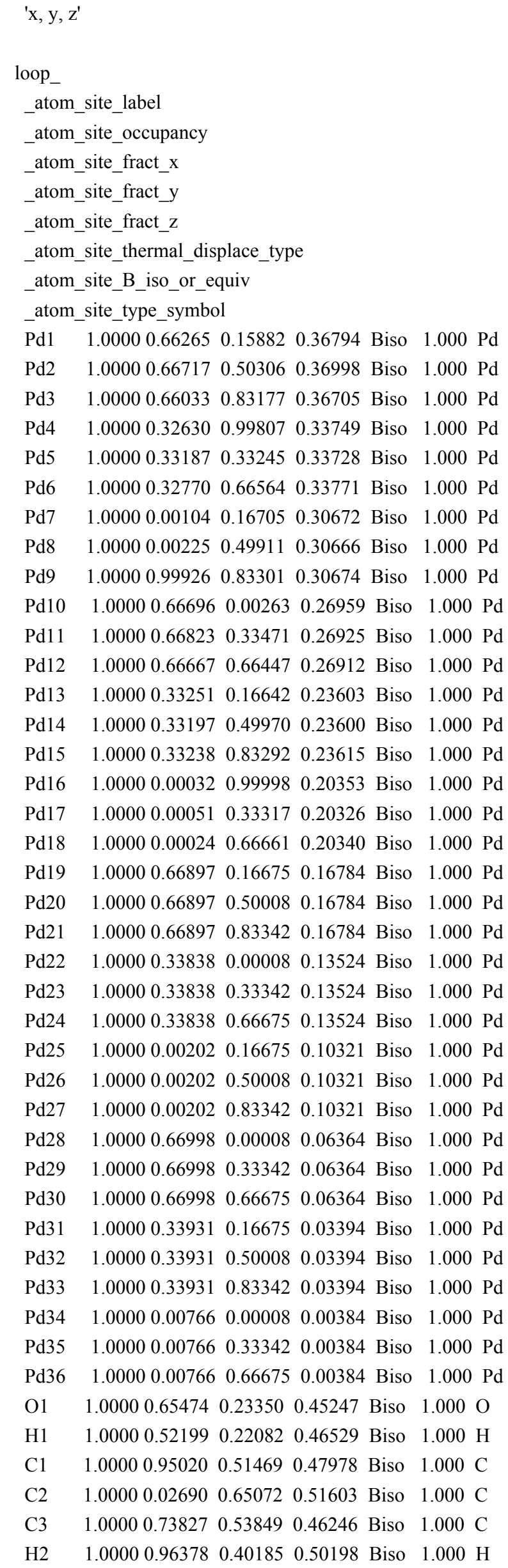




$\begin{array}{llllllll}\text { H3 } & 1.0000 & 0.04090 & 0.50466 & 0.44242 & \text { Biso } & 1.000 \mathrm{H} \\ \text { H4 } & 1.0000 & 0.17803 & 0.62973 & 0.52799 & \text { Biso } & 1.000 \mathrm{H} \\ \text { H5 } & 1.0000 & 0.68059 & 0.38737 & 0.44744 & \text { Biso } & 1.000 \mathrm{H} \\ \text { H6 } & 1.0000 & 0.02077 & 0.76343 & 0.49352 & \text { Biso } & 1.000 \mathrm{H} \\ \text { H7 } & 1.0000 & 0.94148 & 0.66345 & 0.55419 & \text { Biso } & 1.000 \mathrm{H} \\ \text { H8 } & 1.0000 & 0.63671 & 0.53609 & 0.49774 & \text { Biso } & 1.000 \mathrm{H} \\ \text { H9 } & 1.0000 & 0.71995 & 0.65974 & 0.44526 & \text { Biso } & 1.000 \mathrm{H}\end{array}$

\# Pd2.C1.1p; $E=-122.75 ; Z P V E=3.10 \mathrm{eV}$; frequencies=3797.20, 3683.33, 3066.14, 3051.21, 3038.78, 3012.74, 2984.65, 2979.01, 2955.13, $1612.70,1488.50,1476.48,1464.76,1421.82,1380.56,1308.77,1295.04,1196.95,1103.39,1015.81,977.36,928.36,879.76,762.02$, $620.26,531.65,396.84,353.66,245.67,241.54,142.28,124.10,119.08,92.95,74.89,50.80,39.59,32.44,22.90 \mathrm{~cm}^{\wedge}-1$

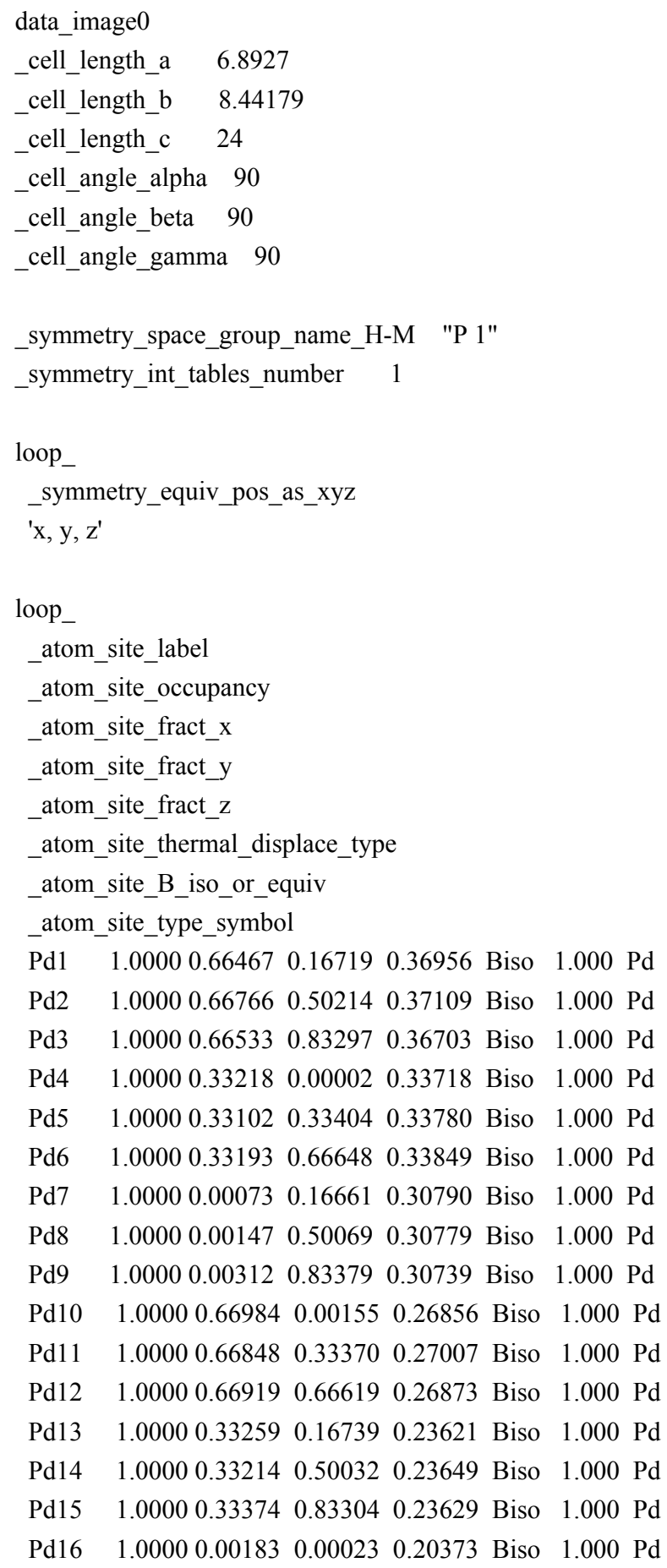




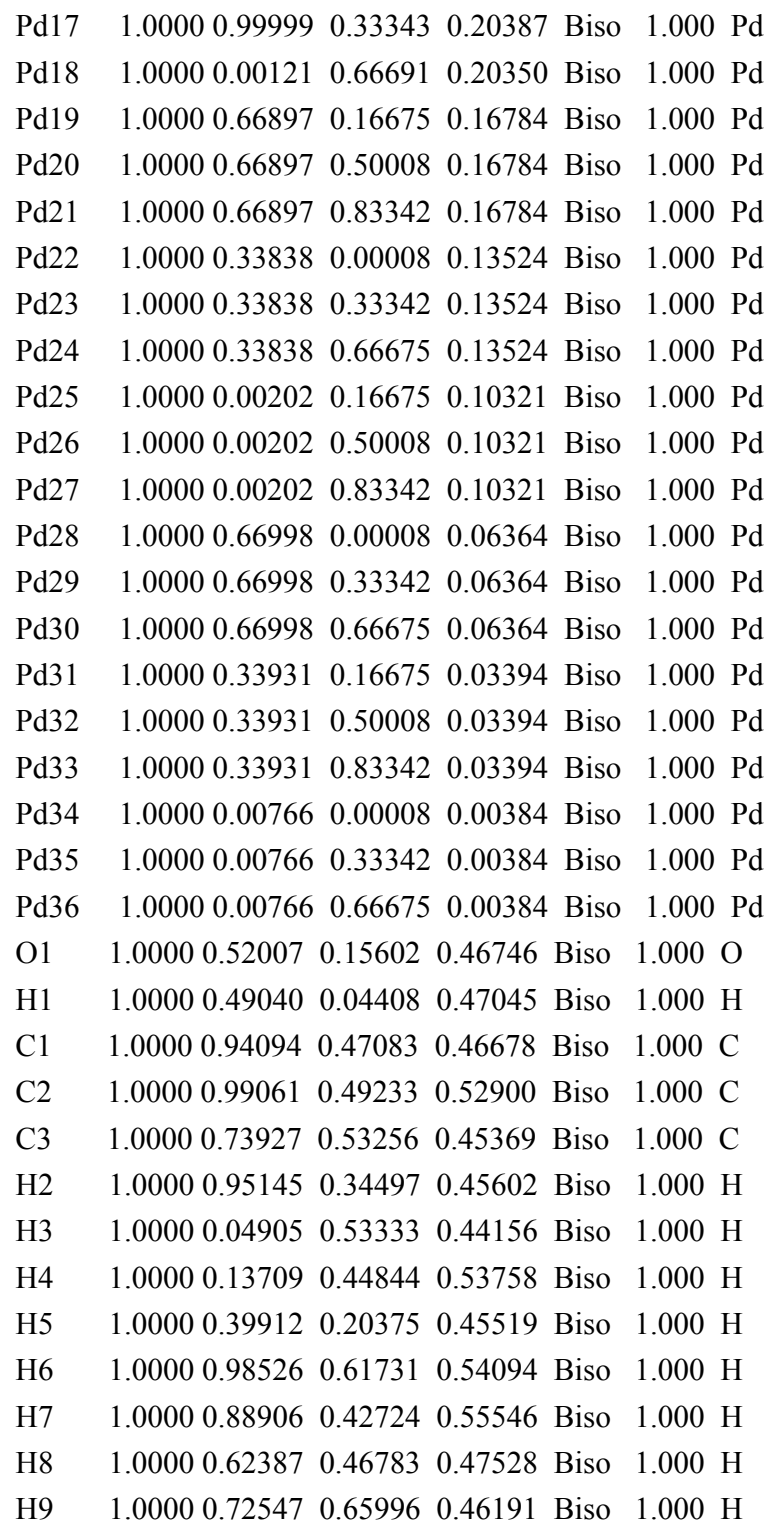

\# Pd1.C2.P; $E=-123.04 ; Z P V E=3.12 \mathrm{eV}$; frequencies=11.38i, 29.91i, 3683.57, 3056.25, 3052.84, 3044.52, 3026.96, 2984.42, 2983.43, 2968.20, 2958.36, 1493.28, 1488.48, 1478.47, 1469.11, 1465.54, 1401.24, 1380.20, 1342.02, 1298.12, 1193.98, 1156.06, 1045.83, 919.95, $900.94,859.05,749.58,627.96,603.67,367.47,335.33,266.46,214.54,196.57,131.03,57.12,40.47,33.35,15.24 \mathrm{~cm}^{\wedge}-1$

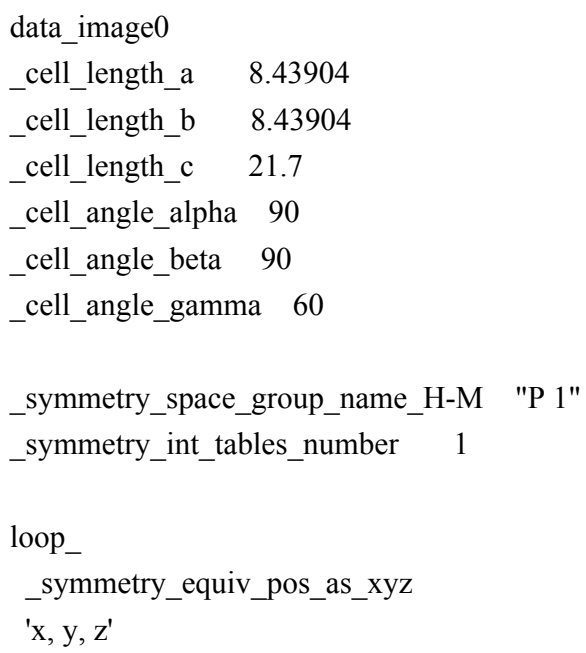




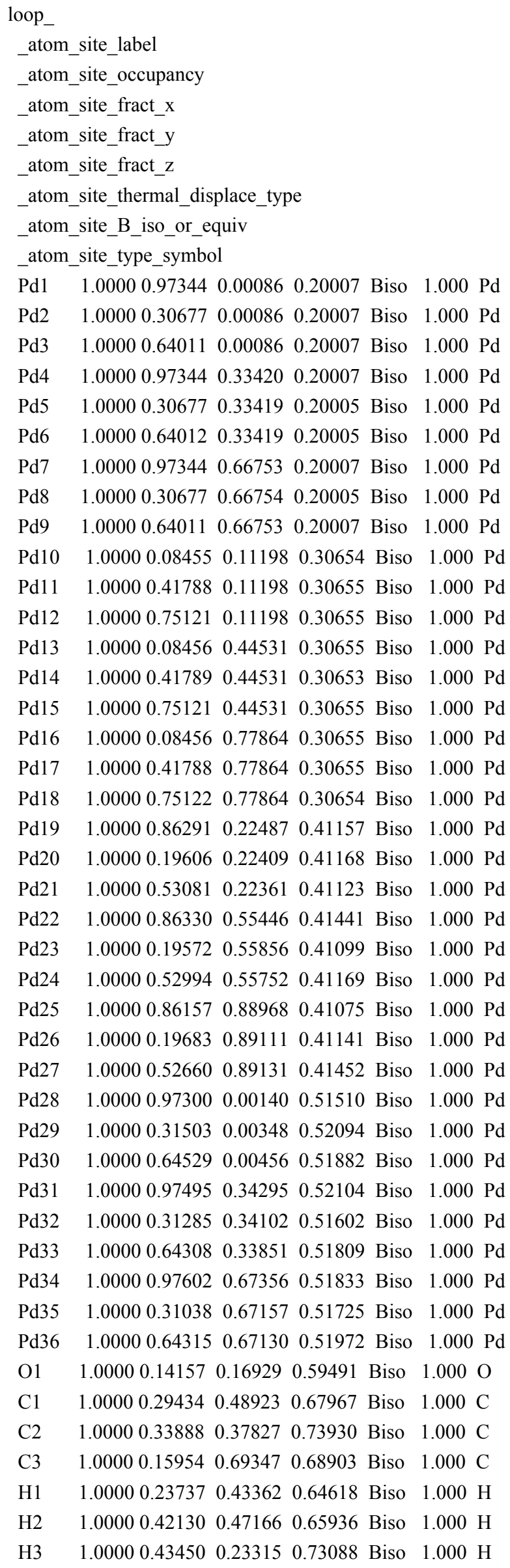




$\begin{array}{llllllll}\text { H4 } & 1.0000 & 0.12951 & 0.76807 & 0.64531 & \text { Biso } & 1.000 \mathrm{H} \\ \text { H5 } & 1.0000 & 0.40065 & 0.42665 & 0.77339 & \text { Biso } & 1.000 \mathrm{H} \\ \text { H6 } & 1.0000 & 0.21493 & 0.38987 & 0.75975 & \text { Biso } & 1.000 \mathrm{H} \\ \text { H7 } & 1.0000 & 0.02958 & 0.71585 & 0.70791 & \text { Biso } & 1.000 \mathrm{H} \\ \text { H8 } & 1.0000 & 0.21469 & 0.75446 & 0.72089 & \text { Biso } & 1.000 \mathrm{H} \\ \text { H9 } & 1.0000 & 0.08307 & 0.10934 & 0.61534 & \text { Biso } & 1.000 \mathrm{H}\end{array}$

\# Pd1.C2.T5; E= -121.67; ZPVE= 2.97 eV; frequencies= 74.07i, 237.12i, 3789.74, 3067.21, 3051.96, 3031.99, 3008.97, 2997.32, 2897.98, 2890.69, 1957.16, 1512.46, 1469.29, 1457.27, 1452.38, 1446.60, 1382.43, 1372.62, 1349.52, 1166.86, 1118.75, 1010.25, 928.18, 915.53, $860.98,826.45,700.00,599.36,375.48,303.70,219.90,190.30,177.15,105.24,90.58,63.92,42.62,27.07,19.88 \mathrm{~cm}^{\wedge}-1$

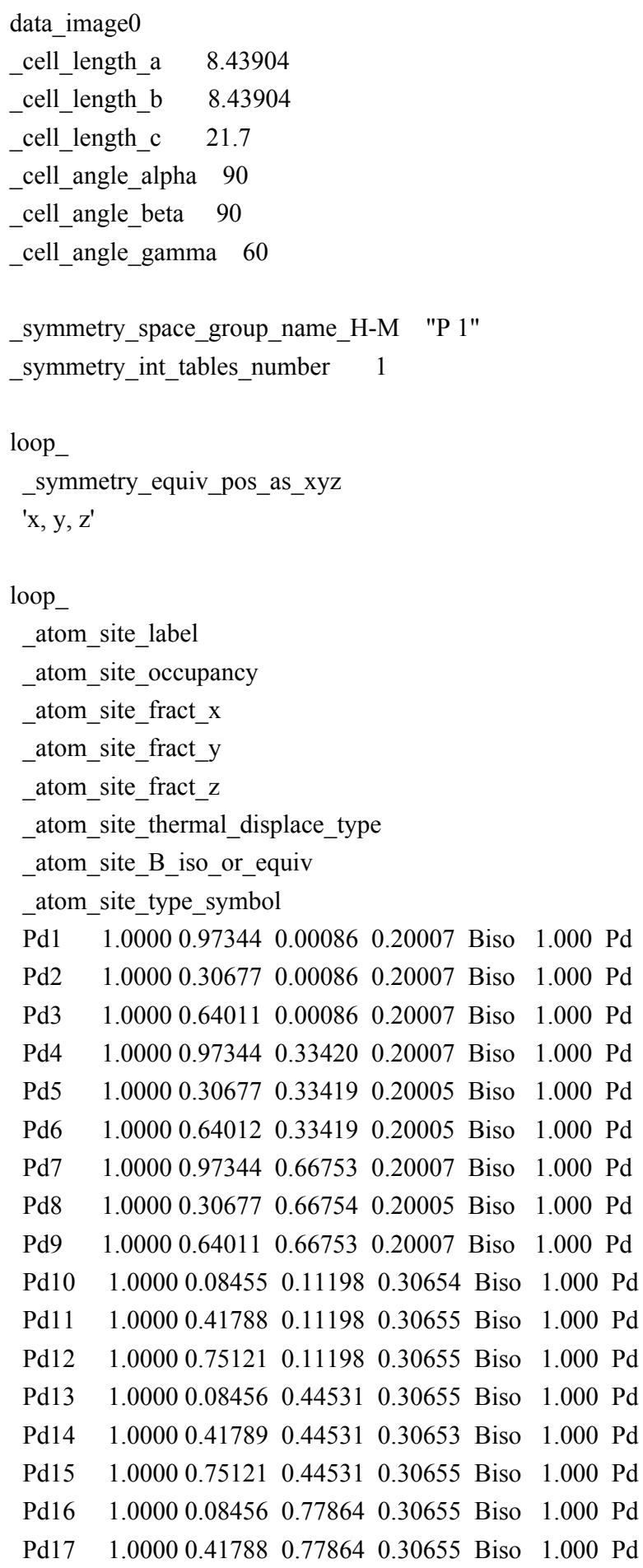




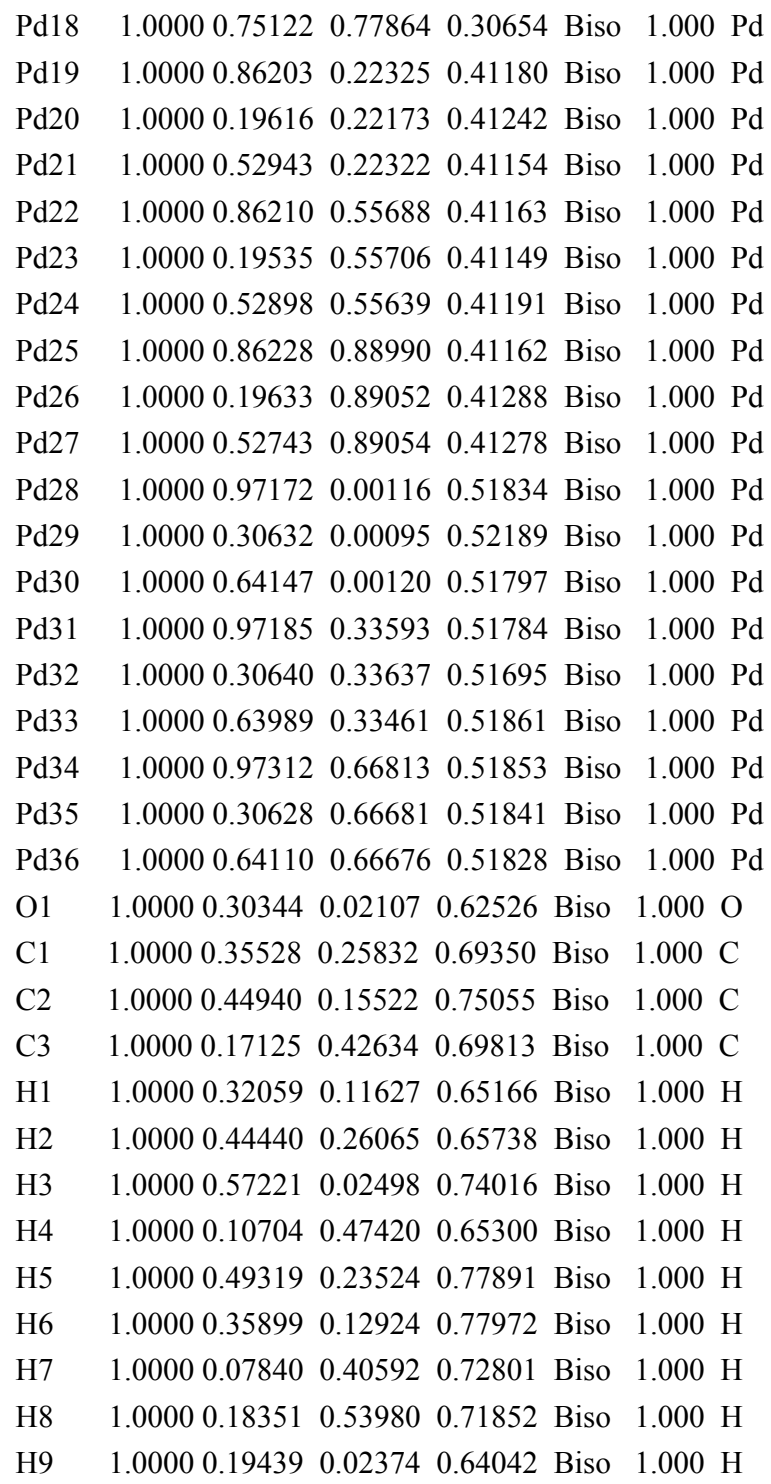

\# Pd1.C2.2p; E= -123.23; ZPVE=3.06 eV; frequencies=20.25i, 3831.39, 3646.44, 3053.41, 3049.52, 3014.98, 3008.10, 3007.15, 2939.39, 2931.80, 1604.60, 1471.98, 1453.02, 1450.44, 1441.93, 1378.57, 1364.41, 1328.56, 1172.61, 1087.70, 1086.52, 914.97, 903.69, 891.00, $853.49,424.66,384.25,241.60,235.89,213.19,199.83,194.25,137.86,104.08,73.81,64.90,55.92,39.06,32.62 \mathrm{~cm}^{\wedge}-1$

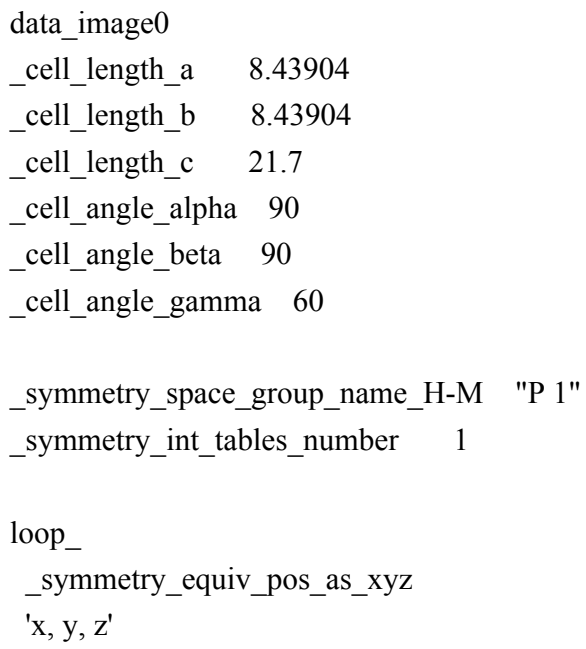




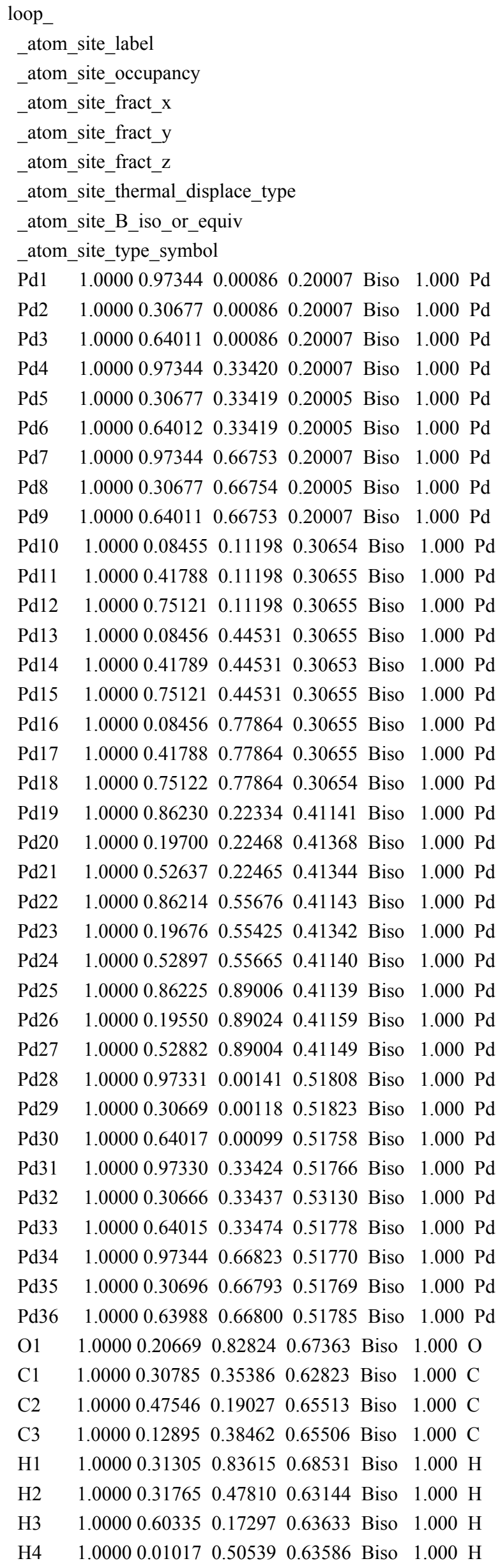




$\begin{array}{llllllll}\text { H5 } & 1.0000 & 0.47767 & 0.21200 & 0.70514 & \text { Biso } & 1.000 \mathrm{H} \\ \text { H6 } & 1.0000 & 0.47344 & 0.06263 & 0.64884 & \text { Biso } & 1.000 \mathrm{H} \\ \text { H7 } & 1.0000 & 0.11053 & 0.26594 & 0.64948 & \text { Biso } & 1.000 \mathrm{H} \\ \text { H8 } & 1.0000 & 0.12985 & 0.40891 & 0.70493 & \text { Biso } & 1.000 \mathrm{H} \\ \text { H9 } & 1.0000 & 0.18482 & 0.86811 & 0.63089 & \text { Biso } & 1.000 \mathrm{H}\end{array}$

\# Pd2.C2.P E= -122.56; ZPVE= 3.11 eV; frequencies= 11.46i, 3749.85, 3059.25, 3052.88, 3046.36, 3036.26, 2987.70, 2982.97, 2975.28, 2902.50, 1491.48, 1486.02, 1477.35, 1468.63, 1459.75, 1406.44, 1385.93, 1339.75, 1292.70, 1191.70, 1153.61, 1045.16, 917.52, 899.82, $855.74,749.58,491.96,433.76,368.91,321.29,260.56,209.20,179.76,168.47,75.07,58.81,52.66,43.64,19.02 \mathrm{~cm}^{\wedge}-1$

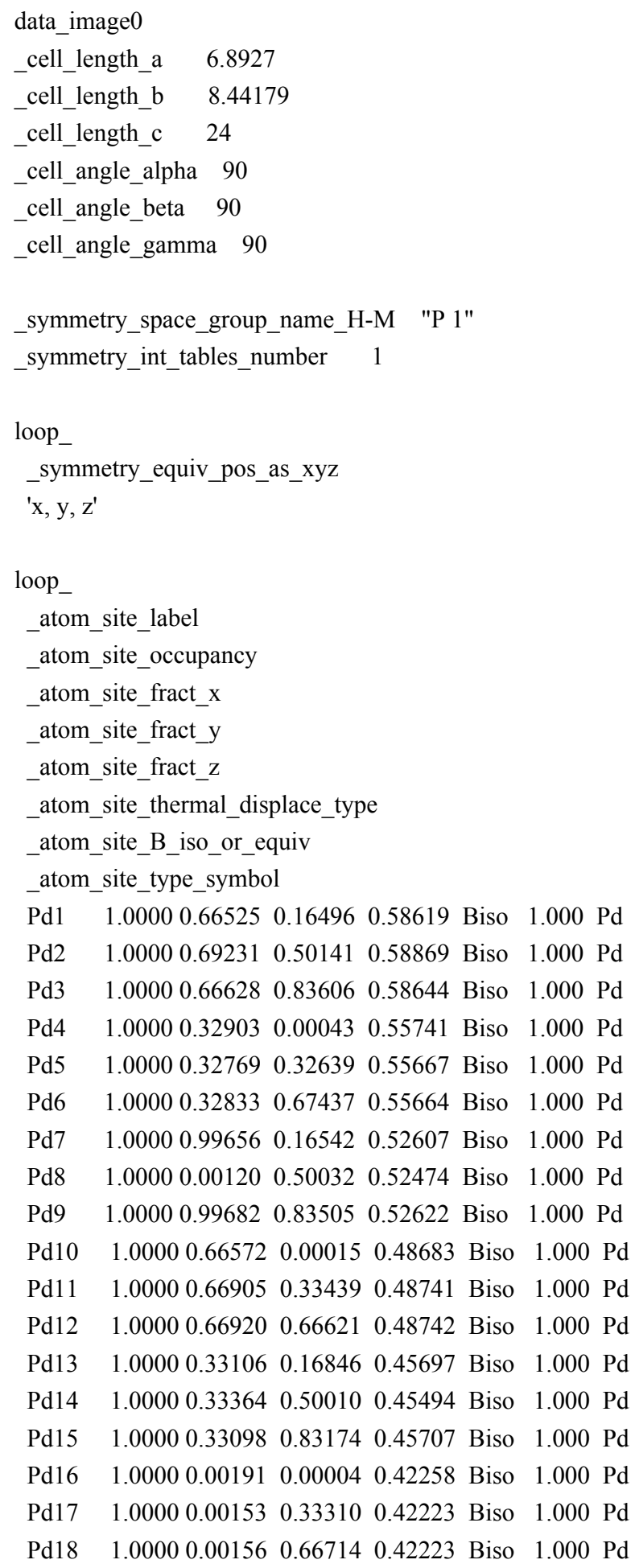




$\begin{array}{llllllll}\mathrm{Pd} 19 & 1.0000 & 0.66897 & 0.16673 & 0.38692 & \text { Biso } & 1.000 \mathrm{Pd} \\ \mathrm{Pd} 20 & 1.0000 & 0.66897 & 0.50006 & 0.38692 & \text { Biso } & 1.000 \mathrm{Pd} \\ \mathrm{Pd} 21 & 1.0000 & 0.66897 & 0.83340 & 0.38692 & \text { Biso } & 1.000 \mathrm{Pd} \\ \mathrm{Pd} 22 & 1.0000 & 0.33838 & 0.00006 & 0.35432 & \text { Biso } & 1.000 \mathrm{Pd} \\ \mathrm{Pd} 23 & 1.0000 & 0.33838 & 0.33340 & 0.35432 & \text { Biso } & 1.000 \mathrm{Pd} \\ \mathrm{Pd} 24 & 1.0000 & 0.33838 & 0.66673 & 0.35432 & \text { Biso } & 1.000 \mathrm{Pd} \\ \mathrm{Pd} 25 & 1.0000 & 0.00202 & 0.16673 & 0.32230 & \text { Biso } & 1.000 \mathrm{Pd} \\ \mathrm{Pd} 26 & 1.0000 & 0.00202 & 0.50006 & 0.32230 & \text { Biso } & 1.000 \mathrm{Pd} \\ \mathrm{Pd} 27 & 1.0000 & 0.00202 & 0.83340 & 0.32230 & \text { Biso } & 1.000 \mathrm{Pd} \\ \mathrm{Pd} 28 & 1.0000 & 0.66998 & 0.00006 & 0.28273 & \text { Biso } & 1.000 \mathrm{Pd} \\ \mathrm{Pd} 29 & 1.0000 & 0.66998 & 0.33340 & 0.28273 & \text { Biso } & 1.000 \mathrm{Pd} \\ \mathrm{Pd} 30 & 1.0000 & 0.66998 & 0.66673 & 0.28273 & \text { Biso } & 1.000 \mathrm{Pd} \\ \mathrm{Pd} 31 & 1.0000 & 0.33931 & 0.16673 & 0.25303 & \text { Biso } & 1.000 \mathrm{Pd} \\ \mathrm{Pd} 32 & 1.0000 & 0.33931 & 0.50006 & 0.25303 & \text { Biso } & 1.000 \mathrm{Pd} \\ \mathrm{Pd} 33 & 1.0000 & 0.33931 & 0.83340 & 0.25303 & \text { Biso } & 1.000 \mathrm{Pd} \\ \mathrm{Pd} 34 & 1.0000 & 0.00766 & 0.00006 & 0.22292 & \text { Biso } & 1.000 \mathrm{Pd} \\ \mathrm{Pd} 35 & 1.0000 & 0.00766 & 0.33340 & 0.22292 & \text { Biso } & 1.000 \mathrm{Pd} \\ \mathrm{Pd} 36 & 1.0000 & 0.00766 & 0.66673 & 0.22292 & \text { Biso } & 1.000 \mathrm{Pd} \\ \mathrm{O} 1 & 1.0000 & 0.39566 & 0.50199 & 0.62216 & \text { Biso } & 1.000 \mathrm{O} \\ \mathrm{C} 1 & 1.0000 & 0.93759 & 0.40986 & 0.71462 & \text { Biso } & 1.000 \mathrm{C} \\ \mathrm{C} 2 & 1.0000 & 0.03895 & 0.53678 & 0.74966 & \text { Biso } & 1.000 \mathrm{C} \\ \mathrm{C} 3 & 1.0000 & 0.03744 & 0.24798 & 0.71668 & \text { Biso } & 1.000 \mathrm{C} \\ \mathrm{H} 1 & 1.0000 & 0.92935 & 0.45002 & 0.67101 & \text { Biso } & 1.000 \mathrm{H} \\ \mathrm{H} 2 & 1.0000 & 0.78707 & 0.39768 & 0.72888 & \text { Biso } & 1.000 \mathrm{H} \\ \mathrm{H} 3 & 1.0000 & 0.96556 & 0.65121 & 0.74627 & \text { Biso } & 1.000 \mathrm{H} \\ \mathrm{H} 4 & 1.0000 & 0.96052 & 0.16098 & 0.69109 & \text { Biso } & 1.000 \mathrm{H} \\ \mathrm{H} 5 & 1.0000 & 0.04235 & 0.50341 & 0.79371 & \text { Biso } & 1.000 \mathrm{H} \\ \mathrm{H} 6 & 1.0000 & 0.18983 & 0.55428 & 0.73655 & \text { Biso } & 1.000 \mathrm{H} \\ \mathrm{H} 7 & 1.0000 & 0.18641 & 0.25456 & 0.70095 & \text { Biso } & 1.000 \mathrm{H} \\ & 1.0000 & 0.04337 & 0.20268 & 0.75937 & \text { Biso } & 1.000 \mathrm{H} \\ \mathrm{H} 9000 & 0.34702 & 0.50791 & 0.65993 & \text { Biso } & 1.000 \mathrm{H}\end{array}$

\# Pd2.C2.T5 E= -121.40; ZPVE=2.93 eV; frequencies=1084.48i, 3723.15, 3050.74, 3045.84, 2999.20, 2995.27, 2952.10, 2926.86, 2716.23, 1534.69, 1475.72, 1463.13, 1454.03, 1442.62, 1388.40, 1374.82, 1291.30, 1192.42, 1146.02, 1081.45, 1071.45, 945.81, 913.69, 891.49, $812.57,744.27,532.12,381.92,347.13,312.77,240.91,212.40,184.00,133.36,96.28,71.96,59.50,55.34,37.74 \mathrm{~cm}^{\wedge}-1$

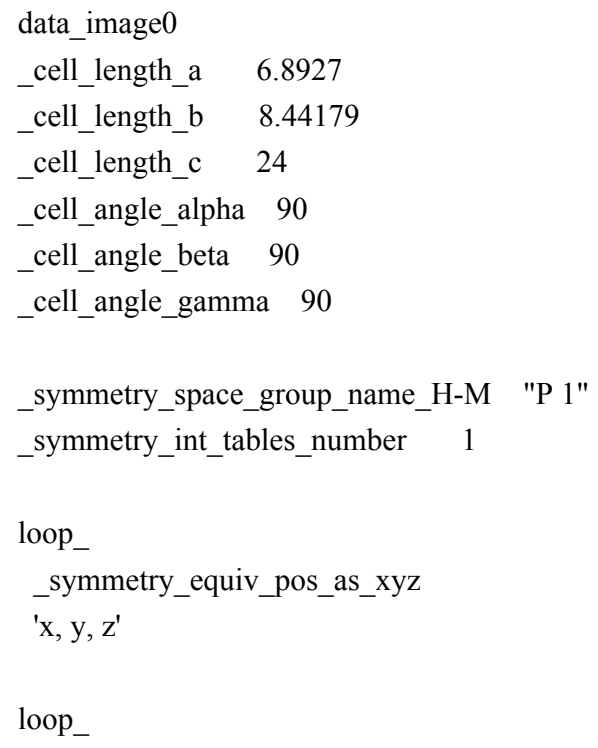




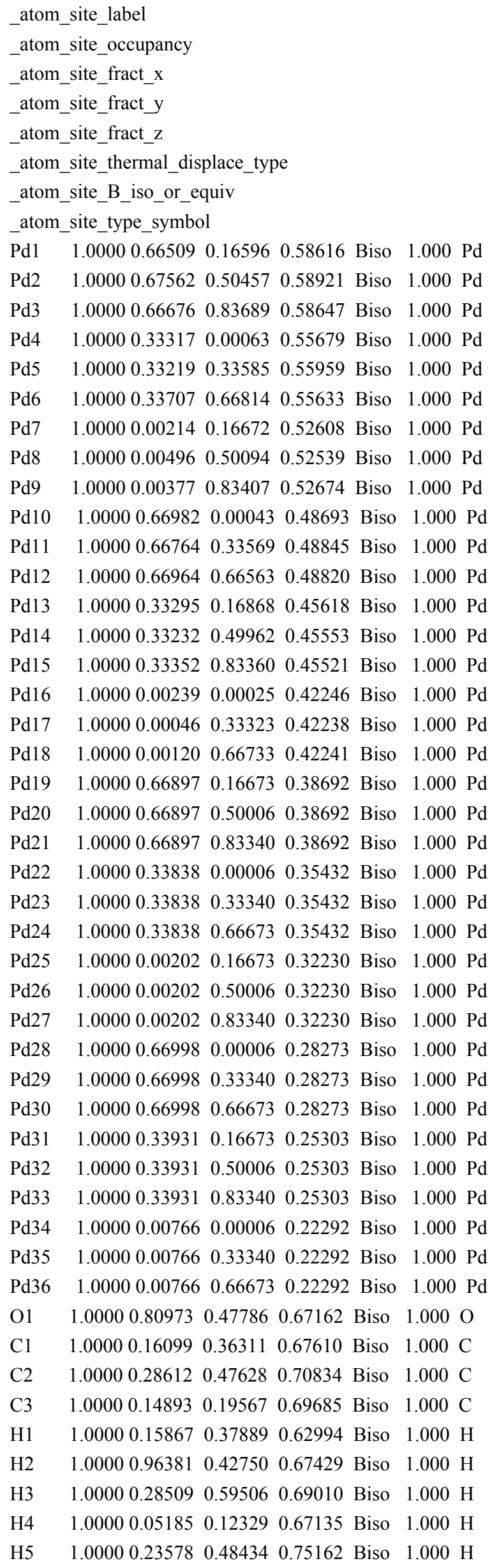


H6 $\quad 1.00000 .437880 .43661 \quad 0.71019$ Biso $1.000 \mathrm{H}$

H7 1.00000 .292190 .137800 .69690 Biso $1.000 \mathrm{H}$

H8 1.00000 .097290 .193470 .74007 Biso $1.000 \mathrm{H}$

H9 $\quad 1.00000 .817660 .580910 .68951$ Biso $1.000 \mathrm{H}$

\# Pd2.C2.2p E = -122.67; ZPVE= 3.09 eV; frequencies $=3820.29,3696.15,3045.11,3026.17,3008.85,2996.46,2983.19,2919.05,2905.46$, $1617.52,1467.23,1456.86,1445.74,1440.68,1376.94,1361.53,1316.74,1168.83,1094.31,1086.78,920.15,909.98,895.45,855.53$, $431.73,385.88,379.08,330.12,255.52,235.99,208.13,204.32,132.36,124.65,104.40,87.91,62.90,59.88,37.22 \mathrm{~cm}^{\wedge}-1$

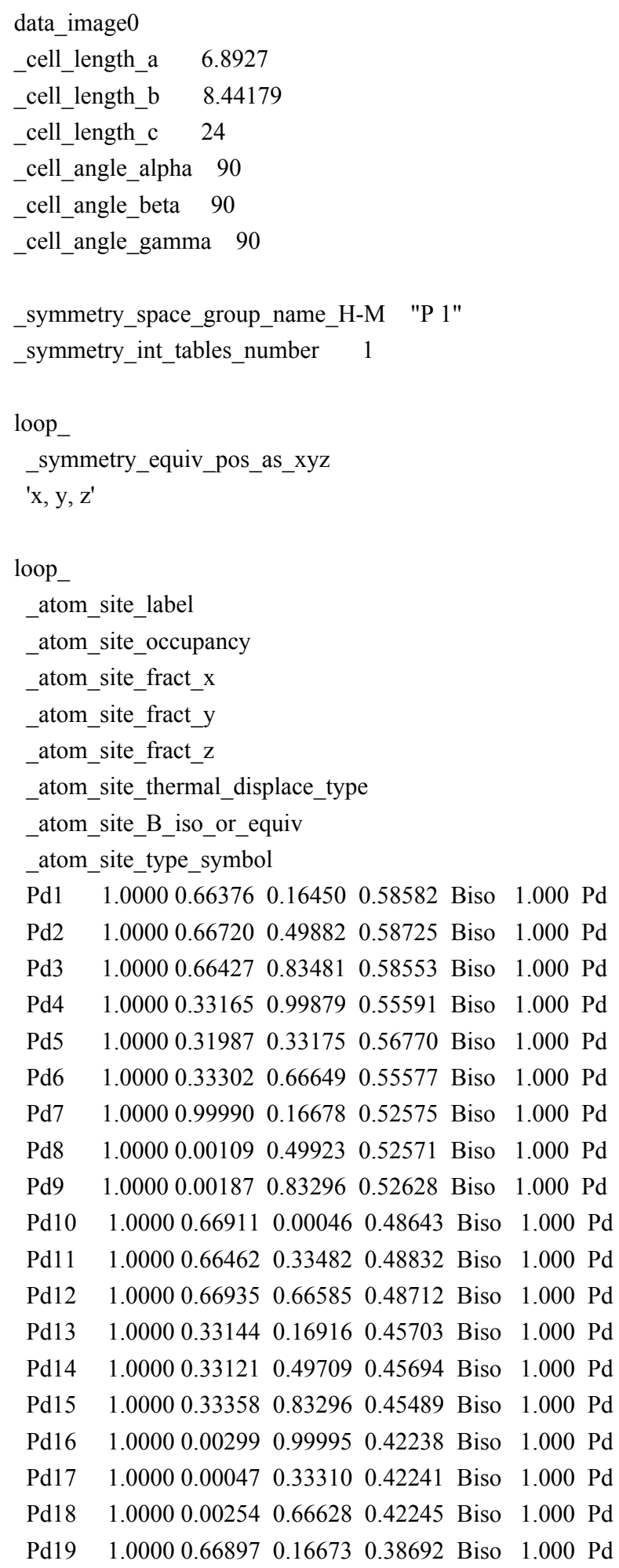




$\begin{array}{llllllll}\mathrm{Pd} 20 & 1.0000 & 0.66897 & 0.50006 & 0.38692 & \text { Biso } & 1.000 \mathrm{Pd} \\ \mathrm{Pd} 21 & 1.0000 & 0.66897 & 0.83340 & 0.38692 & \text { Biso } & 1.000 \mathrm{Pd} \\ \mathrm{Pd} 22 & 1.0000 & 0.33838 & 0.00006 & 0.35432 & \text { Biso } & 1.000 \mathrm{Pd} \\ \mathrm{Pd} 23 & 1.0000 & 0.33838 & 0.33340 & 0.35432 & \text { Biso } & 1.000 \mathrm{Pd} \\ \mathrm{Pd} 24 & 1.0000 & 0.33838 & 0.66673 & 0.35432 & \text { Biso } & 1.000 \mathrm{Pd} \\ \mathrm{Pd} 25 & 1.0000 & 0.00202 & 0.16673 & 0.32230 & \text { Biso } & 1.000 \mathrm{Pd} \\ \mathrm{Pd} 26 & 1.0000 & 0.00202 & 0.50006 & 0.32230 & \text { Biso } & 1.000 \mathrm{Pd} \\ \mathrm{Pd} 27 & 1.0000 & 0.00202 & 0.83340 & 0.32230 & \text { Biso } & 1.000 \mathrm{Pd} \\ \mathrm{Pd} 28 & 1.0000 & 0.66998 & 0.00006 & 0.28273 & \text { Biso } & 1.000 \mathrm{Pd} \\ \mathrm{Pd} 29 & 1.0000 & 0.66998 & 0.33340 & 0.28273 & \text { Biso } & 1.000 \mathrm{Pd} \\ \mathrm{Pd} 30 & 1.0000 & 0.66998 & 0.66673 & 0.28273 & \text { Biso } & 1.000 \mathrm{Pd} \\ \mathrm{Pd} 31 & 1.0000 & 0.33931 & 0.16673 & 0.25303 & \text { Biso } & 1.000 \mathrm{Pd} \\ \mathrm{Pd} 32 & 1.0000 & 0.33931 & 0.50006 & 0.25303 & \text { Biso } & 1.000 \mathrm{Pd} \\ \mathrm{Pd} 33 & 1.0000 & 0.33931 & 0.83340 & 0.25303 & \text { Biso } & 1.000 \mathrm{Pd} \\ \mathrm{Pd} 34 & 1.0000 & 0.00766 & 0.00006 & 0.22292 & \text { Biso } & 1.000 \mathrm{Pd} \\ \mathrm{Pd} 35 & 1.0000 & 0.00766 & 0.33340 & 0.22292 & \text { Biso } & 1.000 \mathrm{Pd} \\ \mathrm{Pd} 36 & 1.0000 & 0.00766 & 0.66673 & 0.22292 & \text { Biso } & 1.000 \mathrm{Pd} \\ \mathrm{O} 1 & 1.0000 & 0.66018 & 0.55400 & 0.69420 & \text { Biso } & 1.000 \mathrm{O} \\ \mathrm{C} 1 & 1.0000 & 0.26424 & 0.32732 & 0.65369 & \text { Biso } & 1.000 \mathrm{C} \\ \mathrm{C} 2 & 1.0000 & 0.17731 & 0.48407 & 0.67202 & \text { Biso } & 1.000 \mathrm{C} \\ \mathrm{C} 3 & 1.0000 & 0.14225 & 0.18564 & 0.67056 & \text { Biso } & 1.000 \mathrm{C} \\ \mathrm{H} 1 & 1.0000 & 0.41299 & 0.31523 & 0.66915 & \text { Biso } & 1.000 \mathrm{H} \\ \mathrm{H} 2 & 1.0000 & 0.79310 & 0.54761 & 0.70700 & \text { Biso } & 1.000 \mathrm{H} \\ \mathrm{H} 3 & 1.0000 & 0.26946 & 0.58538 & 0.66179 & \text { Biso } & 1.000 \mathrm{H} \\ \mathrm{H} 4 & 1.0000 & 0.20633 & 0.07299 & 0.65752 & \text { Biso } & 1.000 \mathrm{H} \\ \mathrm{H} 5 & 1.0000 & 0.03574 & 0.50360 & 0.65269 & \text { Biso } & 1.000 \mathrm{H} \\ \mathrm{H} 6 & 1.0000 & 0.15948 & 0.48271 & 0.71774 & \text { Biso } & 1.000 \mathrm{H} \\ \mathrm{H} 7 & 1.0000 & 0.13064 & 0.18334 & 0.71638 & \text { Biso } & 1.000 \mathrm{H} \\ \mathrm{H} 8 & 1.0000 & 0.99472 & 0.19185 & 0.65372 & \text { Biso } & 1.000 \mathrm{H} \\ & 1.0000 & 0.63473 & 0.66663 & 0.69062 & \text { Biso } & 1.000 \mathrm{H}\end{array}$

\# Pd111 E $=-60.05 ; \mathrm{ZPVE}=\mathrm{eV}$; frequencies $=\mathrm{cm}^{\wedge}-1$

data image 0

_cell_length_a 8.43904

_cell_length_b 8.43904

_cell_length_c 21.7

_cell_angle_alpha 90

_cell_angle_beta 90

_cell_angle_gamma 60

_symmetry_space_group_name_H-M "P 1"

_symmetry_int_tables_number 1

loop_

_symmetry_equiv_pos_as_xyz

'x, y, z'

loop_

_atom_site_label

_atom_site_occupancy

_atom_site_fract_x 


\begin{tabular}{|c|c|c|c|c|c|}
\hline \multicolumn{6}{|c|}{$\begin{array}{l}\text { atom_site_fract_y } \\
\text { _atom_site_fract_z } \\
\text { _atom_site_thermal_displace_type } \\
\text { atom_site_B_iso_or_equiv } \\
\text { atom_site_type_symbol }\end{array}$} \\
\hline Pd1 & 1.00001 .00000 & 0.97735 & 0.21086 & Biso & $1.000 \mathrm{Pd}$ \\
\hline $\mathrm{Pd} 2$ & 1.00000 .33333 & 0.97735 & 0.21086 & Biso & $1.000 \mathrm{Pd}$ \\
\hline $\mathrm{Pd} 3$ & 1.00000 .66666 & 0.97735 & 0.21086 & Biso & $1.000 \mathrm{Pd}$ \\
\hline $\mathrm{Pd} 4$ & 1.00001 .00000 & 0.31069 & 0.21086 & Biso & $1.000 \mathrm{Pd}$ \\
\hline $\mathrm{Pd} 5$ & 1.00000 .33332 & 0.31068 & 0.21085 & Biso & $1.000 \mathrm{Pd}$ \\
\hline Pd6 & 1.00000 .66667 & 0.31068 & 0.21085 & Biso & $1.000 \mathrm{Pd}$ \\
\hline $\operatorname{Pd} 7$ & 1.00001 .00000 & 0.64402 & 0.21086 & Biso & $1.000 \mathrm{Pd}$ \\
\hline $\operatorname{Pd} 8$ & 1.00000 .33332 & 0.64403 & 0.21085 & Biso & $1.000 \mathrm{Pd}$ \\
\hline Pd9 & 1.00000 .66666 & 0.64402 & 0.21086 & Biso & $1.000 \mathrm{Pd}$ \\
\hline $\operatorname{Pd} 10$ & 1.00000 .11111 & 0.08846 & 0.31734 & Biso & $1.000 \mathrm{Pd}$ \\
\hline Pd11 & 1.00000 .44444 & 0.08847 & 0.31734 & Biso & $1.000 \mathrm{Pd}$ \\
\hline Pd12 & 1.00000 .77777 & 0.08847 & 0.31734 & Biso & $1.000 \mathrm{Pd}$ \\
\hline $\operatorname{Pd} 13$ & 1.00000 .11112 & 0.42179 & 0.31734 & Biso & $1.000 \mathrm{Pd}$ \\
\hline $\operatorname{Pd} 14$ & 1.00000 .44444 & 0.42180 & 0.31732 & Biso & $1.000 \mathrm{Pd}$ \\
\hline Pd15 & 1.00000 .77777 & 0.42179 & 0.31734 & Biso & $1.000 \mathrm{Pd}$ \\
\hline Pd16 & 1.00000 .11112 & 0.75513 & 0.31734 & Biso & $1.000 \mathrm{Pd}$ \\
\hline Pd17 & 1.00000 .44444 & 0.75513 & 0.31734 & Biso & $1.000 \mathrm{Pd}$ \\
\hline Pd18 & 1.00000 .77777 & 0.75513 & 0.31734 & Biso & $1.000 \mathrm{Pd}$ \\
\hline Pd19 & 1.00000 .88894 & 0.19954 & 0.42267 & Biso & $1.000 \mathrm{Pd}$ \\
\hline $\operatorname{Pd} 20$ & 1.00000 .22223 & 0.19954 & 0.42265 & Biso & $1.000 \mathrm{Pd}$ \\
\hline $\operatorname{Pd} 21$ & 1.00000 .55556 & 0.19954 & 0.42266 & Biso & $1.000 \mathrm{Pd}$ \\
\hline $\operatorname{Pd} 22$ & 1.00000 .88893 & 0.53284 & 0.42266 & Biso & $1.000 \mathrm{Pd}$ \\
\hline $\operatorname{Pd} 23$ & 1.00000 .22226 & 0.53285 & 0.42266 & Biso & $1.000 \mathrm{Pd}$ \\
\hline $\mathrm{Pd} 24$ & 1.00000 .55558 & 0.53286 & 0.42266 & Biso & $1.000 \mathrm{Pd}$ \\
\hline $\operatorname{Pd} 25$ & 1.00000 .88892 & 0.86618 & 0.42266 & Biso & $1.000 \mathrm{Pd}$ \\
\hline $\operatorname{Pd} 26$ & 1.00000 .22226 & 0.86619 & 0.42266 & Biso & $1.000 \mathrm{Pd}$ \\
\hline $\operatorname{Pd} 27$ & 1.00000 .55558 & 0.86620 & 0.42266 & Biso & $1.000 \mathrm{Pd}$ \\
\hline $\operatorname{Pd} 28$ & 1.00000 .00003 & 0.97722 & 0.52919 & Biso & $1.000 \mathrm{Pd}$ \\
\hline $\operatorname{Pd} 29$ & 1.00000 .33338 & 0.97717 & 0.52918 & Biso & $1.000 \mathrm{Pd}$ \\
\hline $\operatorname{Pd} 30$ & 1.00000 .66668 & 0.97721 & 0.52920 & Biso & $1.000 \mathrm{Pd}$ \\
\hline $\operatorname{Pd} 31$ & 1.00000 .99996 & 0.31051 & 0.52914 & Biso & $1.000 \mathrm{Pd}$ \\
\hline Pd32 & 1.00000 .33344 & 0.31052 & 0.52916 & Biso & $1.000 \mathrm{Pd}$ \\
\hline $\mathrm{Pd} 33$ & 1.00000 .66669 & 0.31054 & 0.52918 & Biso & $1.000 \mathrm{Pd}$ \\
\hline $\mathrm{Pd} 34$ & 1.00000 .00000 & 0.64393 & 0.52918 & Biso & $1.000 \mathrm{Pd}$ \\
\hline Pd35 & 1.00000 .33337 & 0.64387 & 0.52920 & Biso & $1.000 \mathrm{Pd}$ \\
\hline $\operatorname{Pd} 36$ & 1.00000 .66668 & 0.64387 & 0.52919 & Biso & $1.000 \mathrm{Pd}$ \\
\hline
\end{tabular}

\# Pd111.Ad.O E= -64.39; ZPVE=0.07 eV; frequencies=397.33, 325.84, $325.79 \mathrm{~cm}^{\wedge}-1$

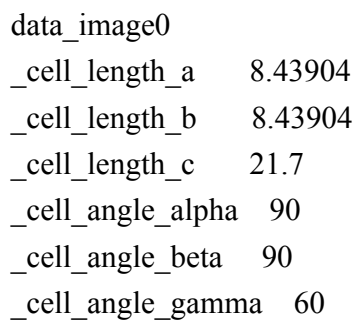




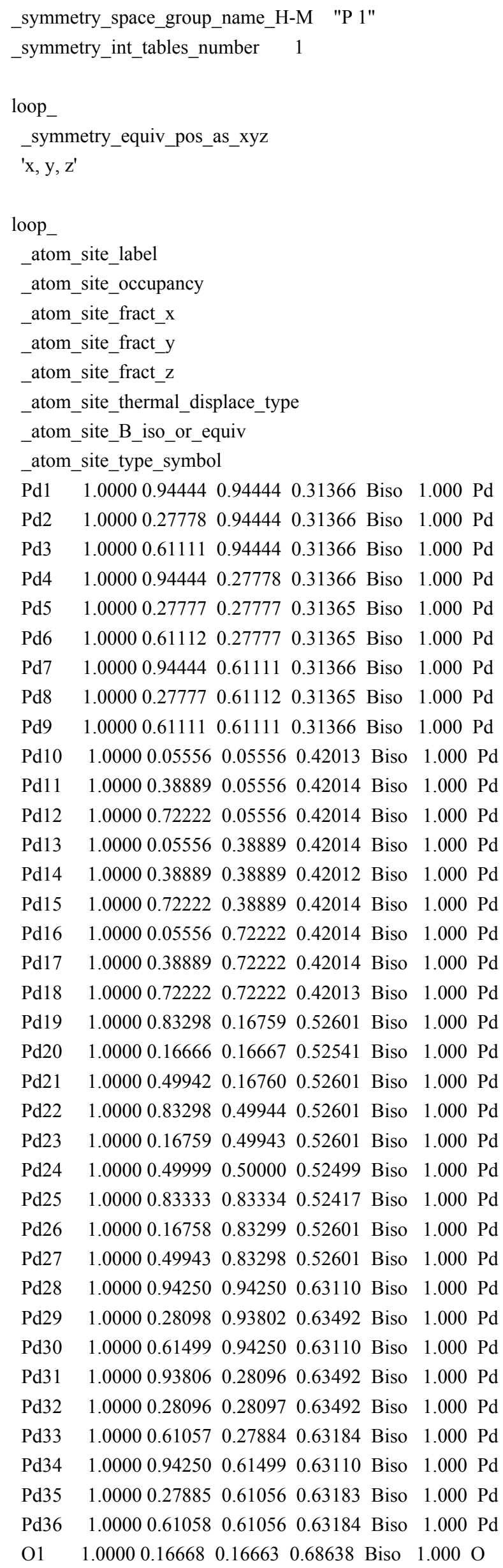


\# Pd111.Ad.OH E= -68.64; ZPVE= $0.35 \mathrm{eV}$; frequencies $=3685.61,627.73,600.21,340.25,202.00,123.08 \mathrm{~cm}^{\wedge}-1$

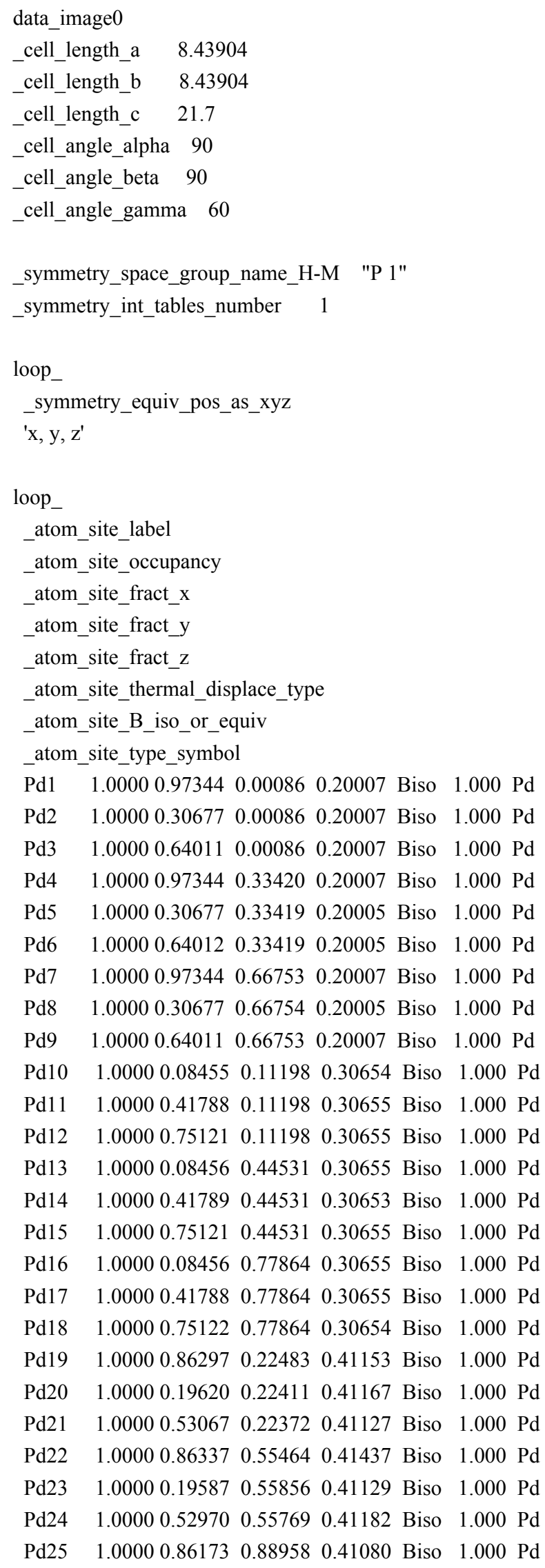




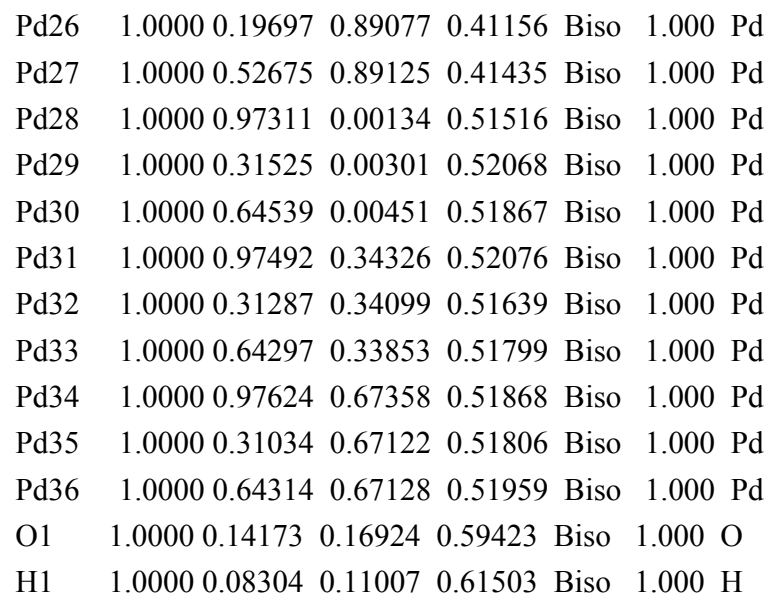




\begin{tabular}{|c|c|c|c|c|c|}
\hline Pd13 & $1.0000 \quad 0.32989$ & 0.16667 & 0.23250 & Biso & \\
\hline Pd14 & 1.00000 .32989 & 0.50000 & 0.23250 & Biso & 1.000 \\
\hline $\operatorname{Pd} 15$ & 1.00000 .32989 & 0.83333 & 0.23250 & Biso & \\
\hline $\operatorname{Pd} 16$ & 1.00000 .99930 & 1.00000 & 0.19990 & Biso & 1.000 \\
\hline Pd17 & 1.00000 .99930 & 0.33333 & 0.19990 & Biso & 1.000 \\
\hline Pd18 & 1.00000 .99930 & 0.66667 & 0.19990 & Biso & 1.000 \\
\hline Pd19 & 1.00000 .66736 & 0.16667 & 0.16408 & Biso & 1.000 \\
\hline $\operatorname{Pd} 20$ & 1.00000 .66736 & 0.50000 & 0.16408 & Biso & 1.000 \\
\hline $\operatorname{Pd} 21$ & 1.00000 .66736 & 0.83333 & 0.16408 & Biso & 1.000 \\
\hline $\operatorname{Pd} 22$ & 1.00000 .33677 & 1.00000 & 0.13148 & Biso & 1.000 \\
\hline $\operatorname{Pd} 23$ & 1.00000 .33677 & 0.33333 & 0.13148 & Biso & 1.000 \\
\hline $\mathrm{Pd} 24$ & 1.00000 .33677 & 0.66667 & 0.13148 & Biso & 1.000 \\
\hline $\operatorname{Pd} 25$ & 1.00000 .00041 & 0.16667 & 0.09945 & Biso & 1.000 \\
\hline $\operatorname{Pd} 26$ & 1.00000 .00041 & 0.50000 & 0.09945 & Biso & 1.00 \\
\hline $\operatorname{Pd} 27$ & 1.00000 .00041 & 0.83333 & 0.09945 & Biso & 1.000 \\
\hline $\operatorname{Pd} 28$ & 1.00000 .66837 & 1.00000 & 0.05988 & Biso & 1.000 \\
\hline $\operatorname{Pd} 29$ & 1.00000 .66837 & 0.33333 & 0.05988 & Biso & 1.000 \\
\hline $\operatorname{Pd} 30$ & 1.00000 .66837 & 0.66667 & 0.05988 & Biso & 1.000 \\
\hline $\operatorname{Pd} 31$ & 1.00000 .33770 & 0.16667 & 0.03018 & Biso & 1.000 \\
\hline $\operatorname{Pd} 32$ & 1.00000 .33770 & 0.50000 & 0.03018 & Biso & 1.000 \\
\hline $\mathrm{Pd} 33$ & 1.00000 .33770 & 0.83333 & 0.03018 & Biso & 1.000 \\
\hline $\mathrm{Pd} 34$ & 1.00000 .00606 & 1.00000 & 0.00008 & Biso & 1.000 \\
\hline $\operatorname{Pd} 35$ & 1.00000 .00606 & 0.33333 & 0.00008 & Biso & 1.000 \\
\hline $\operatorname{Pd} 36$ & 1.00000 .00606 & 0.6666 & 0.00008 & Biso & 1.000 \\
\hline
\end{tabular}

\# Pd211.Ad.O E= -63.99; ZPVE=0.07 eV; frequencies= 404.89, 349.97, $344.31 \mathrm{~cm}^{\wedge}-1$

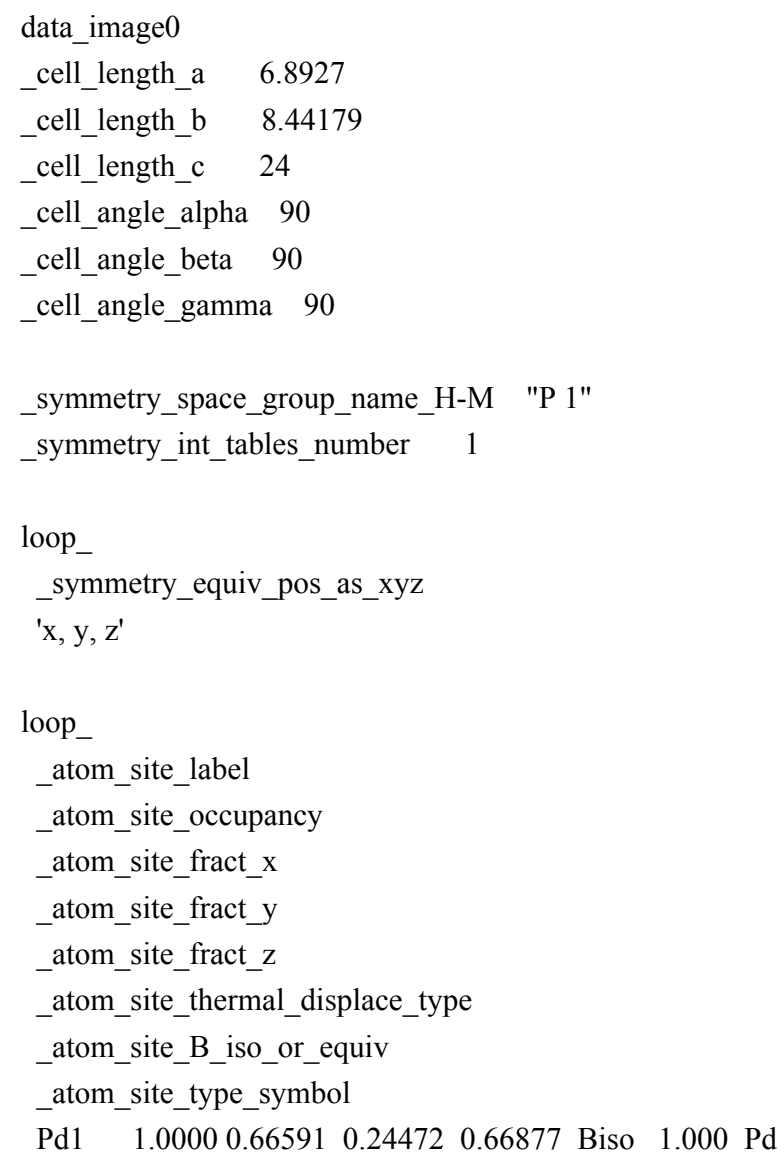




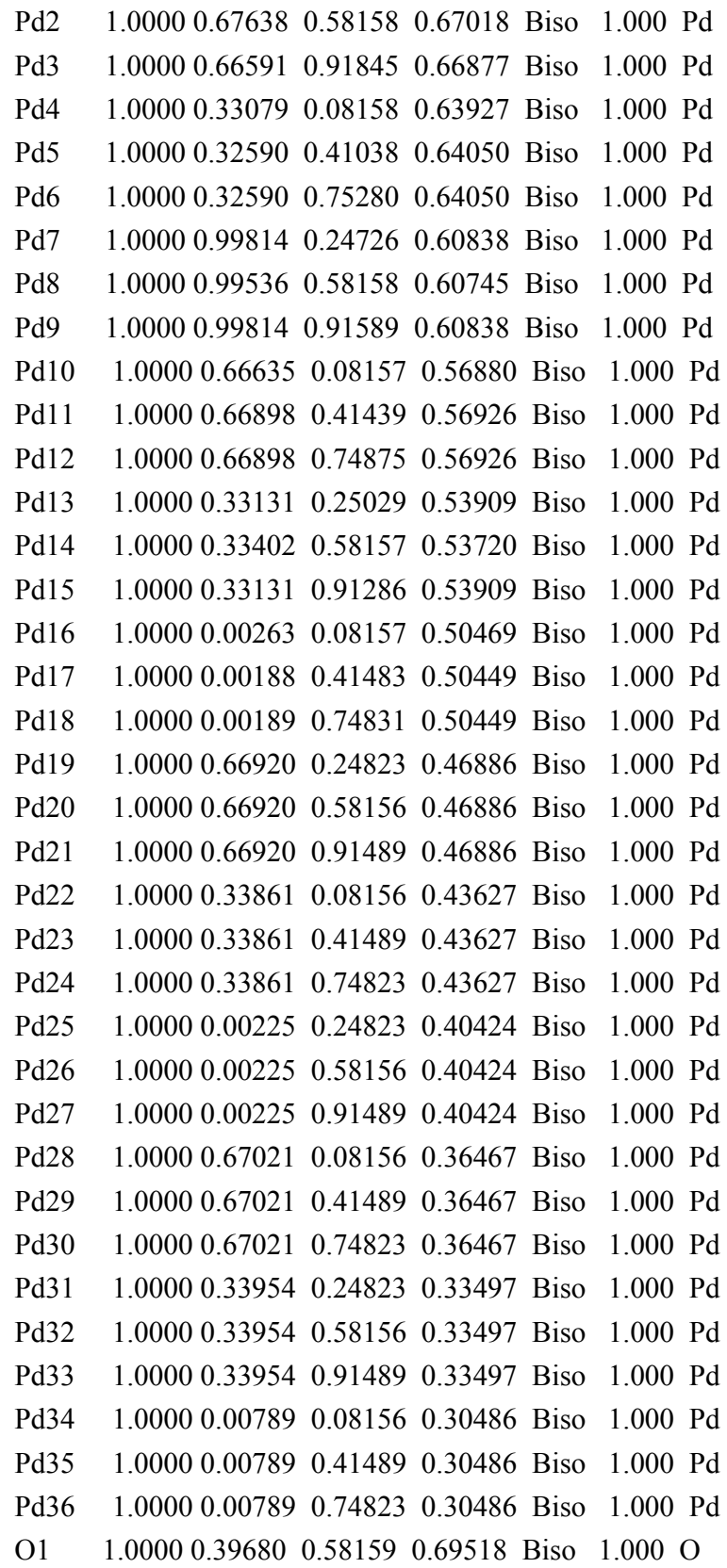




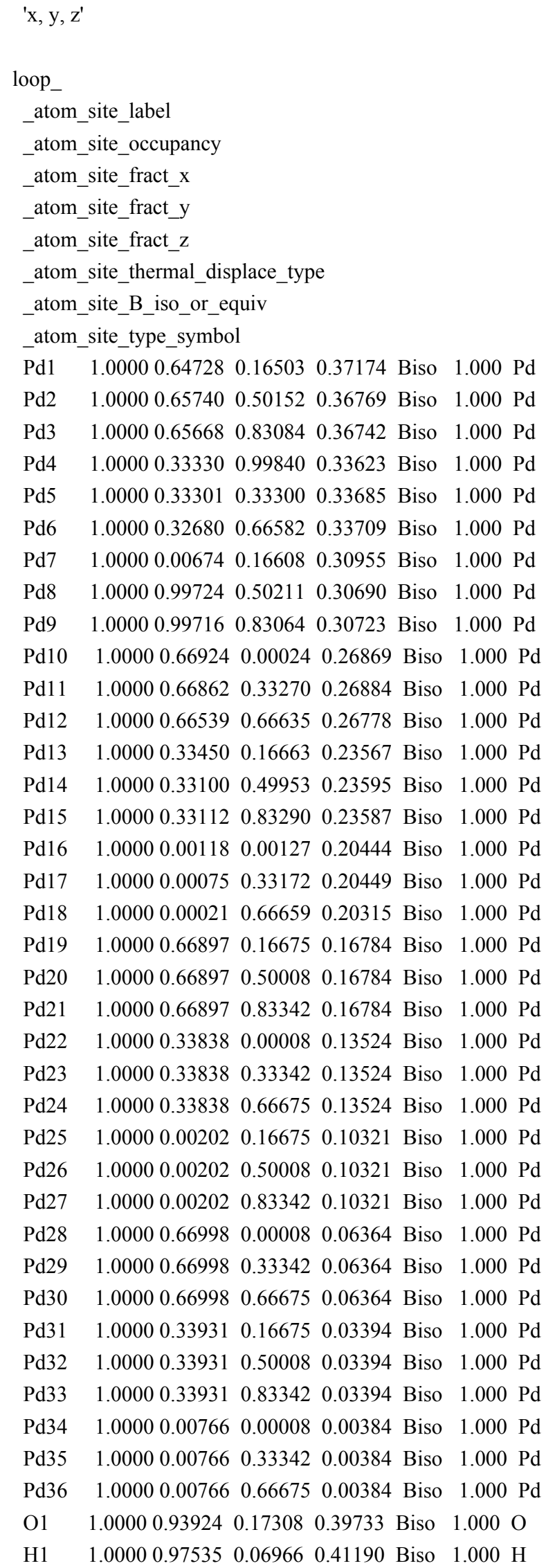

\# Pd111(4x4) E= -106.60; ZPVE $=\mathrm{eV}$; frequencies $=\mathrm{cm}^{\wedge}-1$ 


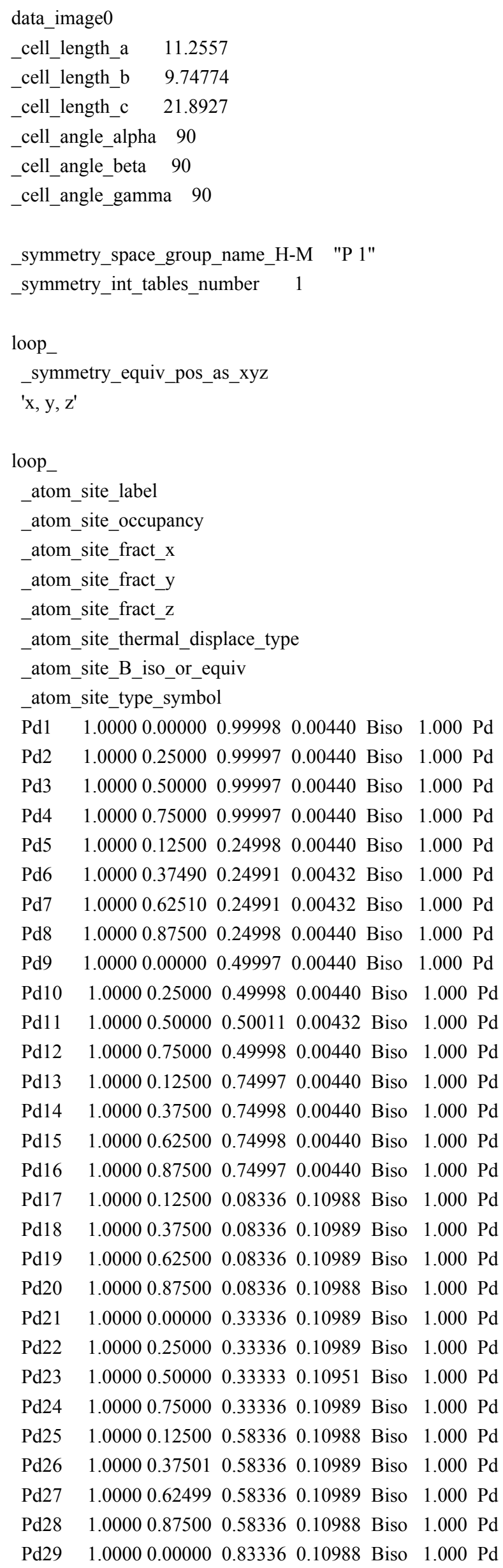




\begin{tabular}{|c|c|c|c|c|c|c|}
\hline $\operatorname{Pd} 30$ & 1.00000 .25000 & 0.83336 & 0.10989 & Biso & 1.000 & $\mathrm{Pd}$ \\
\hline $\operatorname{Pd} 31$ & 1.00000 .50000 & 0.83336 & 0.10989 & Biso & 1.000 & \\
\hline $\operatorname{Pd} 32$ & 1.00000 .75000 & 0.83336 & 0.10989 & Biso & 1.000 & $\mathrm{Pd}$ \\
\hline d33 & 1.00000 .00000 & 0.16664 & 0.21408 & Biso & 1.000 & $\mathrm{Pd}$ \\
\hline $\operatorname{Pd} 34$ & 1.00000 .25000 & 0.16664 & 0.21408 & Biso & 1.000 & $\mathrm{Pd}$ \\
\hline 135 & 1.00000 .50000 & 16677 & 0.21400 & Biso & 1.000 & $\mathrm{Pd}$ \\
\hline $\operatorname{Pd} 36$ & 1.00000 .75000 & 0.166 & & Biso & 1.000 & $\mathrm{Pd}$ \\
\hline $\operatorname{Pd} 37$ & 1.00000 .12500 & 0.41664 & 0.21408 & Biso & 1.000 & $\mathrm{Pd}$ \\
\hline $\operatorname{Pd} 38$ & 1.00000 .37510 & 0.41657 & 0.21400 & Biso & 1.000 & $\mathrm{Pd}$ \\
\hline $\mathrm{d} 39$ & 1.00000 .62490 & 0.41657 & 0.21400 & Biso & 1.000 & $\mathrm{Pd}$ \\
\hline$d 40$ & 500 & & & Biso & 1.000 & $\mathrm{Pd}$ \\
\hline$d 41$ & 1.00000 .00000 & 0.66664 & 0.21408 & Biso & 1.000 & $\mathrm{Pd}$ \\
\hline$d 42$ & 1.00000 .25000 & 0.66663 & 0.21408 & Biso & 1.000 & $\mathrm{Pd}$ \\
\hline $\mathrm{Pd} 43$ & 1.00000 .50000 & 0.66664 & 0.21408 & Biso & 1.000 & $\mathrm{Pd}$ \\
\hline Pd44 & 1.00000 .75000 & 0.66663 & 0.21408 & Biso & 1.000 & $\mathrm{Pd}$ \\
\hline $\operatorname{Pd} 45$ & 1.00000 .12500 & 0.91664 & 0.21408 & Biso & 1.000 & $\mathrm{Pd}$ \\
\hline Pd46 & 1.00000 .37500 & 0.91665 & 0.21408 & Biso & 1.000 & $\mathrm{Pd}$ \\
\hline 147 & 1.00000 .62500 & 0.91665 & 0.21408 & Biso & 1.000 & $\mathrm{Pd}$ \\
\hline Pd48 & 1.00000 .87500 & 0.91664 & 0.21408 & Biso & 1.000 & $\mathrm{Pd}$ \\
\hline Pd49 & 1.00000 .00000 & 0.00002 & 0.31959 & Biso & 1.000 & $\mathrm{Pd}$ \\
\hline $\operatorname{Pd} 50$ & 1.00000 .25000 & 0.00003 & 0.31959 & Biso & 1.000 & $\mathrm{Pd}$ \\
\hline Pd51 & 1.00000 .50000 & 0.00001 & 0.31959 & Biso & 1.000 & $\mathrm{Pd}$ \\
\hline Pd52 & 1.00000 .75000 & 0.00003 & 0.31959 & Biso & 1.000 & $\mathrm{Pd}$ \\
\hline Pd53 & 1.00000 .12500 & 0.25003 & 0.31959 & Biso & 1.000 & $\mathrm{Pd}$ \\
\hline Pd54 & 1.00000 .37500 & 0.25003 & 0.31958 & Biso & 1.000 & $\mathrm{Pd}$ \\
\hline Pd55 & 1.00000 .62500 & 0.25003 & 0.31958 & Biso & 1.000 & $\mathrm{Pd}$ \\
\hline Pd56 & 1.00000 .87500 & 0.25003 & 0.31959 & Biso & 1.000 & $\mathrm{Pd}$ \\
\hline Pd57 & 1.00000 .00000 & 0.50003 & 0.31959 & Biso & 1.000 & $\mathrm{Pd}$ \\
\hline $\operatorname{Pd} 58$ & 1.00000 .24999 & 0.50003 & 0.31959 & Biso & 1.000 & $\mathrm{Pd}$ \\
\hline $\operatorname{Pd} 59$ & 1.00000 .50000 & 0.50002 & 0.31958 & Biso & 1.000 & $\mathrm{Pd}$ \\
\hline Pd60 & 1.00000 .75001 & 0.50003 & 0.31959 & Biso & 1.000 & $\mathrm{Pd}$ \\
\hline Pd61 & 1.00000 .12500 & 0.75002 & 0.31959 & Biso & 1.000 & $\mathrm{Pd}$ \\
\hline Pd62 & 1.00000 .37500 & 0.75002 & 0.31959 & Biso & 1.000 & $\mathrm{Pd}$ \\
\hline Pd63 & 1.00000 .62500 & 0.75002 & 0.31959 & Biso & 1.000 & $\mathrm{Pd}$ \\
\hline Pd64 & 1.00000 .87500 & 0.75002 & 0.31959 & Biso & 1.000 & D \\
\hline
\end{tabular}

\# Pd111(4x4).A1.P E= -160.95; ZPVE $=\mathrm{eV}$; frequencies $=\mathrm{cm}^{\wedge}-1$

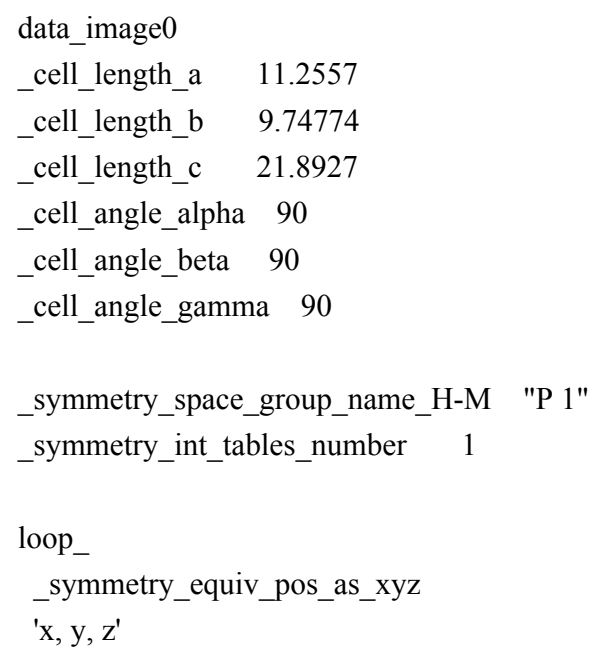




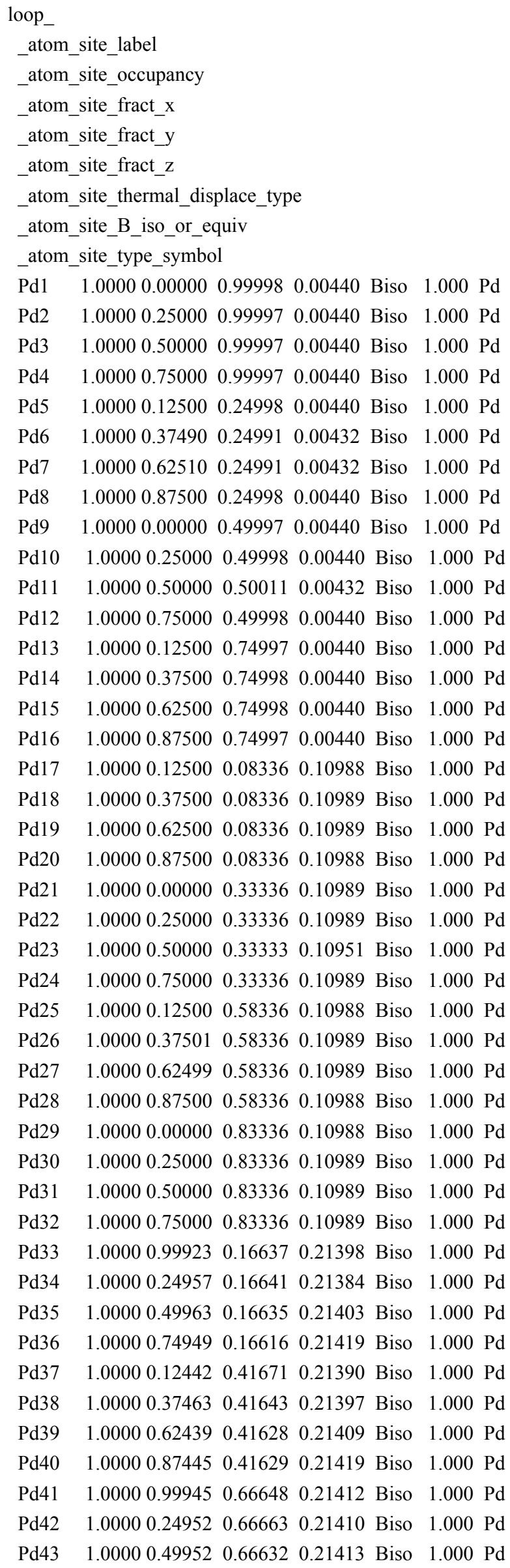




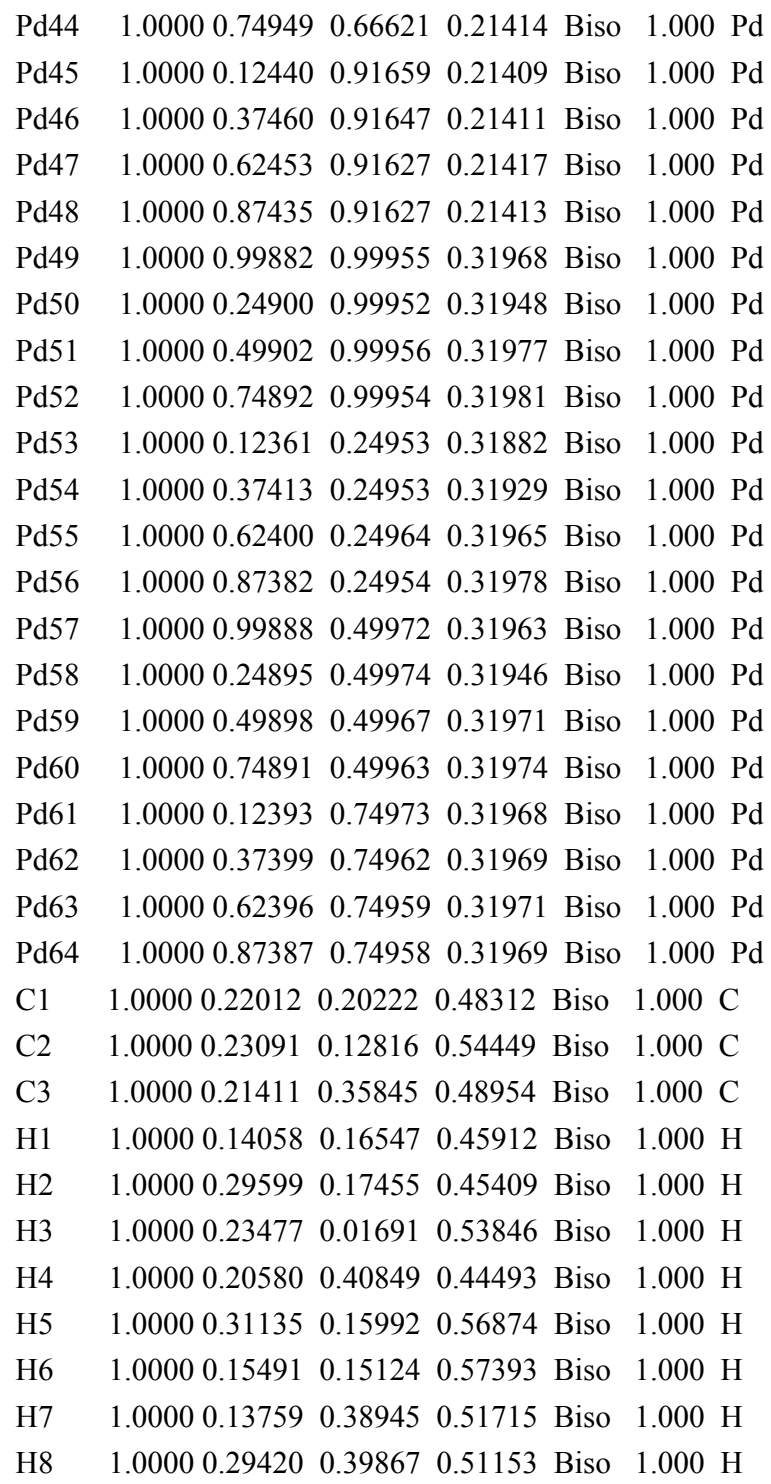

\# Pd111(4x4).A1.T1 E= -159.97; ZPVE $=\mathrm{eV}$; frequencies $=\mathrm{cm}^{\wedge}-1$

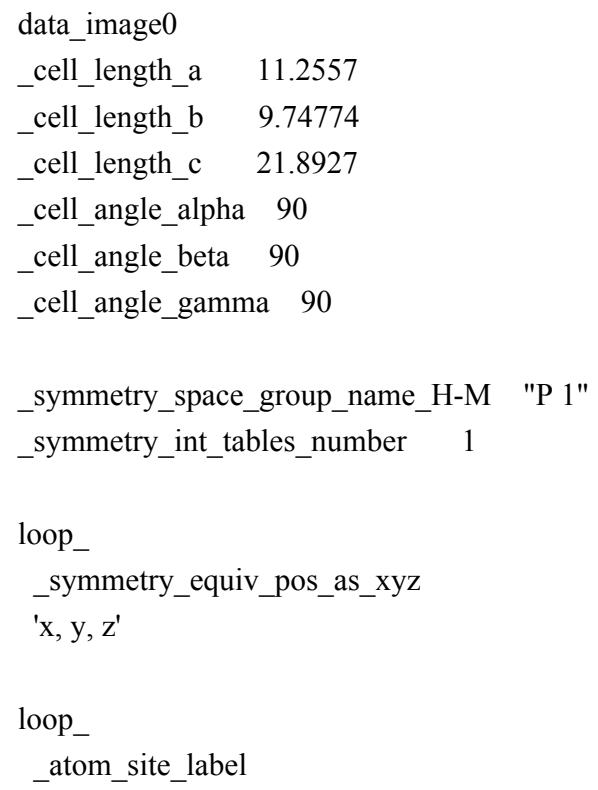




\begin{tabular}{|c|c|c|c|c|c|c|}
\hline \multicolumn{7}{|c|}{$\begin{array}{l}\text {-atom_site_occupancy } \\
\text {-atom_site_fract_x } \\
\text {-atom_site_fract_y } \\
\text {-atom_site_fract_z } \\
\text { atom_site_thermal_displace_type } \\
\text {-atom_site_B_iso_or_equiv } \\
\text { atom_site_type_symbol }\end{array}$} \\
\hline Pd1 & 1.00000 .00000 & 0.99998 & 0.00440 & Biso & $1.000 \mathrm{~F}$ & $\mathrm{Pd}$ \\
\hline $\operatorname{Pd} 2$ & 1.00000 .25000 & 0.99997 & 0.00440 & Biso & $1.000 \mathrm{~F}$ & $\mathrm{Pd}$ \\
\hline $\mathrm{Pd} 3$ & 1.00000 .50000 & 0.99997 & 0.00440 & Biso & $1.000 \mathrm{~F}$ & $\mathrm{Pd}$ \\
\hline Pd4 & 1.00000 .75000 & 0.99997 & 0.00440 & Biso & $1.000 \mathrm{~F}$ & $\mathrm{Pd}$ \\
\hline $\mathrm{Pd} 5$ & 1.00000 .12500 & 0.24998 & 0.00440 & Biso & $1.000 \mathrm{~F}$ & $\mathrm{Pd}$ \\
\hline Pd6 & 1.00000 .37490 & 0.24991 & 0.00432 & Biso & $1.000 \mathrm{~F}$ & $\mathrm{Pd}$ \\
\hline $\operatorname{Pd} 7$ & 1.00000 .62510 & 0.24991 & 0.00432 & Biso & $1.000 \mathrm{~F}$ & $\mathrm{Pd}$ \\
\hline $\operatorname{Pd} 8$ & 1.00000 .87500 & 0.24998 & 0.00440 & Biso & $1.000 \mathrm{~F}$ & $\mathrm{Pd}$ \\
\hline Pd9 & 1.00000 .00000 & 0.49997 & 0.00440 & Biso & $1.000 \mathrm{~F}$ & $\mathrm{Pd}$ \\
\hline $\operatorname{Pd} 10$ & 1.00000 .25000 & 0.4999 & 0.00440 & Biso & 1.000 & $\mathrm{Pd}$ \\
\hline $\operatorname{Pd} 11$ & 1.00000 .50000 & 0.5001 & 0.00432 & Biso & 1.000 & $\mathrm{Pd}$ \\
\hline $\operatorname{Pd} 12$ & 1.00000 .75000 & 0.49998 & 0.00440 & Biso & 1.000 & $\mathrm{Pd}$ \\
\hline Pd13 & 1.00000 .12500 & 0.74997 & 0.00440 & Biso & 1.000 & $\mathrm{Pd}$ \\
\hline Pd14 & 1.00000 .37500 & 0.74998 & 0.00440 & Biso & 1.000 & $\mathrm{Pd}$ \\
\hline $\operatorname{Pd} 15$ & 1.00000 .62500 & 0.74998 & 0.00440 & Biso & 1.000 & $\mathrm{Pd}$ \\
\hline Pd16 & 1.00000 .87500 & 0.74997 & 0.00440 & Biso & 1.000 & $\mathrm{Pd}$ \\
\hline $\operatorname{Pd} 17$ & 1.00000 .12500 & 0.08336 & 0.10988 & Biso & 1.000 & $\mathrm{Pd}$ \\
\hline $\operatorname{Pd} 18$ & 1.00000 .37500 & 0.08336 & 0.10989 & Biso & 1.000 & $\mathrm{Pd}$ \\
\hline Pd19 & 1.00000 .62500 & 0.08336 & 0.10989 & Biso & 1.000 & $\mathrm{Pd}$ \\
\hline $\operatorname{Pd} 20$ & 1.00000 .87500 & 0.08336 & 0.10988 & Biso & 1.000 & $\mathrm{Pd}$ \\
\hline $\operatorname{Pd} 21$ & 1.00000 .00000 & 0.33336 & 0.10989 & Biso & 1.000 & $\mathrm{Pd}$ \\
\hline $\operatorname{Pd} 22$ & 1.00000 .25000 & 0.33336 & 0.10989 & Biso & 1.000 & $\mathrm{Pd}$ \\
\hline $\operatorname{Pd} 23$ & 1.00000 .50000 & 0.33333 & 0.10951 & Biso & 1.000 & $\mathrm{Pd}$ \\
\hline $\operatorname{Pd} 24$ & 1.00000 .75000 & 0.33336 & 0.10989 & Biso & 1.000 & $\mathrm{Pd}$ \\
\hline $\operatorname{Pd} 25$ & 1.00000 .12500 & 0.58336 & 0.10988 & Biso & 1.000 & $\mathrm{Pd}$ \\
\hline $\operatorname{Pd} 26$ & 1.00000 .37501 & 0.58336 & 0.10989 & Biso & 1.000 & $\mathrm{Pd}$ \\
\hline $\operatorname{Pd} 27$ & 1.00000 .62499 & 0.58336 & 0.10989 & Biso & 1.000 & $\mathrm{Pd}$ \\
\hline $\operatorname{Pd} 28$ & 1.00000 .87500 & 0.58336 & 0.10988 & Biso & 1.000 & $\mathrm{Pd}$ \\
\hline $\operatorname{Pd} 29$ & 1.00000 .00000 & 0.83336 & 0.10988 & Biso & 1.000 & $\mathrm{Pd}$ \\
\hline $\mathrm{Pd} 30$ & 1.00000 .25000 & 0.83336 & 0.10989 & Biso & 1.000 & $\mathrm{Pd}$ \\
\hline Pd31 & 1.00000 .50000 & 0.83336 & 0.10989 & Biso & 1.000 & $\mathrm{Pd}$ \\
\hline $\operatorname{Pd} 32$ & 1.00000 .75000 & 0.83336 & 0.10989 & Biso & 1.000 & $\mathrm{Pd}$ \\
\hline $\operatorname{Pd} 33$ & 1.00000 .99907 & 0.16622 & 0.21400 & Biso & 1.000 & $\mathrm{Pd}$ \\
\hline $\mathrm{Pd} 34$ & 1.00000 .25007 & 0.16636 & 0.21394 & Biso & 1.000 & $\mathrm{Pd}$ \\
\hline $\operatorname{Pd} 35$ & 1.00000 .50246 & 0.16872 & 0.21677 & Biso & 1.000 & $\mathrm{Pd}$ \\
\hline Pd36 & 1.00000 .74916 & 0.16687 & 0.21502 & Biso & 1.000 & $\mathrm{Pd}$ \\
\hline $\operatorname{Pd} 37$ & 1.00000 .12437 & 0.41615 & 0.21384 & Biso & 1.000 & $\mathrm{Pd}$ \\
\hline $\operatorname{Pd} 38$ & 1.00000 .37401 & 0.41620 & 0.21355 & Biso & 1.000 & $\mathrm{Pd}$ \\
\hline $\operatorname{Pd} 39$ & 1.00000 .62487 & 0.41610 & 0.21503 & Biso & 1.000 & $\mathrm{Pd}$ \\
\hline $\operatorname{Pd} 40$ & 1.00000 .87475 & 0.41658 & 0.21421 & Biso & 1.000 & $\mathrm{Pd}$ \\
\hline $\operatorname{Pd} 41$ & 1.00000 .99976 & 0.66647 & 0.21401 & Biso & 1.000 & $\mathrm{Pd}$ \\
\hline $\operatorname{Pd} 42$ & 1.00000 .24936 & 0.66662 & 0.21390 & Biso & 1.000 & $\mathrm{Pd}$ \\
\hline $\operatorname{Pd} 43$ & 1.00000 .49914 & 0.66657 & 0.21351 & Biso & 1.000 & $\mathrm{Pd}$ \\
\hline Pd44 & 1.00000 .74954 & 0.66510 & 0.21431 & Biso & 1.000 & $\mathrm{Pd}$ \\
\hline $\operatorname{Pd} 45$ & 1.00000 .12464 & 0.91668 & 0.21396 & Biso & 1.000 & $\mathrm{Pd}$ \\
\hline Pd46 & 1.00000 .37510 & 0.91734 & 0.21390 & Biso & 1.000 & $\mathrm{Pd}$ \\
\hline
\end{tabular}




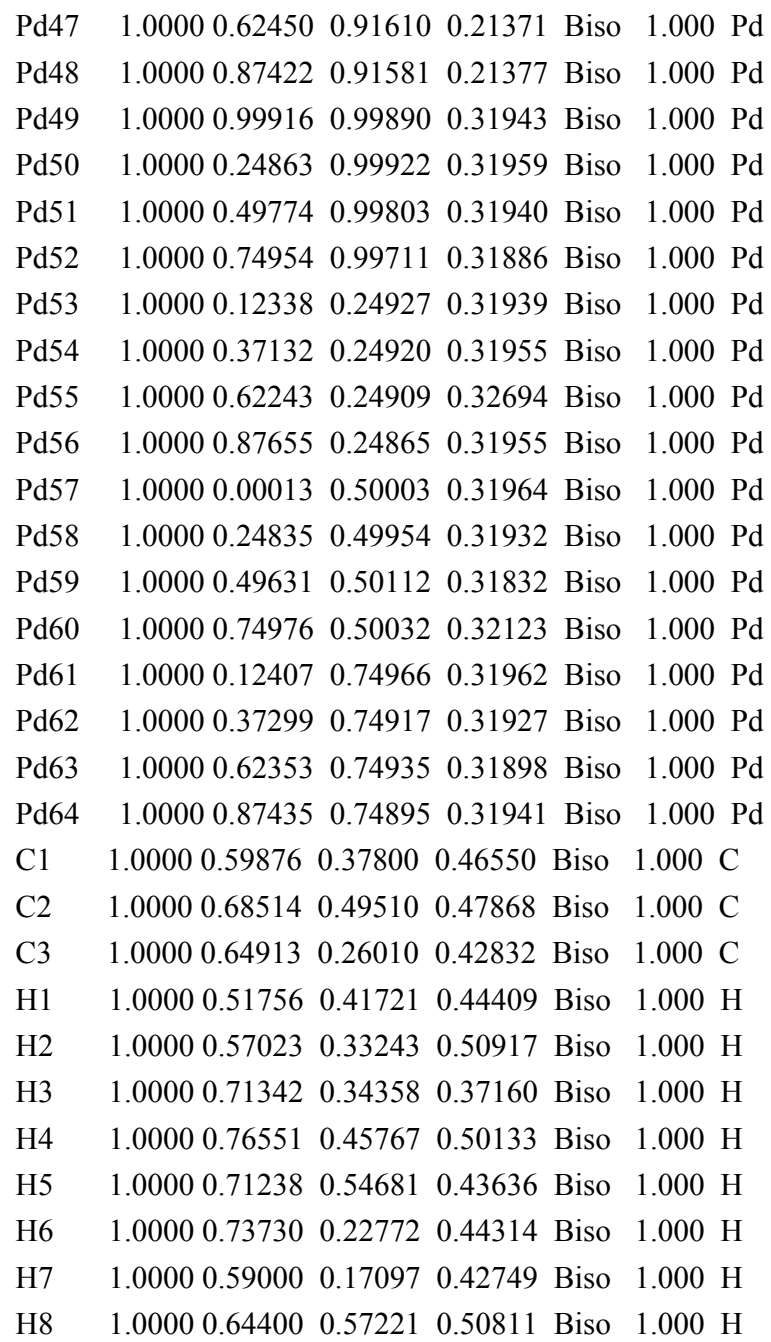




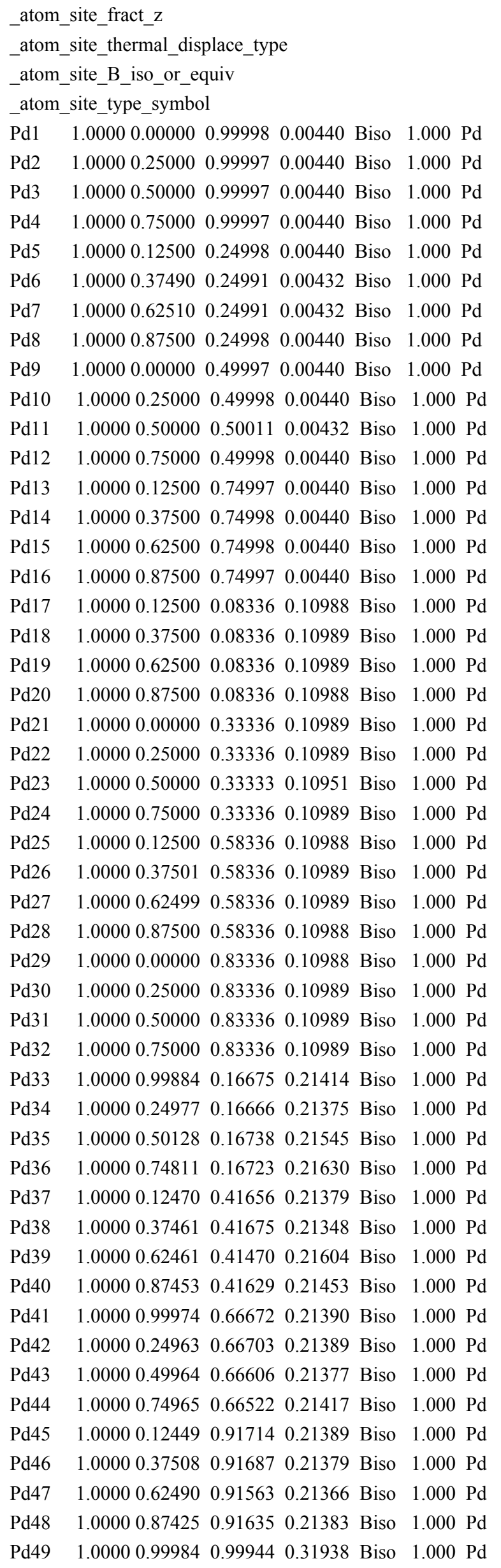




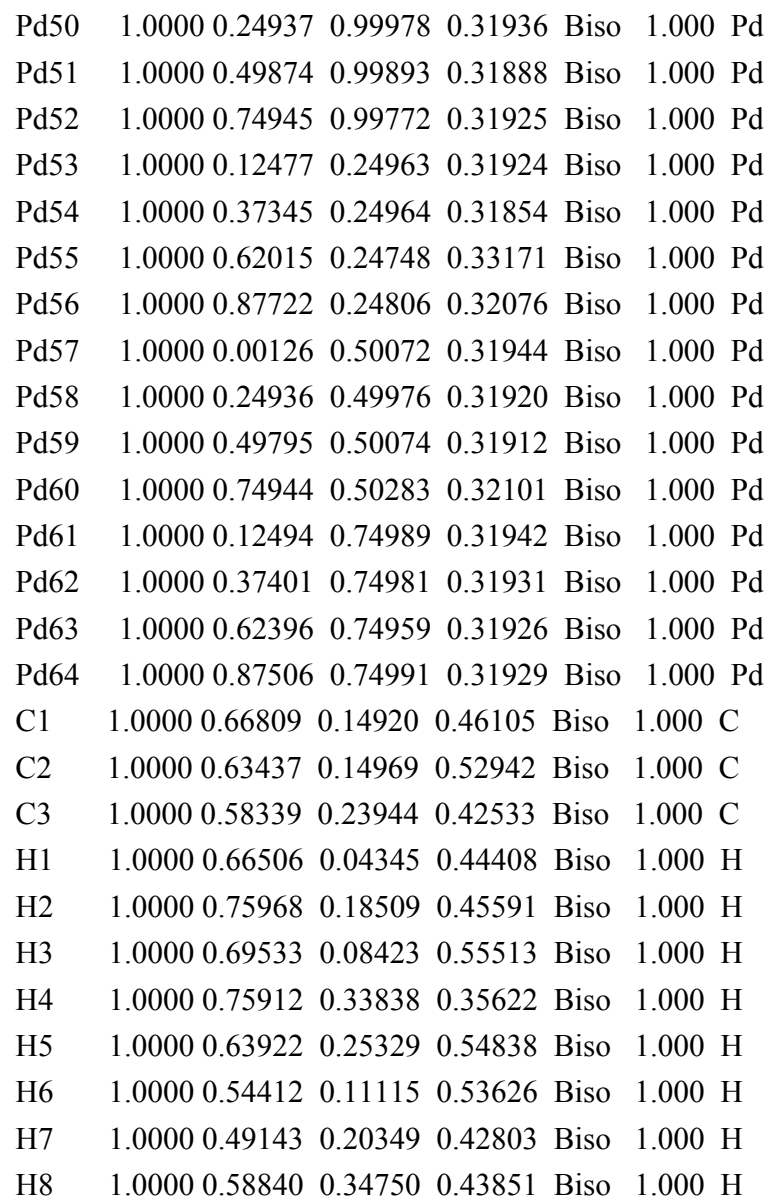

\# Pd111(4x4).Ads.OH E= -115.28; ZPVE $=\mathrm{eV}$; frequencies $=\mathrm{cm}^{\wedge}-1$

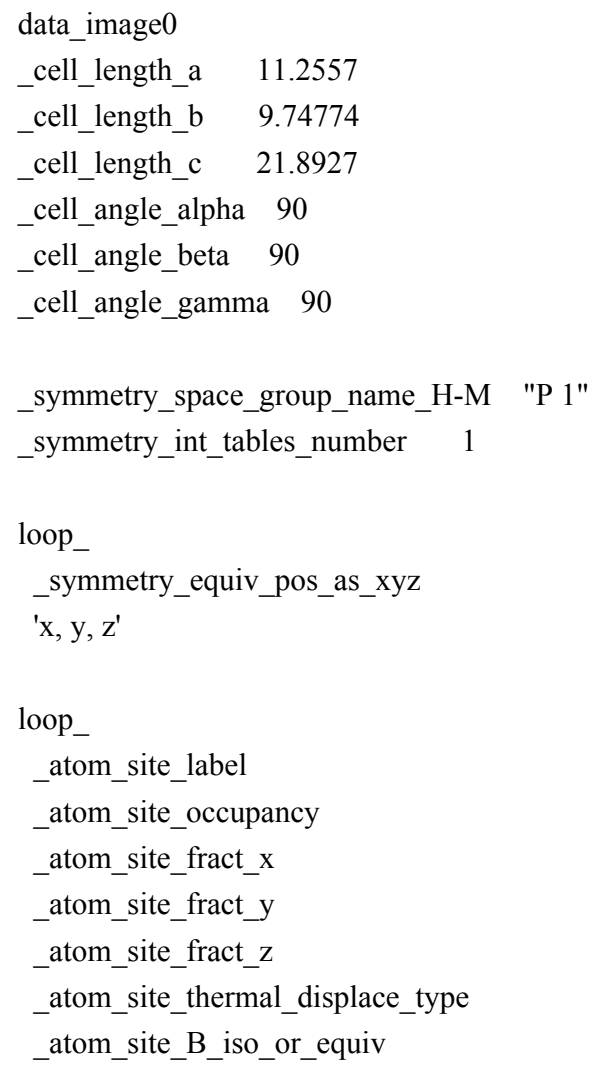




\begin{tabular}{|c|c|c|c|c|c|c|}
\hline Pd1 & 1.00000 .00000 & 0.99998 & 0.00440 & Biso & $1.000 \mathrm{I}$ & $\mathrm{Pd}$ \\
\hline $\mathrm{Pd} 2$ & 1.00000 .25000 & 0.99997 & 0.00440 & Biso & $1.000 \mathrm{I}$ & $\mathrm{Pd}$ \\
\hline $\mathrm{Pd} 3$ & 1.00000 .50000 & 0.99997 & 0.00440 & Biso & $1.000 \mathrm{I}$ & $\mathrm{Pd}$ \\
\hline $\mathrm{Pd} 4$ & 1.00000 .75000 & 0.99997 & 0.00440 & Biso & $1.000 \mathrm{I}$ & $\mathrm{Pd}$ \\
\hline Pd5 & 1.00000 .12500 & 0.24998 & 0.00440 & Biso & $1.000 \mathrm{I}$ & $\mathrm{Pd}$ \\
\hline Pd6 & 1.00000 .37490 & 0.24991 & 0.00432 & Biso & $1.000 \mathrm{I}$ & $\mathrm{Pd}$ \\
\hline $\operatorname{Pd} 7$ & 1.00000 .62510 & 0.24991 & 0.00432 & Biso & $1.000 \mathrm{I}$ & $\mathrm{Pd}$ \\
\hline $\mathrm{Pd} 8$ & 1.00000. & 0.24998 & 0.00440 & Biso & $1.000 \mathrm{I}$ & $\mathrm{Pd}$ \\
\hline Pd9 & 1.00000 .00000 & 0.49997 & 0.00440 & Biso & $1.000 \mathrm{I}$ & $\mathrm{Pd}$ \\
\hline $\operatorname{Pd} 10$ & 1.00000 .25000 & 0.49998 & 0.00440 & Biso & 1.000 & $\mathrm{Pd}$ \\
\hline $\operatorname{Pd} 11$ & 1.00000 .50000 & 0.50011 & 0.00432 & Biso & 1.000 & $\mathrm{Pd}$ \\
\hline $\operatorname{Pd} 12$ & 1.00000 .75000 & 0.49998 & 0.00440 & Biso & 1.000 & $\mathrm{Pd}$ \\
\hline $\operatorname{Pd} 13$ & & & 0.00440 & Biso & 1.000 & $\mathrm{Pd}$ \\
\hline $\operatorname{Pd} 14$ & 1.00000 .37500 & 0.74998 & 0.00440 & Biso & 1.000 & $\mathrm{Pd}$ \\
\hline $\operatorname{Pd} 15$ & 1.00000 .62500 & 0.74998 & 0.00440 & Biso & 1.000 & $\mathrm{Pd}$ \\
\hline Pd16 & 1.00000 .87500 & 0.74997 & 0.00440 & Biso & 1.000 & $\mathrm{Pd}$ \\
\hline $\operatorname{Pd} 17$ & 1.00000 .12500 & 0.08336 & 0.10988 & Biso & 1.000 & $\mathrm{Pd}$ \\
\hline $\operatorname{Pd} 18$ & 1.00000 .37500 & 0.08336 & 0.10989 & Biso & 1.000 & $\mathrm{Pd}$ \\
\hline Pd19 & 1.00000 .62500 & 0.08336 & 0.10989 & Biso & 1.000 & $\mathrm{Pd}$ \\
\hline $\operatorname{Pd} 20$ & 1.00000 .87500 & 0.08336 & 0.10988 & Biso & 1.000 & $\mathrm{Pd}$ \\
\hline $\operatorname{Pd} 21$ & 1.00000 .00000 & 0.33336 & 0.10989 & Biso & 1.000 & $\mathrm{Pd}$ \\
\hline $\operatorname{Pd} 22$ & 1.00000 .25000 & 0.33336 & 0.10989 & Biso & 1.000 & $\mathrm{Pd}$ \\
\hline $\operatorname{Pd} 23$ & 1.00000 .50000 & 0.33333 & 0.10951 & Biso & 1.000 & $\mathrm{Pd}$ \\
\hline $\operatorname{Pd} 24$ & 1.00000 .75000 & 0.33336 & 0.10989 & Biso & 1.000 & $\mathrm{Pd}$ \\
\hline $\operatorname{Pd} 25$ & 1.00000 .12500 & 0.58336 & 0.10988 & Biso & 1.000 & $\mathrm{Pd}$ \\
\hline $\operatorname{Pd} 26$ & 1.00000 .37501 & 0.58336 & 0.10989 & Biso & 1.000 & $\mathrm{Pd}$ \\
\hline $\operatorname{Pd} 27$ & 1.00000 .62499 & 0.58336 & 0.10989 & Biso & 1.000 & $\mathrm{Pd}$ \\
\hline $\operatorname{Pd} 28$ & 1.00000 .87500 & 0.58336 & 0.10988 & Biso & 1.000 & $\mathrm{Pd}$ \\
\hline $\operatorname{Pd} 29$ & 1.00000 .00000 & 0.83336 & 0.10988 & Biso & 1.000 & $\mathrm{Pd}$ \\
\hline $\mathrm{Pd} 30$ & 1.00000 .25000 & 0.83336 & 0.10989 & Biso & 1.000 & $\mathrm{Pd}$ \\
\hline $\operatorname{Pd} 31$ & 1.00000 .50000 & 0.83336 & 0.10989 & Biso & 1.000 & $\mathrm{Pd}$ \\
\hline Pd32 & 1.00000 .75000 & 0.83336 & 0.10989 & Biso & 1.000 & $\mathrm{Pd}$ \\
\hline $\operatorname{Pd} 33$ & 1.00000 .00003 & 0.16601 & 0.21321 & Biso & 1.000 & $\mathrm{Pd}$ \\
\hline $\operatorname{Pd} 34$ & 1.00000 .25071 & 0.16688 & 0.21404 & Biso & 1.000 & $\mathrm{Pd}$ \\
\hline Pd35 & 1.00000 .49822 & 0.16867 & 0.21633 & Biso & 1.000 & $\mathrm{Pd}$ \\
\hline $\operatorname{Pd} 36$ & 1.00000 .75006 & 0.16675 & 0.21386 & Biso & 1.000 & $\mathrm{Pd}$ \\
\hline $\operatorname{Pd} 37$ & 1.00000 .12537 & 0.41735 & 0.21413 & Biso & 1.000 & $\mathrm{Pd}$ \\
\hline $\operatorname{Pd} 38$ & 1.00000 .37510 & 0.41709 & 0.21380 & Biso & 1.000 & $\mathrm{Pd}$ \\
\hline Pd39 & 1.00000 .62620 & 0.41670 & 0.21357 & Biso & 1.000 & $\mathrm{Pd}$ \\
\hline $\operatorname{Pd} 40$ & 1.00000 .87581 & 0.41679 & 0.21382 & Biso & 1.000 & $\mathrm{Pd}$ \\
\hline Pd41 & 1.00000 .00053 & 0.66720 & 0.21416 & Biso & 1.000 & $\mathrm{Pd}$ \\
\hline $\operatorname{Pd} 42$ & 1.00000 .25023 & 0.66448 & 0.21640 & Biso & 1.000 & $\mathrm{Pd}$ \\
\hline $\operatorname{Pd} 43$ & 1.00000 .50048 & 0.66789 & 0.21354 & Biso & 1.000 & $\mathrm{Pd}$ \\
\hline Pd44 & 1.00000 .75058 & 0.66721 & 0.21385 & Biso & 1.000 & $\mathrm{Pd}$ \\
\hline $\mathrm{Pd} 45$ & 1.00000 .12600 & 0.91598 & 0.21406 & Biso & 1.000 & $\mathrm{Pd}$ \\
\hline $\operatorname{Pd} 46$ & 1.00000 .37545 & 0.91697 & 0.21393 & Biso & 1.000 & $\mathrm{Pd}$ \\
\hline $\operatorname{Pd} 47$ & 1.00000 .62481 & 0.91810 & 0.21396 & Biso & 1.000 & $\mathrm{Pd}$ \\
\hline $\operatorname{Pd} 48$ & 1.00000 .87523 & 0.91676 & 0.21385 & Biso & 1.000 & $\mathrm{Pd}$ \\
\hline $\operatorname{Pd} 49$ & 1.00000 .00066 & 0.00030 & 0.31919 & Biso & 1.000 & $\mathrm{Pd}$ \\
\hline $\operatorname{Pd} 50$ & 1.00000 .25107 & 0.00122 & 0.31949 & Biso & 1.000 & $\mathrm{Pd}$ \\
\hline $\operatorname{Pd} 51$ & 1.00000 .50292 & 0.00085 & 0.31995 & Biso & 1.000 & $\mathrm{Pd}$ \\
\hline $\operatorname{Pd} 52$ & 1.00000 .75202 & 0.00026 & 0.31956 & Biso & 1.000 & $\mathrm{Pd}$ \\
\hline
\end{tabular}




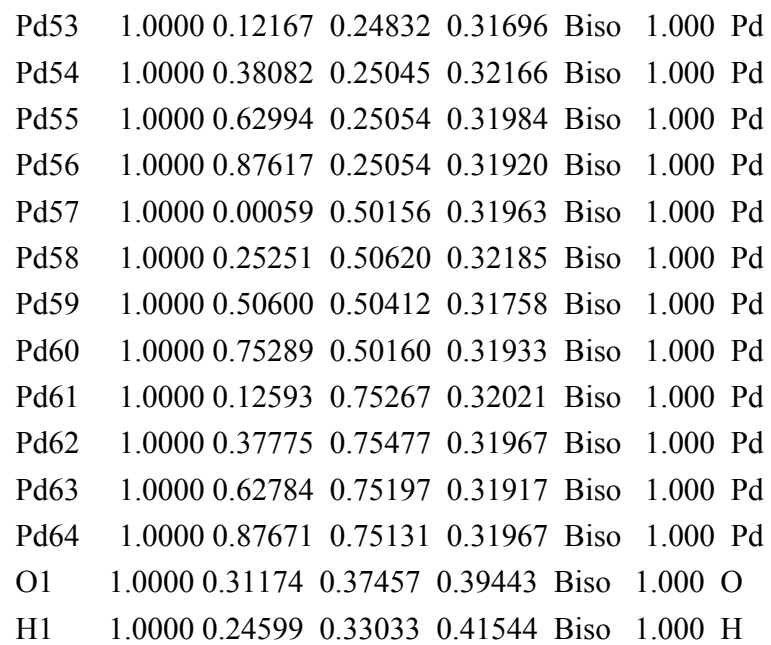




\begin{tabular}{|c|c|c|c|c|c|c|}
\hline 112 & 1.00000 .75000 & 0.49998 & 0.00440 & Biso & 1.000 & \\
\hline $\operatorname{Pd} 13$ & 00000.12500 & 0.74997 & 0.00440 & Biso & 1.000 & $\mathrm{Pd}$ \\
\hline d14 & 00000.37500 & 0.74998 & 0.00440 & Biso & 1.000 & $\mathrm{Pd}$ \\
\hline Pd15 & 1.00000 .62500 & 0.74998 & 0.00440 & Biso & 1.000 & $\mathrm{Pd}$ \\
\hline Pd16 & 00000.87500 & 0.74997 & 0.00440 & Biso & 1.000 & \\
\hline $\operatorname{Pd} 17$ & 500 & 0.083 & & Biso & 1.000 & \\
\hline d18 & 1.00000 .37500 & 0.08336 & 0.10989 & Biso & 1.000 & $\mathrm{Pd}$ \\
\hline Pd19 & 1.00000 .62500 & 0.08336 & .10989 & Biso & 1.000 & $\mathrm{Pd}$ \\
\hline $\mathrm{d} 20$ & 1.00000 .87500 & 0.08336 & 0.10988 & Biso & 1.000 & $\mathrm{Pd}$ \\
\hline $\operatorname{Pd} 21$ & 1.00000 .00000 & 0.33336 & 0.10989 & Biso & 1.000 & $\mathrm{Pd}$ \\
\hline $\operatorname{Pd} 22$ & 1.00000 .25000 & & & Biso & & $\mathrm{Pd}$ \\
\hline$d 23$ & 1.00000 .50000 & 0.333 & & Biso & 1.000 & $\mathrm{Pd}$ \\
\hline$d 24$ & 1.00 & 0. & & iso & 000 & $\mathrm{Pd}$ \\
\hline $\operatorname{Pd} 25$ & 1.00000 .12500 & 0.583 & & Biso & 1.000 & $\mathrm{Pd}$ \\
\hline $\operatorname{Pd} 26$ & 1.00000 .37501 & 0.58336 & 0.10989 & Biso & 1.000 & $\mathrm{Pd}$ \\
\hline $\operatorname{Pd} 27$ & 1.00000 .62499 & 0.583 & 0.10 & Biso & 1.000 & $\mathrm{Pd}$ \\
\hline $\mathrm{d} 28$ & 1.00000 .87500 & 0.58336 & & Biso & 1.000 & $\mathrm{Pd}$ \\
\hline$d 29$ & 1.00000 & 0.83 & 0.10988 & Biso & 1.000 & $\mathrm{Pd}$ \\
\hline $\mathrm{d} 30$ & 1.00000 & 0.83336 & 0.10989 & Biso & 1.000 & $\mathrm{Pd}$ \\
\hline $\operatorname{Pd} 31$ & 1.00000 .50000 & 0.83336 & 0.10989 & Biso & 1.000 & $\mathrm{Pd}$ \\
\hline $\operatorname{Pd} 32$ & 1.00000 .75000 & 0.83336 & 0.10989 & Biso & 1.000 & $\mathrm{Pd}$ \\
\hline $\mathrm{Pd} 33$ & 1.00000 .00017 & 0.165 & 0.21437 & Biso & 1.000 & $\mathrm{Pd}$ \\
\hline $\operatorname{Pd} 34$ & 1.00000 .25180 & 0.16721 & & Biso & 1.000 & $\mathrm{Pd}$ \\
\hline $\mathrm{d} 35$ & 1.00 & 0.16 & 0.21 & Biso & 1.000 & $\mathrm{Pd}$ \\
\hline 136 & 1.000 & 0.16 & 0.21646 & Biso & 1.000 & $\mathrm{Pd}$ \\
\hline 137 & 1.00000 & 0.41 & 0.21 & Biso & 1.000 & $\mathrm{Pd}$ \\
\hline $\operatorname{Pd} 38$ & 1.00000 .37475 & 0.41558 & 0.21408 & Biso & 1.000 & $\mathrm{Pd}$ \\
\hline Pd39 & 1.00000 .62536 & 0.41562 & 0.21391 & Biso & 1.000 & $\mathrm{Pd}$ \\
\hline $\mathrm{d} 40$ & 1.00000 .87555 & & & Biso & 1.000 & $\mathrm{Pd}$ \\
\hline Pd41 & $1.0000 \mathrm{C}$ & 0.66537 & 0.21381 & Biso & 1.000 & $\mathrm{Pd}$ \\
\hline Pd42 & 1.00 & 0.6 & 0.21 & Biso & 1.000 & $\mathrm{Pd}$ \\
\hline$d 43$ & 1.0000 & 0.66667 & 0.21 & Biso & 1.000 & $\mathrm{Pd}$ \\
\hline$d 44$ & 1.00000 .75061 & 0.66548 & 0.2136 & Biso & 1.000 & $\mathrm{Pd}$ \\
\hline $\operatorname{Pd} 45$ & 1.00000 .12508 & 0.91580 & 0.21391 & Biso & 1.000 & $\mathrm{Pd}$ \\
\hline Pd46 & 1.00000 .37450 & & & & 1.000 & $\mathrm{Pd}$ \\
\hline $\operatorname{Pd} 47$ & 1.00000 .62561 & 0.91615 & 0.21350 & Biso & 1.000 & $\mathrm{Pd}$ \\
\hline $\operatorname{Pd} 48$ & 1.00000 .87508 & 0.91594 & 0.21388 & Biso & 1.000 & $\mathrm{Pd}$ \\
\hline Pd49 & 1.00000 .00020 & 0.99647 & 0.31978 & Biso & 1.000 & $\mathrm{Pd}$ \\
\hline Pd50 & 1.00000 .24772 & 0.99425 & 0.31991 & Biso & 1.000 & $\mathrm{Pd}$ \\
\hline Pd51 & 1.00000 .50009 & 0.99206 & 0.31762 & Biso & 1.000 & $\mathrm{Pd}$ \\
\hline Pd52 & 1.00000 .75263 & 0.99436 & 0.31989 & Biso & 1.000 & $\mathrm{Pd}$ \\
\hline Pd53 & 1.00000 .12390 & 0.24693 & 0.32021 & Biso & 1.000 & $\mathrm{Pd}$ \\
\hline Pd54 & 1.00000 .37213 & 0.24357 & 0.32205 & Biso & 1.000 & $\mathrm{Pd}$ \\
\hline Pd55 & 1.00000 .62825 & 0.24332 & 0.3218 & Biso & 1.000 & $\mathrm{Pd}$ \\
\hline Pd56 & 1.00000 .87657 & 0.24693 & 0.32019 & Biso & 1.000 & $\mathrm{Pd}$ \\
\hline Pd57 & 1.00000 .00031 & 0.49742 & 0.31957 & Biso & 1.000 & $\mathrm{Pd}$ \\
\hline $\operatorname{Pd} 58$ & 1.00000 .24923 & 0.49766 & 0.31960 & Biso & 1.000 & $\mathrm{Pd}$ \\
\hline Pd59 & 1.00000 .50035 & 0.50258 & 0.31684 & Biso & 1.000 & $\mathrm{Pd}$ \\
\hline Pd60 & 1.00000 .7 & 0.49755 & 0.31901 & Biso & 1.000 & $\mathrm{Pd}$ \\
\hline Pd61 & 1.00000 .12445 & 0.74646 & 0.31953 & Biso & 1.000 & $\mathrm{Pd}$ \\
\hline Pd62 & 1.00000 .37422 & 0.74686 & 0.31907 & Biso & 1.000 & $\mathrm{Pd}$ \\
\hline Pd63 & 1.00000 .62618 & 0.74717 & 0.3184 & Biso & 1.000 & $\mathrm{Pd}$ \\
\hline Pd64 & 1.00000 .87600 & 0.74656 & 0.31939 & Biso & 1.000 & $\mathrm{Pd}$ \\
\hline
\end{tabular}




$\begin{array}{llllllll}\mathrm{O} 1 & 1.0000 & 0.50034 & 0.24719 & 0.39451 & \text { Biso } & 1.000 \mathrm{O} \\ \mathrm{H} 1 & 1.0000 & 0.50101 & 0.33435 & 0.41605 & \text { Biso } & 1.000 \mathrm{H} \\ \mathrm{C} 1 & 1.0000 & 0.58087 & 0.57614 & 0.48225 & \text { Biso } & 1.000 \mathrm{C} \\ \mathrm{C} 2 & 1.0000 & 0.70364 & 0.63777 & 0.49456 & \text { Biso } & 1.000 \mathrm{C} \\ \mathrm{C} 3 & 1.0000 & 0.47842 & 0.67201 & 0.49923 & \text { Biso } & 1.000 \mathrm{C} \\ \mathrm{H} 2 & 1.0000 & 0.57209 & 0.47898 & 0.50718 & \text { Biso } & 1.000 \mathrm{H} \\ \mathrm{H} 3 & 1.0000 & 0.57452 & 0.55101 & 0.43325 & \text { Biso } & 1.000 \mathrm{H} \\ \mathrm{H} 4 & 1.0000 & 0.77474 & 0.56733 & 0.48124 & \text { Biso } & 1.000 \mathrm{H} \\ \mathrm{H} 5 & 1.0000 & 0.39217 & 0.62528 & 0.48934 & \text { Biso } & 1.000 \mathrm{H} \\ \mathrm{H} 6 & 1.0000 & 0.71592 & 0.73324 & 0.46895 & \text { Biso } & 1.000 \mathrm{H} \\ \mathrm{H} 7 & 1.0000 & 0.71480 & 0.66142 & 0.54309 & \text { Biso } & 1.000 \mathrm{H} \\ \mathrm{H} 8 & 1.0000 & 0.48063 & 0.69736 & 0.54790 & \text { Biso } & 1.000 \mathrm{H} \\ \mathrm{H} 9 & 1.0000 & 0.48384 & 0.76828 & 0.47359 & \text { Biso } & 1.000 \mathrm{H}\end{array}$

\# Pd111(4x4).C1.1p E = -169.72; ZPVE $=\mathrm{eV}$; frequencies $=\mathrm{cm}^{\wedge}-1$

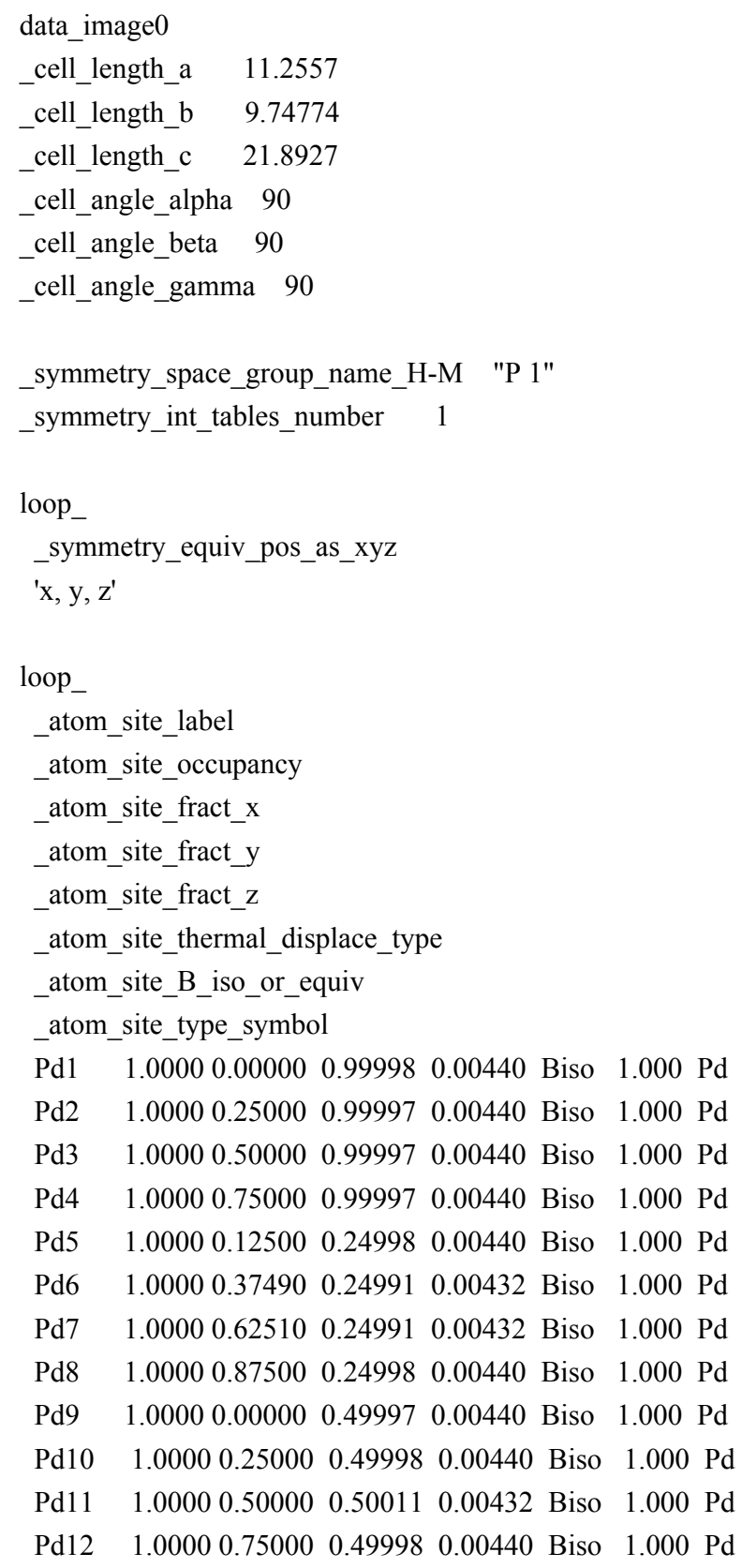




\begin{tabular}{|c|c|c|c|c|c|c|}
\hline $\operatorname{Pd} 13$ & 1.00000 .12500 & 0.74997 & 0.00440 & Biso & 1.000 & \\
\hline $\mathrm{d} 14$ & 1.00000 .37500 & 0.74998 & 0.00440 & Biso & 1.000 & \\
\hline d15 & .00000 .62500 & 0.74998 & 0.00440 & Biso & 1.000 & $\mathrm{Pd}$ \\
\hline 116 & 1.00000 .87500 & 0.74997 & 0.00440 & Biso & 1.000 & $\mathrm{Pd}$ \\
\hline Pd17 & 1.00000 .12500 & 0.08336 & 0.10988 & Biso & 1.000 & $\mathrm{P}$ \\
\hline Pd18 & 500 & & & Biso & 1.000 & \\
\hline Pd19 & 500 & & 10989 & Biso & 1.000 & $\mathrm{Pd}$ \\
\hline $\mathrm{d} 20$ & 1.00000 .87500 & 0.083 & 10988 & Biso & 1.000 & $\mathrm{Pd}$ \\
\hline $\operatorname{Pd} 21$ & 1.00000 .00000 & 0.33336 & 10989 & Biso & 1.000 & $\mathrm{Pd}$ \\
\hline $\mathrm{d} 22$ & 1.00000 .25000 & 0.33336 & 0.10989 & Biso & 1.000 & $\mathrm{Pd}$ \\
\hline $\mathrm{Pd} 23$ & 1.00000 .50000 & 0.33333 & & Biso & 1.000 & $\mathrm{Pd}$ \\
\hline$d 24$ & 1.00000 .75000 & & & Biso & 1.000 & $\mathrm{Pd}$ \\
\hline$d 25$ & 1.00000 .12500 & 0.58 & 988 & Biso & 1.000 & $\mathrm{Pd}$ \\
\hline $\operatorname{Pd} 26$ & $1.00000 .3^{\prime}$ & 0.58336 & & Biso & 1.000 & $\mathrm{Pd}$ \\
\hline $\operatorname{Pd} 27$ & 1.00000 .62499 & 0.58336 & 0.10989 & Biso & 1.000 & $\mathrm{Pd}$ \\
\hline $\operatorname{Pd} 28$ & 1.00000 .87500 & 0.58336 & 0.10988 & Biso & 1.000 & $\mathrm{Pd}$ \\
\hline $\operatorname{Pd} 29$ & 1.00000 .00000 & 0.83336 & 0.10988 & Biso & 1.000 & $\mathrm{Pd}$ \\
\hline $\mathrm{d} 30$ & 1.00000 .25000 & 0.833 & & Biso & 1.000 & $\mathrm{Pd}$ \\
\hline 131 & 1.0000 & 0.8 & 0.10 & Biso & 1.000 & $\mathrm{Pd}$ \\
\hline $\mathrm{d} 32$ & 1.00000 & 0.83336 & 0.10989 & Biso & 1.000 & $\mathrm{Pd}$ \\
\hline $\operatorname{Pd} 33$ & 1.00000 .99973 & 0.16702 & 0.21389 & Biso & 1.000 & $\mathrm{Pd}$ \\
\hline $\operatorname{Pd} 34$ & 1.00000 .25032 & 0.16611 & 0.21 & Biso & 1.000 & $\mathrm{Pd}$ \\
\hline Pd35 & 1.00000 .50015 & 0.16649 & & Biso & 1.000 & $\mathrm{Pd}$ \\
\hline $\operatorname{Pd} 36$ & 1.00000 .74961 & 0.16 & & Biso & 1.000 & $\mathrm{Pd}$ \\
\hline $\mathrm{d} 37$ & 1.00 & & & Biso & 1.000 & $\mathrm{Pd}$ \\
\hline $\mathrm{d} 38$ & 1.0000 & 0.4 & 0.2 & Biso & 1.000 & $\mathrm{Pd}$ \\
\hline $\mathrm{d} 39$ & 1.00000 .62355 & 0.41750 & 0.21533 & Biso & 1.000 & $\mathrm{Pd}$ \\
\hline $\operatorname{Pd} 40$ & 1.00000 .87462 & 0.41660 & 0.21387 & Biso & 1.000 & $\mathrm{Pd}$ \\
\hline Pd41 & 1.00000 .00003 & 0.66675 & 0.21386 & Biso & 1.000 & $\mathrm{Pd}$ \\
\hline $\operatorname{Pd} 42$ & 1.00000 .24978 & 0.66658 & 0.21376 & Biso & 1.000 & $\mathrm{Pd}$ \\
\hline $\operatorname{Pd} 43$ & 1.00000 .49993 & 0.66527 & 0.21529 & Biso & 1.000 & $\mathrm{Pd}$ \\
\hline $\operatorname{Pd} 44$ & 1.000 & 0.666 & 0.21380 & Biso & 1.000 & $\mathrm{Pd}$ \\
\hline$d 45$ & 1.0000 & 0.91 & 0.21449 & Biso & 1.000 & $\mathrm{Pd}$ \\
\hline d46 & 1.00000 .37445 & 0.91657 & 0.21418 & Biso & 1.000 & $\mathrm{Pd}$ \\
\hline $\operatorname{Pd} 47$ & 1.00000 .62499 & 0.91593 & 0.21372 & Biso & 1.000 & $\mathrm{Pd}$ \\
\hline Pd48 & 1.00000 .87511 & 0.91672 & & Biso & 1.000 & $\mathrm{Pd}$ \\
\hline Pd49 & 1.00000 .99878 & 0.00026 & 0.31932 & Biso & 1.000 & $\mathrm{Pd}$ \\
\hline Pd50 & 1.00000 .24982 & 0.99953 & 0.32124 & Biso & 1.000 & $\mathrm{Pd}$ \\
\hline Pd51 & 1.00000 .50102 & 0.00010 & 0.3189 & Biso & 1.000 & $\mathrm{Pd}$ \\
\hline Pd52 & 1.00000 .74980 & 0.00006 & 0.31945 & Biso & 1.000 & $\mathrm{Pd}$ \\
\hline Pd53 & 1.00000 .12448 & 0.25061 & 0.31918 & Biso & 1.000 & $\mathrm{Pd}$ \\
\hline Pd54 & 1.00000 .3 & & & Biso & 1.000 & $\mathrm{Pd}$ \\
\hline Pd55 & 1.00000 .62509 & 0.24975 & 0.31909 & Biso & 1.000 & $\mathrm{Pd}$ \\
\hline Pd56 & 1.00000 .87 & 0.250 & 0.31956 & Biso & 1.000 & $\mathrm{Pd}$ \\
\hline Pd57 & 1.00000 .99983 & 0.49988 & 0.31938 & Biso & 1.000 & $\mathrm{Pd}$ \\
\hline Pd58 & 1.00000 .24953 & 0.49957 & 0.31905 & Biso & 1.000 & $\mathrm{Pd}$ \\
\hline Pd59 & 1.00000 .50017 & 0.50004 & 0.32858 & Biso & 1.000 & $\mathrm{Pd}$ \\
\hline Pd60 & 1.00000 .75036 & 0.50016 & 0.31897 & Biso & 1.000 & $\mathrm{Pd}$ \\
\hline Pd61 & 1.00000 .12431 & 0.74915 & 0.31921 & Biso & 1.000 & $\mathrm{Pd}$ \\
\hline Pd62 & 1.00000 .37531 & 0.74953 & 0.31878 & Biso & 1.000 & $\mathrm{Pd}$ \\
\hline Pd63 & 1.00000 .62524 & 0.75029 & 0.31932 & Biso & 1.000 & $\mathrm{Pd}$ \\
\hline Pd64 & 1.00000 .87478 & 0.74994 & 0.3194 & Biso & 1.000 & $\mathrm{Pd}$ \\
\hline & 1.00000 .25870 & 00527 & 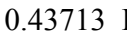 & Biso & $1.000 \mathrm{C}$ & \\
\hline
\end{tabular}




$\begin{array}{llllllll}\mathrm{H} 1 & 1.0000 & 0.21151 & 0.08606 & 0.44593 & \text { Biso } & 1.000 \mathrm{H} \\ \mathrm{C} 1 & 1.0000 & 0.60033 & 0.44820 & 0.45536 & \text { Biso } & 1.000 \mathrm{C} \\ \mathrm{C} 2 & 1.0000 & 0.58669 & 0.45935 & 0.52531 & \text { Biso } & 1.000 \mathrm{C} \\ \mathrm{C} 3 & 1.0000 & 0.49259 & 0.50726 & 0.42317 & \text { Biso } & 1.000 \mathrm{C} \\ \mathrm{H} 2 & 1.0000 & 0.61282 & 0.34018 & 0.44290 & \text { Biso } & 1.000 \mathrm{H} \\ \mathrm{H} 3 & 1.0000 & 0.68115 & 0.50281 & 0.44146 & \text { Biso } & 1.000 \mathrm{H} \\ \mathrm{H} 4 & 1.0000 & 0.66481 & 0.41674 & 0.54809 & \text { Biso } & 1.000 \mathrm{H} \\ \mathrm{H} 5 & 1.0000 & 0.34039 & 0.03383 & 0.44302 & \text { Biso } & 1.000 \mathrm{H} \\ \mathrm{H} 6 & 1.0000 & 0.57716 & 0.56634 & 0.53947 & \text { Biso } & 1.000 \mathrm{H} \\ \text { H7 } & 1.0000 & 0.50848 & 0.40310 & 0.54115 & \text { Biso } & 1.000 \mathrm{H} \\ \text { H8 } & 1.0000 & 0.41080 & 0.45127 & 0.43371 & \text { Biso } & 1.000 \mathrm{H} \\ \text { H9 } & 1.0000 & 0.48019 & 0.61656 & 0.43277 & \text { Biso } & 1.000 \mathrm{H}\end{array}$

\# Pd211(6x6).A1.P E= -292.46; ZPVE $=\mathrm{eV}$; frequencies $=\mathrm{cm}^{\wedge}-1$

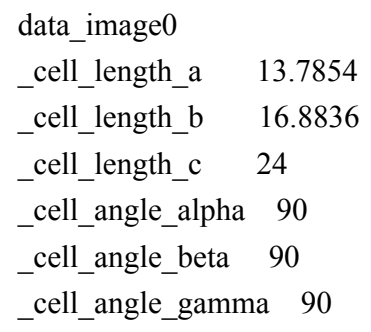




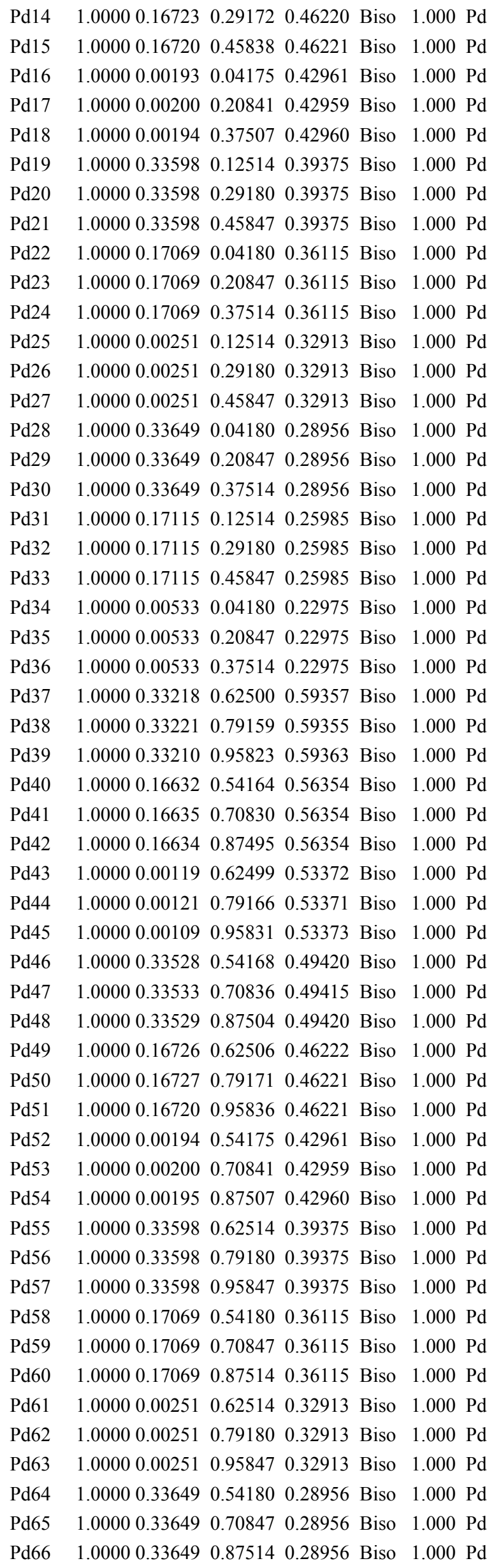




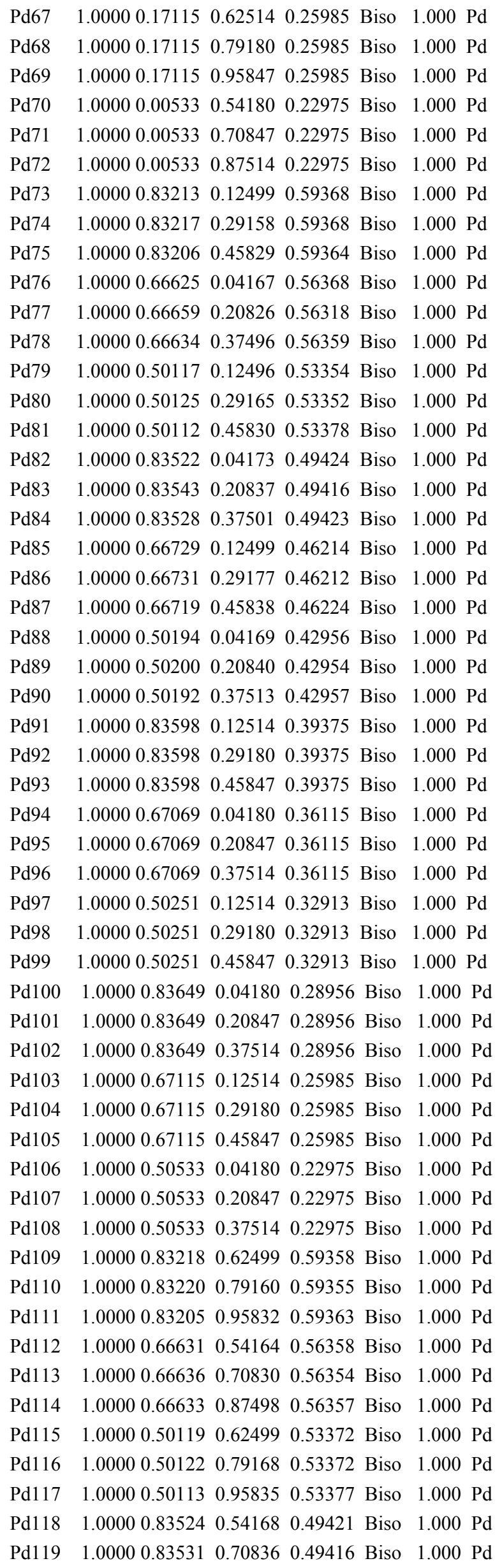




\begin{tabular}{|c|c|c|c|}
\hline d120 & $\begin{array}{lll}1.0000 & 0.83527 & 0.87505\end{array}$ & 50.49420 Biso & \\
\hline $\operatorname{Pd} 121$ & 1.00000 .667250 .62505 & 50.46222 Biso & $1.000 \mathrm{Pd}$ \\
\hline 1122 & $\begin{array}{lll}1.0000 & 0.66727 & 0.79172\end{array}$ & 20.46222 Biso & 1.000 \\
\hline 1123 & $\begin{array}{lll}1.0000 & 0.66719 & 0.95839\end{array}$ & 90.46225 Biso & 000 \\
\hline & $\begin{array}{lll}1.0000 & 0.50195 & 0.54176\end{array}$ & 61 Biso & 1.000 \\
\hline d125 & $\begin{array}{lll}1.0000 & 0.50201 & 0.70841\end{array}$ & 10.42959 Biso & 1.000 \\
\hline 1126 & 1.00000 & 60.42960 Biso & 1.000 \\
\hline d127 & 5980.6 & 39375 Biso & 000 \\
\hline d128 & $\begin{array}{lll}1.0000 & 0.83598 & 0.79180\end{array}$ & 00.39375 Biso & 1.000 \\
\hline d129 & $\begin{array}{lll}1.0000 & 0.83598 & 0.95847\end{array}$ & 70.39375 Biso & 1.000 \\
\hline d130 & 70690.54180 & 15 Biso & $1.000 \mathrm{I}$ \\
\hline 4151 & $\begin{array}{ll}069 & 0.70847\end{array}$ & 15 Biso & $1.000 \mathrm{~F}$ \\
\hline 1132 & $\begin{array}{lll}1.0000 & 0.67069 & 0.87514\end{array}$ & 40.36115 Biso & $1.000 \mathrm{~F}$ \\
\hline d133 & $\begin{array}{llll}1.0000 & 0.50251 & 0.62514\end{array}$ & 40.32913 Biso & $1.000 \mathrm{I}$ \\
\hline Pd134 & $\begin{array}{lll}1.0000 & 0.50251 & 0.79180\end{array}$ & 00.32913 Biso & $1.000 \mathrm{I}$ \\
\hline d135 & $\begin{array}{lll}1.0000 & 0.50251 & 0.95847\end{array}$ & 70.3291 & 1.00 \\
\hline $\operatorname{Pd} 136$ & 1.00000 .836490 .54180 & 00.28956 Biso & $1.000 \mathrm{Pd}$ \\
\hline d137 & $\begin{array}{lll}1.0000 & 0.83649 & 0.70847\end{array}$ & 56 Biso & $1.000 \mathrm{P}$ \\
\hline 1138 & $83649 \quad 0.87514$ & 40.28956 Biso & $1.000 \mathrm{P}$ \\
\hline 1139 & 1150.62514 & 85 Biso & $1.000 \mathrm{I}$ \\
\hline $\operatorname{Pd} 140$ & 1.00000 .671150 .79180 & 00.25985 Biso & $1.000 \mathrm{Pd}$ \\
\hline d141 & $\begin{array}{lll}1.0000 & 0.67115 & 0.95847\end{array}$ & 70.25985 Biso & 1.000 \\
\hline Pd142 & 1.00000 .505330 .54180 & 00.22975 Biso & $1.000 \mathrm{I}$ \\
\hline $\operatorname{Pd} 143$ & $\begin{array}{lll}1.0000 & 0.50533 & 0.70847\end{array}$ & 7 0.22975 Biso & $1.000 \mathrm{~F}$ \\
\hline Pd144 & $\begin{array}{lll}1.0000 & 0.50533 & 0.875\end{array}$ & 40.22975 Biso & $1.000 \mathrm{P}$ \\
\hline $\mathrm{C} 1$ & $\begin{array}{lll}1.0000 & 0.51250 & 0.22960\end{array}$ & 70869 Biso & $1.000 \mathrm{C}$ \\
\hline $\mathrm{C} 2$ & $1.0000 \quad 0.60436 \quad 0.27453$ & 0.72557 Biso & $1.000 \mathrm{C}$ \\
\hline $\mathrm{C} 3$ & $\begin{array}{llll}1.0000 & 0.51956 & 0.14027\end{array}$ & 0.71886 Biso & $1.000 \mathrm{C}$ \\
\hline H1 & $\begin{array}{lll}1.0000 & 0.49793 & 0.24031\end{array}$ & 0.66432 Biso & $1.000 \mathrm{H}$ \\
\hline $\mathrm{H} 2$ & $\begin{array}{lll}1.0000 & 0.45013 & 0.25360\end{array}$ & 0.73154 Biso & $1.000 \mathrm{H}$ \\
\hline H3 & $\begin{array}{lll}1.0000 & 0.59801 & 0.33784\end{array}$ & 0.71646 Biso & $1.000 \mathrm{H}$ \\
\hline H4 & $\begin{array}{lll}1.0000 & 0.45313 & 0.10970\end{array}$ & 0.70604 Biso & $1.000 \mathrm{H}$ \\
\hline H5 & $\begin{array}{lll}1.0000 & 0.61887 & 0.26802\end{array}$ & 0.77018 Biso & $1.000 \mathrm{H}$ \\
\hline H6 & $1.0000 \quad 0.66770 \quad 0.25204$ & 0.70297 Biso & $1.000 \mathrm{H}$ \\
\hline H7 & $\begin{array}{llll}1.0000 & 0.57977 & 0.11437\end{array}$ & 0.69527 Biso & $1.000 \mathrm{H}$ \\
\hline H8 & $\begin{array}{lll}1.0000 & 0.53155 & 0.12734\end{array}$ & 0.76302 Biso & $1.000 \mathrm{H}$ \\
\hline
\end{tabular}

\# Pd211(6x6).A1.1p E= ; ZPVE $=-291.99 \mathrm{eV}$; frequencies $=\mathrm{cm}^{\wedge}-1$

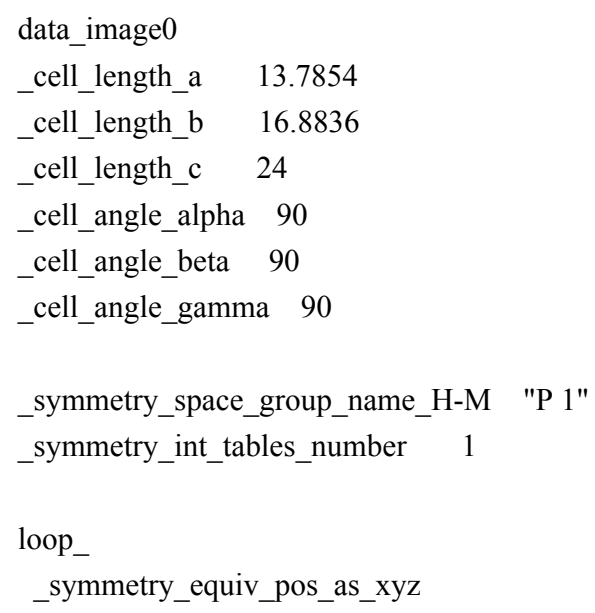




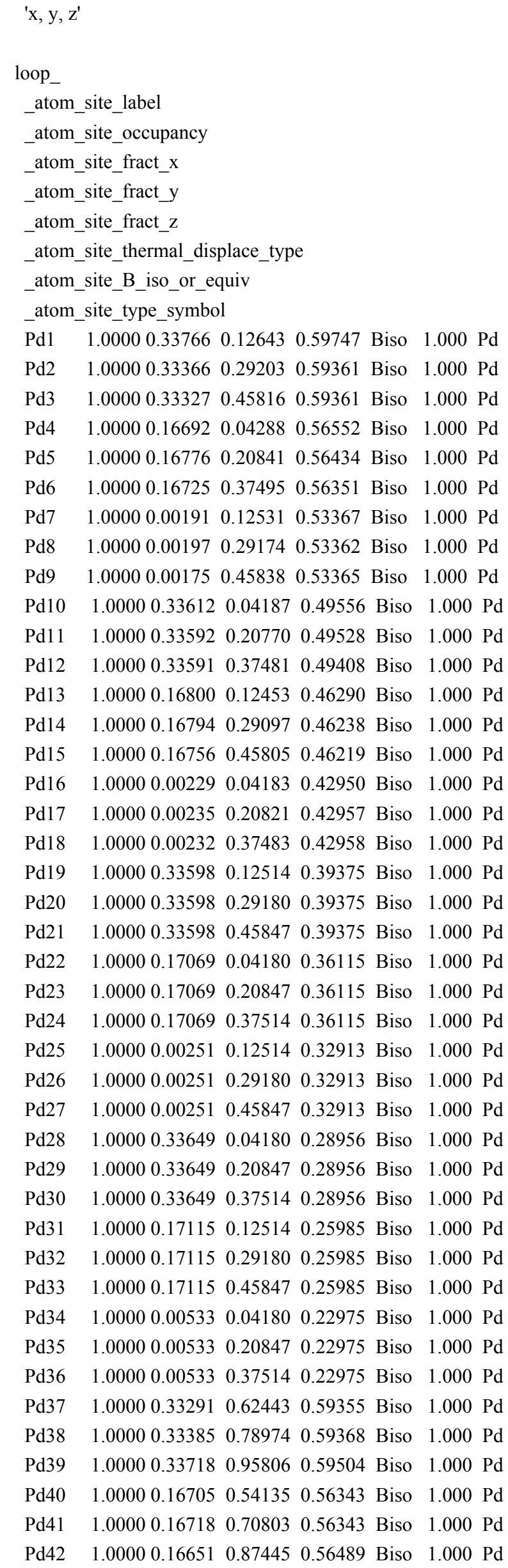


Pd43 $1.00000 .001760 .62496 \quad 0.53369$ Biso 1.000 Pd

$\begin{array}{llllllll}\mathrm{Pd} 44 & 1.0000 & 0.00150 & 0.79151 & 0.53360 & \text { Biso } 1.000 \mathrm{Pd}\end{array}$

Pd45 1.00000 .001040 .958450 .53331 Biso 1.000 Pd

$\begin{array}{llllllll}\text { Pd46 } & 1.0000 & 0.33557 & 0.54147 & 0.49413 & \text { Biso } 1.000 & \text { Pd }\end{array}$

$\begin{array}{llllllll}\mathrm{Pd} 47 & 1.0000 & 0.33580 & 0.70818 & 0.49402 & \text { Biso } 1.000 & \mathrm{Pd}\end{array}$

$\begin{array}{llllllll}\mathrm{Pd} 48 & 1.0000 & 0.33609 & 0.87511 & 0.49484 & \text { Biso } 1.000 \mathrm{Pd}\end{array}$

$\begin{array}{llllllll}\mathrm{Pd} 49 & 1.0000 & 0.16749 & 0.62521 & 0.46212 & \text { Biso } 1.000 \mathrm{Pd}\end{array}$

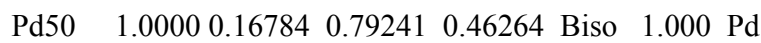

$\begin{array}{llllllll}\mathrm{Pd} 51 & 1.0000 & 0.16781 & 0.95876 & 0.46279 & \text { Biso } 1.000 \mathrm{Pd}\end{array}$

$\begin{array}{lllllllll}\text { Pd52 } & 1.0000 & 0.00218 & 0.54174 & 0.42957 & \text { Biso } 1.000 & \text { Pd }\end{array}$

$\begin{array}{llllllll}\mathrm{Pd} 53 & 1.0000 & 0.00231 & 0.70855 & 0.42961 & \text { Biso } 1.000 \mathrm{Pd}\end{array}$

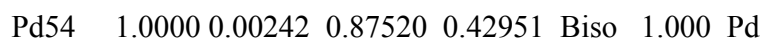

$\begin{array}{llllllll}\text { Pd55 } & 1.0000 & 0.33598 & 0.62514 & 0.39375 & \text { Biso } 1.000 & \text { Pd }\end{array}$

$\begin{array}{lllllll}\text { Pd56 } & 1.0000 & 0.33598 & 0.79180 & 0.39375 & \text { Biso } 1.000 & P d\end{array}$

$\begin{array}{llllllll}\mathrm{Pd} 57 & 1.0000 & 0.33598 & 0.95847 & 0.39375 & \text { Biso } & 1.000 & \mathrm{Pd}\end{array}$

$\begin{array}{llllllll}\text { Pd58 } & 1.0000 & 0.17069 & 0.54180 & 0.36115 & \text { Biso } 1.000 & \mathrm{Pd}\end{array}$

$\begin{array}{llllllll}\text { Pd59 } 1.0000 & 0.17069 & 0.70847 & 0.36115 & \text { Biso } 1.000 & \mathrm{Pd}\end{array}$

$\begin{array}{lllllll}\text { Pd60 } & 1.0000 & 0.17069 & 0.87514 & 0.36115 & \text { Biso } 1.000 & P d\end{array}$

$\begin{array}{lllllllll}\text { Pd61 } & 1.0000 & 0.00251 & 0.62514 & 0.32913 & \text { Biso } 1.000 & \text { Pd }\end{array}$

$\begin{array}{lllllllll}\text { Pd62 } & 1.0000 & 0.00251 & 0.79180 & 0.32913 & \text { Biso } 1.000 & \text { Pd }\end{array}$

$\begin{array}{llllllll}\text { Pd63 } & 1.0000 & 0.00251 & 0.95847 & 0.32913 & \text { Biso } 1.000 & \text { Pd }\end{array}$

$\begin{array}{llllllll}\text { Pd64 } 1.0000 & 0.33649 & 0.54180 & 0.28956 & \text { Biso } 1.000 & \mathrm{Pd}\end{array}$

$\begin{array}{lllllllll}\text { Pd65 } & 1.0000 & 0.33649 & 0.70847 & 0.28956 & \text { Biso } 1.000 & \text { Pd }\end{array}$

$\begin{array}{lllllllll}\text { Pd66 } & 1.0000 & 0.33649 & 0.87514 & 0.28956 & \text { Biso } 1.000 & \mathrm{Pd}\end{array}$

$\begin{array}{lllllllll}\text { Pd67 } & 1.0000 & 0.17115 & 0.62514 & 0.25985 & \text { Biso } 1.000 & \text { Pd }\end{array}$

$\begin{array}{llllllll}\text { Pd68 } & 1.0000 & 0.17115 & 0.79180 & 0.25985 & \text { Biso } 1.000 & \mathrm{Pd}\end{array}$

$\begin{array}{llllllll}\text { Pd69 } & 1.0000 & 0.17115 & 0.95847 & 0.25985 & \text { Biso } 1.000 & P d\end{array}$

$\begin{array}{llllllll}\mathrm{Pd} 70 & 1.0000 & 0.00533 & 0.54180 & 0.22975 & \text { Biso } 1.000 \mathrm{Pd}\end{array}$

$\begin{array}{llllllll}\mathrm{Pd} 71 & 1.0000 & 0.00533 & 0.70847 & 0.22975 & \text { Biso } 1.000 \mathrm{Pd}\end{array}$

$\begin{array}{llllllll}\mathrm{Pd} 72 & 1.0000 & 0.00533 & 0.87514 & 0.22975 & \text { Biso } 1.000 \mathrm{Pd}\end{array}$

$\begin{array}{llllllll}\mathrm{Pd} 73 & 1.0000 & 0.83293 & 0.12509 & 0.59361 & \text { Biso } 1.000 \mathrm{Pd}\end{array}$

$\begin{array}{lllllllll}\text { Pd74 } & 1.0000 & 0.83323 & 0.29170 & 0.59367 & \text { Biso } 1.000 & \text { Pd }\end{array}$

$\begin{array}{llllllll}\operatorname{Pd} 75 & 1.0000 & 0.83310 & 0.45843 & 0.59367 & \text { Biso } 1.000 & \mathrm{Pd}\end{array}$

$\begin{array}{llllllll}\mathrm{Pd} 76 & 1.0000 & 0.66709 & 0.04175 & 0.56352 & \text { Biso } 1.000 \mathrm{Pd}\end{array}$

$\begin{array}{llllllll}\mathrm{Pd} 77 & 1.0000 & 0.66734 & 0.20850 & 0.56354 & \text { Biso } 1.000 \mathrm{Pd}\end{array}$

$\begin{array}{llllllll}\mathrm{Pd} 78 & 1.0000 & 0.66744 & 0.37511 & 0.56363 & \text { Biso } 1.000 \mathrm{Pd}\end{array}$

$\begin{array}{llllllll}\operatorname{Pd} 79 & 1.0000 & 0.50213 & 0.12508 & 0.53386 & \text { Biso } 1.000 & \mathrm{Pd}\end{array}$

$\begin{array}{llllllll}\mathrm{Pd} 80 & 1.0000 & 0.50254 & 0.29212 & 0.53352 & \text { Biso } & 1.000 & \mathrm{Pd}\end{array}$

$\begin{array}{llllllll}\mathrm{Pd} 81 & 1.0000 & 0.50193 & 0.45840 & 0.53376 & \text { Biso } 1.000 \mathrm{Pd}\end{array}$

$\begin{array}{llllllll}\mathrm{Pd} 82 & 1.0000 & 0.83588 & 0.04199 & 0.49418 & \text { Biso } & 1.000 & \mathrm{Pd}\end{array}$

$\begin{array}{llllllll}\mathrm{Pd} 83 & 1.0000 & 0.83566 & 0.20862 & 0.49413 & \text { Biso } & 1.000 & \mathrm{Pd}\end{array}$

$\begin{array}{lllllllll}\mathrm{Pd} 84 & 1.0000 & 0.83581 & 0.37511 & 0.49423 & \text { Biso } & 1.000 & \mathrm{Pd}\end{array}$

$\begin{array}{lllllllll}\mathrm{Pd} 85 & 1.0000 & 0.66705 & 0.12510 & 0.46229 & \text { Biso } & 1.000 & \mathrm{Pd}\end{array}$

$\begin{array}{lllllllll}\mathrm{Pd} 86 & 1.0000 & 0.66761 & 0.29176 & 0.46213 & \text { Biso } & 1.000 & \mathrm{Pd}\end{array}$

$\begin{array}{lllllllll}\mathrm{Pd} 87 & 1.0000 & 0.66758 & 0.45840 & 0.46226 & \text { Biso } & 1.000 & \mathrm{Pd}\end{array}$

$\begin{array}{lllllllll}\mathrm{Pd} 88 & 1.0000 & 0.50181 & 0.04175 & 0.42948 & \text { Biso } & 1.000 & \mathrm{Pd}\end{array}$

$\begin{array}{llllllll}\mathrm{Pd} 89 & 1.0000 & 0.50162 & 0.20855 & 0.42960 & \text { Biso } 1.000 \mathrm{Pd}\end{array}$

$\begin{array}{llllllll}\mathrm{Pd} 90 & 1.0000 & 0.50220 & 0.37518 & 0.42951 & \text { Biso } 1.000 \mathrm{Pd}\end{array}$

$\begin{array}{llllllll}\text { Pd91 } & 1.0000 & 0.83598 & 0.12514 & 0.39375 & \text { Biso } 1.000 & \mathrm{Pd}\end{array}$

$\begin{array}{llllllll}\text { Pd92 } & 1.0000 & 0.83598 & 0.29180 & 0.39375 & \text { Biso } 1.000 & \mathrm{Pd}\end{array}$

$\begin{array}{lllllll}\mathrm{Pd} 93 & 1.0000 & 0.83598 & 0.45847 & 0.39375 & \text { Biso } 1.000 \mathrm{Pd}\end{array}$

$\begin{array}{llllllll}\mathrm{Pd} 94 & 1.0000 & 0.67069 & 0.04180 & 0.36115 & \text { Biso } 1.000 \mathrm{Pd}\end{array}$

Pd95 1.00000 .670690 .208470 .36115 Biso $1.000 \mathrm{Pd}$ 


\begin{tabular}{|c|c|c|c|c|}
\hline & & & 15 Biso & \\
\hline & 1.00000 .50251 & & 0.32913 Biso & $.000 \mathrm{P}$ \\
\hline & & & $13 \mathrm{E}$ & \\
\hline & & & $13 \mathrm{E}$ & \\
\hline & & & 56 Biso & 000 \\
\hline & 19 & & & \\
\hline & & & & \\
\hline 1103 & & & & \\
\hline 1104 & 5 & & 85 Biso & 00 \\
\hline & & & 85 Biso & \\
\hline & & & 75 Biso & \\
\hline & & & & \\
\hline & & & & \\
\hline 109 & 287 & & 63 Biso & \\
\hline 1110 & & & & \\
\hline 1111 & 100 & & 1 Biso & 00 \\
\hline 1112 & 1.00 & & 67 Biso & $1.000 \mathrm{I}$ \\
\hline & & & & \\
\hline & & & 51 Biso & \\
\hline & & & 78 Biso & \\
\hline 116 & 210 & & $68 \mathrm{~B}$ & $00 \mathrm{I}$ \\
\hline 117 & 1.00000 & 30.9 & 66 Biso & 00 \\
\hline 1118 & 1.0000 & & 22 Biso & 1 \\
\hline & & & 3 Biso & \\
\hline & & & 14 Biso & \\
\hline 1121 & & & 28 Biso & 1. \\
\hline & & & $25 \mathrm{~B}$ & 1 \\
\hline & & & 78 Biso & $00 \mathrm{I}$ \\
\hline 1124 & 100000 & 0.54175 & 57 Biso & $1.000 \mathrm{I}$ \\
\hline & 1.00000 .50215 & & 52 Biso & 1000 \\
\hline & & & 48 Biso & \\
\hline & & & 75 Biso & $00 \mathrm{P}$ \\
\hline & & & $\mathrm{Bi}$ & \\
\hline 129 & & & 75 Biso & $1.000 \mathrm{P}$ \\
\hline & & & Biso & \\
\hline & & & 15 Biso & $1.000 \mathrm{P}$ \\
\hline & & & Biso & \\
\hline 133 & 1.00 & 0.62 & 13 Biso & 1.0 \\
\hline & & & Biso & 1.0 \\
\hline & 1.0 & & 3 Biso & $1.000 \mathrm{~F}$ \\
\hline & 649 & 0.54180 & 0.28956 Biso & 1.000 \\
\hline & & & 956 Biso & $1.000 \mathrm{P}$ \\
\hline & & & 56 Biso & \\
\hline & 1.00000 & & 985 Biso & $1.000 \mathrm{P}$ \\
\hline & & & 85 Biso & $00 \mathrm{I}$ \\
\hline 114 & 1.000 & & 85 Biso & $1.000 \mathrm{I}$ \\
\hline 1142 & 1.00 & 0.54180 & 0.22975 Biso & 1.000 \\
\hline 1142 & 1.0000 & 0.70847 & 7 0.22975 Biso & $1.000 \mathrm{~F}$ \\
\hline & 10000050522 & & 0.22975 Biso & $1.000 \mathrm{Pd}$ \\
\hline & 226 & & 0.69192 Biso & $1.000 \mathrm{C}$ \\
\hline & 1.00000 & 0.148960 & 0.75217 Biso & $1.000 \mathrm{C}$ \\
\hline & 1.00000 .385090 & 0.112590 & 0.67812 Biso & $1.000 \mathrm{C}$ \\
\hline & 50502000 & 0.15081 & 0.66325 Biso & $1.000 \mathrm{H}$ \\
\hline
\end{tabular}




$\begin{array}{llllllll}\text { H2 } & 1.0000 & 0.45466 & 0.22659 & 0.68693 & \text { Biso } & 1.000 \mathrm{H} \\ \mathrm{H} 3 & 1.0000 & 0.56963 & 0.18675 & 0.76139 & \text { Biso } & 1.000 \mathrm{H} \\ \mathrm{H} 4 & 1.0000 & 0.20697 & 0.95852 & 0.60644 & \text { Biso } & 1.000 \mathrm{H} \\ \mathrm{H} 5 & 1.0000 & 0.45005 & 0.16216 & 0.78239 & \text { Biso } & 1.000 \mathrm{H} \\ \mathrm{H} 6 & 1.0000 & 0.52927 & 0.08721 & 0.75786 & \text { Biso } & 1.000 \mathrm{H} \\ \mathrm{H} 7 & 1.0000 & 0.40102 & 0.04916 & 0.68198 & \text { Biso } & 1.000 \mathrm{H} \\ \mathrm{H} 8 & 1.0000 & 0.31984 & 0.12754 & 0.70200 & \text { Biso } & 1.000 \mathrm{H}\end{array}$

\# Pd211(6x6).Ads.OH E = -246.67; ZPVE $=\mathrm{eV}$; frequencies $=\mathrm{cm}^{\wedge}-1$

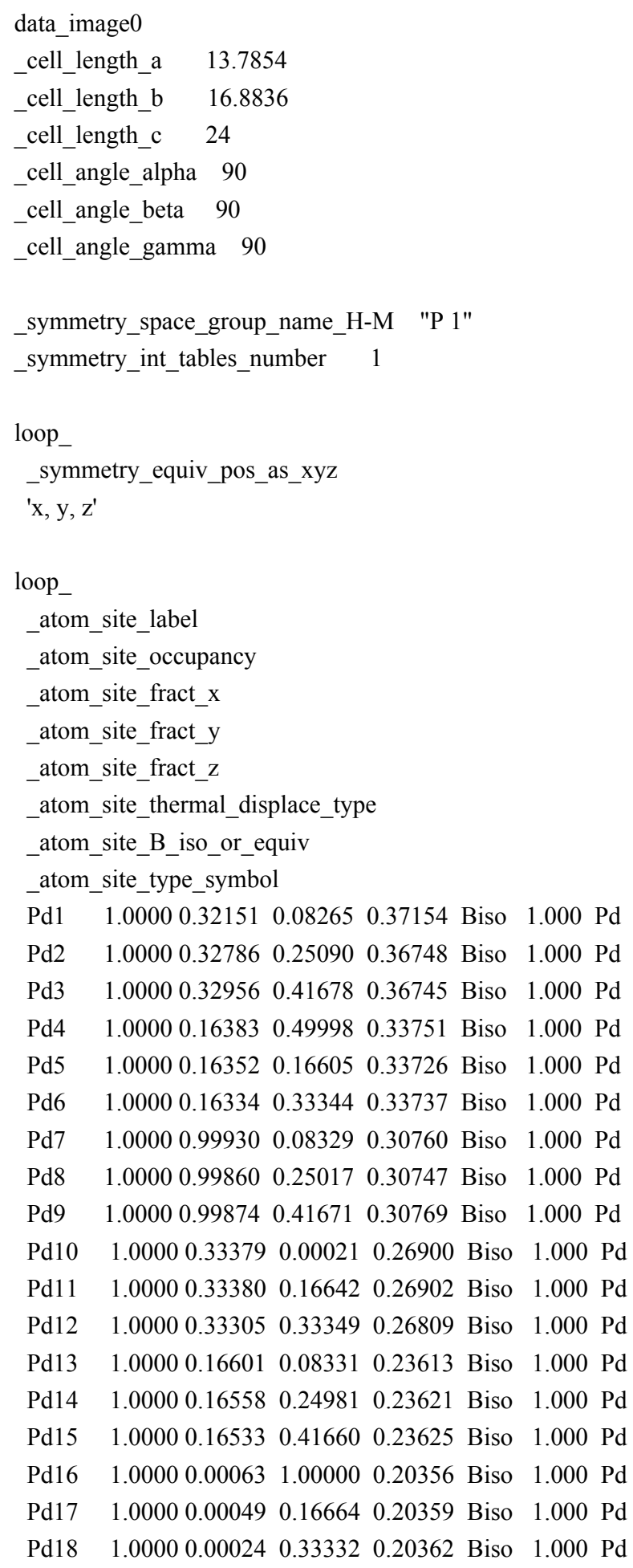




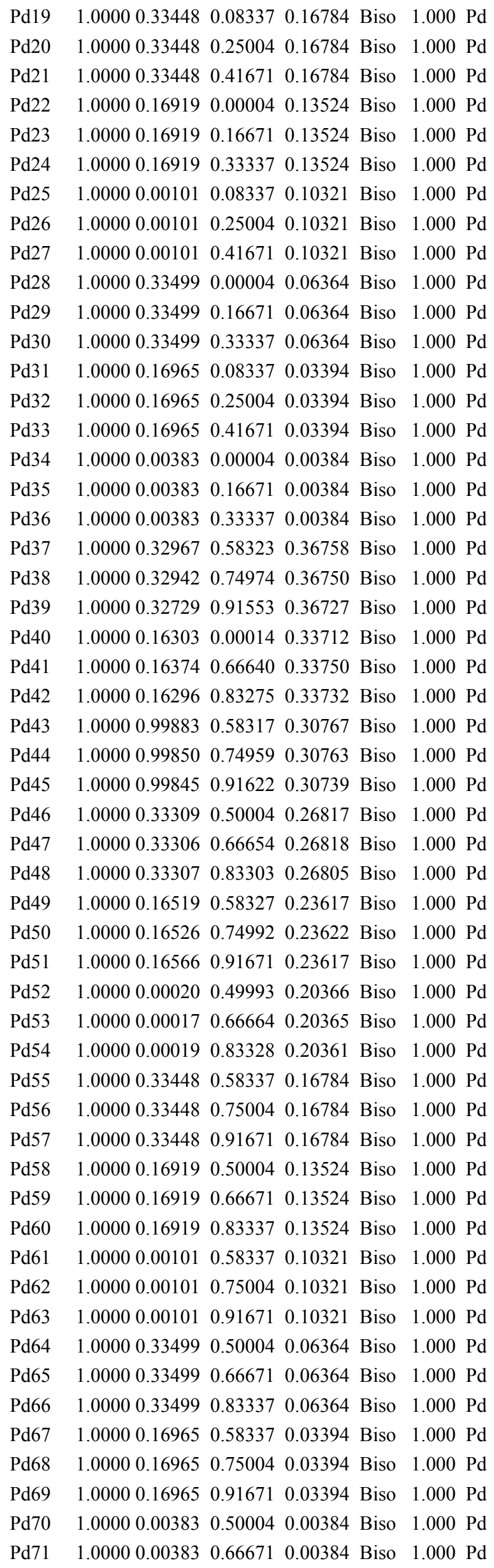




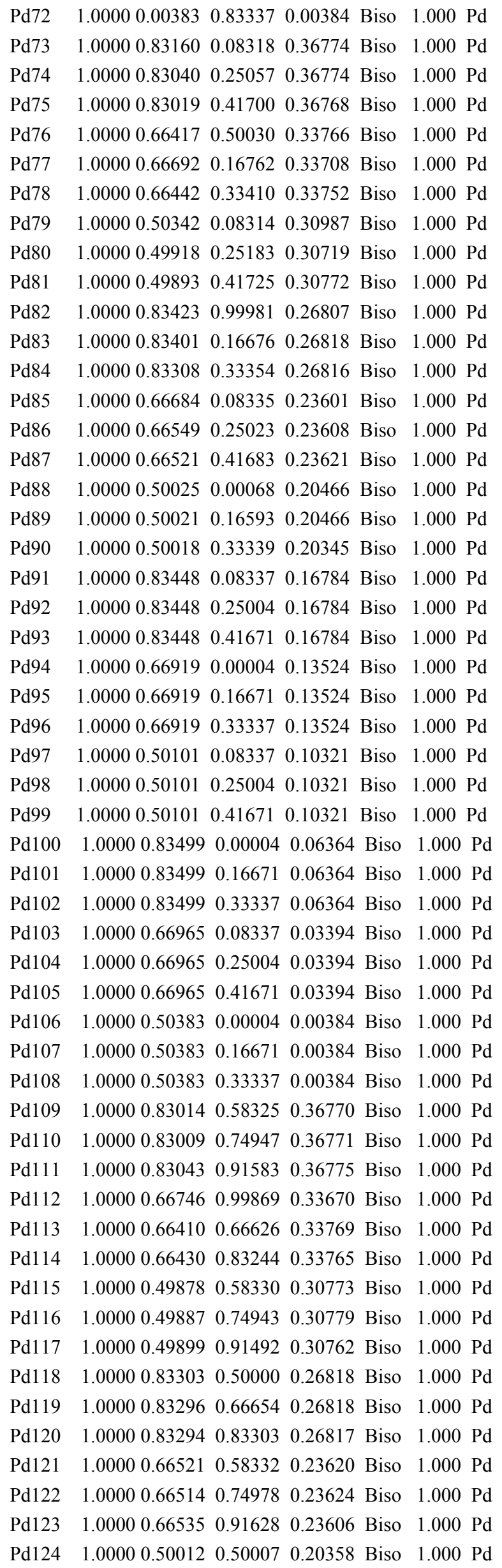




\begin{tabular}{|c|c|c|c|c|}
\hline $\operatorname{Pd} 125$ & 1.00000 .50009 & 0.66666 & 0.20360 Biso & $1.000 \mathrm{Pd}$ \\
\hline $\operatorname{Pd} 126$ & 1.00000 .50011 & 0.83331 & 0.20351 Biso & $1.000 \mathrm{~F}$ \\
\hline $\operatorname{Pd} 127$ & 1.00000 .83448 & 0.58337 & 0.16784 Biso & $1.000 \mathrm{I}$ \\
\hline $\operatorname{Pd} 128$ & 1.00000 .83448 & 0.75004 & 0.16784 Biso & $1.000 \mathrm{I}$ \\
\hline $\operatorname{Pd} 129$ & 1.00000 .83448 & 0.91671 & 0.16784 Biso & $1.000 \mathrm{~F}$ \\
\hline $\operatorname{Pd} 130$ & 1.00000 .66919 & 0.50004 & 0.13524 Biso & $1.000 \mathrm{I}$ \\
\hline $\operatorname{Pd} 131$ & 1.00000 .66919 & 0.66671 & 0.13524 Biso & $1.000 \mathrm{P}$ \\
\hline $\operatorname{Pd} 132$ & 1.00000 .66919 & 0.83337 & 0.13524 Biso & $1.000 \mathrm{P}$ \\
\hline Pd133 & 1.00000 .50101 & 0.58337 & 0.10321 Biso & $1.000 \mathrm{P}$ \\
\hline Pd134 & 1.00000 .50101 & 0.75004 & 0.10321 Biso & $1.000 \mathrm{~F}$ \\
\hline $\operatorname{Pd} 135$ & 1.00000 .50101 & 0.91671 & 0.10321 Biso & $1.000 \mathrm{~F}$ \\
\hline Pd136 & 1.00000 .83499 & 0.50004 & 0.06364 Biso & $1.000 \mathrm{~F}$ \\
\hline Pd137 & 1.00000 .83499 & 0.66671 & 0.06364 Biso & $1.000 \mathrm{I}$ \\
\hline $\operatorname{Pd} 138$ & 1.00000 .83499 & 0.83337 & 0.06364 Biso & $1.000 \mathrm{~F}$ \\
\hline $\operatorname{Pd} 139$ & 1.00000 .66965 & 0.58337 & 0.03394 Biso & 1.000 \\
\hline Pd140 & 1.00000 .66965 & 0.75004 & 0.03394 Biso & $1.000 \mathrm{P}$ \\
\hline $\operatorname{Pd} 141$ & 1.00000 .66965 & 0.91671 & 0.03394 Biso & $1.000 \mathrm{~F}$ \\
\hline $\operatorname{Pd} 142$ & 1.00000 .50383 & 0.50004 & 0.00384 Biso & $1.000 \mathrm{P}$ \\
\hline $\operatorname{Pd} 143$ & 1.00000 .50383 & 0.66671 & 0.00384 Biso & 1.000 \\
\hline $\operatorname{Pd} 144$ & 1.00000 .50383 & 0.83337 & 0.00384 Biso & 1.000 \\
\hline $\mathrm{O} 1$ & 10000046760 & ר & 39717 & $1.000 \mathrm{O}$ \\
\hline H1 & 1.00000 .48557 & 0.03554 & 0.41216 Biso & $1.000 \mathrm{H}$ \\
\hline
\end{tabular}

\# Pd211(6x6).C1.P E $=-301.12 ; \mathrm{ZPVE}=\mathrm{eV}$; frequencies $=\mathrm{cm}^{\wedge}-1$

data image0

_cell_length_a 13.7854

_cell_length_b 16.8836

_cell_length_c 24

_cell_angle_alpha 90

_cell_angle_beta 90

_cell_angle_gamma 90

_symmetry_space_group_name_H-M "P 1"

_symmetry_int_tables_number 1

loop

_symmetry_equiv_pos_as_xyz

'x, y, z'

loop_

_atom_site_label

_atom_site_occupancy

_atom_site_fract_x

_atom_site_fract_y

_atom_site_fract_z

_atom_site_thermal_displace_type

_atom_site_B_iso_or_equiv

_atom_site_type_symbol

Pd1 $1.00000 .321320 .08251 \quad 0.37160$ Biso $1.000 \quad \mathrm{Pd}$

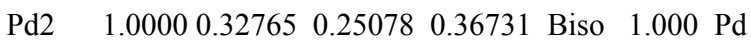

$\begin{array}{llllllll}\mathrm{Pd} 3 & 1.0000 & 0.32951 & 0.41687 & 0.36747 & \text { Biso } & 1.000 & \mathrm{Pd}\end{array}$ 


\begin{tabular}{|c|c|c|c|c|c|}
\hline $\operatorname{Pd} 4$ & 1.00000 .16370 & 0.50001 & 0.33755 Biso & 1.000 & \\
\hline Pd5 & 1.00000 .16326 & 0.16596 & 0.33725 Biso & 1.000 & \\
\hline Pd6 & 1.00000 .16316 & 0.33348 & 0.33737 Biso & 1.000 & \\
\hline 17 & 1.00000 .99913 & 0.08323 & 30756 Biso & 1.000 & \\
\hline 18 & 1.00000 .99846 & 0.25016 & 0.30750 Biso & 1.000 & \\
\hline 19 & 1.00000 .99860 & 0.41675 & 0.30771 Biso & 1.000 & $\mathrm{Pd}$ \\
\hline $\mathrm{d} 10$ & 1.00000 .33371 & 0.00019 & 0.26902 Biso & 1.000 & \\
\hline 111 & 1.0000 & & 26903 Biso & 1.000 & \\
\hline d12 & 1.00000 .3 & 0.3 & 804 Biso & 1.000 & d \\
\hline d13 & 1.00000 .16596 & 0.08327 & 615 Biso & 1.000 & \\
\hline d14 & 1.00000 .16549 & 0.24980 & 623 Biso & 1.000 & \\
\hline 115 & 1.00000 .16523 & 0.41661 & 26 Biso & 1.000 & \\
\hline 110 & 1.00000 .00057 & & 56 Biso & & \\
\hline 117 & 00000.00044 & 0.16663 & 0.20360 Biso & 1.000 & d \\
\hline 118 & 1.00000 .00018 & 0.33333 & 0.20362 Biso & 1.000 & d \\
\hline 119 & 1.00000 .33448 & 0.08337 & 784 Biso & 1.000 & d \\
\hline$d 20$ & 1.00000 .33448 & 0.25004 & Biso & 1.000 & $\mathrm{~d}$ \\
\hline $\operatorname{Pd} 21$ & 1.00000 .33448 & 0.41671 & 0.16784 Biso & 1.000 & $\mathrm{Pd}$ \\
\hline$d 22$ & 1.00000 & 0.00004 & 24 Biso & 1.00 & $\mathrm{Pd}$ \\
\hline 123 & 1.00000 .16919 & 0.16671 & 0.13524 Biso & 1.000 & $\mathrm{Pd}$ \\
\hline 124 & 1.00000 .16919 & 0.3 & 524 Biso & 1.000 & $\mathrm{Pd}$ \\
\hline 125 & 1.00000 .00101 & 0.08337 & 0.10321 Biso & 1.000 & $\mathrm{Pd}$ \\
\hline d26 & 1.00000 .00101 & 0.25004 & 0.10321 Biso & 1.000 & $\mathrm{Pd}$ \\
\hline $\operatorname{Pd} 27$ & 1.00000 .00101 & 0.41671 & 0.10321 Biso & 1.000 & $\mathrm{Pd}$ \\
\hline$d 28$ & 1.00000 .33499 & 0.00004 & 364 Biso & 1.000 & $\mathrm{Pd}$ \\
\hline $\operatorname{Pd} 29$ & 1.00000 .33499 & 0.16671 & 0.06364 Biso & 1.000 & $\mathrm{Pd}$ \\
\hline $\mathrm{d} 30$ & 1.00000 .33499 & 0.33337 & 0.06364 Biso & 1.000 & $\mathrm{Pd}$ \\
\hline 131 & 1.000 & 0.08337 & 0.03394 Biso & 1.000 & $\mathrm{Pd}$ \\
\hline 132 & 1.00000 .16965 & 0.25004 & 0.03394 Biso & 1.000 & $\mathrm{Pd}$ \\
\hline $\operatorname{Pd} 33$ & 1.00000 .16965 & 0.41671 & 0.03394 Biso & 1.000 & $\mathrm{Pd}$ \\
\hline $\operatorname{Pd} 34$ & 1.00000 .00383 & 0.00004 & 0.00384 Biso & 1.000 & $\mathrm{Pd}$ \\
\hline $\operatorname{Pd} 35$ & 1.00000 .00383 & 0.16671 & 0.00384 Biso & 1.000 & $\mathrm{Pd}$ \\
\hline $\operatorname{Pd} 36$ & 1.00000 .00383 & 0.33337 & 0.00384 Biso & 1.000 & $\mathrm{Pd}$ \\
\hline $\operatorname{Pd} 37$ & 1.00000 .32966 & 0.58326 & 0.36757 Biso & 1.000 & $\mathrm{Pd}$ \\
\hline $\mathrm{d} 38$ & 1.00000 .32938 & 0.74973 & 0.36747 Biso & 1.000 & $\mathrm{Pd}$ \\
\hline $\mathrm{d} 39$ & 1.00000 .32719 & 0.91546 & 0.36727 Biso & 1.00 & $\mathrm{Pd}$ \\
\hline $\mathrm{Pd} 40$ & 1.00000 .16281 & 0.00005 & 0.33713 Biso & 1.000 & $\mathrm{Pd}$ \\
\hline 141 & 1.00000 .16365 & 0.66636 & 0.33753 Biso & 1.000 & $\mathrm{Pd}$ \\
\hline Pd42 & 1.00000 .16286 & 0.83269 & 0.33734 Biso & 1.000 & $\mathrm{Pd}$ \\
\hline d43 & 1.00000 .99875 & 0.58317 & 0.30762 Biso & 1.000 & $\mathrm{Pd}$ \\
\hline $\mathrm{Pd} 44$ & 1.00000 .99841 & 0.74953 & 0.30762 Biso & 1.000 & $\mathrm{Pd}$ \\
\hline$d 45$ & 1.00000 .99832 & 0.91616 & 0.30739 Biso & 1.000 & $\mathrm{Pd}$ \\
\hline Pd46 & 1.00000 .33299 & 0.50004 & 0.26817 Biso & 1.000 & $\mathrm{Pd}$ \\
\hline Pd47 & 1.00000 .33302 & 0.66653 & 0.26817 Biso & 1.000 & $\mathrm{Pd}$ \\
\hline Pd48 & 1.00000 .33299 & 0.83305 & 0.26804 Biso & 1.000 & $\mathrm{Pd}$ \\
\hline Pd49 & 1.00000 .16517 & 0.58328 & 0.23619 Biso & 1.000 & $\mathrm{Pd}$ \\
\hline $\operatorname{Pd} 50$ & 1.00000 .16519 & 0.74991 & 23 Biso & 1.000 & $\mathrm{Pd}$ \\
\hline $\operatorname{Pd} 51$ & 1.00000 .16560 & 0.91669 & 0.23618 Biso & 1.000 & $\mathrm{Pd}$ \\
\hline $\operatorname{Pd} 52$ & 1.00000 .00013 & 0.49992 & 0.20365 Biso & 1.000 & $\mathrm{Pd}$ \\
\hline Pd53 & 1.00000 .00012 & 0.66664 & 0.20363 Biso & 1.000 & $\mathrm{Pd}$ \\
\hline $\operatorname{Pd} 54$ & 1.00000 .00013 & 0.83326 & 0.20360 Biso & 1.000 & $\mathrm{Pd}$ \\
\hline $\operatorname{Pd} 55$ & 1.00000 .33448 & 0.58337 & 0.16784 Biso & 1.000 & \\
\hline $\mathrm{d} 56$ & 1.00000 .33448 & 0.75004 & 0.16784 Biso & 1.000 & \\
\hline
\end{tabular}




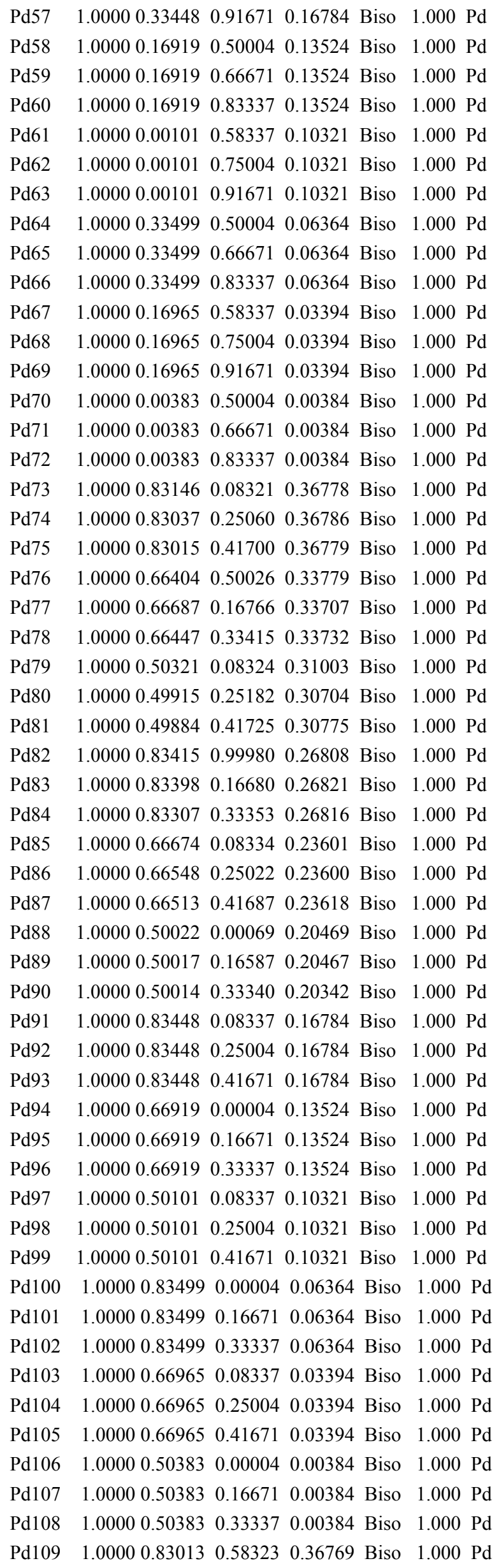




\begin{tabular}{|c|c|c|c|c|c|c|}
\hline 1110 & 1.00000 .83005 & & 40.36772 & Biso & & \\
\hline 1111 & 1.00000 .8303 & 0.91584 & $\begin{array}{ll}4 & 0.367\end{array}$ & Biso & 1.000 & \\
\hline 1112 & 1.00000 .66731 & 0.99873 & 33675 & Biso & .000 & \\
\hline & 1.00000 .66401 & 0.66623 & $\begin{array}{ll}3 & 0.33774\end{array}$ & Biso & 1.000 & \\
\hline d114 & 1.00000 .66422 & 0.83242 & 20.33769 & Biso & 1.000 & \\
\hline 1115 & 875 & & & & & \\
\hline d116 & 1.00000 & & $\begin{array}{ll}0 & 0.30778\end{array}$ & & & \\
\hline$d 117$ & 1.00000 & 0.91495 & & Biso & 1.000 & \\
\hline d118 & 1.00000 .83294 & 0.49999 & 90.26 & Biso & 000 & \\
\hline 1119 & 1.00000 .83290 & 0.66649 & & Biso & & \\
\hline & 1.00000 . & & & & & \\
\hline 1121 & 1.00000 & 0.58330 & & Biso & 1.000 & \\
\hline 1122 & 1.00000 & 0.7 & & Biso & & \\
\hline 1123 & 1.0000 & 0.9 & & Biso & & \\
\hline d124 & 1.00000 .50006 & 0.50007 & 70.20358 & Biso & 1.000 & \\
\hline d125 & 1.00000 .50008 & 0.66667 & $\begin{array}{ll}7 & 0.20\end{array}$ & Biso & 1.000 & \\
\hline$d 126$ & 1.00000. & 0.83 & & Biso & & \\
\hline 1127 & 1.0000 & 0.58 & & Biso & & \\
\hline 28 & 1.00 & 0.7 & & Biso & 1.000 & \\
\hline 1129 & 1.0000 & 0.91671 & & Biso & 1.000 & \\
\hline d130 & 1.00000 .66919 & 0.50004 & 40.13524 & Biso & 1.000 & \\
\hline$d 131$ & 1.0000 & 0.66671 & & Biso & 1.000 & \\
\hline$d 132$ & 1.00000 .6 & 0.83337 & & Biso & 1.000 & \\
\hline 1133 & 1.000 & 0.58337 & & Biso & 1.000 & $0 \mathrm{Pd}$ \\
\hline 1134 & 1.00 & 10.7 & & Biso & 1.000 & $0 \mathrm{Pd}$ \\
\hline 1135 & 1.0000 & 10.91 & & Biso & 1.000 & $0 \mathrm{Pd}$ \\
\hline$d 136$ & 1.00000 .83499 & 0.50004 & 40.06364 & 4 Biso & 1.000 & $0 \mathrm{P}$ \\
\hline d137 & 1.00000 .83499 & 0.66671 & 10.06364 & 4 Biso & 1.000 & $0 \mathrm{Pd}$ \\
\hline d138 & 1.00000 .83499 & 0.83337 & 70.06364 & 4 Biso & 1.000 & \\
\hline$d 139$ & 1.00000 .66965 & 0.58337 & & 4 Biso & 1.000 & \\
\hline$d 140$ & 1.0000 & 0.75004 & & 4 Biso & 1.000 & $0 \mathrm{P}$ \\
\hline d141 & 1.00 & 50.91671 & 10.0 & 4 Biso & 1.000 & 0 \\
\hline d142 & 1.0000 & 30.50004 & 40.00384 & 4 Biso & 1.000 & 0 \\
\hline$d 143$ & 1.00000 .50383 & 30.66671 & & 4 Biso & 1.000 & \\
\hline d144 & 1.00000 .50383 & 30.83337 & $\begin{array}{ll}7 & 0.00384\end{array}$ & 4 Biso & 1.000 & \\
\hline $\mathrm{O} 1$ & & & & & 1.00 & \\
\hline H1 & 1.00000 .48520 & 0.03472 & 1202 & Biso & $1.000 \mathrm{I}$ & \\
\hline $\mathrm{C} 1$ & 1.00000 .54568 & 0.250 & $0.48979 \mathrm{I}$ & Biso & 1.000 & \\
\hline $\mathrm{C} 2$ & 1.00000 .61370 & 0.32081 & $0.50030 \mathrm{I}$ & Biso & 1.000 & $\mathrm{C}$ \\
\hline $\mathrm{C} 3$ & 1.00000 .43922 & 0.27053 & $0.50142 \mathrm{I}$ & Biso & 1.000 & \\
\hline $\mathrm{H} 2$ & 1.00000 .56761 & 0.20045 & 0.51586 & Biso & $1.000 \mathrm{l}$ & \\
\hline H3 & 1.00000 .55312 & 0.23070 & 0.44655 & Biso & $1.000 \mathrm{I}$ & $\mathrm{H}$ \\
\hline H4 & 1.00000 .68931 & 0.30501 & 0.49190 & Biso & $1.000 \mathrm{l}$ & $\mathrm{H}$ \\
\hline H5 & 1.00000 .39221 & 0.21954 & 0.49337 & Biso & $1.000 \mathrm{I}$ & \\
\hline H6 & 1.00000 .59508 & 0.37111 & 0.47349 & Biso & $1.000 \mathrm{I}$ & \\
\hline $\mathrm{H} 7$ & 1.00000 .60913 & 0.34095 & 0.54359 & Biso & $1.000 \mathrm{I}$ & \\
\hline H8 & 1.00000 .42837 & 0.28850 & 0.54482 & Biso & $1.000 \mathrm{I}$ & \\
\hline H9 & 1.00000 .41467 & 0.31974 & 0.47508 & Biso & $1.000 \mathrm{I}$ & \\
\hline
\end{tabular}

\# Pd211(6x6).C1.1p E= -301.30 eV, ZPVE $=\mathrm{eV}$; frequencies $=\mathrm{cm}^{\wedge}-1$

data_image0 


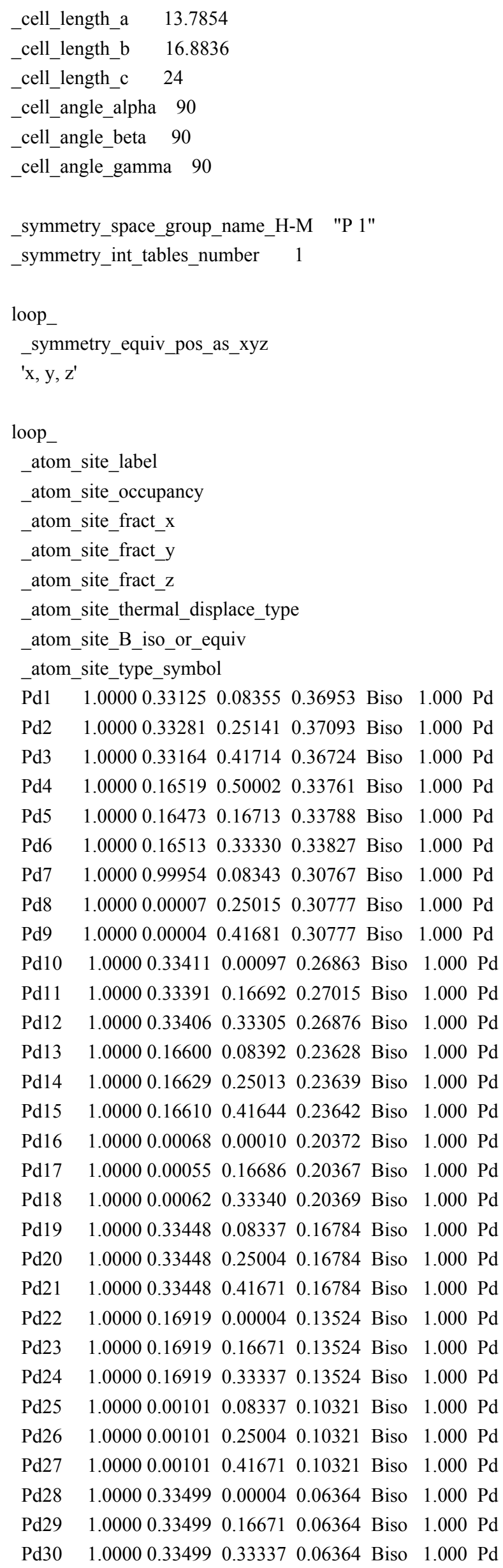


$\begin{array}{llllllll}\mathrm{Pd} 31 & 1.0000 & 0.16965 & 0.08337 & 0.03394 & \text { Biso } & 1.000 & \mathrm{Pd}\end{array}$

$\begin{array}{llllllll}\mathrm{Pd} 32 & 1.0000 & 0.16965 & 0.25004 & 0.03394 & \text { Biso } 1.000 \mathrm{Pd}\end{array}$

$\begin{array}{llllllll}\mathrm{Pd} 33 & 1.0000 & 0.16965 & 0.41671 & 0.03394 & \text { Biso } 1.000 \mathrm{Pd}\end{array}$

Pd34 1.00000 .003830 .000040 .00384 Biso 1.000 Pd

$\begin{array}{lllllllll}\mathrm{Pd} 35 & 1.0000 & 0.00383 & 0.16671 & 0.00384 & \text { Biso } 1.000 \mathrm{Pd}\end{array}$

$\begin{array}{llllllll}\mathrm{Pd} 36 & 1.0000 & 0.00383 & 0.33337 & 0.00384 & \text { Biso } 1.000 \mathrm{Pd}\end{array}$

$\begin{array}{llllllll}\mathrm{Pd} 37 & 1.0000 & 0.33083 & 0.58351 & 0.36751 & \text { Biso } 1.000 \mathrm{Pd}\end{array}$

$\begin{array}{llllllll}\mathrm{Pd} 38 & 1.0000 & 0.33051 & 0.75017 & 0.36754 & \text { Biso } 1.000 \mathrm{Pd}\end{array}$

$\begin{array}{llllllll}\mathrm{Pd} 39 & 1.0000 & 0.33052 & 0.91602 & 0.36696 & \text { Biso } 1.000 \mathrm{Pd}\end{array}$

$\begin{array}{lllllllll}\text { Pd40 } & 1.0000 & 0.16467 & 0.00019 & 0.33707 & \text { Biso } 1.000 & \text { Pd }\end{array}$

$\begin{array}{llllllll}\text { Pd41 } & 1.0000 & 0.16486 & 0.66667 & 0.33760 & \text { Biso } 1.000 & \text { Pd }\end{array}$

$\begin{array}{llllllll}\mathrm{Pd} 42 & 1.0000 & 0.16467 & 0.83338 & 0.33758 & \text { Biso } & 1.000 & \mathrm{Pd}\end{array}$

$\begin{array}{llllllll}\mathrm{Pd} 43 & 1.0000 & 0.99965 & 0.58345 & 0.30783 & \text { Biso } 1.000 \mathrm{Pd}\end{array}$

$\begin{array}{llllllll}\mathrm{Pd} 44 & 1.0000 & 0.99961 & 0.75000 & 0.30789 & \text { Biso } 1.000 \mathrm{Pd}\end{array}$

$\begin{array}{llllllll}\mathrm{Pd} 45 & 1.0000 & 0.99956 & 0.91666 & 0.30779 & \text { Biso } 1.000 \mathrm{Pd}\end{array}$

$\begin{array}{llllllll}\text { Pd46 } & 1.0000 & 0.33411 & 0.49993 & 0.26811 & \text { Biso } 1.000 & \text { Pd }\end{array}$

$\begin{array}{llllllll}\mathrm{Pd} 47 & 1.0000 & 0.33375 & 0.66669 & 0.26818 & \text { Biso } 1.000 \mathrm{Pd}\end{array}$

$\begin{array}{lllllll}\text { Pd48 } & 1.0000 & 0.33383 & 0.83343 & 0.26796 & \text { Biso } 1.000 & \mathrm{Pd}\end{array}$

$\begin{array}{llllllll}\text { Pd49 } & 1.0000 & 0.16578 & 0.58319 & 0.23635 & \text { Biso } 1.000 & \text { Pd }\end{array}$

$\begin{array}{llllllll}\text { Pd50 } & 1.0000 & 0.16573 & 0.75002 & 0.23628 & \text { Biso } 1.000 & \text { Pd }\end{array}$

$\begin{array}{lllllll}\mathrm{Pd} 51 & 1.0000 & 0.16612 & 0.91695 & 0.23620 & \text { Biso } 1.000 \mathrm{Pd}\end{array}$

Pd52 1.00000 .000620 .499990 .20371 Biso $1.000 \mathrm{Pd}$

$\begin{array}{llllllll}\mathrm{Pd} 53 & 1.0000 & 0.00047 & 0.66672 & 0.20372 & \text { Biso } 1.000 \mathrm{Pd}\end{array}$

$\begin{array}{lllllllll}\mathrm{Pd} 54 & 1.0000 & 0.00057 & 0.83338 & 0.20373 & \text { Biso } 1.000 \mathrm{Pd}\end{array}$

$\begin{array}{llllllll}\text { Pd55 } & 1.0000 & 0.33448 & 0.58337 & 0.16784 & \text { Biso } 1.000 & \text { Pd }\end{array}$

$\begin{array}{llllllll}\text { Pd56 } & 1.0000 & 0.33448 & 0.75004 & 0.16784 & \text { Biso } 1.000 & \mathrm{Pd}\end{array}$

$\begin{array}{llllllll}\mathrm{Pd} 57 & 1.0000 & 0.33448 & 0.91671 & 0.16784 & \text { Biso } 1.000 \mathrm{Pd}\end{array}$

$\begin{array}{lllllll}\mathrm{Pd} 58 & 1.0000 & 0.16919 & 0.50004 & 0.13524 & \text { Biso } 1.000 \mathrm{Pd}\end{array}$

$\begin{array}{llllllll}\text { Pd59 } & 1.0000 & 0.16919 & 0.66671 & 0.13524 & \text { Biso } 1.000 & \text { Pd }\end{array}$

$\begin{array}{lllllllll}\text { Pd60 } & 1.0000 & 0.16919 & 0.83337 & 0.13524 & \text { Biso } 1.000 & \text { Pd }\end{array}$

$\begin{array}{llllllllll}\text { Pd61 } & 1.0000 & 0.00101 & 0.58337 & 0.10321 & \text { Biso } 1.000 & \text { Pd }\end{array}$

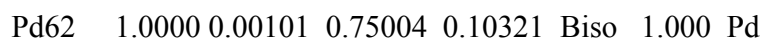

$\begin{array}{lllllllll}\text { Pd63 } 1.0000 & 0.00101 & 0.91671 & 0.10321 & \text { Biso } 1.000 & \mathrm{Pd}\end{array}$

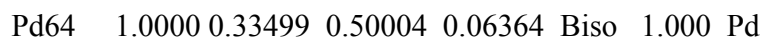

$\begin{array}{lllllllll}\text { Pd65 } & 1.0000 & 0.33499 & 0.66671 & 0.06364 & \text { Biso } 1.000 & \text { Pd }\end{array}$

$\begin{array}{lllllllll}\text { Pd66 } & 1.0000 & 0.33499 & 0.83337 & 0.06364 & \text { Biso } 1.000 & \text { Pd }\end{array}$

$\begin{array}{lllllllll}\text { Pd67 } & 1.0000 & 0.16965 & 0.58337 & 0.03394 & \text { Biso } 1.000 & \mathrm{Pd}\end{array}$

$\begin{array}{llllllll}\text { Pd68 } & 1.0000 & 0.16965 & 0.75004 & 0.03394 & \text { Biso } 1.000 & \text { Pd }\end{array}$

$\begin{array}{llllllll}\text { Pd69 } 1.0000 & 0.16965 & 0.91671 & 0.03394 & \text { Biso } 1.000 & \mathrm{Pd}\end{array}$

$\begin{array}{lllllll}\mathrm{Pd} 70 & 1.0000 & 0.00383 & 0.50004 & 0.00384 & \text { Biso } 1.000 \mathrm{Pd}\end{array}$

$\begin{array}{lllllllll}\mathrm{Pd} 71 & 1.0000 & 0.00383 & 0.66671 & 0.00384 & \text { Biso } 1.000 \mathrm{Pd}\end{array}$

$\begin{array}{llllllll}\mathrm{Pd} 72 & 1.0000 & 0.00383 & 0.83337 & 0.00384 & \text { Biso } 1.000 \mathrm{Pd}\end{array}$

$\begin{array}{llllllll}\mathrm{Pd} 73 & 1.0000 & 0.83084 & 0.08337 & 0.36772 & \text { Biso } 1.000 \mathrm{Pd}\end{array}$

$\begin{array}{llllllll}\mathrm{Pd} 74 & 1.0000 & 0.83100 & 0.25002 & 0.36774 & \text { Biso } 1.000 \mathrm{Pd}\end{array}$

$\begin{array}{llllllll}\text { Pd75 } & 1.0000 & 0.83122 & 0.41678 & 0.36772 & \text { Biso } 1.000 & \text { Pd }\end{array}$

$\begin{array}{llllllll}\mathrm{Pd} 76 & 1.0000 & 0.66533 & 0.50019 & 0.33771 & \text { Biso } 1.000 \mathrm{Pd}\end{array}$

Pd77 1.00000 .664950 .166690 .33760 Biso $1.000 \mathrm{Pd}$

$\begin{array}{llllllll}\operatorname{Pd} 78 & 1.0000 & 0.66544 & 0.33337 & 0.33762 & \text { Biso } 1.000 & \mathrm{Pd}\end{array}$

$\begin{array}{llllllll}\text { Pd79 } 1.0000 & 0.49988 & 0.08326 & 0.30776 & \text { Biso } 1.000 & \text { Pd }\end{array}$

$\begin{array}{llllllll}\mathrm{Pd} 80 & 1.0000 & 0.49992 & 0.25042 & 0.30794 & \text { Biso } 1.000 \mathrm{Pd}\end{array}$

$\begin{array}{lllllll}\mathrm{Pd} 81 & 1.0000 & 0.50075 & 0.41706 & 0.30753 & \text { Biso } 1.000 \mathrm{Pd}\end{array}$

$\begin{array}{llllllll}\mathrm{Pd} 82 & 1.0000 & 0.83378 & 0.00005 & 0.26826 & \text { Biso } & 1.000 & \mathrm{Pd}\end{array}$

$\begin{array}{llllllll}\mathrm{Pd} 83 & 1.0000 & 0.83380 & 0.16681 & 0.26829 & \text { Biso } & 1.000 & \mathrm{Pd}\end{array}$ 


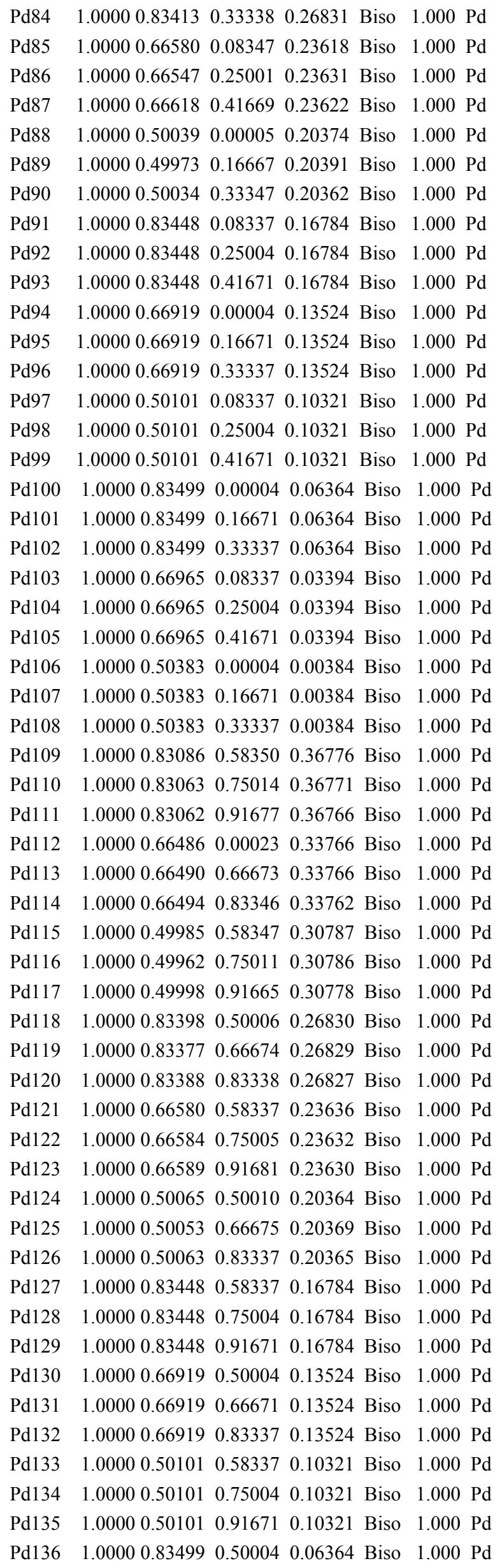




$\begin{array}{llllllll}\text { Pd137 } & 1.0000 & 0.83499 & 0.66671 & 0.06364 & \text { Biso } & 1.000 \mathrm{Pd} \\ \text { Pd138 } & 1.0000 & 0.83499 & 0.83337 & 0.06364 & \text { Biso } & 1.000 \mathrm{Pd} \\ \text { Pd139 } & 1.0000 & 0.66965 & 0.58337 & 0.03394 & \text { Biso } & 1.000 \mathrm{Pd} \\ \text { Pd140 } & 1.0000 & 0.66965 & 0.75004 & 0.03394 & \text { Biso } & 1.000 \mathrm{Pd} \\ \text { Pd141 } & 1.0000 & 0.66965 & 0.91671 & 0.03394 & \text { Biso } & 1.000 \mathrm{Pd} \\ \text { Pd142 } & 1.0000 & 0.50383 & 0.50004 & 0.00384 & \text { Biso } & 1.000 \mathrm{Pd} \\ \text { Pd143 } & 1.0000 & 0.50383 & 0.66671 & 0.00384 & \text { Biso } & 1.000 \mathrm{Pd} \\ \text { Pd144 } & 1.0000 & 0.50383 & 0.83337 & 0.00384 & \text { Biso } & 1.000 \mathrm{Pd} \\ \text { O1 } & 1.0000 & 0.27202 & 0.07617 & 0.46743 & \text { Biso } & 1.000 \mathrm{O} \\ \text { H1 } & 1.0000 & 0.26229 & 0.02007 & 0.47380 & \text { Biso } & 1.000 \mathrm{H} \\ \text { C1 } & 1.0000 & 0.46775 & 0.23553 & 0.46673 & \text { Biso } & 1.000 \mathrm{C} \\ \mathrm{C} 2 & 1.0000 & 0.49177 & 0.24569 & 0.52913 & \text { Biso } & 1.000 \mathrm{C} \\ \text { C3 } & 1.0000 & 0.36783 & 0.26766 & 0.45346 & \text { Biso } & 1.000 \mathrm{C} \\ \text { H2 } & 1.0000 & 0.47235 & 0.17267 & 0.45600 & \text { Biso } & 1.000 \mathrm{H} \\ \text { H3 } & 1.0000 & 0.52277 & 0.26636 & 0.44200 & \text { Biso } & 1.000 \mathrm{H} \\ \text { H4 } & 1.0000 & 0.56436 & 0.22269 & 0.53785 & \text { Biso } & 1.000 \mathrm{H} \\ \text { H5 } & 1.0000 & 0.20727 & 0.09705 & 0.46128 & \text { Biso } & 1.000 \mathrm{H} \\ \text { H6 } & 1.0000 & 0.49010 & 0.30815 & 0.54122 & \text { Biso } & 1.000 \mathrm{H} \\ \text { H7 } & 1.0000 & 0.43994 & 0.21362 & 0.55518 & \text { Biso } & 1.000 \mathrm{H} \\ \text { H8 } & 1.0000 & 0.30913 & 0.23622 & 0.47481 & \text { Biso } & 1.000 \mathrm{H} \\ \text { H9 } & 1.0000 & 0.36176 & 0.33151 & 0.46128 & \text { Biso } & 1.000 \mathrm{H}\end{array}$

